\title{
Azumaya-Algebren und Oktavenalgebren auf algebraischen Varietäten
}

\author{
Dissertation \\ zur Erlangung des mathematisch-naturwissenschaftlichen Doktorgrades \\ „Doctor rerum naturalium“ \\ der Georg-August-Universität Göttingen \\ im Promotionsprogramm Mathematik \\ der Georg-August University School of Science (GAUSS) \\ vorgelegt von \\ Kristin Stroth \\ aus Düsseldorf
}

Göttingen, 2013 


\section{Betreuungsausschuss:}

Prof. Dr. Ulrich Stuhler, Mathematisches Institut Prof. Dr. Thomas Schick, Mathematisches Institut

\section{Mitglieder der Prüfungskommission:}

Referent: Prof. Dr. Ulrich Stuhler, Mathematisches Institut Korreferent: Prof. Dr. Thomas Schick, Mathematisches Institut

Weitere Mitglieder der Prüfungskommission:

Prof. Dr. Thorsten Hohage, Institut für Num. und Angew. Mathematik Jun.-Prof. Dr. Andrea Krajina, Institut für Mathematische Stochastik Prof. Dr. Russell Luke, Institut für Num. und Angew. Mathematik Prof. Dr. Viktor Pidstrygach, Mathematisches Institut

Tag der mündlichen Prüfung: 23. Oktober 2013 


\section{Inhaltsverzeichnis}

Einleitung $\quad$ v

1 Azumaya-Algebren und Brauergruppen 1

1.1 Azumaya-Algebren über Körpern, Ringen und Schemata . . . . 1

1.1.1 Azumaya-Algebren über Körpern . . . . . . . . . . . . 1

1.1.2 Azumaya-Algebren über Ringen und Schemata . . . . . 3

1.2 Eigenschaften von Azumaya-Algebren . . . . . . . . . 5

1.2.1 Skalarerweiterung . . . . . . . . . . . 5

1.2.2 Homomorphismen von Azumaya-Algebren . . . . . . . . 6

1.2.3 Torsion in der Brauergruppe . . . . . . . . . . 7

1.2.4 Die relative Brauergruppe . . . . . . . . . . . . 7

1.3 Die kohomologische Brauergruppe . . . . . . . . . . 7

1.3.1 Verschränkte Produkte . . . . . . . . . . . . . 7

1.3.2 Kohomologische Brauergruppe eines Körpers . . . . . . 8

1.3.3 Kohomologische Brauergruppe eines Schemas . . . . . . 9

2 Konstruktion von Azumaya-Algebren 11

2.1 Zyklisch verschränkte Produkte . . . . . . . . . . . . . . 11

2.2 Normrestalgebren . . . . . . . . . . . . . . . . . . . . 13

2.2.1 Normrestalgebren über Körpern _. . . . . . . . . . . 13

2.2.2 Normrestalgebren über Ringen . . . . . . . . . . . . . . 14

2.2.3 Quaternionenalgebren ............... . 14

2.3 Ein Ausdehnungskriterium für Azumaya-Algebren . . . . . . . 15

2.3.1 Aus der Theorie der Maximalordnungen . . . . . . . 15

2.3.2 Verzweigungsabbildung und Charaktergruppe . . . . . . 19

2.3 .3 Aus der K-Theorie . . . . . . . . . . . . . . . . 22

2.3.4 Ausdehnung als Garbe von Quaternionenalgebren . . . . 26

2.4 Konstruktion mit dem Cyclic-Covering-Trick . . . . . . . . . . 29

2.4.1 Zyklische Algebren über Körpern und Bimoduln . . . . 30

2.4.2 Zyklische Algebren über Ringen . . . . . . . . . . . . . 33

2.4.3 Garben zyklischer Algebren . . . . . . . . . . . . . 37

2.4.4 Konkretes Beispiel: Doppelte Überlagerung . . . . . . . 42 
3 Kompositionsalgebren $\quad 51$

3.1 Nichtassoziative Algebren . . . . . . . . . . . . . . . . 52

3.1.1 Skalarerweiterung .............. 52

3.2 Quadratische Formen .................. 52

3.2.1 Skalarerweiterung ................ 54

3.2.2 Nichtausgeartete Formen in Charakteristik 2 . . . . . . 54

3.3 Kompositionsalgebren über Körpern und über Ringen . . . . . 55

3.3.1 Skalarerweiterung ................ 55

3.3.2 Lokalisierung . . . . . . . . . . . . . . . . . 56

3.3.3 Gute Reduktion . . . . . . . . . . . . . . . . . 57

3.3.4 Norm der Eins, quadratische Gleichung, Konjugation . . 58

3.3.5 Die Struktur von Kompositionsalgebren . . . . . . . . . 60

3.3.6 Divisions- und zerfallende Kompositionsalgebren . . . . 63

3.3.7 Zusammenhang von Norm und Multiplikation . . . . . . 65

3.3.8 Das Zentrum einer Kompositionsalgebra . . . . . . . . . 67

3.3.9 Kompositionsalgebren über speziellen Körpern . . . . . 67

3.4 Weitere Untersuchungen von Kompositionsalgebren . . . . . . . 68

3.4.1 Kompositionsalgebren über Produktringen . . . . . . . . 68

3.4.2 Unteralgebra zweier Elemente . . . . . . . . . . . 70

3.4.3 Operiert eine Quaternionenunteralgebra auf einer Oktavenalgebra? ............... . 70

4 Maximalordnungen in Kompositionsalgebren $\quad \mathbf{7 3}$

4.1 Gitter in quadratischen Räumen und in Kompositionsalgebren 73

4.2 Maximalordnungen . . . . . . . . . . . . . . . . . . 76

4.3 Maximalordnungen über Hauptidealringen . . . . . . . . . . . . 81

4.4 Maximalordnungen über diskreten Bewertungsringen . . . . . . 85

4.4.1 Divisionsalgebren über vollständigen Körpern . . . . . . 85

4.4.2 Zerfallende Algebren (über vollständigen Körpern) . . . 90

4.4.3 Algebren über beliebigen diskret bewerteten Körpern . . 90

4.5 Maximalordnungen über $\mathbf{Z}$ und über Krullringen . . . . . . . . 91

4.5.1 Lokalisieren von Maximalordnungen . . . . . . . . . . . 92

4.5.2 Ganze Gitter über Krullringen . . . . . . . . . . . . . . 93

4.5.3 Maximalordnungen über Krullringen . . . . . . . . . . 96

4.5.4 Lokale Konstruktion reflexiver Gitter über Krullringen . 98

5 Garben von Oktavenalgebren und von Maximalordnungen 101

5.1 Garben von Oktavenalgebren . . . . . . . . . . . . . . . . 101

5.2 Garben von Maximalordnungen . . . . . . . . . . . . . . 103

5.2.1 Vergleich von Maximalordnungen und Oktavenalgebren 108

5.3 Beispiel einer Garbe von Oktavenalgebren . . . . . . . . . . . 109

5.3.1 Definition einer Quaternionenalgebra . . . . . . . . 109

5.3.2 Verdopplung zur Oktavenalgebra . . . . . . . . . . 110

5.3 .3 Garbifizierung . . . . . . . . . . . . . . . . . 112 
A Kommutative Algebra $\quad \mathbf{1 2 1}$

A.1 Moduln und Algebren - Glossar . . . . . . . . . . . . . . . . 121

A.2 Nakayamas Lemma . . . . . . . . . . . . . . . . . . 122

A.3 Lokale Eigenschaften . . . . . . . . . . . . . . . . 123

A.4 Skalarerweiterung . . . . . . . . . . . . . . . . . 124

A.5 Rang eines projektiven Moduls . . . . . . . . . . . . 125

A.6 Zusammenhängende Ringe . . . . . . . . . . . . . . . . 126

A.7 Going-up, Going-down . . . . . . . . . . . . . . 127

A.8 Bewertungstheorie . . . . . . . . . . . . . . . . . . 128

A.8.1 Henselsches Lemma . . . . . . . . . . . . . . 128

A.8.2 Fortsetzungen von Bewertungen . . . . . . . . . . 129

A.8.3 Verallgemeinerte Reihendarstellungen . . . . . . . . 131

A.9 Krullringe . . . . . . . . . . . . . . . . . 133

B Garben und Schemata 135

B.1 Modulgarben und Schemata . . . . . . . . . . . . . 135

B.2 Garben von Ordnungen . . . . . . . . . . . . . . . 136

$\begin{array}{ll}\text { Literaturverzeichnis } & 139\end{array}$

$\begin{array}{ll}\text { Symbolverzeichnis } & 145\end{array}$

$\begin{array}{ll}\text { Index } & 149\end{array}$ 



\section{Einleitung}

Diese Arbeit handelt von Azumaya-Algebren und Oktavenalgebren auf algebraischen Varietäten. Im ersten Teil werden Azumaya-Algebren auf algebraischen Flächen behandelt. Im zweiten Teil werden Oktavenalgebren und allgemeiner auch Kompositionsalgebren über Körpern und Ringen untersucht, Maximalordnungen in Kompositionsalgebren betrachtet sowie Garben von Oktavenalgebren und Maximalordnungen in Kompositionsalgebren eingeführt.

Azumaya-Algebren sind spezielle nichtkommutative, zentrale Algebren. Ein Beispiel hierfür sind die vierdimensionalen Quaternionenalgebren. Oktavenalgebren sind spezielle nichtkommutative und nichtassoziative Algebren und können als achtdimensionale Verallgemeinerung der Quaternionen angesehen werden. Wir stellen die beiden Gebiete in dieser Einleitung getrennt vor.

\section{Azumaya-Algebren}

Ein offenes Problem in der nichtkommutativen algebraischen Geometrie ist die Klassifikation nichtkommutativer Flächen. Um diesem Ziel einen Schritt näher zu kommen, beschäftigt sich vor allem eine Gruppe um Artin, de Jong [AdJ], Chan, Ingalls und Kulkarni [CK03, CI05] damit, Maximalordnungen auf Flächen abstrakt zu klassifizieren. Außerdem werden auch Modulräume von Vektorbündeln oder getwisteten Garben über einer Maximalordnung, deren generische Faser eine Azumaya-Algebra ist, untersucht, etwa von Hoffmann und Stuhler [HS05], Lieblich (unter anderem in [Lie07]) und Chan und Kulkarni [CK11]. Hoffmann und Stuhler nutzen dabei einen Ansatz über geometrische Invariantentheorie und verwenden unter anderem Mumfords Methoden aus [MF82], Lieblich hingegen arbeitet weit abstrakter über Stacks. Auf diese Weise werden die verschiedensten Existenzaussagen bewiesen, es ist jedoch recht schwierig, Garben von Azumaya-Algebren konkret anzugeben. Hierzu hat Chan in [Cha05] eine Methode entwickelt, eine größere Klasse von Azumaya-Algebren oder allgemeiner von Maximalordnungen zu erzeugen. Er verwendet dazu den von ihm auf nichtkommutative Flächen verallgemeinerten sogenannten Cyclic-Covering-Trick. Mit Hilfe einer endlichen Überlagerung mit zyklischer Automorphismengruppe und eines invertierbaren Bimoduls wird eine Maximalordnung erzeugt, die genau über einer vorgegebenen Kurve verzweigt. Dieses 
und weitere Resultate sollen im ersten Teil dieser Arbeit genauer untersucht werden.

In Kapitel 1 stellen wir die grundlegenden Definitionen und Charakterisierungen von Azumaya-Algebren und Brauergruppen über Körpern, Ringen und Schemata bereit. Auch die kohomologische Beschreibung der Brauergruppe eines Körpers sowie die kohomologische Brauergruppe eines Schemas werden eingeführt.

In Kapitel 2 werden verschiedene Methoden vorgestellt und untersucht, wie man Azumaya-Algebren erzeugen kann. Bekannte Konzepte über Körpern sind die zyklisch verschränkten Produkte und die Normrest- oder Symbolalgebren, die sich auch allgemeiner über Ringen konstruieren lassen. Dieses wird in den Abschnitten 2.1 und 2.2 vorgeführt. In Abschnitt 2.3 leiten wir ein Ausdehnungskriterium für Azumaya-Algebren her. Wir untersuchen, wann oder wie weit sich eine über dem Funktionenkörper einer projektiven algebraischen Fläche gegebene Azumaya-Algebra auf die Fläche als Garbe von AzumayaAlgebren ausdehnen lässt. In Abschnitt 2.4 untersuchen wir Chans Methode, mit dem Cyclic-Covering-Trick Maximalordnungen auf einer algebraischen Fläche zu erzeugen.

\section{Oktavenalgebren}

Ausgangspunkt für den zweiten Teil dieser Arbeit war die Überlegung, ob sich die obigen Konzepte auch verallgemeinern lassen, wenn man die AzumayaAlgebra im generischen Punkt durch eine - nichtassoziative - Oktavenalgebra ersetzt. Als ersten Schritt in diese Richtung wird eine Idee vorgestellt, wie man Garben von Oktavenalgebren und Garben von Maximalordnungen in einer Oktavenalgebra definieren kann, und abschließend ein Beispiel über dem dreidimensionalen projektiven Raum konstruiert und untersucht.

Kompositionsalgebren sind endliche, treuprojektive Moduln über kommutativen Ringen mit einer nicht notwendig assoziativen Multiplikation, die zudem eine nichtausgeartete quadratische Form $N$ besitzen, die Komposition erlaubt. Letzteres bedeutet hier, dass $N(x y)=N(x) N(y)$ für alle Elemente $x, y$ der Kompositionsalgebra gilt. Oktavenalgebren sind Kompositionsalgebren von Dimension 8 über Körpern beziehungsweise von konstantem Rang 8 über Ringen.

Oktaven wurden zuerst unabhängig voneinander von John T. Graves (1843) und von Arthur Cayley (1845) betrachtet. So, wie die komplexen Zahlen $\mathbb{C}$ aus $\mathbb{R}$ durch Adjunktion eines Elementes $i$ mit Quadrat $i^{2}=-1$ entstehen und auch die Hamiltonschen Quaternionen $\mathbb{H}$ aus $\mathbb{C}$ durch Hinzunahme eines zweiten Elementes $j$ mit Quadrat -1 und der Relation $j i=-i j$ erzeugt werden, kann auch Cayleys Oktavenalgebra aus $\mathbb{H}$ und einem weiteren Element $v$ mit 
Quadrat -1 und der Relation

$$
(a+b v)(c+d v)=(a c-\bar{d} b)+(d a+b \bar{c}) v
$$

für $a, b, c, d \in \mathbb{H}$ multiplikativ erzeugt werden. Es bezeichne dabei $\bar{x}$ das Konjugierte eines Elementes $x \in \mathbb{H}$. Die sich daraus ergebende Multiplikation ist weder assoziativ noch kommutativ. So ist etwa $i(j v)=(j i) v=-(i j) v \neq(i j) v$. Als Vektorraum über den reellen Zahlen wird diese Oktavenalgebra von den acht Elementen $1, i, j, i j, v, i v, j v,(i j) v$ erzeugt. Die Multiplikation dieser Basiselemente ohne die Eins lässt sich anschaulich sehr schön in der Fano-Ebene wie in Abbildung 1 darstellen, siehe etwa Baez in [Bae02, 2.1]: Das Produkt zweier Elemente ist das dritte Element auf derselben Geraden, jedoch abgeändert um ein Minuszeichen, falls die Elemente nicht zyklisch in Pfeilrichtung angeordnet sind. Jedes der sieben Basiselemente $\neq 1$ hat Quadrat -1 .

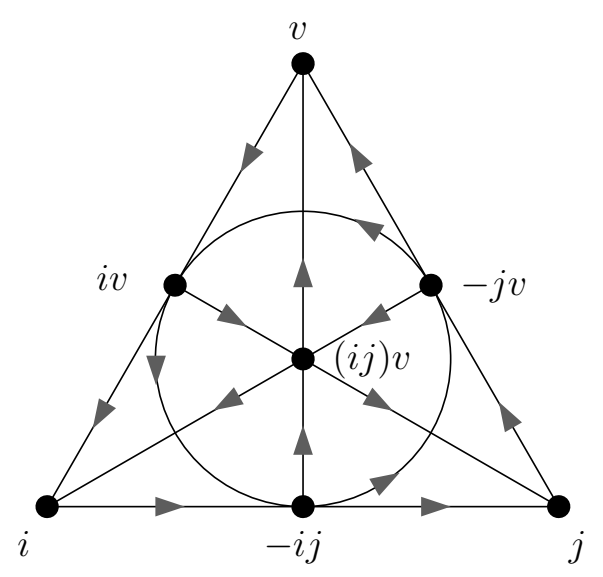

Abbildung 1: Fano-Ebene für die Erzeugenden der Cayley-Oktaven

Von Interesse sind die Oktavenalgebren unter anderem auch deshalb, weil ihre Automorphismengruppe eine zusammenhängende, einfache algebraische Gruppe vom Typ $G_{2}$ ist, siehe etwa Springer und Veldkamp [SV00, Thm. 2.3.5] für Körper beliebiger Charakteristik.

Anwendung finden die Oktaven daher auch in der aktuellen Forschung, etwa bei Gan und Yu [GY03], die die Oktaven im Zusammenhang mit der BruhatTits-Theorie für exzeptionelle algebraische Gruppen über lokalen Körpern untersuchen. Mit Hilfe der algebraischen Struktur der Oktaven wird die $\mathrm{G}_{2}$ charakterisiert. Auch bei den Untersuchungen von Roeseler [Roe11] kommt die unterliegende Algebrenstruktur der Oktaven bei der Untersuchung der $G_{2}$ und ihrer Reduktionstheorie zum Tragen.

Aber auch ohne konkrete Anwendungsbezüge werden Oktaven näher untersucht, so etwa beantwortet Gille in [Gil12] die von Petersson aufgeworfene Frage negativ, ob auch über beliebigen Ringen die Oktavenalgebra bereits eindeutig 
durch ihre Norm charakterisiert wird. Für Oktavenalgebren über lokalen Ringen und auch für Quaternionenalgebren über beliebigen Ringen ist dies hingegen zutreffend.

In Kapitel 3 dieser Arbeit werden Kompositionsalgebren über Ringen definiert und die grundlegenden Eigenschaften dargestellt. Die oben bereits angegebene Definition der Kompositionsalgebren wird dabei von Knus [Knu91, V (7.1)] übernommen. Sie findet sich so aber auch etwa bei Gille in [Gil12].

Die Eigenschaft, eine Kompositionsalgebra über einem Ring $R$ zu sein, ist lokal: Wir zeigen, dass wir über den lokalen Ringen $R_{\mathfrak{m}}$ der maximalen Ideale $\mathfrak{m} \subset R$ testen können, ob die lokalisierte Algebra eine Kompositionsalgebra ist, siehe Lemma 3.3.4.

Anders als bei Azumaya-Algebren muss hingegen die Quotientenalgebra einer Kompositionsalgebra modulo einem maximalen Ideal $\mathfrak{m}$ keine Kompositionsalgebra sein. In solch einem Fall sprechen wir von schlechter Reduktion modulo m, und diese wollen wir explizit erlauben.

In der Untersuchung der Eigenschaften von Kompositionsalgebren folgen wir im Wesentlichen den Ausführungen für Kompositionsalgebren über Körpern von Springer und Veldkamp in [SV00, Ch. 1] und untersuchen hier, wie wir die Aussagen für Algebren über Ringen verallgemeinern können.

Kompositionsalgebren über Körpern sind stets endlich-dimensional (auch wenn dies nicht explizit gefordert wird) und existieren nur in den Dimensionen 1, 2, 4 und 8. Ein Körper ist genau dann eine Kompositionsalgebra über sich selbst, wenn seine Charakteristik ungleich 2 ist. Jede Kompositionsalgebra von Dimension größer als 1 (bzw. in Charakteristik 2 von Dimension 4 oder 8) entsteht durch sogenannte Verdopplung aus einer Kompositionsalgebra halber Dimension, siehe etwa [SV00, Theorem 1.6.2]. Ähnliches gilt über Ringen für Kompositionsalgebren mit guter Reduktion und von konstantem Grad, siehe Abschnitt 3.3.5.

Auch über Integritätsbereichen gilt, dass wie über Körpern die Norm - also die geometrische Struktur - einer Kompositionsalgebra eindeutig durch ihre Algebrenstruktur bestimmt ist, siehe Korollar 3.3.24. Anders als über Körpern reicht umgekehrt über allgemeinen Ringen die geometrische Struktur jedoch nicht aus, um die algebraische festzulegen, wie Gille in [Gil12] zeigt, siehe auch Bemerkung 3.3.27.

Im vierten Abschnitt von Kapitel 3 folgen einige zusätzliche Untersuchungen: Wir zeigen in Unterabschnitt 3.4.1, dass das Produkt zweier Kompositionsalgebren eine Kompositionsalgebra über dem Produktring der beiden Grundringe ist, und konstruieren so eine Kompositionsalgebra, die weder konstanten Rang hat noch durch Verdopplung aus einer anderen Kompositionsalgebra entsteht. Außerdem überlegen wir in Unterabschnitt 3.4.3, ob und, wenn ja, wie eine Quaternionenunteralgebra auf einer Oktavenalgebra operieren kann. 
In Kapitel 4 betrachten wir Maximalordnungen in Kompositionsalgebren über Quotientenkörpern von Integritätsbereichen. Jede Kompositionsalgebra über einem Integritätsbereich erzeugt durch Skalarerweiterung eine Kompositionsalgebra über dem Quotientenkörper des Ringes. In dieser ist sie als erzeugendes Gitter enthalten. Wie im assoziativen Fall versucht man andersherum in einer gegebenen Kompositionsalgebra $C$ über dem Quotientenkörper nicht nur diejenigen Kompositionsalgebren über dem Ring zu bestimmen, die $C$ auf diese Art erzeugen, sondern betrachtet allgemeiner Ordnungen und speziell die Maximalordnungen in $C$.

Maximalordnungen in Kompositionsalgebren werden bereits in der klassischen Literatur untersucht. So betrachten van der Blij und Springer [vBS59] Maximalordnungen über vollständigen diskreten Bewertungsringen sowie über den ganzen Zahlen und den ganzen $p$-adischen Zahlen. Unter anderem zeigen sie in [vBS59, (3.4)], dass in der zerfallenden Oktavenalgebra über dem Quotientenkörper $K$ eines vollständigen diskreten Bewertungsringes $R$ alle Maximalordnungen über $R$ zueinander isomorph sind, siehe auch Satz 4.3.3. Dies verallgemeinert das assoziative Resultat, dass in der zerfallenden Quaternionenalgebra $\mathbb{M}_{2}(K)$ alle assoziativen Maximalordnungen durch Konjugation aus $\mathbb{M}_{2}(R)$ hervorgehen.

Dabei kennen van der Blij und Springer noch keine eigenständige Notation von Kompositionsalgebren über Ringen, sondern nutzen die Maximalordnungen als Definition ihrer sogenannten Oktavringe. Wir verallgemeinern in dieser Arbeit das Konzept von Maximalordnungen entsprechend der assoziativen Theorie auf beliebige Ringe und zeigen zum einen, dass jede Kompositionsalgebra $C$ über einem Integritätsbereich eine Ordnung in der durch Skalarerweiterung erzeugten Kompositionsalgebra $C_{K}$ über dem Quotientenkörper $K$ ist. Hat die Kompositionsalgebra $C$ gute Reduktion über allen Primidealen, so ist sie eine Maximalordnung in $C_{K}$, siehe Lemma 4.2.11. Zum anderen zeigen wir, dass umgekehrt über Hauptidealringen auch jede Ordnung eine Kompositionsalgebra ist - hier geht wiederum ein, dass wir bei den Kompositionsalgebren schlechte Reduktion erlauben -, siehe Lemma 4.3.1. Über beliebigen Ringen wird das im Allgemeinen nicht der Fall sein.

Eine Ordnung in einer Kompositionsalgebra $C$ über $K=\operatorname{Quot}(R)$ ist ein multiplikativ abgeschlossenes $R$-Gitter, das die Eins enthält und dessen Elemente ganz über $R$ sind, im Falle eines noetherschen Grundringes also eine endliche, nicht notwendig assoziative Algebra ganzer Elemente, die eine Basis von $C$ enthält. Ganz heißt hier, dass Norm und Spur der Elemente im Grundring liegen. Ist der Grundring ganzabgeschlossen, so ist ein multiplikativ abgeschlossenes Gitter automatisch ein ganzes Gitter. Ist der Grundring nicht ganzabgeschlossen, so fordern wir diese Eigenschaft von einer Ordnung zusätzlich. - Hiermit weichen wir von der klassischen Notation von Ordnungen in assoziativen Algebren ab. Sich auf ganze Gitter einzuschränken, hat jedoch den großen Vorteil, dass diese mit der Einschränkung der Norm selbst wieder 
quadratische Moduln sind. Letztlich bedeutet diese Abweichung keinen großen Unterschied, denn alle Ringe, die uns interessieren, werden ganzabgeschlossen und auch noethersch sein. Eine Maximalordnung ist eine Ordnung, die in keiner anderen Ordnung echt enthalten ist.

Über diskreten Bewertungsringen gelten auch für Maximalordnungen in Kompositionsalgebren ähnliche Resultate wie für ihre assoziativen Analoga in Azumaya-Algebren: So lässt sich im Falle einer Kompositionsdivisionsalgebra über einem vollständigen diskret bewerteten Körper die Bewertung eindeutig auf die Kompositionsalgebra fortsetzen, und deren Bewertungsring ist die eindeutige Maximalordnung, wie wir in den Sätzen 4.4.2 und 4.4.6 zeigen werden. Für Kompositionsalgebren über nicht vollständigen diskret bewerteten Körpern existiert wiederum eine Bijektion zwischen ihren Ordnungen und den Ordnungen in ihrer Vervollständigung, siehe Lemma 4.4.10.

Über den rationalen Zahlen gilt, dass ein Gitter in einer Oktavenalgebra genau dann eine Maximalordnung ist, wenn für jede Primzahl $p$ die Skalarerweiterung über die ganzen $p$-adischen Zahlen eine Maximalordnung ist, siehe etwa [vBS59, (4.1)]. Ist allgemeiner $R$ ein ganzabgeschlossener noetherscher Integritätsbereich, so zeigen wir in Satz 4.5.13 mit Hilfe der Sätze aus Bourbakis Kapitel über Divisoren [Bou72, VII], dass ganz analog ein $R$-Gitter in einer Kompositionsalgebra über dem Quotientenkörper genau dann eine Maximalordnung ist, wenn für jedes Primideal $\mathfrak{p}$ von Höhe 1 in $R$ die Skalarerweiterung bezüglich der Vervollständigung $\hat{R}_{\mathfrak{p}}$ eine Maximalordnung ist.

In Kapitel 5 setzen wir die zuvor betrachteten Konzepte von Oktavenalgebren und Maximalordnungen über Ringen auf normale, lokal-noethersche Schemata fort und führen Garben von Oktavenalgebren und Garben von Maximalordnungen ein. Auch diese können wiederum als nichtassoziative Verallgemeinerung von Maximalordnungen und Garben von Azumaya-Algebren verstanden werden.

Unter einer Garbe von Oktavenalgebren $(\mathcal{C}, N)$ verstehen wir eine kohärente Garbe $\mathcal{C}$ nichtassoziativer $\mathcal{O}_{X}$-Algebren und einen Morphismus $N: \mathcal{C} \rightarrow \mathcal{O}_{X}$, für die in jedem Punkt $x \in X$ die lokale Algebra $\left(\mathcal{C}_{x}, N_{x}\right)$ eine Oktavenalgebra über dem lokalen Ring $\mathcal{O}_{X, x}$ ist. Solch eine Garbe von Oktavenalgebren ist stets lokalfrei. Über einem affinen noetherschen Schema ist eine Garbe genau dann eine Oktavengarbe, wenn die Algebra der globalen Schnitte eine Oktavenalgebra ist. Wie auch schon bei den Kompositionsalgebren über Ringen erlauben wir dabei explizit schlechte Reduktion und fordern nicht, dass jede Quotientenalgebra $k(x) \otimes \mathcal{C}_{x}$ eine Oktavenalgebra über dem Restklassenkörper $k(x)=\mathcal{O}_{X, x} / \mathfrak{m}_{x}$ sein muss.

Es ist bereits im assoziativen Fall sinnvoll, neben den lokalfreien Azumaya-Algebren oder speziell in Dimension 4 den Quaternionenalgebren auch diejenigen torsionsfreien Garben zu betrachten, deren generische Faser eine vorgegebene 
Azumaya-Algebra über dem Funktionenkörper des Schemas ist. Diese Hinzunahme ergibt sich etwa in [HS05] als Kompaktifizierung der betrachteten Modulräume und liefert die Garben von Maximalordnungen in AzumayaAlgebren.

Unter einer nichtassoziativen Ordnung in einer Oktavenalgebra über dem Funktionenkörper eines ganzen, lokal-noetherschen Schemas verstehen wir in dieser Arbeit eine kohärente Garbe nichtassoziativer $\mathcal{O}_{X}$-Algebren, deren sämtliche Halme Ordnungen in der Oktavenalgebra sind. Ist $\mathcal{A}$ eine solche Ordnung und ist $U=\operatorname{Spec} R$ eine offene affine Teilmenge eines ganzabgeschlossenen Ringes $R$, so ist auch die Algebra $\mathcal{A}(U)$ eine Ordnung. Wir zeigen, dass auf einem normalen, lokal-noetherschen Schema eine Ordnung genau dann eine Maximalordnung ist, wenn sie in jedem Halm maximal ist, siehe Satz 5.2.7. Auch auf allen offenen affinen Mengen ist eine Maximalordnung maximal.

Jede Garbe von Oktavenalgebren ist dann eine Ordnung in der Algebra über dem generischen Punkt. Hat die Oktavengarbe gute Reduktion in jedem Punkt des Schemas, so ist sie eine Maximalordnung, siehe Lemma 5.2.9. Umgekehrt kann eine Maximalordnung allerdings schlechte Reduktion haben, wie wir in einem Beispiel zeigen werden. Auch muss eine Maximalordnung global nicht unbedingt eine Garbe von Oktavenalgebren sein. Jedoch ist in jedem Punkt von Kodimension 1 der Halm eine - wenn auch nicht zwangsläufig reguläre Oktavenalgebra.

Im letzten Abschnitt 5.3 des Kapitels 5 konstruieren wir auf dem dreidimensionalen projektiven Raum ganz explizit eine Garbe, die zugleich eine Garbe von Oktavenalgebren und eine Maximalordnung ist. Sie hat gute Reduktion auf einem offenen Unterschema, dessen Komplement Kodimension 1 hat, jedoch nicht auf dem gesamten Raum.

Im Anhang sind einige grundlegende Begriffe der kommutativen Algebra und der algebraischen Geometrie, einige der benutzten Konzepte der kommutativen Algebra, insbesondere aus der Bewertungstheorie, sowie die Definition von Garben von Ordnungen in Azumaya-Algebren aufgeführt.

Mit den Ergebnissen dieser Arbeit wird ein Grundstein für weitere Untersuchungen gelegt. Insbesondere die Definition von Garben von Oktavenalgebren und Maximalordnungen in Kapitel 5 und die zugehörigen ersten Resultate können dazu beitragen, nichtkommutative algebraische Räume zu klassifizieren.

Göttingen, den 5. September 2013

Kristin Stroth 


\section{Danksagung}

An dieser Stelle möchte ich mich ganz herzlich bei Herrn Prof. Dr. U. Stuhler für die Bereitstellung des interessanten Themas, die gute und fortwährende Betreuung, seine Geduld und die Ermutigung, diese Arbeit zu einem guten Ende zu führen, bedanken. Außerdem danke ich dem DFG-Graduiertenkolleg Gruppen und Geometrie sowie dem Mathematischen Institut für die Unterstützung. Vielen Dank auch an Ulrike Ober und vor allem an Karsten Roeseler für das Korrekturlesen und an Carsten Thiel für das LaTeX-Grundgerüst und eine wundervolle Zeit.

Kristin Stroth 


\section{Kapitel 1}

\section{Azumaya-Algebren und Brauergruppen}

In diesem Kapitel sind die Charakterisierungen von Azumaya-Algebren über Körpern, Ringen und Schemata sowie die Definition der Brauergruppe, einige elementare Eigenschaften und die kohomologische Beschreibung der Brauergruppe zusammengestellt.

Wenn nicht explizit angegeben, so sind die Beweise der angeführten Aussagen für Azumaya-Algebren über Körpern etwa bei Kersten [Ker90, Ker07] oder bei Gille und Szamuely [GS06], über lokalen Ringen und Schemata bei Milne [Mil80, Ch. IV] und über beliebigen Ringen in meiner Diplomarbeit [Str02, Kap. 2] oder etwa auch bei Knus [Knu91, Ch. III.5] oder Knus und Ojanguren [KO81], als Übungsaufgaben bei Bourbaki [Bou72, II.5 Exercises] und natürlich bei Auslander und Goldman [AG60a] zu finden.

Es bezeichne $K$ in diesem Kapitel einen Körper, $R$ einen kommutativen Ring mit Eins und $X$ ein lokal-noethersches Schema.

\subsection{Azumaya-Algebren über Körpern, Ringen und Schemata}

\subsubsection{Azumaya-Algebren über Körpern}

Eine Algebra über einem Körper heißt endlich-dimensional, wenn sie es als Vektorraum ist. Sie heißt zentral, wenn ihr Zentrum der Grundkörper ist, und sie heißt einfach, wenn sie als Ring keine zweiseitigen nichttrivialen Ideale besitzt.

Definition. Eine Algebra über einem Körper heißt Azumaya-Algebra, wenn sie endlich-dimensional, zentral und einfach ist. 
Teilweise werden Azumaya-Algebren über Körpern auch als zentral-einfache Algebren bezeichnet, insbesondere in der englischen Literatur wird in der Situation über Körpern zumeist von central simple algebras gesprochen.

Es bezeichne $A^{\mathrm{op}}$ die oppositionelle Algebra einer Algebra $A$, die sich aus dem $K$-Modul $A$ mit der gespiegelten Multiplikation $a \cdot b:=b a$ ergibt.

Satz 1.1.1. Sei A eine endlich-dimensionale K-Algebra. Dann sind äquivalent:

1. Es ist A eine Azumaya-Algebra über $K$.

2. Der kanonische K-Vektorraumhomomorphismus

$$
\begin{aligned}
\phi: A \otimes A^{\mathrm{op}} & \longrightarrow \operatorname{End}_{K}(A) \\
a \otimes b & \longmapsto(x \mapsto a x b)
\end{aligned}
$$

ist bijektiv.

3. Über dem algebraischen Abschluss $\bar{K}$ von $K$ gibt es eine $\bar{K}$-Algebrenisomorphie $A \otimes_{K} \bar{K} \cong \mathbb{M}_{n}(\bar{K})$ für ein $n \in \mathbb{N}$.

4. Es gibt eine endliche Körpererweiterung $L / K$ und eine L-Algebrenisomorphie $A \otimes_{K} L \cong \mathbb{M}_{n}(L)$ für ein $n \in \mathbb{N}$.

Es folgt, dass eine Azumaya-Algebra stets von quadratischer Dimension ist.

Da das Zentrum und die Ideale eines Matrizenringes $\mathbb{M}_{n}(A)$ einer Algebra $A$ nur von denen der Algebra $A$ abhängen, ist auch jeder Matrizenring über einer Azumaya-Algebra wieder eine Azumaya-Algebra.

Außerdem ist offensichtlich ist jeder zentrale, endlich-dimensionale Schiefkörper über $K$ eine Azumaya-Algebra. Wedderburn hat gezeigt, dass es im Wesentlichen keine weiteren gibt:

Satz 1.1.2 (Struktursatz von Wedderburn). Jede einfache, endlich-dimensionale $K$-Algebra A ist isomorph zu einem Matrizenring $\mathbb{M}_{n}(D)$ eines Schiefkörpers $D$ über $K$. Dabei ist D bis auf Isomorphie eindeutig bestimmt.

In der Darstellung $\mathbb{M}_{n}(A) \cong A \otimes_{K} \mathbb{M}_{n}(K)$ einer Azumaya-Algebra $A$ ergibt der zusätzliche zweite Faktor $\mathbb{M}_{n}(K)$ keine nennenswerten neuen Eigenschaften und es reicht, Azumaya-Algebren bis auf die sogenannte Ähnlichkeit zu untersuchen:

Definition. Zwei Azumaya-Algebren $A$ und $B$ über einem Körper $K$ heißen ähnlich, wenn eine Algebrenisomorphie $A \otimes_{K} \mathbb{M}_{n}(K) \cong B \otimes_{K} \mathbb{M}_{m}(K)$ für geeignete $n, m \in \mathbb{N}$ existiert. Wir schreiben dann $A \sim B$.

Ähnlichkeit ist eine Äquivalenzrelation auf der Menge der Azumaya-Algebren über einem festen Grundkörper. Die Ähnlichkeitsklasse, d. h. die Äquivalenzklasse unter der Ähnlichkeitsrelation einer Azumaya-Algebra $A$ sei mit $[A]$ bezeichnet. 
Das Tensorprodukt zweier Azumaya-Algebren ist ebenfalls eine AzumayaAlgebra. Außerdem sind für ähnliche Algebren $A \sim A^{\prime}$ bzw. $B \sim B^{\prime}$ auch die Produkte $A \otimes B$ und $A^{\prime} \otimes B^{\prime}$ ähnlich, denn es gilt $\mathbb{M}_{n}(K) \otimes \mathbb{M}_{m}(K) \cong \mathbb{M}_{n m}(K)$. Daher induziert das Tensorprodukt eine wohldefinierte Multiplikation auf der Menge der Ähnlichkeitsklassen von Azumaya-Algebren und wir erhalten:

Satz 1.1.3. Bezüglich der Operation $[A] \cdot[B]=[A \otimes B]$ bildet die Menge der Ähnlichkeitsklassen von Azumaya-Algebren über einem Körper $K$ eine abelsche Gruppe. Einselement ist die Klasse $[K]$ des Grundkörpers, Inverses zu $[A]$ ist die Klasse $\left[A^{\mathrm{op}}\right]$.

Definition. Diese Gruppe heißt die Brauergruppe $\operatorname{Br}(K)$ des Körpers $K$.

Der Struktursatz von Wedderburn ergibt, dass die Ähnlichkeitsklassen von Azumaya-Algebren und die Isomorphieklassen zentraler Schiefkörper in Bijektion zueinander stehen. Die Brauergruppe klassifiziert somit zugleich die zentralen Schiefkörpererweiterungen eines Körpers bis auf Isomorphie. Im Allgemeinen ist das Tensorprodukt zweier Schiefkörper jedoch kein Schiefkörper, daher ist es sinnvoll, alle Azumaya-Algebren zu betrachten.

\subsubsection{Azumaya-Algebren über Ringen und Schemata}

Eine Algebra $A$ über einem Ring $R$ ist ein nicht notwendig kommutativer Ring $A$ mit Eins zusammen mit einem Ringhomomorphismus $\phi: R \rightarrow A$, dessen Bild $\phi(R)$ im Zentrum $Z(A)$ von $A$ liegen muss: $\phi(R) \subseteq Z(A)$. Die Abbildung $\phi$ ist genau dann injektiv, wenn $A$ als $R$-Modul treu ist, d. h. wenn $\{r \in R \mid r a=0 \forall a \in A\}=0$ ist. Die Algebra heißt endlich, wenn sie als Modul endlich erzeugt ist. Sie heißt zentral, wenn ihr Zentrum gleich dem Bild des Grundringes ist: $\phi(R)=Z(A)$.

Die Verallgemeinerung von Azumaya-Algebren über Körpern auf solche über Ringen oder allgemeiner auf Garben von Azumaya-Algebren lautet dann:

Definition. Eine Algebra $A$ über einem Ring $R$ heißt Azumaya-Algebra, wenn sie endlich und treu ist und wenn für jedes maximale Ideal $\mathfrak{m} \subset R$ die Quotientenalgebra $A / \mathfrak{m} A$ eine Azumaya-Algebra über dem Restklassenkörper $R / \mathfrak{m}$ ist.

Definition. Eine Garbe $\mathcal{A}$ von $\mathcal{O}_{X}$-Algebren auf einem lokal-noetherschen Schema $\left(X, \mathcal{O}_{X}\right)$ heißt Garbe von Azumaya-Algebren (oder kurz auch nur Azumaya-Algebra), wenn sie als $\mathcal{O}_{X}$-Modul kohärent ist und wenn in jedem abgeschlossenen Punkt $x \in X$ der Halm $\mathcal{A}_{x}$ eine Azumaya-Algebra über dem lokalen Ring $\mathcal{O}_{X, x}$ ist.

Azumaya-Algebren über lokalen Ringen wurden zuerst von Azumaya [Azu51] betrachtet, über allgemeinen Ringen von Auslander und Goldman [AG60a] und über Schemata von Grothendieck [Gro68a]. 
Auslander und Goldman nutzen in ihrer Charakterisierung die Eigenschaft der Separabilität. Eine Algebra $A$ heißt separabel, wenn sie als $A \otimes A^{\mathrm{op}}-$ Modul projektiv ist.

Satz 1.1.4. Sei A eine endliche Algebra über einem Ring R. Dann sind äquivalent:

1. Es ist A eine Azumaya-Algebra über $R$.

2. Es ist A separabel, zentral und treu.

3. Für jedes maximale Ideal $\mathfrak{m} \subset R$ ist die Lokalisierung $A_{\mathfrak{m}}$ eine AzumayaAlgebra über dem lokalen Ring $R_{\mathfrak{m}}$.

4. Als R-Modul ist $A$ treu und projektiv und der kanonische $R$-Modulhomomorphismus

$$
\begin{aligned}
\phi: A \otimes A^{\mathrm{op}} & \longrightarrow \operatorname{End}_{R-\operatorname{Mod}}(A) \\
a \otimes b & \longmapsto(x \mapsto a x b)
\end{aligned}
$$

ist bijektiv.

5. Der kanonische Homomorphismus $\phi: A \otimes A^{\mathrm{op}} \rightarrow \operatorname{End}_{R}(A)$ ist bijektiv, und $A$ enthält $R$ als direkten Summanden.

Ist für eine Algebrengarbe über einem Schema die letzte Bedingung der Definition für alle abgeschlossenen Punkte erfüllt, so gilt sie auch für alle anderen Punkte. Außerdem ist jede Azumaya-Algebra $\mathcal{A}$ lokalfrei und von endlichem Rang als $\mathcal{O}_{X}$-Modul; der Rang muss allerdings nicht global konstant sein.

Satz 1.1.5 ([Mil80, Ch. IV, Proposition 2.1]). Sei $\mathcal{A}$ eine $\mathcal{O}_{X}$-Algebra, die als $\mathcal{O}_{X}$-Modul von endlichem Typ ist. Dann sind äquivalent:

1. Es ist $\mathcal{A}$ eine Azumaya-Algebra über $X$.

2. Als $\mathcal{O}_{X}$-Modul ist $\mathcal{A}$ lokalfrei und für jedes $x \in X$ ist $\mathcal{A}(x):=\mathcal{A}_{x} \otimes k(x)$ eine zentrale, einfache Algebra über dem Restklassenkörper $k(x)$.

3. Als $\mathcal{O}_{X}$-Modul ist $\mathcal{A}$ lokalfrei und der kanonische Homomorphismus $\mathcal{A} \otimes_{\mathcal{O}_{X}} \mathcal{A}^{\mathrm{op}} \rightarrow \operatorname{End}_{\mathcal{O}_{X}}(\mathcal{A})$ ist ein Isomorphismus.

4. Es gibt in der étalen Topologie eine Überdeckung $\left(U_{i} \rightarrow X\right)$ von $X$, für die es für jedes $i$ ein $n_{i} \in \mathbb{N}$ und eine Isomorphie $\mathcal{A} \otimes_{\mathcal{O}_{X}} \mathcal{O}_{U_{i}} \cong \mathbb{M}_{n_{i}}\left(\mathcal{O}_{U_{i}}\right)$ gibt.

5. Es gibt in der flachen Topologie eine Überdeckung $\left(U_{i} \rightarrow X\right)$ von $X$, für die es für jedes $i$ ein $n_{i} \in \mathbb{N}$ und eine Isomorphie $\mathcal{A} \otimes_{\mathcal{O}_{X}} \mathcal{O}_{U_{i}} \cong \mathbb{M}_{n_{i}}\left(\mathcal{O}_{U_{i}}\right)$ gibt. 
Die trivialen Beispiele für Azumaya-Algebren über Ringen sind die Endomorphismenringe von endlich erzeugten, treuen, projektiven $R$-Moduln und für Azumaya-Algebren über Schemata die Endomorphismengarben von lokalfreien $\mathcal{O}_{X}$-Moduln endlichen Ranges.

Auch über Ringen und Schemata werden Azumaya-Algebren modulo dieser trivialen Algebren betrachtet:

Definition. Zwei Azumaya-Algebren $A$ und $B$ über einem Ring $R$ heißen ähnlich, wenn es endlich erzeugte, treue, projektive $R$-Moduln $P_{1}$ und $P_{2}$ und eine Algebrenisomorphie $A \otimes_{R} \operatorname{End}_{R}\left(P_{1}\right) \cong B \otimes_{R} \operatorname{End}_{R}\left(P_{2}\right)$ gibt.

Zwei Garben von Azumaya-Algebren $\mathcal{A}$ und $\mathcal{B}$ auf dem Schema $\left(X, \mathcal{O}_{X}\right)$ heißen ähnlich, wenn es lokalfreie $\mathcal{O}_{X}$-Moduln $\mathcal{P}_{1}$ und $\mathcal{P}_{2}$ endlichen Ranges und eine Garbenisomorphie $\mathcal{A} \otimes_{\mathcal{O}_{X}} \underline{\operatorname{End}}_{\mathcal{O}_{X}}\left(\mathcal{P}_{1}\right) \cong \mathcal{B} \otimes_{\mathcal{O}_{X}} \underline{\operatorname{End}}_{\mathcal{O}_{X}}\left(\mathcal{P}_{2}\right)$ gibt.

Über einem lokalen Ring $R$ ist jeder endlich erzeugte, projektive Modul frei. Folglich ist sein Endomorphismenring nach Wahl einer Basis isomorph zu einer Matrixalgebra $\mathbb{M}_{n}(R)$. Die Ähnlichkeitsbedingung über lokalen Ringen sieht also aus wie über Körpern: Zwei Azumaya-Algebren $A$ und $B$ über einem lokalen Ring $R$ sind genau dann ähnlich, wenn $A \otimes_{R} \mathbb{M}_{n}(R) \cong B \otimes_{R} \mathbb{M}_{m}(R)$ für geeignete $m, n \in \mathbb{N}$ gilt.

Auch über Ringen und Schemata ist Ähnlichkeit eine Äquivalenzrelation auf der Menge der entsprechenden Azumaya-Algebren, und wie über Körpern erzeugt das Tensorprodukt eine wohldefinierte Multiplikation:

Satz 1.1.6. Bezüglich der Operation $[A] \cdot[B]=[A \otimes B]$ bildet die Menge der Ähnlichkeitsklassen von Azumaya-Algebren über einem Ring $R$ eine abelsche Gruppe. Einselement ist die Ähnlichkeitsklasse $[R]=\left[\operatorname{End}_{R}(P)\right]$ für jeden endlich erzeugten, treuen, projektiven $R$-Modul P. Inverses $z u[A]$ ist $\left[A^{\mathrm{op}}\right]$.

Ebenso bildet die Menge der Ähnlichkeitsklassen von Azumaya-Algebren über einem lokal-noetherschen Schema $X$ bezüglich der Operation $[\mathcal{A}] \cdot[\mathcal{B}]=[\mathcal{A} \otimes \mathcal{B}]$ eine abelsche Gruppe. Einselement ist $\left[\mathcal{O}_{X}\right]=\left[\right.$ End $\left._{\mathcal{O}_{X}}(\mathcal{P})\right]$ für jeden lokalfreien $\mathcal{O}_{X}$-Modul $\mathcal{P}$ endlichen Ranges. Inverses zu $[\mathcal{A}]$ ist $\left[\mathcal{A}^{\mathrm{op}}\right]$.

Definition. Diese Gruppen heißen die Brauergruppe $\operatorname{Br}(R)$ des Ringes $R$ beziehungsweise die Brauergruppe $\operatorname{Br}(X)$ des Schemas $X$.

\subsection{Eigenschaften von Azumaya-Algebren}

\subsubsection{Skalarerweiterung}

Satz 1.2.1. Seien $S$ eine kommutative und A eine beliebige $R$-Algebra.

1. Ist A eine Azumaya-Algebra über $R$, so ist $A \otimes_{R} S$ eine Azumaya-Algebra über $S$. 
2. Ist $S$ treuflach über $R$, so gilt auch die Umkehrung: Ist $A \otimes_{R} S$ eine Azumaya-Algebra über $S$, so ist A eine Azumaya-Algebra über $R$.

Die Basiserweiterung $[A] \mapsto\left[A \otimes_{R} S\right]$ ist mit der Ähnlichkeitsrelation verträglich, sodass wir einen Gruppenhomomorphismus $r_{S / R}: \operatorname{Br}(R) \rightarrow \operatorname{Br}(S)$ erhalten, die sogenannte Restriktion.

Darüber hinaus ist $\operatorname{Br}(\cdot)$ ein Funktor von der Kategorie der kommutativen Ringe in die Kategorie der abelschen Gruppen. Entsprechend ist für Schemata auch $\operatorname{Br}(\cdot)$ ein Funktor von der Kategorie der Schemata in die der abelschen Gruppen.

\subsubsection{Homomorphismen von Azumaya-Algebren}

Da der Kern eines Algebrenhomomorphismus ein zweiseitiges Ideal ist, ist über Körpern jeder Endomorphismus einer Azumaya-Algebra bijektiv. Es gilt sogar (siehe etwa [Ker07, Satz 4.2]):

Satz 1.2.2 (Skolem-Noether). Seien A eine Azumaya-Algebra und B eine einfache, endlich-dimensionale Algebra über einem Körper K. Dann gibt es zu je zwei K-Algebrenhomomorphismen $\varphi, \psi: B \rightarrow A$ eine Einheit $u \in A^{\times}$, für die $\varphi(x)=u \psi(x) u^{-1}$ für alle $x \in B$ gilt.

Insbesondere ist jeder Endomorphismus einer Azumaya-Algebra ein innerer Automorphismus.

Auch über Ringen und Garben gibt es nur bestimmte Arten von Automorphismen auf Azumaya-Algebren. Der verallgemeinerte Satz von Skolem-Noether lautet für lokale Ringe (siehe etwa [Mil80, Ch. IV, Proposition 1.4]):

Satz 1.2.3 (Skolem-Noether für lokale Ringe). Jeder Automorphismus einer Azumaya-Algebra über einem lokalen Ring ist ein innerer.

Über beliebigen Ringen gilt (siehe etwa [KO74, Corollaire III.5.4]):

Satz 1.2.4 (Skolem-Noether für Ringe). Jeder Endomorphismus einer Azumaya-Algebra über einem Ring ist ein Automorphismus.

Auch für die Garben von Azumaya-Algebren über Schemata gilt eine Variante dieses Satzes (siehe etwa [Mil80, Ch. IV, Proposition 2.3]):

Satz 1.2.5 (Skolem-Noether für Schemata). Sei $\phi: \mathcal{A} \rightarrow \mathcal{A}$ ein Automorphismus einer Garbe von Azumaya-Algebren auf einem Schema X. Dann gibt es eine solche offene Überdeckung $\left(U_{i}\right)_{i \in I}$ von $X$, dass auf jeder offenen Mengen $U_{i}$ die Einschränkung $\left.\phi\right|_{U_{i}}$ der innere Automorphismus zu einer Einheit $u_{i} \in \Gamma\left(U_{i}, \mathcal{A}\right)^{\times}$ist. 


\subsubsection{Torsion in der Brauergruppe}

Definition. Es bezeichnen $\operatorname{Br}_{n}(R)=\left\{[A] \in \operatorname{Br}(R) \mid[A]^{n}=1\right\}$ und analog $\operatorname{Br}_{n}(X)$ die $n$-Torsion der Brauergruppe eines Ringes $R$ beziehungsweise eines Schemas $X$.

Ist $A$ eine Azumaya-Algebra von konstantem Rang $n^{2}$ über einem Ring $R$, so gilt für ihre Ähnlichkeitsklasse in der Brauergruppe $[A]^{n}=1$. Als endliche Algebra lässt sich jede Azumaya-Algebra in ein endliches Produkt von Algebren von konstantem Rang zerlegen, folglich ist die Brauergruppe eines Ringes eine Torsionsgruppe, siehe etwa Knus und Ojanguren [KO74, Thm. IV.6.1 und Cor. IV.6.2].

Ebenso ist die Brauergruppe eines Schemas, das nur aus endlich vielen $\mathrm{Zu}$ sammenhangskomponenten besteht, oder eines quasikompakten Schemas eine Torsionsgruppe, siehe etwa [Mil80, Ch. IV, Proposition 2.7] oder [Gro68a, Corollaire 1.5].

\subsubsection{Die relative Brauergruppe}

Oftmals ist es sinnvoll, statt der gesamten Brauergruppe nur gewisse Untergruppen zu betrachten. Neben den Untergruppen $\mathrm{Br}_{n}$ der $n$-Torsion sind das insbesondere die sogenannten relativen Brauergruppen von Zerfällungskörpern:

Definition. Die relative Brauergruppe $\operatorname{Br}(L / K)$ einer Körpererweiterung $L / K$ ist der Kern der Restriktion $r: \operatorname{Br}(K) \rightarrow \operatorname{Br}(L),[A] \mapsto[A \otimes L]$.

Gehört $[A]$ zur relativen Brauergruppe $\operatorname{Br}(L / K)$, so gilt $A \otimes L \cong \mathbb{M}_{n}(L)$, und man sagt, $L$ zerfällt die Algebra $A$ bzw. $L$ ist ein Zerfällungskörper von $A$.

Nach Satz 1.1.1 besitzt jede Azumaya-Algebra über einem Körper $K$ einen Zerfällungskörper von endlichem Grad über $K$.

\subsection{Die kohomologische Brauergruppe}

\subsubsection{Verschränkte Produkte}

Definition. Eine Azumaya-Algebra $A$ über einem Körper $K$ heißt verschränktes Produkt, wenn sie einen über $K$ galoisschen Teilkörper $L$ mit $\left(\operatorname{dim}_{K} L\right)^{2}=$ $\operatorname{dim}_{K} A$ enthält.

Ist $A$ ein verschränktes Produkt mit passendem Teilkörper $L$, so liefert der Satz von Skolem-Noether zu jedem Automorphismus $\sigma \in \operatorname{Gal}(L / K)$, aufgefasst als Algebrenhomomorphismus $L \rightarrow A$, eine Einheit $u_{\sigma} \in A^{\times}$mit

$$
\sigma(x)=u_{\sigma} x u_{\sigma}^{-1}
$$


für alle $x \in L$. Zum einen sind dann die Elemente $u_{\sigma}$ linear unabhängig und es gilt $A=\bigoplus_{\sigma \in G} L u_{\sigma}$. Zum anderen definiert

$$
f(\sigma, \tau):=u_{\sigma} u_{\tau} u_{\sigma \tau}^{-1}
$$

einen 2-Kozykel aus $\mathcal{Z}^{2}\left(\operatorname{Gal}(L / K), L^{\times}\right)$und somit auch eine Kohomologieklasse $[f] \in \mathrm{H}^{2}\left(\operatorname{Gal}(L / K), L^{\times}\right)$.

Ist umgekehrt $L / K$ eine endliche Galoiserweiterung mit Galoisgruppe $G=$ $\operatorname{Gal}(L / K)$ gegeben, so liefert jedes Element der Kohomologiegruppe $\mathrm{H}^{2}\left(G, L^{\times}\right)$ ein verschränktes Produkt: Sei $f: G \times G \rightarrow L^{\times}$ein 2-Kozykel, dann definiert

$$
(L, G, f):=\bigoplus_{\sigma \in G} L u_{\sigma}
$$

mit den formalen Symbolen $u_{\sigma}$ und der durch

$$
x u_{\sigma} \cdot y u_{\tau}:=x \sigma(y) f(\sigma, \tau) u_{\sigma \tau}
$$

induzierten Multiplikation eine Azumaya-Algebra mit Zerfällungskörper $L$. Das Einselement dieser Algebra ist $f(1,1)^{-1} u_{1}$, entsprechend ist auch $L$ durch $x \mapsto x f(1,1)^{-1} u_{1}$ in $(L, G, f)$ eingebettet.

\subsubsection{Kohomologische Brauergruppe eines Körpers}

Jede Azumaya-Algebra $A$ über einem Körper $K$ ist ähnlich zu einem verschränkten Produkt $A^{\prime}$. Nach den obigen Überlegungen gilt $A^{\prime}=(L, G, f)$ mit einer geeigneten galoisschen Körpererweiterung $L / K$ mit Galoisgruppe $G$ und einem Kozykel $f \in \mathcal{Z}^{2}\left(G, L^{\times}\right)$.

Bei gegebener Erweiterung sind zwei verschränkte Produkte $(L, G, f)$ und $(L, G, h)$ genau dann ähnlich, wenn die Kozykel $g$ und $h$ kohomolog sind. Zusammen ergibt sich der folgende Satz:

Satz 1.3.1. Sei $L / K$ eine endliche galoissche Körpererweiterung. Dann gilt

$$
\operatorname{Br}(L / K) \cong \mathrm{H}^{2}\left(\operatorname{Gal}(L / K), L^{\times}\right) .
$$

Der algebraische Abschluss $\bar{K}$ von $K$ zerfällt jede Azumaya-Algebra über $K$, daher gilt insbesondere $\operatorname{Br}(K)=\operatorname{Br}(\bar{K} / K)$. Da jede Azumaya-Algebra zu einem verschränkten Produkt ähnlich ist, gilt $\operatorname{Br}(K)=\bigcup_{L / K} \operatorname{Br}(L / K)$, wobei die Vereinigung über alle endlichen Galoiserweiterungen $L / K$ laufe.

Ebenso erhält man die zweite Kohomologiegruppe $\mathrm{H}^{2}\left(\operatorname{Gal}(\bar{K} / K), \bar{K}^{\times}\right)$als induktiven Limes der Gruppen $\mathrm{H}^{2}\left(\operatorname{Gal}(L / K), L^{\times}\right)$. Da diese Konstruktion mit der Vereinigung der relativen Brauergruppen verträglich ist, ergibt sich der folgende Satz:

Satz 1.3.2. Sei $K$ ein Körper. Dann existiert eine natürliche Isomorphie zwischen der Brauergruppe und der zweiten Galoiskohomologiegruppe:

$$
\operatorname{Br}(K) \cong \mathrm{H}^{2}\left(\operatorname{Gal}(\bar{K} / K), \bar{K}^{\times}\right) .
$$




\subsubsection{Kohomologische Brauergruppe eines Schemas}

Auch die kohomologische Beschreibung der Brauergruppe lässt sich über Schemata verallgemeinern. Die richtige Kohomologie ist die étale.

Definition. Es heißt $\operatorname{Br}^{\prime}(X)=\mathrm{H}^{2}\left(X_{\mathrm{et}}, \mathbb{G}_{\mathrm{m}}\right)$ die kohomologische Brauergruppe eines Schemas $X$.

Da die Brauergruppe selbst eine Torsionsgruppe ist, wird gelegentlich als kohomologische Brauergruppe nur der Torsionsanteil $\mathrm{H}_{\text {tor }}^{2}$ betrachtet. Der Vergleich der beiden Gruppen $\operatorname{Br}(X)$ und $\operatorname{Br}^{\prime}(X)$ verhält sich insgesamt schwieriger als über Körpern.

Hauptsatz 1.3.3 ([Mil80, Ch. IV, Theorem 2.5]). Es gibt eine natürliche Injektion $i$ von der Brauergruppe $\operatorname{Br}(X)$ in die étale Brauergruppe $\operatorname{Br}^{\prime}(X)$.

Die Surjektivität ist im Allgemeinen eine offene Frage. Gabber zeigt, dass für ein beliebiges affines Schema der Torsionsanteil der kohomologischen Brauergruppe und die Brauergruppe übereinstimmen, siehe [Gab81, Ch. II, Thm. 1]. Milne zeigt, dass für das Spektrum eines henselschen Ringes oder für eine glatte Varietät über einem Körper Brauergruppe und kohomologische Brauergruppe übereinstimmen, siehe [Mil80, Ch. IV, Cor. 2.12 bzw. Prop. 2.15]. Allgemeiner haben inzwischen Gabber und de Jong ein Resultat für quasikompakte Schemata bewiesen:

Hauptsatz 1.3.4 ([dJ, 1.1 Theorem]). Sei $X$ ein quasikompaktes, separiertes Schema mit einer amplen invertierbaren Garbe. Dann stimmen Brauergruppe und kohomologische Brauergruppe überein: $\operatorname{Br}(X)=\operatorname{Br}^{\prime}(X)$.

Es gibt in der Tat Beispiele, für die die Brauergruppe und die kohomologische Brauergruppe nicht übereinstimmen. Etwa von Grothendieck eine normale, aber singuläre Fläche über den komplexen Zahlen.

Für beliebige quasikompakte Schemata sei noch das folgende Ergebnis von Milne zitiert:

Satz 1.3.5 ([Mil80, Ch. IV, Theorem 2.16]). Sei X ein quasikompaktes, lokalnoethersches Schema und sei $\gamma \in \operatorname{Br}^{\prime}(X)$. Dann existieren eine offene Teilmenge $U \subset X$ von $\operatorname{codim}(X-U)>1$ und eine Azumaya-Algebra $\mathcal{A}$ auf $U$ mit $i([\mathcal{A}])=\left.\gamma\right|_{U}$. Wenn $X$ regulär ist, kann $U$ von $\operatorname{codim}(X-U)>2$ gewählt werden.

Neben der kohomologischen Brauergruppe, die sich aus der étalen Topologie ergibt, wird gelegentlich auch die Zariski-Topologie herangezogen:

Definition. Es sei die Zariski-Brauergruppe $\operatorname{Br}\left(X_{\text {Zar }}\right)$ diejenige Untergruppe von $\operatorname{Br}(X)$, die von den Algebren erzeugt wird, die von einer ZariskiÜberdeckung von $X$ zerfällt werden. 
Letzteres heiße (analog zu Satz 1.1.5 (4)), dass es eine Zariski-Überdeckung $\left(U_{i}\right)$ und geeignete Isomorphismen $\mathcal{A} \otimes_{\mathcal{O}_{X}} \mathcal{O}_{U_{i}} \cong \mathbb{M}_{n_{i}}\left(\mathcal{O}_{U_{i}}\right)$ gebe. 


\section{Kapitel 2}

\section{Konstruktion von Azumaya-Algebren}

In diesem Kapitel werden verschiedene Konzepte, wie man Azumaya-Algebren ganz explizit erzeugen kann, vorgestellt und teilweise auch genauer untersucht.

In den beiden Abschnitten 2.1 und 2.2 betrachten wir die wohlbekannten zyklischen Algebren, einen Spezialfall der verschränkten Produkte, und die sogenannten Normrest- oder Symbolalgebren, eine andere Darstellungsmöglichkeit der zyklischen Algebren für den Fall, dass der Grundkörper genügend viele Einheitswurzeln enthält. Beide Arten von Algebren lassen sich ebenso über Ringen definieren und werden auch in den anderen beiden Abschnitten von Interesse sein.

In Abschnitt 2.3 geht es darum, eine über dem Funktionenkörper eines ganzen Schemas gegebene Azumaya-Algebra als Garbe von Azumaya-Algebren auf das Schema oder ein möglichst großes offenes Unterschema auszudehnen. Wir stellen verschiedene Kriterien vor, die testen, ob und wie weit eine Ausdehnung möglich ist. Speziell für Quaternionenalgebren über algebraischen Flächen konstruieren wir in Unterabschnitt 2.3.4 eine solche Ausdehnung.

In Abschnitt 2.4 untersuchen wir Chans Cyclic-Covering-Trick genannte Methode, eine große Klasse von Azumaya-Algebren mittels einer endlichen Überlagerung mit zyklischer Automorphismengruppe zu erzeugen (siehe [Cha05]).

\subsection{Zyklisch verschränkte Produkte}

Über einem Körper $K$ lassen sich Azumaya-Algebren recht einfach über eine Galoiserweiterung $L / K$ und einen 2-Kozykel $f$ der Galoiskohomologie als verschränkte Produkte $(L, \operatorname{Gal}(L / K), f)$ konstruieren, siehe Abschnitt 1.3.1.

Ein besonders übersichtlicher Spezialfall eines verschränkten Produktes ergibt sich, wenn die Galoisgruppe sogar zyklisch ist: 
Definition. Eine Azumaya-Algebra $A$ über einem Körper $K$ heißt zyklische Algebra oder zyklisch verschränktes Produkt, falls sie einen über $K$ zyklischen galoisschen Teilkörper $L$ mit $\left(\operatorname{dim}_{K} L\right)^{2}=\operatorname{dim}_{K} A$ enthält.

Sei $A$ eine zyklische Algebra von Dimension $n^{2}$ über $K$ mit passender zyklischer Galoiserweiterung $L / K$ von Grad $n$. Sei $\sigma$ ein erzeugendes Element der Galoisgruppe $G:=\operatorname{Gal}(L / K) \cong \mathbb{Z} / n \mathbb{Z}$. Ist $u=u_{\sigma} \in A^{\times}$eine Einheit mit $\sigma(x)=u_{\sigma} x u_{\sigma}^{-1}$ für alle $x \in L$, so erfüllt für jedes $i=0, \ldots, n-1$ auch $u_{\sigma^{i}}:=u^{i}$ die entsprechende Skolem-Noether-Bedingung $\sigma^{i}(x)=u^{i} x u^{-i}$ für alle $x \in L$. Der sich ergebende Kozykel $f$ hat dann die Form

$$
f\left(\sigma^{i}, \sigma^{j}\right)= \begin{cases}1, & \text { falls } i+j<n \\ u^{n}, & \text { falls } i+j \geq n\end{cases}
$$

für $0 \leq i, j<n$. Außerdem gilt $x=\sigma^{n}(x)=u^{n} x u^{-n}$ für alle $x \in L$ und offensichtlich auch $u^{n} u^{i}=u^{i} u^{n}$ für alle $i$. Mithin liegt $u^{n}$ im Zentrum der zentralen Algebra $\bigoplus_{\sigma \in G} L u_{\sigma}=A$, also gilt $u^{n} \in K^{\times}$.

Sei umgekehrt eine zyklische Galoiserweiterung $L / K$ von Grad $n$ mit erzeugendem Element $\sigma$ der Galoisgruppe $G$ gegeben. Dann definiert zu jedem $a \in K^{\times}$ die entsprechende Vorschrift

$$
f_{a}\left(\sigma^{i}, \sigma^{j}\right)=\left\{\begin{array}{ll}
1, & \text { falls } i+j<n \\
a, & \text { falls } i+j \geq n
\end{array} \quad \text { für } 0 \leq i, j<n\right)
$$

einen 2-Kozykel und es ist

$$
(L, \sigma, a):=\left(L, G, f_{a}\right)
$$

eine zyklische Azumaya-Algebra. Die obige Konstruktion dieses verschränkten Produktes ergibt die Zerlegung

$$
(L, \sigma, a)=\bigoplus_{i=0}^{n-1} L u^{i}
$$

mit einem formalen Symbol $u$ und mit den Multiplikationsvorschriften

$$
x u^{i} \cdot y u^{j}=x \sigma^{i}(y) u^{i+j} \quad \text { und } \quad u^{n}=a u^{0} .
$$

Sei $v \in L u, v \neq 0$. Dann gilt ebenfalls $v x=\sigma(x) v$ für alle $x \in L$. Ist $v=y u$ mit $y \in L$, so gilt $v^{n}=(y u)^{n}=y \sigma(y) \sigma^{2}(y) \ldots \sigma^{n-1}(y) u^{n}=\mathrm{N}_{L / K}(y) u^{n}$. Bei anderer Wahl des Erzeugers von $L u$ erhalten wir somit dieselbe Struktur bis auf die Norm eines Elementes.

Darüber hinaus gilt sogar, dass zwei zyklische Algebren $(L, \sigma, a)$ und $(L, \sigma, b)$ genau dann ähnlich - und somit auch isomorph - sind, wenn sich $a$ und $b$ nur um eine Norm unterscheiden, d.h. wenn es ein Element $z \in L^{\times}$mit $b=\mathrm{N}_{L / K}(z) a$ gibt, siehe etwa [Ker07, Satz 10.5]. Folglich ist bei gegebenen $L$ und $\sigma$ die Einheit $a=u^{n}$ eindeutig bestimmt als Element in $K^{\times} / \mathrm{N}_{L / K}\left(K^{\times}\right)$, der Gruppe der Normenreste. 


\subsection{Normrestalgebren}

Sei in diesem Abschnitt $n \in \mathbb{N}$ eine natürliche Zahl.

\subsubsection{Normrestalgebren über Körpern}

Sei $K$ ein Körper mit $\operatorname{char}(K) \nmid n$, der eine primitive $n$-te Einheitswurzel $\zeta$ enthalte. Beweise der Aussagen finden sich etwa bei Kersten [Ker07, Kap. 14].

Definition. Seien $a, b \in K^{\times}$. Dann ist die Normrestalgebra oder Symbolalgebra zu $a$ und $b$ definiert als $K$-Modul

$$
(a, b, \zeta):=\bigoplus_{i, j=0}^{n-1} K \cdot u^{i} v^{j}
$$

mit formalen Symbolen $u$ und $v$ zusammen mit der Multiplikation, die durch die Relationen

$$
u^{n}=a, \quad v^{n}=b, \quad v u=\zeta u v
$$

gegeben ist.

Die Normrestalgebra $(a, b, \zeta)$ ist eine Azumaya-Algebra von Dimension $n^{2}$ über $K$. Ihre Ähnlichkeitsklasse liegt in der $n$-Torsion der Brauergruppe: $[(a, b, \zeta)] \in \operatorname{Br}_{n}(K)$.

Auch als Element der Brauergruppe ist $[(a, b, \zeta)]$ abhängig von der Wahl der Einheitswurzel $\zeta$. Jede andere primitive Einheitswurzel in $K$ ist von der Form $\zeta^{r} \operatorname{mit} \operatorname{ggT}(r, n)=1$, für diese gilt $\left[\left(a, b, \zeta^{r}\right)\right]^{r}=[(a, b, \zeta)]$.

Von den weiteren Relationen, die gewisse Normrestalgebren zueinander erfüllen, seien hier diejenigen erwähnt, die für den später folgenden Zusammenhang zur K-Theorie wichtig sind:

Satz 2.2.1. Seien $a^{\prime}, a, b, c \in K^{\times}$mit $a^{\prime} \neq 1$. Dann gelten:

$$
\begin{aligned}
\left(a^{\prime}, 1-a^{\prime}, \zeta\right) & \sim K, \\
(a, b, \zeta) \otimes(c, b, \zeta) & \sim(a c, b, \zeta), \\
(a, b, \zeta) \otimes(a, c, \zeta) & \sim(a, b c, \zeta) .
\end{aligned}
$$

Jede Normrestalgebra ist ähnlich zu einer passenden zyklischen Algebra, die sich auch explizit angeben lässt:

Satz 2.2.2. Seien $a, b \in K^{\times}$zwei Einheiten. Setze $L=K(\sqrt[n]{b})$, dann gilt

$$
(a, b, \zeta) \sim(L, \sigma, a)
$$

für den Automorphismus $\sigma: \sqrt[n]{b} \mapsto \zeta^{-d} \sqrt[n]{b}$ von $L$ mit $d=[L: K]^{-1} n$. 


\subsubsection{Normrestalgebren über Ringen}

Sei $R$ ein Integritätsbereich mit $\operatorname{char}(R) \nmid n$, der eine primitive $n$-te Einheitswurzel $\zeta$ enthalte.

Wie über Körpern lässt sich dann mit den formalen Symbolen $u$ und $v$ der $R$-Modul

$$
(a, b, \zeta):=\bigoplus_{i, j=0}^{n-1} R \cdot u^{i} v^{j}
$$

definieren. Die Multiplikation, die sich entsprechend aus den Relationen

$$
u^{n}=a, \quad v^{n}=b, \quad v u=\zeta u v
$$

ergibt, definiert eine $R$-Algebrenstruktur auf $A$.

Die Algebra $(a, b, \zeta)$ muss im Allgemeinen keine Azumaya-Algebra über $R$ sein, jedoch gilt:

Satz 2.2.3. Seien $a, b \in R^{\times}$Einheiten. Ist $\mathfrak{m} \subset R$ ein maximales Ideal mit $\operatorname{char}(R / \mathfrak{m}) \nmid n$, so ist $(a, b, \zeta) \otimes_{R} R / \mathfrak{m}$ eine Azumaya-Algebra über $R / \mathfrak{m}$. Gilt $\operatorname{char}(R / \mathfrak{m}) \nmid n$ für jedes maximale Ideal $\mathfrak{m} \subset R$, so ist $(a, b, \zeta)$ eine AzumayaAlgebra über $R$.

BeweIs. Siehe etwa [Str02, Lemma 68/2.38].

Auch für Elemente $a, b \in R$, die nicht unbedingt Einheiten in $R^{\times}$sind, ergibt die obige Konstruktion eine endliche $R$-Algebra $(a, b, \zeta)$.

Die Elemente in dem vom Produkt uv erzeugten zweiseitigen Ideal in $(a, b, \zeta)$ sind von der Form

$$
\lambda \cdot a b+\left(\sum_{i>0}^{n-1} \alpha_{i} u^{i}+\sum_{j>0}^{n-1} \beta_{j} v^{j}+\sum_{i, j>0}^{n-1} \gamma_{i j} u^{i} v^{j}\right)
$$

mit geeigneten $\lambda, \alpha_{i}, \beta_{j}, \gamma_{i j} \in R$. Ist $a b$ tatsächlich keine Einheit in $R$, so gibt es ein maximales Ideal $\mathfrak{m} \subset R$ mit $a b \in \mathfrak{m}$ und damit mit $\overline{a b}=0$ in $R / \mathfrak{m}$. In der Algebra $(\bar{a}, \bar{b}, \bar{\zeta})$ über $R / \mathfrak{m}$ erzeugt die Restklasse $\overline{u v}$ damit ein nichttriviales Ideal: Dieses enthält nämlich keine Elemente aus $(R / \mathfrak{m}) \backslash\{0\}$, also insbesondere die 1 nicht. Daher sind weder die Quotientenalgebra $(\bar{a}, \bar{b}, \bar{\zeta})$ über $R / \mathfrak{m}$ noch die Algebra $(a, b, \zeta)$ über $R$ Azumaya-Algebren.

\subsubsection{Quaternionenalgebren}

Definition. Eine Azumaya-Algebra über einem Körper heißt Quaternionenalgebra, wenn sie vierdimensional ist. Eine Azumaya-Algebra über einem Ring heißt Quaternionenalgebra, wenn sie konstanten Rang 4 hat. 
Für $n=2$ und somit $\zeta=-1$ ergibt die obige Konstruktion der Normrestalgebren gerade die (über Körpern bekannte Darstellung der) Quaternionenalgebren. Ist der Zusammenhang klar, schreiben wir $(a, b)$ statt $(a, b,-1)$.

Über einem Körper $K$ von Charakteristik $\neq 2$ gilt auch umgekehrt, dass sich jede vierdimensionale Azumaya-Algebra als Normrestalgebra zu passenden Einheiten $a, b \in K^{\times}$darstellen lässt.

\subsection{Ein Ausdehnungskriterium für Azumaya-Algebren}

In diesem Abschnitt wollen wir ein Kriterium herleiten, wann oder wie weit sich eine gegebene Azumaya-Algebra $A$ über dem Funktionenkörper einer projektiven algebraischen Fläche als Garbe $\mathcal{A}$ von Azumaya-Algebren so auf die Fläche ausdehnen lässt, dass im generischen Punkt $\mathcal{A}_{\eta}=A$ gilt.

Im Wesentlichen handelt es sich bei diesem Abschnitt um eine Zusammenfassung der Ergebnisse meiner Diplomarbeit [Str02]. Dabei verallgemeinern wir in Unterabschnitt 2.3.4 die Situation von der projektiven Ebene auf beliebige reguläre, ganze, projektive Flächen.

Sei $\left(X, \mathcal{O}_{X}\right)$ eine lokal-noethersche, ganze, projektive algebraische Fläche.

Sind $\mathfrak{p}, \mathfrak{q} \in X$ Punkte mit $\mathfrak{q} \in \overline{\{\mathfrak{p}\}}$ und ist $\mathcal{A}$ eine Garbe auf einer offenen Umgebung $U$ von $\mathfrak{q}$, die in $\mathfrak{q}$ eine Azumaya-Algebra ist, so gilt erstens $\mathfrak{p} \in U$ und zweitens

$$
\mathcal{A}_{\mathfrak{p}}=\mathcal{A}_{\mathfrak{q}} \otimes_{\mathcal{O}_{\mathfrak{q}}} \mathcal{O}_{\mathfrak{p}}
$$

Folglich ist dann mit $\mathcal{A}_{\mathfrak{q}}$ auch $\mathcal{A}_{\mathfrak{p}}$ eine Azumaya-Algebra. Entscheidend ist also die Fortsetzbarkeit von $A$ in die Punkte $\mathfrak{p} \in X^{(1)}$ der Kodimension 1. Ist $\mathfrak{p} \in X^{(1)}$ ein solcher Punkt der Kodimension 1, so ist der lokale Ring $\mathcal{O}_{X, \mathfrak{p}}$ ein diskreter Bewertungsring.

Daher betrachten wir zunächst die Frage, wann sich eine gegebene AzumayaAlgebra $A$ über dem Quotientenkörper $K$ eines diskreten Bewertungsringes $R$ auf den Ring $R$ fortsetzen lässt, also wann eine Azumaya-Algebra $B$ über $R$ mit $B \otimes_{R} K=A$ existiert.

\subsubsection{Aus der Theorie der Maximalordnungen}

Sei $R$ ein noetherscher Integritätsbereich und $K=\operatorname{Quot}(R)$ sein Quotientenkörper.

\section{Gitter in Azumaya-Algebren}

Ein volles $R$-Gitter in einem endlich-dimensionalen $K$-Vektorraum $V$ ist ein endlich erzeugter $R$-Modul $M$, der $K \cdot M=V$ erfüllt. Offensichtlich liefert jede Vektorraumbasis von $V$ ein volles $R$-Gitter. 
Ist die Azumaya-Algebra $B$ über $R$ eine Fortsetzung der Azumaya-Algebra $A$ über $K$, so ist die Abbildung $B \rightarrow B \otimes_{R} K=A$ injektiv und $B$ kann als Unteralgebra von $A$ aufgefasst werden. Damit ist sie insbesondere ein volles $R$-Gitter in $A$.

Die Vervollständigung $\hat{R}$ eines diskreten Bewertungsringes $R$ ist ein treuflacher $R$-Modul (siehe etwa [Rei75, Corollary 2.22]). Daher entsprechen die vollen Gitter über einem diskreten Bewertungsring und die vollen Gitter über seiner Vervollständigung einander:

Satz 2.3.1 ([Rei75, nach Theorem 5.2]). Seien R ein diskreter Bewertungsring, $K=\operatorname{Quot}(R)$ sein Quotientenkörper und $\hat{R}$ und $\hat{K}$ ihre Vervollständigungen. Dann gilt:

1. Sei $M$ ein endlich erzeugter R-Modul. Dann ist die kanonische Abbildung $M \rightarrow M \otimes_{R} \hat{R}$ injektiv.

2. Sei $V$ ein endlich-dimensionaler $K$-Vektorraum und sei $\hat{V}:=V \otimes_{K} \hat{K}$. Dann definieren $M \mapsto M \otimes_{R} \hat{R}$ und $N \mapsto N \cap V$ eine inklusionserhaltende Bijektion und ihre Umkehrabbildung zwischen den vollen $R$-Gittern in $V$ und den vollen $\hat{R}$-Gittern in $\hat{V}$.

Diese Bijektion führt dazu, dass die Frage der Fortsetzbarkeit einer AzumayaAlgebra über einem diskret bewerteten Körper auf seinen Bewertungsring sich über den Vervollständigungen entscheiden lässt:

Satz 2.3.2. Seien $R$ ein diskreter Bewertungsring, $K=\operatorname{Quot}(R)$ sein Quotientenkörper und $\hat{R}$ und $\hat{K}$ ihre Vervollständigungen. Sei $A$ eine endliche $K$-Algebra und sei $\hat{A}:=A \otimes_{K} \hat{K}$.

Dann gibt es genau dann eine Azumaya-Algebra $B$ über $R$ mit $B \otimes_{R} K \cong A$, wenn es eine Azumaya-Algebra $B^{\prime}$ über $\hat{R}$ mit $B^{\prime} \otimes_{\hat{R}} \hat{K} \cong \hat{A}$ gibt.

BEweIs. Dies folgt unmittelbar aus dem letzten Satz und aus Satz 1.2.1. Für einen ausführlichen Beweis siehe [Str02, Satz 77/3.7].

\section{Maximalordnungen}

In Algebren werden vor allem solche vollen Gitter untersucht, die die Algebrenstruktur respektieren: Eine $R$-Ordnung in einer endlichen $K$-Algebra $A$ ist eine $R$-Unteralgebra von $A$, die als Modul ein volles $R$-Gitter in $A$ ist. Eine $R$-Ordnung in $A$ heißt maximale $R$-Ordnung in $A$ oder Maximalordnung, wenn sie in keiner anderen $R$-Ordnung echt enthalten ist.

Ist $L$ ein volles $R$-Gitter in der $K$-Algebra $A$, so definieren

$$
O_{\ell}(L)=\{x \in A \mid x L \subseteq L\} \quad \text { und } \quad O_{r}(L)=\{x \in A \mid L x \subseteq L\}
$$


Ordnungen in $A$, die sogenannte Links- bzw. Rechtsordnung von $L$ in $A$ (siehe etwa $[\operatorname{Rei} 75,(8.1)])$.

In den von uns betrachteten Fällen über noetherschen, ganzabgeschlossenen Integritätsbereichen werden stets Maximalordnungen existieren (siehe etwa [Rei75, Corollary 10.4]), im Allgemeinen ist das auch über noetherschen Ringen nicht der Fall (siehe etwa [Rei75, S. 110]).

Über vollständigen diskreten Bewertungsringen kann man die Maximalordnungen konkret bestimmen:

Satz 2.3.3 ([Rei75, Theorem 12.8]). Seien $R$ ein vollständiger diskreter Bewertungsring, $K=\operatorname{Quot}(R)$ sein Quotientenkörper und $D$ ein zentraler Schiefkörper über $K$. Dann existiert genau eine Maximalordnung in $D$, nämlich der ganze Abschluss von $R$ in $D$.

Dieser ganze Abschluss $\Delta$ lässt sich auch bewertungstheoretisch beschreiben: Ist $w: D^{\times} \rightarrow \mathbb{Q}$ die eindeutig existierende Fortsetzung der Bewertung von $K$, so gilt $\Delta=\{x \in D \mid w(x) \geq 0\}$. Die Maximalordnung ist also genau der Bewertungsring von $(D, w)$.

Die Maximalordnungen in einem Matrixring über einem Schiefkörper werden dann genau durch die Maximalordnung des Schiefkörpers in folgender Weise beschrieben:

Satz 2.3.4 ([Rei75, aus Theorem 17.3]). Seien $R$ ein vollständiger diskreter Bewertungsring, $K=\operatorname{Quot}(R)$ sein Quotientenkörper, D ein zentraler Schiefkörper über $K$ und $\Delta$ die eindeutige Maximalordnung in $D$.

Dann definiert $\Lambda=\mathbb{M}_{n}(\Delta)$ eine Maximalordnung in dem Matrixring $\mathbb{M}_{n}(D)$ über diesem Schiefkörper. Außerdem ist jede Maximalordnung in $\mathbb{M}_{n}(D)$ von der Form $u \Lambda u^{-1}$ mit einer Einheit $u \in \mathbb{M}_{n}(D)^{\times}=\mathrm{GL}_{n}(D)$, und umgekehrt ist auch jede Algebra dieser Form eine Maximalordnung in $\mathbb{M}_{n}(D)$.

Die Maximalordnung $u \Lambda u^{-1}$ ist gerade der Stabilisator von $u \Delta^{n}$ unter der Operation von $\mathbb{M}_{n}(D)=\operatorname{End}_{D}\left(D^{n}\right)$ auf $D^{n}$. Es gilt daher in kanonischer Weise $u \Lambda u^{-1}=\operatorname{End}_{\Delta}\left(u \Delta^{n}\right)$, und die unterschiedlichen Maximalordnungen in $\mathbb{M}_{n}(D)$ entsprechen den Einbettungen des Gitters $\Delta^{n}$ in $D^{n}$, die den Ursprung auf den Ursprung abbilden.

Wie wir oben gesehen haben, ist eine Azumaya-Algebra $B$ über $R$ ein volles $R$-Gitter in $A=B \otimes_{R} K$ und damit auch eine $R$-Ordnung in $A$. In speziellen Fällen ist sie sogar eine Maximalordnung:

Satz 2.3.5. Sei $R$ ein noetherscher, ganzabgeschlossener Integritätsbereich und $K$ sein Quotientenkörper. Ist B eine Azumaya-Algebra über $R$, so ist $B$ eine Maximalordnung in der Algebra $B \otimes_{R} K$.

BeweIs. Siehe [Str02, Satz 76/3.6] oder [AG60a, Proposition 7.1]. 
Hiermit und mit Hilfe der Eindeutigkeit der Maximalordnung in einem Schiefkörper lässt sich die Fortsetzbarkeit einer Azumaya-Algebra bereits in der Brauergruppe klären:

Satz 2.3.6. Sei $R$ ein diskreter Bewertungsring mit Quotientenkörper $K$. Seien $A$ eine Azumaya-Algebra über $K$ und $B^{\prime}$ eine Azumaya-Algebra über $R$, für die $\left[B^{\prime} \otimes_{R} K\right]=[A]$ in $\operatorname{Br}(K)$ gilt. Dann existiert eine Azumaya-Algebra $B$ über $R$ mit $B \otimes_{R} K \cong A$.

BEweis. Sei zunächst $R$ bzw. $K$ vollständig bezüglich der diskreten Bewertung. Nach dem Satz von Wedderburn gilt $A \cong \mathbb{M}_{r}(D)$ und $B^{\prime} \otimes_{R} K \cong \mathbb{M}_{n}(D)$ für einen zentralen Schiefkörper $D$ über $K$. Nach dem vorhergehenden Satz ist $B^{\prime}$ eine Maximalordnung in $\mathbb{M}_{n}(D)$ und nach Satz 2.3.4 somit von der Form $B^{\prime}=u \mathbb{M}_{n}(\Delta) u^{-1}$ mit einer Einheit $u \in \mathrm{GL}_{n}(D)$ und der eindeutigen Maximalordnung $\Delta$ in $D$. Mit $B^{\prime}$ ist auch die isomorphe Algebra $\mathbb{M}_{n}(\Delta)$ und damit auch $\Delta$ eine Azumaya-Algebra über $R$. Die $R$-Algebra $B:=\mathbb{M}_{r}(\Delta)$ ist dann eine gesuchte Fortsetzung von $A \cong \mathbb{M}_{r}(D)$.

Aus Satz 2.3.2 folgt damit die Aussage auch im Falle eines nicht vollständigen diskreten Bewertungsringes.

\section{Über regulären Ringen und Schemata}

Ein regulärer lokaler Ring ist ein noetherscher lokaler Ring $(R, \mathfrak{m})$, für den $\operatorname{dim} R=\operatorname{dim}_{R / \mathfrak{m}} \mathfrak{m} / \mathfrak{m}^{2}$ gilt, wobei $\operatorname{dim} R$ die Krull-Dimension des Ringes bezeichne. Ein regulärer Ring ist ein noetherscher Ring $R$, für den für jedes Primideal $\mathfrak{p} \subset R$ die Lokalisierung $R_{\mathfrak{p}}$ ein regulärer lokaler Ring ist.

Nach einem Theorem von Serre reicht es, die letzte Bedingung für alle maximalen Ideale von $R$ zu testen (siehe etwa [Mat86, Theorem 19.3 (Serre)]).

Außerdem ist jeder reguläre lokale Ring ein ganzabgeschlossener Integritätsbereich (siehe etwa [Mat86, Theorem 19.4]) und somit insbesondere ein Krullring. Die Lokalisierungen eines regulären Ringes in seinen Primidealen von Höhe 1 sind somit diskrete Bewertungsringe.

Satz 2.3.7 ([AG60a, Theorem 7.2]). Sei $R$ ein regulärer Integritätsbereich und $K=\operatorname{Quot}(R)$ sein Quotientenkörper. Dann ist die Restriktionsabbildung $\operatorname{Br}(R) \rightarrow \operatorname{Br}(K),[A] \mapsto\left[A \otimes_{R} K\right]$ injektiv.

BeweIs (Idee). Liegt $[B]$ im Kern der Restriktion, ist $B$ also eine AzumayaAlgebra über $R$ mit $B \otimes K=\mathbb{M}_{n}(K)$, so ist $B$ eine Maximalordnung in $\mathbb{M}_{n}(K)$, die zudem projektiv ist. Nach [AG60b, Thm.4.3] existiert dann ein endlich erzeugter, projektiver $R$-Modul $E$ mit $B=\operatorname{Hom}_{R}(E, E)$. Folglich ist auch $[B]$ trivial in der Brauergruppe. 
Folglich können wir die Brauergruppen des Ringes und seiner Lokalisierungen als Teilmenge der Brauergruppe des Quotientenkörpers auffassen:

$$
\operatorname{Br}(R) \subset \operatorname{Br}\left(R_{\mathfrak{p}}\right) \subset \operatorname{Br}(K) .
$$

Ebenso können unter den entsprechenden Voraussetzungen die Brauergruppe $\operatorname{Br}(X)$ eines Schemas $X$ und die Brauergruppen $\operatorname{Br}\left(\mathcal{O}_{X, x}\right)$ seiner lokalen Ringe als Teilmenge der Brauergruppe $\operatorname{Br}(k(X))$ des Funktionenkörpers betrachtet werden:

Ein Punkt $x \in X$ eines lokal-noetherschen Schemas $\left(X, \mathcal{O}_{X}\right)$ heißt regulär, wenn sein lokaler Ring $\mathcal{O}_{X, x}$ ein regulärer lokaler Ring ist. Das Schema selbst heißt regulär, wenn jeder Punkt $x \in X$ regulär ist.

Satz 2.3.8 ([Mil80, Ch. IV, Corollary 2.6]). Sei X ein reguläres, ganzes, lokalnoethersches Schema mit generischem Punkt $\eta$ und Funktionenkörper $k(X)$. Dann ist die kanonische Abbildung $\operatorname{Br}(X) \rightarrow \operatorname{Br}(k(X)),[\mathcal{A}] \mapsto\left[\mathcal{A}_{\eta}\right]$ injektiv.

Die Fortsetzbarkeit von Azumaya-Algebren auf reguläre Ringe oder reguläre Schemata lässt sich nun lokal entscheiden:

Satz 2.3.9 ([AG60a, Proposition 7.4], [Mil80, aus Ch. IV, Theorem 2.16]). Sei $R$ ein regulärer Integritätsbereich mit Quotientenkörper $K$, und sei $P$ die Menge seiner Primideale von Höhe 1. Dann gilt

$$
\operatorname{Br}(R)=\bigcap_{\mathfrak{p} \in P} \operatorname{Br}\left(R_{\mathfrak{p}}\right) .
$$

Sei zusätzlich $\operatorname{dim} R \leq 2$, dann gilt: Ist A eine Azumaya-Algebra über $K$, die $[A] \in \bigcap_{\mathfrak{p} \in P} \operatorname{Br}\left(R_{\mathfrak{p}}\right)$ erfüllt, so existiert eine Azumaya-Algebra $B$ über $R$ mit $B \otimes_{R} K \cong A$.

Lemma 2.3.10 ([Mil80, Ch. IV, Remark 2.18 (b), Beweis zu Theorem 2.16]). Sei $X$ ein reguläres, ganzes, lokal-noethersches Schema von Dimension 2 mit Funktionenkörper $k(X)$. Dann gilt

$$
\operatorname{Br}(X)=\bigcap_{x \in X^{(1)}} \operatorname{Br}\left(\mathcal{O}_{X, x}\right)
$$

Ist A eine Azumaya-Algebra über $k(X)$, die $[A] \in \bigcap_{x \in X^{(1)}} \operatorname{Br}\left(\mathcal{O}_{X, x}\right)$ erfüllt, so existiert eine Garbe $\mathcal{A}$ von Azumaya-Algebren auf $X$ mit $\mathcal{A}_{\eta} \cong A$.

\subsubsection{Verzweigungsabbildung und Charaktergruppe}

Definition. Sei $G=\operatorname{Gal}(L / K)$ die Galoisgruppe einer normalen Erweiterung $L / K$. Dann heißt

$$
X(G)=\mathrm{H}^{1}(G, \mathbb{Q} / \mathbb{Z})
$$

die Charaktergruppe von $G$. 
Die Körpererweiterung $L / K$ darf dabei auch von unendlichem Grad sein. Für die Charaktergruppe der absoluten Galoisgruppe $G_{K}=\operatorname{Gal}\left(K_{\mathrm{s}} / K\right)$ eines separablen Abschlusses $K_{\mathrm{s}}$ von $K$ schreiben wir auch $X(K)$ anstelle von $X\left(\operatorname{Gal}\left(K_{\mathrm{s}} / K\right)\right)$ oder von $X\left(G_{K}\right)$.

Da die Galoisgruppe trivial auf $\mathbb{Q} / \mathbb{Z}$ operiert, ist

$$
X(G)=\mathrm{H}^{1}(G, \mathbb{Q} / \mathbb{Z})=\mathcal{Z}^{1}(G, \mathbb{Q} / \mathbb{Z})=\operatorname{Hom}_{\text {cont }}(G, \mathbb{Q} / \mathbb{Z})
$$

gerade die Gruppe der stetigen Homomorphismen von $G$ nach $\mathbb{Q} / \mathbb{Z}$.

Außerdem gilt auch $X(G) \cong \mathrm{H}^{2}(G, \mathbb{Z})$, denn in der langen exakten Kohomologiesequenz der kurzen exakten Sequenz $0 \rightarrow \mathbb{Z} \rightarrow \mathbb{Q} \rightarrow \mathbb{Q} / \mathbb{Z} \rightarrow 0$ sind für $i>0$ die Gruppen $\mathrm{H}^{i}(G, \mathbb{Q})$ trivial, sodass der Verbindungshomomorphismus $\mathrm{H}^{1}(G, \mathbb{Q} / \mathbb{Z}) \rightarrow \mathrm{H}^{2}(G, \mathbb{Z})$ ein Isomorphismus ist.

Für vollständige diskrete Bewertungsringe beschreibt eine Charaktergruppe genau den Unterschied zwischen der Brauergruppe des Ringes und der seines Quotientenkörpers:

Satz 2.3.11. Sei $(R, \mathfrak{m})$ ein vollständiger diskreter Bewertungsring mit Quotientenkörper $K=\operatorname{Quot}(R)$ und Restklassenkörper $k=R / \mathfrak{m}$. Dann ist die Sequenz

$$
0 \rightarrow \operatorname{Br}(R) \stackrel{\text { res }}{\longrightarrow} \operatorname{Br}\left(K_{\mathrm{nr}} / K\right) \stackrel{r}{\longrightarrow} X(k) \rightarrow 0
$$

exakt und spaltet. Ist $k$ perfekt, so gilt $\operatorname{Br}\left(K_{\mathrm{nr}} / K\right)=\operatorname{Br}(K)$.

Dabei bezeichne $K_{\text {nr }}$ die maximal unverzweigte Erweiterung von $K$ in einem gewählten separablen Abschluss $K_{\mathrm{s}}$ von $K$.

Die Abbildung $r: \operatorname{Br}\left(K_{\mathrm{nr}} / K\right) \rightarrow X(k)$ heißt Verzweigungsabbildung: Zu jedem Element $[A] \in \operatorname{Br}\left(K_{\mathrm{nr}} / K\right)$ existiert ein endlicher, unverzweigter Zerfällungskörper $L / K$. Dessen unverzweigte Fortsetzung $v: L^{\times} \rightarrow \mathbb{Z}$ der Bewertung von $K$ induziert die Abbildung $v^{\times}: \mathrm{H}^{2}\left(\operatorname{Gal}(L / K), L^{\times}\right) \rightarrow \mathrm{H}^{2}(\operatorname{Gal}(L / K), \mathbb{Z})$, die zusammen mit den Isomorphismen $\operatorname{Br}(L / K) \cong \mathrm{H}^{2}\left(\operatorname{Gal}(L / K), L^{\times}\right)$und $\mathrm{H}^{2}(\operatorname{Gal}(L / K), \mathbb{Z}) \cong X(\operatorname{Gal}(L / K))$ die Abbildung $r$ ergibt.

Zum Beweis der Exaktheit und des Spaltens der Sequenz sei auf Serre [Ser79, Ch. XII, Thm. 2 und Ex.3] oder Grothendieck [Gro68b, Cor. (2.2)] verwiesen, beides folgt im Wesentlichen aus einer durch die Bewertung $v$ gegebenen Zerlegung $L^{\times}=\mathcal{O}_{L}^{\times} \times \mathbb{Z}$. Beide arbeiten statt mit der Brauergruppe $\operatorname{Br}(R)$ des Ringes mit der Brauergruppe $\operatorname{Br}(k)$ seines Restklassenkörpers. Für vollständige Bewertungsringe stimmen diese beiden Gruppen mittels der Restriktion $\operatorname{Br}(R) \rightarrow \operatorname{Br}(k),[A] \mapsto\left[A \otimes_{R} k\right]$ jedoch überein (siehe etwa [AG60a, Thm. 6.5]).

Ist $k$ nicht perfekt, so kann es Azumaya-Algebren über $K$ ohne unverzweigte Zerfällungskörper geben. Allerdings muss deren Ordnung durch $p$ teilbar sein: 
Lemma 2.3.12 ([Ser79, Ch. XII, Exercise 3]). Ist der Restklassenkörper $k$ nicht perfekt und hat Charakteristik $p$, so gehören alle Elemente $[A] \in \operatorname{Br}(K)$, deren Ordnung nicht durch $p$ teilbar ist, zur relativen Brauergruppe $\operatorname{Br}\left(K_{\mathrm{nr}} / K\right)$.

Da die Ordnung eines Elementes $[A]$ der Brauergruppe stets die Dimension der Algebra $A$ teilt, ist $\operatorname{char}(k) \nmid \operatorname{dim}_{K} A$ ein einfaches, aber nicht notwendiges Kriterium dafür, dass $[A]$ in $\operatorname{Br}\left(K_{\mathrm{nr}} / K\right)$ liegt.

Die Verzweigungsabbildung $r$ liefert dann ein Entscheidungskriterium für unsere Fortsetzungsfrage:

Satz 2.3.13. Sei $R$ ein diskreter Bewertungsring mit Quotientenkörper $K$ und Restklassenkörper $k$.

Dann gilt: Eine Azumaya-Algebra A über $K$ mit $\operatorname{char}(k) \nmid \operatorname{dim}_{K} A$ ist genau dann über $R$ fortsetzbar, wenn ihre Ähnlichkeitsklasse im Kern der Verzweigungsabbildung $r: \operatorname{Br}(K) \rightarrow X(k)$ liegt.

\section{Ausdehnung als Garbe von Azumaya-Algebren}

Sei nun $A$ eine Azumaya-Algebra über dem Funktionenkörper $K=k(X)$ einer regulären, ganzen algebraischen Fläche $X$. Sei $x \in X^{(1)}$ ein Punkt von Kodimension 1, für den $\operatorname{char}(k(x)) \nmid \operatorname{dim}_{K} A$ gelte. Dann ist $A$ nach dem letzten Satz genau dann über den lokalen $\operatorname{Ring} \mathcal{O}_{X, x}$ fortsetzbar, wenn $[A]$ im Kern der Verzweigungsabbildung $r_{x}: \operatorname{Br}(k(X)) \rightarrow X(k(x))$ liegt.

Gilt $r_{x}([A])=0$, so heißt $A$ unverzweigt in $x$. Andernfalls heißt $A$ verzweigt in $x$ und auch in der Kurve $\mathfrak{V}(x)$. In einem Punkt $y \in X$ beliebiger Kodimension heißt $A$ unverzweigt, wenn $y$ auf keiner Kurve $\mathfrak{V}(x)$ eines Punktes $x$ der Kodimension 1 liegt, in dem $A$ verzweigt. Andernfalls heißt $A$ verzweigt in $y$.

Hauptsatz 2.3.14. Sei A eine Azumaya-Algebra über dem Funktionenkörper $k(X)$ einer regulären, ganzen algebraischen Fläche $X$ über einem Körper $F$ und es gelte $\operatorname{char}(F) \nmid \operatorname{dim}_{k(X)} A$.

Dann gibt es eine Ausdehnung von A als Garbe von Azumaya-Algebren auf die offene Menge

$$
X \backslash \bigcup_{\substack{x \in X^{(1)} \\ r_{x}([A]) \neq 0}} \mathfrak{V}(x)
$$

und auf jede ihrer offenen Teilmengen. Ist umgekehrt $V$ eine offene Teilmenge, auf die eine Ausdehnung von A als Garbe von Azumaya-Algebren möglich ist, so ist $V$ eine Teilmenge der obigen offenen Menge.

Beweis. Dass $A$ nicht in die Punkte $x \in X^{(1)}$ mit $r_{x}([A]) \neq 0$ fortgesetzt werden kann, ist bereits klar. $\mathrm{Zu}$ zeigen bleibt, dass eine Ausdehnung von $A$ auf ein offenes Unterschema existiert, das alle Punkte $x \in X^{(1)}$ mit $r_{\mathfrak{p}}([A])=0$ enthält. 
Sei zunächst $X=\mathbb{A}_{F}^{2}$ die affine Ebene über $F$. Jede der Verzweigungsabbildungen $r_{\mathfrak{p}}: \operatorname{Br}(F(x, y)) \rightarrow X\left(k_{\mathfrak{p}}\right)$ für einen Punkt $\mathfrak{p} \in \mathbb{A}^{2}$ von Kodimension 1 ist eine Komposition der diskreten Bewertung $v_{\mathfrak{p}}$ mit weiteren Abbildungen. Da an jeder Stelle nur endlich viele Bewertungen ungleich null sind, gilt auch für die Verzweigungsabbildungen $r_{\mathfrak{p}}([A]) \neq 0$ nur für endlich viele $\mathfrak{p}$. Seien $f_{i} \in F[x, y]$ die Erzeuger dieser Primideale und sei $f$ ihr Produkt. Es sei $R=F[x, y]_{f}$. Dann enthält die offene Menge $D(f) \cong \operatorname{Spec}(R)$ von den Kodimension-1-Punkten $\mathfrak{p}$ genau diejenigen, in denen $A$ unverzweigt ist. Folglich existiert für jedes Primideal $\mathfrak{p} \subset R$ von Höhe 1 eine Azumaya-Algebra $A(\mathfrak{p})$ über $\mathcal{O}_{X, \mathfrak{p}}=R_{\mathfrak{p}}$, die $A$ fortsetzt. Nach Satz 2.3.9 existiert somit auch eine Azumaya-Algebra $B$ über $R$ mit $B \otimes_{R} R_{\mathfrak{p}} \cong A(\mathfrak{p})$ und vor allem mit $B \otimes_{R} F(x, y) \cong A$. Die zu $B$ assoziierte Garbe $\mathcal{A}$ ist dann eine Garbe von Azumaya-Algebren, die eine Ausdehnung von $A$ auf $\operatorname{Spec} R$ ist.

Ist $X$ nun eine beliebige reguläre Fläche, so können wir entsprechend die Algebra $A$ auf offene affine Teilmengen, auf denen $A$ unverzweigt ist, fortsetzen. Nach Lemma 2.3.10 existiert dann eine Garbe von Azumaya-Algebren auf deren Vereinigung.

\subsubsection{Aus der K-Theorie}

Quaternionenalgebren und allgemeiner auch Normrestalgebren (auch Symbolalgebren genannt) höherer Dimension lassen sich über Körpern mit hinreichend vielen Einheitswurzeln, also insbesondere über algebraisch abgeschlossenen Körpern der Charakteristik 0, auch mit Hilfe der algebraischen K-Theorie beschreiben. Zudem liefert das zahme Symbol ein einfaches Kriterium, ob eine Normrestalgebra im Kern einer Verzweigungsabbildung $r: \operatorname{Br}(K) \rightarrow X(k)$ liegt.

Wie jede abelsche Gruppe ist die Einheitengruppe $F^{\times}$eines Körpers $F$ in natürlicher Weise ein $\mathbb{Z}$-Modul: Es sei $n . a=a^{n}$ für $a \in F^{\times}$und $n \in \mathbb{Z}$.

Definition. Für einen Körper $F$ sind die nullte, erste und zweite algebraische K-Gruppe definiert als

$$
\begin{aligned}
& \mathrm{K}_{0}(F)=\mathbb{Z}, \\
& \mathrm{K}_{1}(F)=\left\{\{a\} \mid a \in F^{\times}\right\}, \\
& \mathrm{K}_{2}(F)=F^{\times} \otimes_{\mathbb{Z}} F^{\times} /\left\langle a \otimes(1-a) \mid a \in F^{\times}, a \neq 1\right\rangle .
\end{aligned}
$$

Die Restklasse von $a \otimes b \in F^{\times} \otimes_{\mathbb{Z}} F^{\times}$in $\mathrm{K}_{2}(F)$ werde mit $\{a, b\}$ bezeichnet. Die Verknüpfung der Gruppen wird additiv notiert.

Die zweite K-Gruppe lässt sich auch durch die Erzeugenden $\{a, b\}$ mit $a, b \in F^{\times}$ 
und die Relationen

$$
\begin{array}{ll}
\left\{a^{\prime}, 1-a^{\prime}\right\}=0 & \text { (Steinberg-Relation) } \\
\{a c, b\}=\{a, b\}+\{c, b\} & \text { (Bilinearität) } \\
\{a, b c\}=\{a, b\}+\{a, c\} &
\end{array}
$$

für alle $a^{\prime}, a, b, c \in F^{\times}, a^{\prime} \neq 1$ beschreiben.

Unmittelbar aus diesen Relationen ergeben sich die weiteren Identitäten $\{a,-a\}=0,\{a, a\}=\{a,-1\}$ und $\{a, b\}=-\{b, a\}$ für alle $a, b \in F^{\times}$.

Ist $L / F$ eine Körpererweiterung, so induziert die Einbettung $F \rightarrow L$ die beiden Gruppenhomomorphismen

$$
\begin{aligned}
\mathrm{K}_{1}(F) & \rightarrow \mathrm{K}_{1}(L) & r_{L / F}: \mathrm{K}_{2}(F) & \rightarrow \mathrm{K}_{2}(L) \\
\{a\} & \mapsto\{a\} & \{a, b\} & \mapsto\{a, b\} .
\end{aligned}
$$

Die letztere Abbildung $r_{L / F}$ heißt Restriktionsabbildung oder Restriktion.

Enthält der Körper $F$ eine primitive $n$-te Einheitswurzel $\zeta$, so ergibt sich ein Zusammenhang zwischen der zweiten K-Gruppe und der Brauergruppe des Körpers. Es definiert dann

$$
\begin{aligned}
R_{n, \zeta}: \mathrm{K}_{2}(F) & \longrightarrow \operatorname{Br}_{n}(F) \\
\{a, b\} & \longmapsto[(a, b, \zeta)]
\end{aligned}
$$

einen wohldefinierten Gruppenhomomorphismus, den sogenannten Normresthomomorphismus. In dessen Kern liegt stets die $n$-Torsionsuntergruppe der zweiten K-Gruppe, sodass wir auch einen Morphismus von dem Quotienten $\mathrm{K}_{2}(F) / n \mathrm{~K}_{2}(F)$ in die Brauergruppe erhalten, siehe etwa [Ker90, 19.4 Satz].

Hauptsatz 2.3.15 (Merkurjev-Suslin). Sei $n=p^{m}$ eine Primzahlpotenz und sei $F$ ein Körper, der eine primitive $p^{m}$-te Einheitswurzel enthalte. Dann definiert der Normresthomomorphismus eine Gruppenisomorphie

$$
\mathrm{K}_{2}(F) / p^{m} \mathrm{~K}_{2}(F) \cong \operatorname{Br}_{p^{m}}(F) .
$$

Der Beweis dieser Aussage ist einer der zentralen Punkte bei Kersten [Ker90, 24.2 Theorem] oder auch bei Gille und Szamuely [GS06, Ch. 8].

Für eine Körpererweiterung $L / F$ vertauschen Normresthomomorphismus und Restriktion miteinander:

Lemma 2.3.16. Sei $L / F$ eine Körpererweiterung. Sei $n \in \mathbb{N}$ mit $\operatorname{char}(F) \nmid n$ und es enthalte $F$ eine primitive $n$-te Einheitswurzel $\zeta$. Dann kommutiert das Diagramm

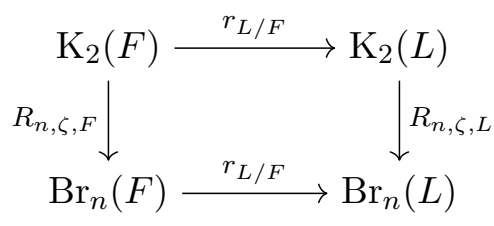


der Normresthomomorphismen $R_{n, \zeta, F}$ und $R_{n, \zeta, L}$ und der beiden Restriktionen $r_{L / F}: \mathrm{K}_{2}(F) \rightarrow \mathrm{K}_{2}(L)$ und $r_{L / F}: \operatorname{Br}(F) \rightarrow \operatorname{Br}(L),[A] \mapsto\left[A \otimes_{F} L\right]$.

Beweis. Siehe etwa [Str02, Lemma 93/5.4].

Wir betrachten die K-Theorie und anschließend auch die Brauergruppe eines diskret bewerteten Körpers:

Definition. Sei $F$ ein diskret bewerteter Körper mit Bewertung $v: F^{\times} \rightarrow \mathbb{Z}$, Restklassenkörper $k$ und einer Uniformisierenden $\pi$. Der Gruppenhomomorphismus

$$
\begin{aligned}
\partial_{v}: \mathrm{K}_{2}(F) & \longrightarrow \mathrm{K}_{1}(k) \\
\{a, b\} & \longmapsto v(a) v(b)\{-1\}+v(b)\left\{\overline{u_{a}}\right\}-v(a)\left\{\overline{u_{b}}\right\}
\end{aligned}
$$

für $a=u_{a} \pi^{v(a)}, b=u_{b} \pi^{v(b)} \in F^{\times}$heißt zahmes Symbol.

Wir werden fortan $\mathrm{K}_{1}(k)$ wieder als multiplikative Einheitengruppe $k^{\times}$schreiben. In dieser Notation lautet das zahme Symbol:

$$
\begin{aligned}
\partial_{v}: \mathrm{K}_{2}(F) & \longrightarrow k^{\times} \\
\{a, b\} & \longmapsto(-1)^{v(a) v(b)} \overline{\left(\frac{a^{v(b)}}{b^{v(a)}}\right)} .
\end{aligned}
$$

Es gilt der folgende Zusammenhang zwischen K-Gruppen und der Brauergruppe:

Satz 2.3.17. Sei $F$ ein diskret bewerteter Körper mit Bewertungsring $R$ und Restklassenkörper $k$, und sei $G=\operatorname{Gal}\left(k_{\mathrm{s}} / k\right)$ dessen absolute Galoisgruppe. Sei $n \in \mathbb{N}$ mit $\operatorname{char}(k) \nmid n$ und es enthalte $F$ eine primitive $n$-te Einheitswurzel $\zeta$. Außerdem sei

$$
t_{\zeta}: \mathrm{H}^{1}\left(G, \frac{1}{n} \mathbb{Z} / \mathbb{Z}\right) \rightarrow \mathrm{H}^{1}\left(G, \mu_{n}\right)
$$

der durch

$$
\frac{1}{n} \mathbb{Z} / \mathbb{Z} \rightarrow \mu_{n}, \quad \frac{i}{n}+\mathbb{Z} \mapsto \zeta^{i}
$$

induzierte Isomorphismus. Dann kommutiert das Diagramm

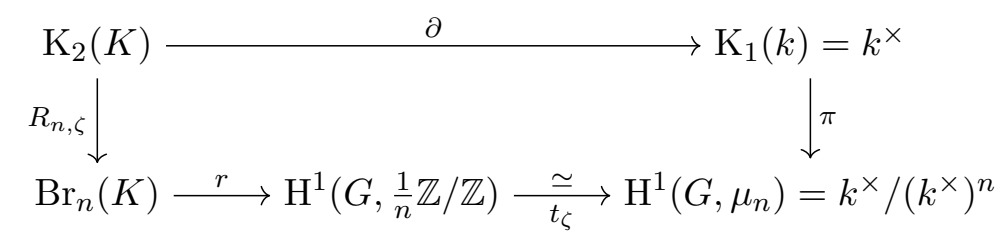

mit der kanonischen Projektion $\pi: k^{\times} \rightarrow k^{\times} /\left(k^{\times}\right)^{n}$ und der Verzweigungsabbildung $r: \operatorname{Br}(K) \rightarrow \mathrm{H}^{1}(G, \mathbb{Q} / \mathbb{Z})$. 
BEweIs. Siehe etwa [Str02, Satz 96/5.7].

In diesem Diagramm sind weder der Normresthomomorphismus $R_{n, \zeta}$ noch der Isomorphismus $t_{\zeta}$ kanonische Abbildungen, beide hängen von der Auswahl der primitiven Einheitswurzel $\zeta$ ab. Wir haben die Abbildung $t_{\zeta}$ gerade so gewählt, dass das obige Diagramm genau kommutiert.

Natürlichere Abbildungen ergeben sich, wenn wir die $n$-Torsion $\operatorname{Br}_{n}(K)=$ $\mathrm{H}^{2}\left(G, \mu_{n}\right)$ durch die Gruppe $\mathrm{H}^{2}\left(G, \mu_{n} \otimes_{\mathbb{Z}} \mu_{n}\right)$ und die Abbildung $R_{n, \zeta}$ durch die vom Cup-Produkt

$$
\begin{aligned}
\cup: \mathrm{H}^{1}\left(G, \mu_{n}\right) \times \mathrm{H}^{1}\left(G, \mu_{n}\right) & \rightarrow \mathrm{H}^{2}\left(G, \mu_{n} \otimes_{\mathbb{Z}} \mu_{n}\right) \\
([f],[h]) & \mapsto[(\sigma, \tau) \mapsto f(\sigma) \otimes \sigma h(\tau)]
\end{aligned}
$$

induzierte Abbildung ersetzen. Man erhält dann das bis auf Vorzeichen kommutierende Diagramm:

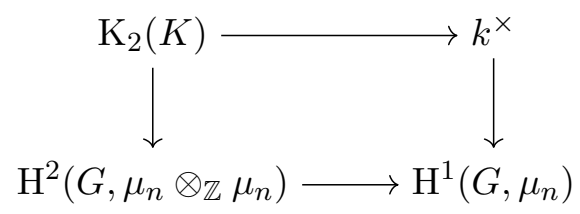

Ein BeweIs findet sich bei Suslin [Sus85, Lemma 18.4].

Für die Frage der Fortsetzbarkeit einer Azumaya-Algebra ergibt sich damit die folgende Aussage:

Satz 2.3.18. Sei $R$ ein diskreter Bewertungsring mit Quotientenkörper $K$ und Restklassenkörper $k$. Es sei $n \in \mathbb{N}$ mit $\operatorname{char}(k) \nmid n$ und es enthalte $K$ eine primitive $n$-te Einheitswurzel $\zeta$. Es seien $a, b$ zwei Einheiten in $K^{\times}$.

Dann ist die Normrestalgebra $(a, b, \zeta)$ genau dann über $R$ fortsetzbar, wenn das zahme Symbol $\partial(\{a, b\})$ eine $n$-te Potenz im Restklassenkörper $k^{\times}$ist.

BeweIs. Sei $A=(a, b, \zeta)_{K}$ eine Normrestalgebra mit $a, b \in K^{\times}$. Da Normresthomomorphismus und Restriktion vertauschen, gilt

$$
\begin{aligned}
\hat{A} & =A \otimes_{K} \hat{K}=(a, b, \zeta)_{K} \otimes_{K} \hat{K}=R_{n, \zeta, K}\left(\{a, b\}_{K}\right) \otimes_{K} \hat{K} \\
& =R_{n, \zeta, \hat{K}}\left(\{a, b\}_{\hat{K}}\right)=(a, b, \zeta)_{\hat{K}} .
\end{aligned}
$$

Da $K$ und seine Vervollständigung denselben Restklassenkörper besitzen, ist das Element, das das zahme Symbol liefert, nicht davon abhängig, ob $\{a, b\}$ als Element von $\mathrm{K}_{2}(K)$ oder als Element von $\mathrm{K}_{2}(\hat{K})$ betrachtet wird. Mit Satz 2.3.17 und Satz 2.3.13 ergibt sich die Behauptung. 


\subsubsection{Ausdehnung als Garbe von Quaternionenalgebren}

Sei $n \in \mathbb{N}$ und sei $F$ ein Körper mit $\operatorname{char}(F) \nmid n$, der eine primitive $n$-te Einheitswurzel $\zeta$ enthalte. Ist $X$ eine reguläre, ganze algebraische Fläche über $F$ und sind $a, b \in F^{\times}$zwei Einheiten, so folgt aus dem letzten Satz und aus Hauptsatz 2.3.14, dass die Normrestalgebra $(a, b, \zeta)$ maximal auf die offene Menge

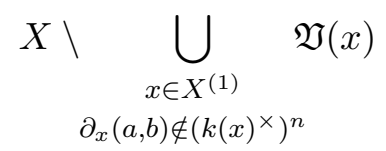

als Garbe von Azumaya-Algebren fortsetzbar ist.

Im Folgenden wollen wir explizit angeben, wie die Konstruktion einer Ausdehnung im Falle einer Quaternionenalgebra aussehen kann. Seien daher von nun an $n=2, F$ ein Körper von Charakteristik $\neq 2, X$ eine reguläre, ganze, projektive Fläche über $F$ mit Funktionenkörper $k(X)$, und sei $A=(a, b)$ die Quaternionenalgebra zu zwei Einheiten $a, b \in k(X)^{\times}$.

Sei $w \in k(X)^{\times}$eine Einheit. Dann heißen

$$
\begin{aligned}
\mathcal{P}(w) & :=\left\{x \in X \mid w \notin \mathcal{O}_{X, x}\right\} \\
\mathcal{N}(w) & :=\left\{x \in X \mid w^{-1} \notin \mathcal{O}_{X, x}\right\}
\end{aligned}
$$

der Polstellendivisor bzw. der Nullstellendivisor von $w$. Dabei werden für die Überprüfung $w, w^{-1} \notin \mathcal{O}_{X, x}$ die lokalen Ringe $\mathcal{O}_{X, x}$ als Teilmenge des Funktionenkörpers $k(X)$ betrachtet.

Für die Punkte $x \in X^{(1)}$ der Kodimension 1 gilt

$$
\begin{aligned}
x \in \mathcal{P}(w) & \Leftrightarrow v_{x}(w)<0, \\
x \in \mathcal{N}(w) & \Leftrightarrow v_{x}(w)>0 \Leftrightarrow w \in \mathfrak{m}_{x}
\end{aligned}
$$

und für die Punkte $x \in X$ beliebiger Kodimension

$$
\begin{aligned}
x \in \mathcal{N}(w) \backslash \mathcal{P}(w) & \Leftrightarrow\left(w \in \mathcal{O}_{X, x} \text { und } w^{-1} \notin \mathcal{O}_{X, x}\right) \Leftrightarrow w \in \mathfrak{m}_{x}, \\
x \notin \mathcal{P}(w) \cup \mathcal{N}(w) & \Leftrightarrow\left(w \in \mathcal{O}_{X, x} \text { und } w^{-1} \in \mathcal{O}_{X, x}\right) \Leftrightarrow w \in \mathcal{O}_{X, x}^{\times} .
\end{aligned}
$$

\section{Ausdehnung auf eine Grundmenge}

Auf der gesamten Fläche $X$ definiert

$$
\mathcal{A}=\mathcal{O}_{X} \oplus \mathcal{O}_{X} i \oplus \mathcal{O}_{X} j \oplus \mathcal{O}_{X} i j
$$

mit den Unbestimmten $i$ und $j$ einen freien, also insbesondere kohärenten $\mathcal{O}_{X}$-Modul der Dimension 4. 
Sei $V=X \backslash(\mathcal{P}(a) \cup \mathcal{P}(b))$. Für jedes $x \in V$ sind dann $a$ und $b$ Elemente in $\mathcal{O}_{X, x}$ und somit auch in $\mathcal{O}_{V}(W)$ für alle offenen Mengen $W \subset V$. Daher definieren die Multiplikationsregeln

$$
i^{2}=a, \quad j^{2}=b, \quad j i=-i j
$$

eine Algebrenstruktur auf der auf $V$ eingeschränkten Garbe $\left.\mathcal{A}\right|_{V}$ auf dem offenen Unterschema $\left(V, \mathcal{O}_{V}\right)$.

Sei

$$
U=X \backslash(\mathcal{P}(a) \cup \mathcal{P}(b) \cup \mathcal{N}(a) \cup \mathcal{N}(b)) .
$$

Für jeden Punkt $x \in U$ sind dann $a$ und $b$ Einheiten in $\mathcal{O}_{X, x}^{\times}$und folglich ist $\left(\mathcal{A}_{V}\right)_{x}=\mathcal{A}_{x}$ eine Azumaya-Algebra über $\mathcal{O}_{X, x}$. Daher definiert $\left.\mathcal{A}\right|_{U}$ eine Garbe von Azumaya-Algebren auf dem offenen Unterschema $\left(U, \mathcal{O}_{U}\right)$, die zudem die Quaternionenalgebra $(a, b)$ fortsetzt.

\section{Ausdehnung auf Teile von Pol- und Nullstellendivisor}

Sei $x \in(\mathcal{P}(a) \cup \mathcal{P}(b) \cup \mathcal{N}(a) \cup \mathcal{N}(b))^{(1)}$ ein Punkt von Kodimension 1, in dem die Quaternionenalgebra $(a, b)$ unverzweigt ist.

Nach Wahl von $x$ aus dem Komplement von $U$ gilt insbesondere

$$
v_{x}(a) \neq 0 \quad \text { oder } \quad v_{x}(b) \neq 0 \text {. }
$$

Da $(a, b)$ in $x$ unverzweigt ist, existiert jedoch eine Ausdehnung von $(a, b)$ als Azumaya-Algebra über $x$, auch wenn dies nicht die bislang konstruierte Garbe $\mathcal{A}$ ist. Um diese zu konstruieren, berechnen wir Elemente $a^{\prime}, b^{\prime} \in k(X)^{\times}$ mit $v_{x}\left(a^{\prime}\right)=v_{x}\left(b^{\prime}\right)=0$ und $(a, b) \cong\left(a^{\prime}, b^{\prime}\right)$.

Für alle $c \in k(X)^{\times}$gilt $\left\{a c^{2}, b\right\}=\{a, b\}+2\{c, b\}$ in der zweiten K-Gruppe. Für die zugehörigen Quaternionenalgebren ergibt sich somit $\left(a c^{2}, b\right) \cong(a, b)$ über $k(X)$ und für das zahme Symbol gilt

$$
\partial_{x}\left(\left\{a c^{2}, b\right\}\right)=\partial_{x}(\{a, b\}) \partial_{x}(\{c, b\})^{2} \equiv \partial_{x}(\{a, b\}) \bmod \left(k(x)^{\times}\right)^{2} .
$$

Nach Multiplikation von $a$ bzw. $b$ mit geraden Potenzen $\pi_{x}^{2 m}$ (mit $m \in \mathbb{Z}$ ) einer Uniformisierenden $\pi_{x} \in \mathcal{O}_{X, x} \subset k(X)$ können wir uns daher ohne Beschränkung der Allgemeinheit auf $v_{x}(a), v_{x}(b) \in\{0,1\}$ einschränken.

Im Fall $v_{x}(a)=v_{x}(b)=1$ ergibt sich zudem $\{a, b\}=\{-b, b\}+\left\{-\frac{a}{b}, b\right\}=$ $\left\{-\frac{a}{b}, b\right\}$ und somit $(a, b) \cong\left(-\frac{a}{b}, b\right)$. Es ist dann $v_{x}\left(-\frac{a}{b}\right)=0$, und daher reicht es insgesamt, den Fall

$$
v_{x}(a)=0 \quad \text { und } \quad v_{x}(b)=1
$$

$\mathrm{zu}$ betrachten. 
Es ist dann $\pi:=b$ eine Uniformisierende für $\mathcal{O}_{X, x}$ und $\partial_{x}(\{a, b\})=\bar{a}$ ist nach Voraussetzung ein Quadrat in $k(x)$. Daher existieren $z, y \in \mathcal{O}_{X, x}$ mit $a=$ $z^{2}-y \pi$. Es ist dann $v_{x}(z)=v_{x}(a)=0$. Da dann auch $a=(z+\pi)^{2}-(2 z+y+\pi) \pi$ und $v_{x}(z+\pi)=v_{x}(z)=0$ gelten und da mindestens eines der beiden Elemente $y$ und $2 z+y+\pi$ Bewertung 0 hat, existieren Elemente $u, w \in \mathcal{O}_{X, x}$ mit

$$
a=u^{2}-w \pi, \quad b=\pi, \quad v_{x}(u)=v_{x}(w)=0 .
$$

Einfache Umformungen ergeben $\{a, b\}-\{a, w\} \in 2 \mathrm{~K}_{2}(K)$. Damit sind die beiden Quaternionenalgebren $(a, b)$ und $(a, w)$ isomorph.

Auf $U_{a, w}=X \backslash(\mathcal{P}(a) \cup \mathcal{P}(w) \cup \mathcal{N}(a) \cup \mathcal{N}(w))$ definiert somit

$$
\mathcal{A}_{a, w}=\mathcal{O}_{U_{a, w}} \oplus \mathcal{O}_{U_{a, w}} i \oplus \mathcal{O}_{U_{a, w}} j \oplus \mathcal{O}_{U_{a, w}} i j
$$

mit den Multiplikationsregeln $i^{2}=a, j^{2}=w, j i=-i j$ eine Garbe von Azumaya-Algebren, die eine Ausdehnung von $A=(a, b) \cong(a, w)$ ist.

\section{Verkleben der beiden Ausdehnungen}

Auf den offenen Mengen $U_{a, b}:=U$ und $U_{a, w}$ definieren $\mathcal{A}_{a, b}:=\left.\mathcal{A}\right|_{U}$ und $\mathcal{A}_{a, w}$ jeweils Ausdehnungen von $A=(a, b) \cong(a, w)$. Wir zeigen noch, dass diese zu einer gemeinsamen Garbe auf $U_{a, b} \cup U_{a, w}$ verkleben.

In der Quaternionenalgebra $(a, b)$ bilden neben $1, i, j, i j$ auch die vier Elemente $1, i, j^{\prime}:=(u-i) b^{-1} j$ und $i j^{\prime}$ eine Basis. Außerdem gilt $\left((u-i) b^{-1} j\right)^{2}=w$. Folglich kann auch $(a, w)$ als Teilmenge von $A$ aufgefasst werden und der Isomorphismus $(a, b) \cong(a, w)$ ist nur ein Basiswechsel mit Matrix

$$
B=\left(\begin{array}{rrrr}
1 & & & \\
& 1 & & \\
& & \frac{u}{\pi} & -\frac{a}{\pi} \\
& & -\frac{1}{\pi} & \frac{u}{\pi}
\end{array}\right)
$$

und Determinante $\operatorname{det}(B)=\frac{w}{\pi}$. Für alle Punkte $z$ im Durchschnitt $U_{a, b} \cap U_{a, w}$ gilt $B \in \mathbb{M}_{4}\left(\mathcal{O}_{X, z}\right)$ und $\operatorname{det}(B) \in \mathcal{O}_{X, z}^{\times}$, also ist $B \in \mathrm{GL}_{4}\left(\mathcal{O}_{X, z}\right)$. Daher gilt

$$
\left.\mathcal{A}_{a, b}\right|_{U_{a, b} \cap U_{a, w}}=\left.\mathcal{A}_{a, w}\right|_{U_{a, b} \cap U_{a, w}}
$$

(quasi als Teilmengen von $A$ ), sodass die beiden Garben $\mathcal{A}_{a, b}$ und $\mathcal{A}_{a, w}$ zu einer gemeinsamen Garbe auf der Vereinigung $U_{a, b} \cup U_{a, w}$ verkleben.

Fahren wir so fort, erhalten wir eine Garbe von Azumaya-Algebren auf einem offenen Unterschema von $X$, das zumindest alle Punkte von Kodimension 1, auf die eine Ausdehnung möglich ist, enthält. Eventuell werden mit dieser Methode jedoch endlich viele abgeschlossene Punkte nicht erreicht, obwohl eine Ausdehnung auf sie existiert. 


\section{Beispiel}

Sei nun speziell $X=\mathbb{P}^{2}$ die projektive Ebene über einem algebraisch abgeschlossenen Körper $F$ von Charakteristik $\neq 2$ mit Funktionenkörper $F(x, y)$.

Wir betrachten zwei Fälle, in denen die Vereinigung

$$
W_{a, b}:=\mathcal{P}(a) \cup \mathcal{P}(b) \cup \mathcal{N}(a) \cup \mathcal{N}(b)
$$

der Pol- und Nullstellendivisoren von $a$ und $b$ aus genau vier Geraden besteht.

Sei zuerst $W_{a, b}$ die Vereinigung von $\mathfrak{V}(x), \mathfrak{V}(y), \mathfrak{V}(x-y)$ und der unendlich fernen Geraden. Die drei affinen Geraden schneiden sich in einem gemeinsamen Punkt und wir können sie zum Beispiel wie folgt auf $a$ und $b$ verteilen:

\begin{tabular}{cccccc}
\hline$a$ & $b$ & $\partial_{(y)}(\{a, b\})$ & $\partial_{(x)}(\{a, b\})$ & $\partial_{(x-y)}(\{a, b\})$ & $\partial_{\infty}(\{a, b\})$ \\
\hline$y$ & $x(x-y)$ & $\left(\frac{1}{x}\right)^{2}$ & $y$ & $y$ & $\frac{x}{y}\left(\frac{x}{y}-1\right)$ \\
1 & $x y(x-y)$ & 1 & 1 & 1 & 1 \\
\hline
\end{tabular}

Im ersten Fall ist die Quaternionenalgebra $(y, x(x-y))$ weder in die beiden Geraden $\mathfrak{V}(x), \mathfrak{V}(x-y)$ noch in die unendlich ferne Gerade fortsetzbar. In die Gerade $\mathfrak{V}(y)$ hingegen ist sie fortsetzbar. Im zweiten Fall ist die Quaternionenalgebra $(1, x y(x-y))$ in alle vier Geraden fortsetzbar, es handelt sich um die zerfallende Algebra.

Sei nun $W_{a, b}$ die Vereinigung der drei parallelen Geraden $\mathfrak{V}(x), \mathfrak{V}(x-1)$, $\mathfrak{V}(x-d)$ und der unendlich fernen Geraden. Diese vier Geraden schneiden sich in einem gemeinsamen Punkt auf der unendlich fernen Geraden.

\begin{tabular}{cccccc}
\hline$a$ & $b$ & $\partial_{(x)}(\{a, b\})$ & $\partial_{(x-1)}(\{a, b\})$ & $\partial_{(x-d)}(\{a, b\})$ & $\partial_{\infty}(\{a, b\})$ \\
\hline$x$ & $(x-1)(x-d)$ & $d^{-1}$ & 1 & $d$ & 1 \\
\hline
\end{tabular}

Die Quaternionenalgebra $(x,(x-1)(x-d))$ ist dann in alle vier Geraden fortsetzbar, es handelt sich auch hier um die zerfallende Algebra.

\subsection{Konstruktion mit dem Cyclic-Covering-Trick}

Chan und Kulkarni stellen in ihren Artikeln [Cha05, CK11] (vor allem im erstgenannten) eine Methode vor, wie man größere Klassen von Maximalordnungen auf algebraischen Flächen explizit und global konstruieren kann. Sie konstruieren diese Maximalordnungen mit Hilfe von nichtkommutativen zyklischen Überlagerungen und betrachten ihre Methode als nichtkommutative Verallgemeinerung eines Tricks mit einer zyklischen Überlagerung (cyclic covering trick im Englischen). Grob zusammengefasst wird auf einer zyklischen Überlagerung $\left(Y, \mathcal{O}_{Y}\right)$ einer algebraischen Fläche eine nichtkommutative Garbe 
mittels eines Bimoduls $\mathcal{L}$ und eines Isomorphismus $\mathcal{L}^{\otimes e} \rightarrow \mathcal{O}_{Y}$ gegeben. Der Bimodul dient dabei als nichtkommutatives Analogon eines Vektorbündels. Letzteres wurde von Artin und Van den Bergh in [AVB90] eingeführt.

Wir wollen für diese globale und daher trotz aller Explizitheit doch recht abstrakte Konstruktion im folgenden Abschnitt das lokale Verhalten genauer untersuchen: Wie sieht die Algebra über dem generischen Punkt aus, wie über den Punkten von Kodimension 1 und wo ist sie Azumaya-Algebra beziehungsweise wo verzweigt sie?

Wir beginnen vorbereitend damit, zyklische Algebren über Körpern ebenfalls mittels eines in der Algebra enthaltenen Bimoduls zu beschreiben und anschließend die entsprechende Konstruktion auf Ringe zu übertragen. Diese nutzen wir dann, um eine Garbe von Maximalordnungen durch Verkleben solcher lokal gegebenen zyklischen Algebren zu konstruieren, und vergleichen diese Methode mit dem Cyclic-Covering-Trick von Chan. Abschließend untersuchen wir noch ein kurz bei Chan angesprochenes Beispiel genauer.

\subsubsection{Zyklische Algebren über Körpern und Bimoduln}

Sei $K^{\prime} / K$ eine zyklische galoissche Körpererweiterung und sei $\sigma$ ein erzeugendes Element ihrer Galoisgruppe $G=\operatorname{Gal}\left(K^{\prime} / K\right) \cong \mathbb{Z} / n \mathbb{Z}$. Zu einer Einheit $a \in K^{\times}$ sei

$$
A=\left(K^{\prime}, \sigma, a\right)
$$

das zyklisch verschränkte Produkt mit Erzeuger $u$ wie in Abschnitt 1.3.1 definiert: Als $K^{\prime}$-Linksmodul ist

$$
A=\bigoplus_{i=0}^{n-1} K^{\prime} u^{i}=K^{\prime} \oplus K^{\prime} u \oplus K^{\prime} u^{2} \oplus \ldots \oplus K^{\prime} u^{n-1},
$$

und die Multiplikation wird durch die beiden Regeln

$$
u^{n}=a, \quad u \lambda=\sigma(\lambda) u
$$

für alle $\lambda \in K^{\prime}$ bestimmt.

Es sei $L=K^{\prime} u$. Dann ist $L$ offensichtlich ein freier $K^{\prime}$-Linksmodul von Rang 1, also ein invertierbarer Modul über $K^{\prime}$. Explizit ist die Skalarmultiplikation von $\lambda \in K^{\prime}$ auf $\ell=\mu u \in L$ (für $\mu \in K^{\prime}$ ) als $\lambda . \ell:=\lambda \ell=(\lambda \mu) u$ gegeben. Durch Einbettung von $L$ in $A$ können wir auch eine $K^{\prime}$-Rechtsmultiplikation auf $L$ definieren, nämlich $\ell \cdot \lambda:=\mu u \cdot \lambda u^{0}=\sigma(\lambda) \mu u=\sigma(\lambda) \ell$, also

$$
\ell \cdot \lambda=\sigma(\lambda) \ell
$$

für alle $\lambda \in K^{\prime}$ und $\ell \in L$. Da Links- und Rechtsmodulstruktur miteinander verträglich sind, d. h. es gilt

$$
\left(\lambda_{1} \cdot \ell\right) \cdot \lambda_{2}=\lambda_{1} \cdot\left(\ell \cdot \lambda_{2}\right)
$$


für alle $\lambda_{1}, \lambda_{2} \in K^{\prime}$ und $\ell \in L$, definieren sie zusammen eine $K^{\prime}$-Bimodulstruktur auf $L$.

Ebenso erhält man auch auf den anderen Summanden $K^{\prime} u^{i}$ eine $K^{\prime}$-Bimodulstruktur: Die Rechtsmultiplikation lautet

$$
\mu u^{i} \cdot \lambda=\sigma^{i}(\lambda) \mu u^{i}
$$

für alle $\mu u^{i} \in K^{\prime} u^{i}$ und $\lambda \in K^{\prime}$.

Für den $K^{\prime}$-Bimodul $L$ lässt sich das wohldefinierte Tensorprodukt $L \otimes_{K^{\prime}} L$, zunächst eine abelsche Gruppe, definieren. Dieses trägt durch Multiplikation des ersten Faktors von links bzw. des zweiten Faktors von rechts mit Elementen aus $K^{\prime}$ ebenfalls eine $K^{\prime}$-Links- und eine $K^{\prime}$-Rechtsmodulstruktur, nämlich

$$
\begin{aligned}
\lambda .\left(\ell_{1} \otimes \ell_{2}\right) & =\left(\lambda \ell_{1}\right) \otimes \ell_{2} \\
\left(\ell_{1} \otimes \ell_{2}\right) . \lambda & =\ell_{1} \otimes\left(\ell_{2} \lambda\right)
\end{aligned}
$$

für alle $\lambda \in K^{\prime}$ und $\ell_{1}, \ell_{2} \in L$. Auch hier sind die beiden Strukturen miteinander verträglich und $L \otimes_{K^{\prime}} L$ kann als $K^{\prime}$-Bimodul betrachtet werden; konkret gilt

$$
\left(\ell_{1} \otimes \ell_{2}\right) \cdot \lambda=\ell_{1} \otimes\left(\sigma(\lambda) \ell_{2}\right)=\left(\ell_{1} \sigma(\lambda)\right) \otimes \ell_{2}=\sigma^{2}(\lambda) \cdot\left(\ell_{1} \otimes \ell_{2}\right)
$$

für alle $\lambda \in K^{\prime}$ und $\ell_{1}, \ell_{2} \in L$.

Die Abbildung $L \times L \rightarrow A,\left(\ell_{1}, \ell_{2}\right) \mapsto \ell_{1} \ell_{2}$ ist bilinear über dem Grundkörper $K$. Da die Multiplikation in $A$ assoziativ ist, folgt $\left(\ell_{1} \lambda\right) \ell_{2}=\ell_{1}\left(\lambda \ell_{2}\right)$ für alle $\ell_{1}, \ell_{2} \in L$ und $\lambda \in K^{\prime}$, und man kann die kanonische bilineare Paarung des Tensorproduktes

$$
\begin{aligned}
\varphi: L \otimes_{K^{\prime}} L & \rightarrow A \\
\ell_{1} \otimes \ell_{2} & \mapsto \ell_{1} \ell_{2}
\end{aligned}
$$

betrachten. Offensichtlich gilt für das $\operatorname{Bild} \operatorname{im}(\varphi)=K^{\prime} u^{2} \subset A$; und als nichttriviale, surjektive Abbildung zwischen den freien $K^{\prime}$-Linksmoduln $L \otimes_{K^{\prime}} L$ und $K^{\prime} u^{2}$ von gleichem Rang 1 ist $\varphi$ auch injektiv. Insgesamt erhalten wir daher eine Isomorphie

$$
L \otimes_{K^{\prime}} L \cong K^{\prime} u^{2}
$$

von $K^{\prime}$-Linksmoduln. Da $\varphi$ mit der $K^{\prime}$-Rechtsmultiplikation auf $L \otimes_{K^{\prime}} L$ bzw. auf $K^{\prime} u^{2}$ verträglich ist, ist obiges auch eine Isomorphie von $K^{\prime}$-Bimoduln.

Allgemeiner können wir für $i \leq n-1$ auch das $i$-fache Tensorprodukt

$$
L^{\otimes i}=L \otimes_{K^{\prime}} L \otimes_{K^{\prime}} \ldots \otimes_{K^{\prime}} L \quad(i \text { Faktoren } L)
$$

betrachten. Es gilt dann analog

$$
\left(\ell_{1} \otimes \ldots \otimes \ell_{i}\right) \cdot \lambda=\sigma^{i}(\lambda) \cdot\left(\ell_{1} \otimes \ldots \otimes \ell_{i}\right)
$$


für alle $\ell_{j} \in L$ und $\lambda \in K^{\prime}$, und wir erhalten eine Isomorphie

$$
L^{\otimes i} \cong K^{\prime} u^{i} \subset A
$$

(mit $\left.\ell_{1} \otimes \ell_{2} \otimes \ldots \otimes \ell_{i} \mapsto \ell_{1} \ell_{2} \cdots \ell_{i}\right)$ von $K^{\prime}$-Bimoduln. Die zyklische AzumayaAlgebra $A$ lässt sich damit als

$$
A=K^{\prime} \oplus L \oplus L^{\otimes 2} \oplus \ldots \oplus L^{\otimes n-1}
$$

darstellen.

Im vorliegenden Fall geht die Rechtsmultiplikation auf $L$ mit Hilfe des Automorphismus $\sigma$ aus der Linksmultiplikation hervor. Dies können wir allgemein betrachten:

Definition. Seien $M$ ein $R$-Linksmodul und $\rho$ ein Automorphismus von $R$. Dann sei $M_{\rho}$ derjenige $R$-Bimodul, der aus dem $R$-Linksmodul $M$ mit der zusätzlichen Rechtsmultiplikation $m . \lambda=\rho(\lambda) m$ für alle $\lambda \in R$ und $m \in M$ entsteht.

Es ist $M_{\rho}$ in der Tat ein $R$-Bimodul, denn es gilt $\left(\lambda_{1} m\right) \cdot \lambda_{2}=\lambda_{1}\left(m \cdot \lambda_{2}\right)$ für alle $\lambda_{1}, \lambda_{2} \in R$ und $m \in M$.

Es ist auch möglich, mittels eines Automorphimus eine neue (getwistete) Linksmultiplikation zu definieren:

Definition. Seien $M$ ein $R$-Linksmodul und $\rho$ ein Automorphismus von $R$. Es sei $\rho^{*} M$ derjenige $R$-Linksmodul, der aus der abelschen Gruppe $M$ und der mittels $\rho$ getwisteten Linksmultiplikation $\lambda . m=\rho^{-1}(\lambda) m$ für $\lambda \in R, m \in M$ entsteht.

Seien nun $M_{\rho}$ und $N_{\tau}$ die $R$-Bimoduln zu den $R$-Linksmoduln $M$ und $N$ und den Automorphismen $\rho$ und $\tau$ von $R$.

Da dann $M_{\rho}$ ein $R$-Rechtsmodul und $N_{\tau}$ ein $R$-Linksmodul ist, lässt sich das Tensorprodukt $M_{\rho} \otimes_{R} N_{\tau}$ als abelsche Gruppe definieren. Eine $R$-Linksmultiplikation auf dem Tensorprodukt ergibt sich durch Linksmultiplikation des ersten Faktors. Es gilt dann

$$
\lambda .(m \otimes n)=(\lambda m) \otimes n=\left(m \rho^{-1}(\lambda)\right) \otimes n=m \otimes\left(\rho^{-1}(\lambda) n\right)
$$

für alle $\lambda \in R, m \in M, n \in N$. Wir erhalten somit eine Isomorphie

$$
M_{\rho} \otimes_{R} N_{\tau} \cong M \otimes_{R} \rho^{*} N
$$

von $R$-Linksmoduln. Außerdem lässt sich auf dem Tensorprodukt $M_{\rho} \otimes_{R} N_{\tau}$ wiederum eine $R$-Rechtsmultiplikation durch Multiplikation des rechten Faktors von rechts mit $\lambda \in R$ definieren. Es gilt dann

$$
\begin{aligned}
(m \otimes n) . \lambda & =m \otimes(n \lambda)=m \otimes(\tau(\lambda) n) \\
& =(m \tau(\lambda) \otimes n)=(\rho \tau(\lambda) m) \otimes n \\
& =\rho \tau(\lambda) \cdot(m \otimes n)
\end{aligned}
$$


für alle $\lambda \in R, m \in M, n \in N$. Damit gilt für das Tensorprodukt der beiden Bimoduln die folgende Aussage:

Lemma 2.4.1. Das Tensorprodukt zweier R-Bimoduln $M_{\rho}$ und $N_{\tau}$ ist ebenfalls ein R-Bimodul. Es gilt

$$
M_{\rho} \otimes_{R} N_{\tau} \cong\left(M \otimes_{R} \rho^{*} N\right)_{\rho \tau} .
$$

Wenden wir diese Erkenntnisse und Schreibweisen auf unsere zyklische Algebra $A=\left(K^{\prime}, \sigma, a\right)=K^{\prime} \oplus L \oplus L^{\otimes 2} \oplus \ldots \oplus L^{\otimes n-1}$ an, so müssen wir in der Notation den $K^{\prime}$-Linksmodul $L$ durch den $K^{\prime}$-Bimodul $L_{\sigma}$ ersetzen und erhalten

$$
A=K^{\prime} \oplus L_{\sigma} \oplus L_{\sigma}^{\otimes 2} \oplus \ldots \oplus L_{\sigma}^{\otimes n-1} .
$$

Für die Tensorprodukte gilt dann außerdem

$$
L_{\sigma}^{\otimes i}=\left(L \otimes_{K^{\prime}} \sigma^{*} L \otimes_{K^{\prime}} \ldots \otimes_{K^{\prime}} \sigma^{(i-1) *} L\right)_{\sigma^{i}} .
$$

\subsubsection{Zyklische Algebren über Ringen}

Sei $R$ ein kommutativer Ring mit Eins.

Man kann versuchen, die obige Konstruktion von zyklischen Algebren auf gewisse Ringerweiterungen zu übertragen. In der folgenden Situation ist das leicht möglich.

Sei $R^{\prime}$ eine endliche Ringerweiterung von $R$ und sei $\sigma: R^{\prime} \rightarrow R^{\prime}$ ein $R$-Algebrenisomorphismus von Ordnung $n$. Für die von $\sigma$ erzeugte Untergruppe der $R$-Algebrenisomorphismen von $R^{\prime}$ gilt dann $\operatorname{Aut}\left(R^{\prime} / R\right) \supseteq\langle\sigma\rangle \cong \mathbb{Z} / n \mathbb{Z}$.

Mit Hilfe von $\sigma$ definieren wir über $R^{\prime}$ den Schiefpolynomring

$$
A^{\prime}=\left(R^{\prime} / R\right)[u, \sigma]=\bigoplus_{i=0}^{\infty} R^{\prime} u^{i}
$$

als freien $R^{\prime}$-Linksmodul über der Unbestimmten $u$ mit der Multiplikation, die durch

$$
u x=\sigma(x) u
$$

für alle $x \in R^{\prime}$ und durch $u^{i} u^{j}=u^{i+j}$ für alle $i, j \in \mathbb{N}_{0}$ gegeben ist.

Bemerkung 2.4.2. Für jedes Element $\ell \in R^{\prime} u \subset A^{\prime}$ gilt ebenfalls $\ell x=\sigma(x) \ell$ für alle $x \in R^{\prime}$ und die $n$-te Potenz $\ell^{n}$ liegt im Zentrum $Z\left(A^{\prime}\right)$ von $A^{\prime}$.

Beweis. Sei $\ell=y u \in R^{\prime} u$. Für jedes $x \in R^{\prime}$ gilt $\ell x=y u x=\sigma(x) y u=\sigma(x) \ell$. Zum einen ist dann $\ell^{n} x=x \ell^{n}$, da $\sigma$ Ordnung $n$ hat, und zum anderen gilt

$$
\begin{aligned}
u \ell^{n} & =u(y u)^{n}=u\left(y \sigma(y) \cdots \sigma^{n-1}(y)\right) u^{n} \\
& =\left(\sigma(y) \sigma^{2}(y) \cdots \sigma^{n}(y)\right) u u^{n}=\left(y \sigma(y) \cdots \sigma^{n-1}(y)\right) u^{n} u \\
& =\ell^{n} u .
\end{aligned}
$$

Insgesamt vertauscht $\ell^{n}$ damit mit allen Elementen aus $A^{\prime}$. 
Es sei zusätzlich ein Element $a \in R$ gegeben. Wir betrachten dann die Quotientenalgebra von $A^{\prime}$ modulo $u^{n}-a$ : Es sei

$$
A=\left(R^{\prime} / R, \sigma, a\right)=A^{\prime} /\left(u^{n}-a\right) .
$$

Da die Potenzen $u^{i}$ für $i=0,1, \ldots, n-1$ weiterhin linear unabhängig sind, ist $A$ als $R^{\prime}$-Linksmodul frei von konstantem Rang $n$ und wir können die Algebra auch folgendermaßen charakterisieren: Es sei

$$
A=\left(R^{\prime} / R, \sigma, a\right)=\bigoplus_{i=0}^{n-1} R^{\prime} u^{i}
$$

als freier $R^{\prime}$-Linksmodul von Rang $n$ über der Unbestimmten $u$. Die Multiplikation auf $A$ sei durch die Regeln

$$
u x=\sigma(x) u \quad \text { und } \quad u^{n}=a
$$

für alle $x \in R^{\prime}$ und durch $u^{i} u^{j}=u^{i+j}$ gegeben.

\section{Charakterisierung der Summanden}

Die direkten Summanden $R^{\prime} u^{i}$ von $A$ lassen sich gewissermaßen durch die Wirkung von $\sigma$ beschreiben:

Lemma 2.4.3. Sei $R^{\prime}$ nullteilerfrei. Dann gilt

$$
R^{\prime} u^{i}=\left\{z \in A \mid z x=\sigma^{i}(x) z \forall x \in R^{\prime}\right\}
$$

für $i=0,1, \ldots, n-1$. Insbesondere gilt also, dass $R^{\prime}$ sein eigener Zentralisator in $A$ ist und dass

$$
R^{\prime} u=\left\{z \in A \mid z x=\sigma(x) z \forall x \in R^{\prime}\right\}
$$

ist.

BeweIs. Es sei $i \in\{0,1, \ldots, n-1\}$ und $z=\sum_{j=0}^{n-1} y_{j} u^{j}$ so, dass $z x=\sigma^{i}(x) z$ für alle $x \in R^{\prime}$ gilt. Dann ist

$$
\sum_{j} y_{j} \sigma^{j}(x) u^{j}=\sum_{j} y_{j} u^{j} x=z x=\sigma^{i}(x) z=\sum_{j} y_{j} \sigma^{i}(x) u^{j},
$$

und da die $u^{j}$ linear unabhängig sind, gilt somit $y_{j}\left(\sigma^{j}(x)-\sigma^{i}(x)\right)=0$ für alle $j=0, \ldots, n-1$ und alle $x \in R^{\prime}$. Da $\sigma$ Ordnung $n$ hat und da $R^{\prime}$ nullteilerfrei ist, folgt $y_{j}=0$ für $j \neq i$ und somit $z \in R^{\prime} u^{i}$. Die umgekehrte Inklusion ergibt sich unmittelbar aus der Definition der Multiplikation. 


\section{Das Zentrum der Algebra}

Korollar. Ist die Ringerweiterung $R^{\prime}$ nullteilerfrei, so ist das Zentrum von $A$ genau der Fixring $\left(R^{\prime}\right)^{\sigma}$ unter der Operation von $\sigma$ auf $R^{\prime}$.

Ist $R^{\prime}$ nullteilerfrei und ist $R$ der Fixring unter der Operation von $\sigma$, gilt also $\left(R^{\prime}\right)^{\sigma}=R$, so ist die Algebra A zentral über $R$.

Beweis. Sei $z$ ein Element des Zentrums von $A$. Dann liegt $z$ insbesondere im Zentralisator von $R^{\prime}$ und stammt nach dem vorhergehenden Lemma somit selbst aus $R^{\prime}$. Außerdem vertauscht $z$ mit der Unbestimmten $u$, also ist $z u=$ $u z=\sigma(z) u$ und somit gilt $z=\sigma(z)$ wie behauptet.

Recht analog folgt, dass das Zentrum von $A^{\prime}$ gerade $Z\left(A^{\prime}\right)=\bigoplus_{i=0}^{\infty} R\left(u^{n}\right)^{i}$ ist, wenn wiederum $R^{\prime}$ nullteilerfrei und $\left(R^{\prime}\right)^{\sigma}=R$ ist.

\section{Darstellung mittels Bimoduln}

Wie über Körpern können wir auch über Ringen der Sichtweise Chans und Kulkarnis ([Cha05, CK11, jeweils Sec. 2]) folgen und den $R^{\prime}$-Linksmodul $R^{\prime} u$, den wir durch willkürliche Wahl einer Unbestimmten $u$ gegeben haben, durch einen beliebigen $R^{\prime}$-Bimodul $L$, der als $R^{\prime}$-Linksmodul frei und von Rang 1 ist und dessen Links- und Rechtsstruktur wie zuvor durch die Anwendung von $\sigma$ auseinander hervorgehen, austauschen.

Die erste Algebra lässt sich dann als $R^{\prime}$-Linksmodul

$$
A^{\prime}=R^{\prime} \oplus L \oplus L^{\otimes 2} \oplus L^{\otimes 3} \oplus \ldots
$$

schreiben. Die Multiplikation ergibt sich durch das Tensorprodukt von Bimoduln und die Vertauschungsbedingung lautet dann

$$
\ell x=\sigma(x) \ell
$$

für alle $x \in R^{\prime}$ und $\ell \in L$.

Haben wir die Beschreibung mit einer Unbestimmten $u$ gegeben, definieren wir $L=R^{\prime} u$ als $R^{\prime}$-Linksmodul und erhalten die Rechtsmultiplikation und damit die Bimodulstruktur wie zuvor aus der angegebenen Multiplikation, also $\ell . x=\sigma(x) \ell$ für $x \in R^{\prime}$ und $\ell \in L$. Haben wir hingegen einen Bimodul $L$ (bzw. in der genauen Notation eigentlich $L_{\sigma}$ ) gegeben, so können wir $u$ als einen beliebigen Erzeuger im Linksmodul $L$ wählen.

Um die eigentlich gewünschte Algebra $A$ zu definieren, muss die Bedingung $u^{n}=a \in R$ durch einen geeigneten Homomorphismus

$$
\varphi: L^{\otimes n} \rightarrow R^{\prime}
$$


ersetzt werden. Dieser Homomorphismus muss eine gewisse zusätzliche Bedingung erfüllen, die bei Chan und Kulkarni ([Cha05, Sec. 3] und [CK11, Sec. 2]) sogenannte Überschneidungsbedingung (overlap condition), nämlich:

$$
1 \otimes \varphi=\varphi \otimes 1
$$

für $1 \otimes \varphi: L^{\otimes n+1}=L \otimes L^{\otimes n} \rightarrow L \otimes R=L$ und $\varphi \otimes 1: L^{\otimes n} \otimes L \rightarrow R \otimes L=L$. Diese sorgt letztlich dafür, dass die Multiplikation wohldefiniert und assoziativ ist.

Die Algebra $A$ ist dann der $R^{\prime}$-Linksmodul

$$
A=\left(R^{\prime} / R, \sigma, a\right)=R^{\prime} \oplus L \oplus L^{\otimes 2} \oplus \ldots \oplus L^{\otimes n-1}
$$

zusammen mit der Multiplikation, die sich aus dem Tensorprodukt, der Vertauschungsregel

$$
\ell x=\sigma(x) \ell
$$

für alle $x \in R^{\prime}, \ell \in L$ und der Anwendung des Homomorphismus $\varphi: L^{\otimes n} \rightarrow R^{\prime}$ ergibt. Letzteres bedeutet insbesondere $\ell^{n}=\varphi(\ell)$ für alle $\ell \in L$.

\section{Zyklizitätsbedingung}

Wir wollen fortan nur Ringerweiterungen $R^{\prime} / R$ betrachten, die in gewisser Weise zyklisch sind.

Es sei daher von nun an zusätzlich Folgendes erfüllt: Es sei $R$ ein Integritätsbereich und es sei $R^{\prime}$ als $R$-Modul projektiv und von konstantem Rang $n$, der Fixring von $R^{\prime}$ unter der Operation von $\sigma$ sei $R$ und es erzeuge $\sigma$ die gesamte Automorphismengruppe $\operatorname{Aut}\left(R^{\prime} / R\right)$. Es gelte also $\left(R^{\prime}\right)^{\sigma}=R$ und $\operatorname{Aut}\left(R^{\prime} / R\right)=\langle\sigma\rangle \cong \mathbb{Z} / n \mathbb{Z}$.

Bemerkung 2.4.4 (Eindeutigkeit der $n$-ten Potenz). Ist $x \in R^{\prime}$ und $\ell \in L$, so gilt

$$
(x \ell)^{n}=x \sigma(x) \sigma^{2}(x) \cdots \sigma^{n-1}(x) \cdot \ell^{n}=\mathrm{N}_{R^{\prime} / R}(x) \ell^{n} .
$$

Zwei Erzeuger $u_{1}$ und $u_{2}$ von $L$ als $R^{\prime}$-Linksmodul unterscheiden sich genau um eine Einheit $\lambda \in R^{\prime \times}$, etwa $u_{1}=\lambda u_{2}$. Mithin ist bei gegebenem $L$ unabhängig von der getroffenen Wahl des Erzeugers $u$ die $n$-te Potenz $a=u^{n}$ eindeutig bestimmt modulo $\mathrm{N}_{R^{\prime} / R}\left(R^{\prime \times}\right)$. Wir betrachten $R^{\prime}$ als multiplikativen Monoid, und es sei

$$
\alpha:=u^{n} \cdot \mathrm{N}_{R^{\prime} / R}\left(R^{\prime \times}\right)
$$

die Restklasse von $a=u^{n}$ in $R / \mathrm{N}_{R^{\prime} / R}\left(R^{\prime \times}\right)$, dem Monoiden der Normenreste.

Die Algebra $A$ lässt sich in diesem Fall bis auf Isomorphie durch die Daten

$$
A=\left(R^{\prime} / R, \operatorname{Aut}\left(R^{\prime} / R\right)=\langle\sigma\rangle, \alpha\right)
$$

vollständig beschreiben. 


\subsubsection{Garben zyklischer Algebren}

Im nächsten Schritt soll die obige Konstruktion garbifiziert werden. Wir betrachten eine endliche Überlagerung mit zyklischer Automorphismengruppe oder allgemeiner mit spezieller zyklischer Untergruppe.

Es sei $k$ ein algebraisch abgeschlossener Körper von Charakteristik 0. Es seien $\left(X, \mathcal{O}_{X}\right)$ und $\left(Y, \mathcal{O}_{Y}\right)$ glatte, ganze Schemata von endlichem Typ über $k$ und es sei

$$
p: Y \rightarrow X
$$

ein endlicher Morphismus von Rang $n$. Es ist dann $\mathcal{O}_{Y}$ als kohärente $\mathcal{O}_{X}$-Modulgarbe lokalfrei von Rang $n$.

Außerdem sei $\sigma \in \operatorname{Aut}(Y / X)$ ein solcher Automorphismus von $Y$ von Ordnung $n$, dass für die Strukturgarben

$$
\left(\mathcal{O}_{Y}\right)^{\sigma}=\mathcal{O}_{X}
$$

oder genau genommen $\left(\mathcal{O}_{Y}\right)^{\sigma}=p^{*}\left(\mathcal{O}_{X}\right)$, gilt. Für die von $\sigma$ erzeugte Untergruppe gilt dann $\operatorname{Aut}(Y / X) \supset\langle\sigma\rangle \cong \mathbb{Z} / n \mathbb{Z}$.

\section{Lokale Konstruktion durch Verklebung}

Sei $\left\{U_{i}=\operatorname{Spec} R_{i} \subset X \mid i \in I\right\}$ eine offene affine Überdeckung von $X$. Die Urbilder der $U_{i}$ bilden dann eine offene Überdeckung von $Y$. Da $p$ ein endlicher Morphismus ist, sind diese Urbilder $p^{-1}\left(U_{i}\right)=\operatorname{Spec} R_{i}^{\prime}$ affine Mengen in $Y$ und jeder Ring $R_{i}^{\prime}$ ist jeweils ein endlich erzeugter projektiver $R_{i}$-Modul von konstantem Rang $n$. Dabei gilt $R_{i}^{\prime}=\Gamma\left(p^{-1} U_{i}, \mathcal{O}_{Y}\right)$. Durch die Wahl genügend kleiner offener affiner Mengen können wir davon ausgehen, dass auch alle paarweisen Durchschnitte affin sind, etwa $U_{i} \cap U_{j}=$ Spec $R_{i j}$ und $p^{-1} U_{i} \cap p^{-1} U_{j}=\operatorname{Spec} R_{i j}^{\prime}$ für alle $i \neq j$, und dass jeweils $R_{i}^{\prime}$ als $R_{i}$-Modul bzw. $R_{i j}^{\prime}$ als $R_{i j}$-Modul nicht nur projektiv, sondern frei von Rang $n$ ist.

Wir erhalten dann die folgenden kommutierenden Diagramme

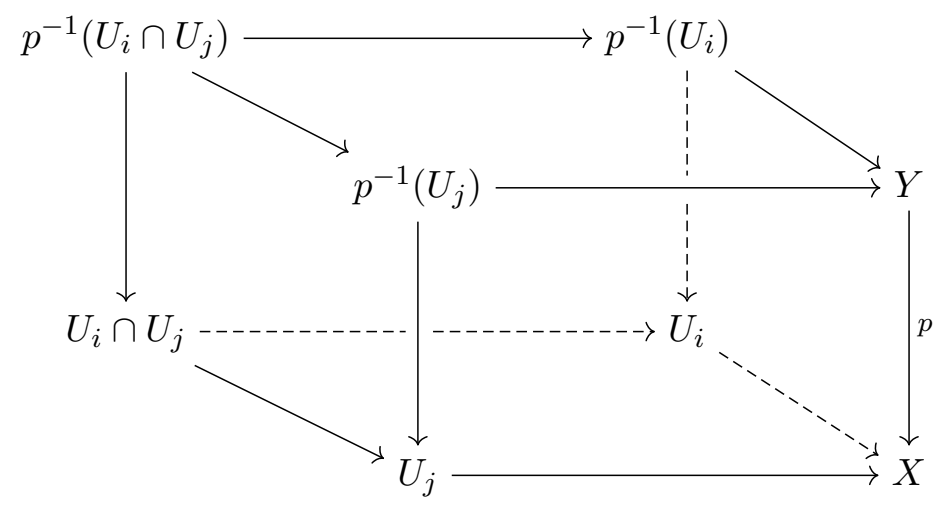


der offenen Mengen - oder offenen Unterschemata - in $X$ und in $Y$ und ihrer zugehörigen Ringe

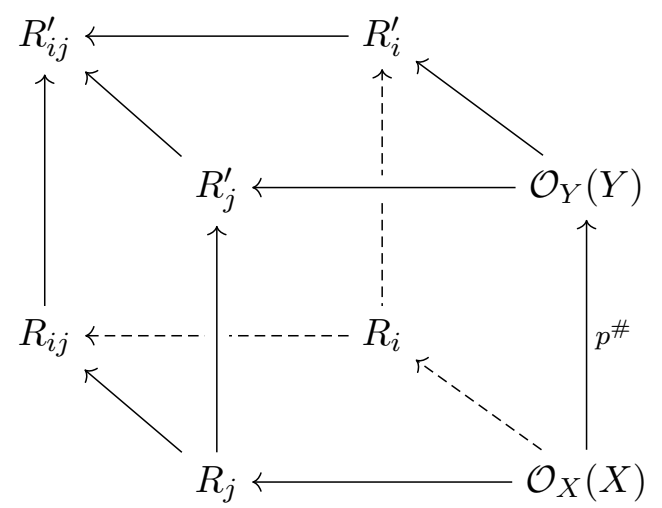

für alle $i \neq j$ aus $I$.

Wir können alle Ringe $R_{i}, R_{i j}, R_{i}^{\prime}$ und $R_{i j}^{\prime}$ als Unterringe des Funktionenkörpers $k(Y)$ auffassen und es gilt

$$
\begin{aligned}
\operatorname{Aut}\left(R_{i}^{\prime} / R_{i}\right) & \supset\langle\sigma\rangle \cong(\mathbb{Z} / n \mathbb{Z}) \quad \text { und } \\
\operatorname{Aut}\left(R_{i j}^{\prime} / R_{i j}\right) & \supset\langle\sigma\rangle \cong(\mathbb{Z} / n \mathbb{Z}) .
\end{aligned}
$$

Die Notation vereinfachend bezeichnen wir dabei auch die Einschränkungen von $\sigma$ auf die offenen Mengen $U \subset X$ und $p^{-1}(U) \subset Y$ mit $\sigma$. Für $U \subset X$ ist der Morphismus trivial, $\left.\sigma\right|_{U}=\operatorname{id}_{U}$. Für die Urbilder $p^{-1}(U)$ in $Y$ gilt $\sigma\left(p^{-1}(U)\right)=p^{-1}(U)$. Letzteres ist für beliebige offene Mengen $V \subset Y$ im Allgemeinen nicht der Fall.

Seien außerdem Elemente $a_{i} \in R_{i} \subset k(X)$ und $\beta_{j i} \in\left(R_{i j}^{\prime}\right)^{\times}$so gegeben, dass

$$
a_{i}=\mathrm{N}_{R_{i j}^{\prime} / R_{i j}}\left(\beta_{i j}\right) a_{j}
$$

in $R_{i j}^{\prime} \subset k(X)$ und $\beta_{i h}=\beta_{i j} \beta_{j h}$ für alle $i, j, h \in I$ gilt. Es gilt dann automatisch $\beta_{i i}=1$ und $\beta_{j i}=\beta_{i j}^{-1}$ für alle $i, j$. Außerdem folgt aus der ersten Bedingung, dass alle Elemente $a_{i}$ dieselbe Restklasse $\alpha$ in $k(X) / N_{k(Y) / k(X)}\left(k(Y)^{\times}\right)$haben. Auf jeder der offenen affinen Mengen $U_{i}^{\prime}=p^{-1}\left(U_{i}\right)=\operatorname{Spec} R_{i}^{\prime}$ definiert die zyklische Algebra

$$
A_{i}=\left(R_{i}^{\prime} / R_{i}, \sigma, a_{i}\right)=\bigoplus_{t=0}^{n-1} R_{i}^{\prime} u_{i}^{t}
$$

mit $u_{i} x=\sigma(x) u_{i}$ für $x \in R_{i}^{\prime}$ und $u_{i}^{n}=a_{i}$ eine $\mathcal{O}_{U_{i}}$-Algebrengarbe $\mathcal{A}_{i}$.

Das kommutierende Diagramm der Ringe $R_{i}, R_{i j}, R_{i}^{\prime}$ und $R_{i j}^{\prime}$ ergibt eine natürliche Abbildung vom Tensorprodukt $R_{i j} \otimes_{R_{i}} R_{i}^{\prime}$ in den Ring $R_{i j}^{\prime}$, nämlich $x \otimes y \mapsto x y$. Dadurch erhalten wir Abbildungen

$$
\psi_{i j}: A_{i}=\left(R_{i}^{\prime} / R_{i}, \sigma, a_{i}\right) \rightarrow R_{i j} \otimes_{R_{i}} A_{i} \rightarrow A_{i j}:=\left(R_{i j}^{\prime} / R_{i j}, \sigma, a_{i}\right)
$$


für alle $i \neq j$. Für diese gilt $\psi_{i j}\left(u_{i}\right)^{m}=\psi_{i j}\left(u_{i}^{m}\right)$ und $\psi_{i j}\left(u_{i} x\right)=\psi_{i j}\left(u_{i}\right) \psi_{i j}(x)$ für alle $x \in R_{i}^{\prime}$ und $m \in \mathbb{N}$. Die $\psi_{i j}$ sind also wohldefiniert und multiplikativ.

Die $R_{i j}^{\prime}$-Linksmodulhomomorphismen $\varphi_{i j}: A_{i j} \rightarrow A_{j i}, \psi_{i j}\left(u_{i}\right) \mapsto \beta_{i j} \psi_{j i}\left(u_{j}\right)$ beziehungsweise ihre Einschränkungen

$$
\begin{aligned}
\varphi_{i j}: R_{i j} \otimes_{R_{i}} A_{i} & \rightarrow R_{i j} \otimes_{R_{j}} A_{j} \\
u_{i} & \mapsto \beta_{i j} u_{j}
\end{aligned}
$$

erfüllen die beiden Bedingungen

$$
\begin{aligned}
\varphi_{i j}\left(u_{i} x\right) & =\varphi_{i j}\left(\sigma(x) u_{i}\right)=\sigma(x) \beta_{i j} u_{j}=\beta_{i j} u_{j} x=\varphi_{i j}\left(u_{i}\right) \varphi_{i j}(x), \\
\varphi_{i j}\left(u_{i}\right)^{n} & =\left(\beta_{i j} u_{j}\right)^{n}=\mathrm{N}_{R_{i j}^{\prime} / R_{i j}}\left(\beta_{i j}\right) u_{j}^{n}=\mathrm{N}\left(\beta_{i j}\right) a_{j}=a_{i} \\
& =\varphi_{i j}\left(a_{i}\right)=\varphi_{i j}\left(u_{i}^{n}\right)
\end{aligned}
$$

für alle $x \in R_{i j}^{\prime}$. Außerdem gilt offensichtlich $\varphi_{i j} \circ \varphi_{j i}=\mathrm{id}$ und $\varphi_{i i}=\mathrm{id}$ für alle $i \neq j$. Folglich ist $\varphi_{i j}$ ein $R_{i j}$-Algebrenisomorphismus und zugleich ein $R_{i j}^{\prime}$-Bimodulisomorphismus von $A_{i j}$ zu $A_{j i}$ bzw. von $R_{i j}^{\prime} \otimes_{R_{i}^{\prime}} A_{i}$ zu $R_{i j}^{\prime} \otimes_{R_{j}^{\prime}} A_{j}$.

All diese Algebrenmorphismen induzieren zusammen Garbenisomorphismen

$$
\varphi_{i j}:\left.\left.\mathcal{A}_{i}\right|_{U_{i}^{\prime} \cap U_{j}^{\prime}} \rightarrow \mathcal{A}_{j}\right|_{U_{i}^{\prime} \cap U_{j}^{\prime}}
$$

auf $U_{i}^{\prime} \cap U_{j}^{\prime}$, für die ebenfalls $\varphi_{i j} \circ \varphi_{j i}=\mathrm{id}$ und $\varphi_{i i}=\mathrm{id}$ sowie

$$
\varphi_{j h} \varphi_{i j}\left(u_{i}\right)=\varphi_{j h}\left(\beta_{i j} u_{j}\right)=\beta_{i j} \beta_{j h} u_{h}=\beta_{i h} u_{h}=\varphi_{i h}\left(u_{i}\right)
$$

und somit $\varphi_{i h}=\varphi_{j h} \circ \varphi_{i j}$ auf $U_{i}^{\prime} \cap U_{j}^{\prime} \cap U_{h}^{\prime}$ gilt.

Folglich verkleben die Garben $\mathcal{A}_{i}$ auf der Überdeckung $\left(U_{i}^{\prime}\right)_{i \in I}$ von $Y$ zu einer $\mathcal{O}_{Y^{-}}$Bimodulgarbe $\mathcal{A}$ auf $Y$ und weiter über Push-forward zu einer $\mathcal{O}_{X^{-}} \mathrm{Al}$ gebrengarbe auf $X$.

Insgesamt ist unsere Garbe $\mathcal{A}$ also durch lokale Daten

$$
\left.\mathcal{A}\right|_{U_{i}}=\left(R_{i} \rightarrow R_{i}^{\prime} \cong R_{i}^{n} ;\langle\sigma\rangle \subset \operatorname{Aut}\left(R_{i}^{\prime} / R_{i}\right) ; \alpha_{i} \in R_{i} / \mathrm{N}_{R_{i}^{\prime} / R_{i}}\left(R_{i}^{\prime \times}\right)\right)
$$

und durch die Verklebebedingungen $a_{i}=\mathrm{N}_{R_{i j}^{\prime} / R_{i j}}\left(\beta_{i j}\right) a_{j}$ mit $\beta_{i h}=\beta_{i j} \beta_{j h}$ für alle $i, j, h \in I$ gegeben, dabei sei $\alpha_{i}$ die Restklasse von $a_{i}$ modulo $\mathrm{N}_{R_{i}^{\prime} / R_{i}}\left(R_{i}^{\prime \times}\right)$. Betrachten wir jeweils die $R_{i}^{\prime}$-Bimoduln $L_{i}=R_{i}^{\prime} u_{i} \subset A_{i}$, so definieren diese $\mathcal{O}_{U_{i}^{\prime}}$-Bimoduln $\mathcal{L}_{i}$ von Rang 1 auf den offenen affinen Mengen $U_{i}^{\prime}$. Über die Einschränkungen der obigen Morphismen $\varphi_{i j}$ verkleben diese zu einem $\mathcal{O}_{Y^{-}}$ Bimodul $\mathcal{L}$ von Rang 1.

Wie zuvor folgt

$$
\mathcal{A}=\mathcal{O}_{Y} \oplus \mathcal{L} \oplus \mathcal{L}^{\otimes 2} \oplus \ldots \oplus \mathcal{L}^{\otimes n-1}
$$

auf $Y$. 
Außerdem definiert die Multiplikation in $\mathcal{A}$ einen Bimodulhomomorphismus

$$
\varphi: \mathcal{L}^{\otimes n} \rightarrow \mathcal{O}_{Y}
$$

invertierbarer $\mathcal{O}_{Y}$-Bimoduln. Für diesen ist nach Konstruktion das folgende Diagramm

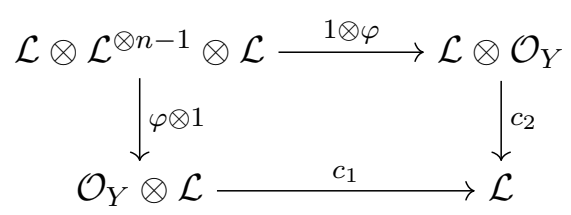

kommutativ. Dabei seien $c_{1}$ und $c_{2}$ die kanonischen Isomorphismen $\mathcal{O}_{Y} \otimes \mathcal{L} \cong \mathcal{L}$ und $\mathcal{L} \otimes \mathcal{O}_{Y} \cong \mathcal{L}$. Es gilt also $c_{2} \circ(1 \otimes \varphi)=c_{1} \circ(\varphi \otimes 1)$ oder kurz $1 \otimes \varphi=\varphi \otimes 1$.

\section{Konstruktion über einen Bimodul}

Auch in der Garbensituation können wir umgekehrt von einem $\mathcal{O}_{Y}$-Bimodul ausgehen und eine Garbe $\mathcal{A}$ auf $Y$ und über Push-forward damit auch auf $X-$ und dann sogar global - definieren. Diese Methode wurde wie erwähnt von Chan und Kulkarni eingeführt (siehe [Cha05, CK11]), und zwar in der folgenden Form für Garben:

Es sei $\mathcal{L}$ ein invertierbarer $\mathcal{O}_{Y}$-Bimodul. Die Rechtsstruktur sei dabei durch die mit $\sigma$ getwistete Linksstruktur gegeben. Invertierbar heiße für den Bimodul, dass $\mathcal{L}$ als $\mathcal{O}_{Y}$-Linksmodul invertierbar, also lokalfrei und von Rang 1 ist. Außerdem sei ein Homomorphismus

$$
\varphi: \mathcal{L}^{\otimes n} \rightarrow \mathcal{O}_{Y}
$$

gegeben, für den das Diagramm

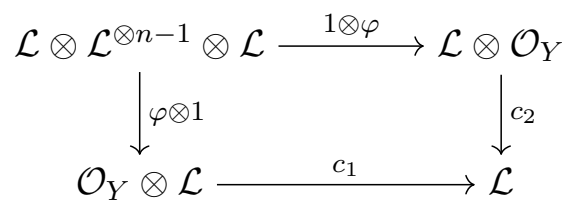

kommutiert; dies ist die von Chan und Kulkarni sogenannte Überschneidungsbedingung (englisch: overlap condition). Zu beachten ist, dass hier jeweils das Tensorprodukt in der Kategorie der Bimoduln zu bilden ist.

Mit Hilfe von $\mathcal{L}$ und $\varphi$ können wir nun eine Garbe definieren. Es sei

$$
\mathcal{A}=(Y \rightarrow X, \sigma, \mathcal{L}, \varphi)
$$

diejenige $\mathcal{O}_{X}$-Algebrengarbe, die als $\mathcal{O}_{Y}$-Bimodul durch

$$
\mathcal{A}=\mathcal{O}_{Y} \oplus \mathcal{L} \oplus \mathcal{L}^{\otimes 2} \oplus \ldots \oplus \mathcal{L}^{\otimes n-1}
$$


gegeben ist, versehen mit einer Multiplikation, die durch das Tensorprodukt $\mathcal{L}^{\otimes i} \times \mathcal{L}^{\otimes j} \rightarrow \mathcal{L}^{\otimes i+j}$ und durch den Morphismus $\varphi: \mathcal{L}^{\otimes n} \rightarrow \mathcal{O}_{Y}$ induziert wird.

Wir untersuchen hier nun, welche Beschreibung sich damit lokal ergibt:

Sei $U=\operatorname{Spec} R$ eine offene affine Teilmenge von $X$ und sei $U^{\prime}=p^{-1}(U)=$ Spec $R^{\prime}$ ihr ebenfalls affines Urbild in $Y$, dabei sei $U$ so klein gewählt, dass $R^{\prime}$ frei von Rang $n$ über $R$ ist. Es ist $R^{\prime}=\mathcal{O}_{Y}\left(p^{-1}(U)\right)$. Sei $u$ ein Erzeuger von $\mathcal{L}(U)$ als $R^{\prime}$-Modul, dann gilt

$$
\mathcal{A}(U)=R^{\prime} \oplus R^{\prime} u \oplus R^{\prime} u^{2} \oplus \ldots \oplus R^{\prime} u^{n-1}
$$

und $u x=\sigma(x) u$ für jedes $x \in R^{\prime}$. Sei außerdem $a=\varphi(u) \in R^{\prime}$. Dann gilt $u^{n}=\varphi(u)=a$. Folglich gilt auf der offenen affinen Menge $U=\operatorname{Spec}(R)$ :

$$
\mathcal{A}(U)=\left(R^{\prime} / R, \sigma, a\right) .
$$

Seien zwei offene affine Mengen $U_{1}=\operatorname{Spec} R_{1}$ und $U_{2}=\operatorname{Spec} R_{2}$ in $X$ mit ihren Urbildern $U_{1}^{\prime}=\operatorname{Spec} R_{1}^{\prime}$ und $U_{2}^{\prime}=\operatorname{Spec} R_{2}^{\prime}$ in $Y$ so gegeben, dass auch die Schnittmenge $U_{1} \cap U_{2}=$ Spec $R_{12}$ in $X$ und ihr Urbild $U_{1}^{\prime} \cap U_{2}^{\prime}=\operatorname{Spec} R_{12}^{\prime}$ in $Y$ affin sind und dass die jeweiligen Ringerweiterungen $R_{i}^{\prime} / R_{i}$ für $i=1,2$ und $R_{12}^{\prime} / R_{12}$ freie Moduln von Rang $n$ sind. Dann gilt

$$
R_{12}^{\prime} \otimes_{R_{1}^{\prime}}\left(R_{1}^{\prime} / R_{1}, \sigma, a_{1}\right) \cong R_{12}^{\prime} \otimes_{R_{2}^{\prime}}\left(R_{2}^{\prime} / R_{2}, \sigma, a_{2}\right)
$$

über $U_{1} \cap U_{2}$. Wir bezeichnen mit $u_{1}$ und $u_{2}$ die Unbestimmte in $\left(R_{1}^{\prime} / R_{1}, \sigma, a_{1}\right)$ bzw. in $\left(R_{2}^{\prime} / R_{2}, \sigma, a_{2}\right)$. Aufgrund der Charakterisierung der einzelnen Summanden in Lemma 2.4.3 folgt $R_{12}^{\prime} u_{1} \cong R_{12}^{\prime} u_{2}$. Folglich gibt es eine Einheit $\beta_{21} \in\left(R_{12}^{\prime}\right)^{\times}$mit $u_{2}=\beta_{21} u_{1}$. Insbesondere gilt damit dann auch $a_{2}=u_{2}^{n}=\mathrm{N}_{R_{12}^{\prime} / R_{12}}\left(\beta_{21}\right) u_{1}^{n}=\mathrm{N}_{R_{12}^{\prime} / R_{12}}\left(\beta_{21}\right) a_{1}$.

Ebenso folgt für drei offene affine Teilmengen $U_{1}, U_{2}, U_{3}$ von $X-$ mit den entsprechenden Bedingungen an ihre Urbilder und Schnittmengen -, dass dann $\beta_{13}=\beta_{12} \beta_{23}$ gilt.

Bemerkung 2.4.5. Ergänzen wir wie zuvor in der Notation des Bimoduls den erzeugenden Morphismus $\sigma$, schreiben also $\mathcal{L}_{\sigma}$, und ist $\mathcal{M}_{\tau}$ ein aus dem Linksmodul $\mathcal{M}$ mittels $\tau$ hervorgehender zweiter Bimodul, so ist auch hier das Tensorprodukt ein Bimodul und es gilt

$$
\mathcal{L}_{\sigma} \otimes \mathcal{M}_{\tau}=\left(\mathcal{L} \otimes \sigma^{*} \mathcal{M}\right)_{\sigma \tau} .
$$

Genau genommen bezeichnet $\mathcal{L}_{\sigma}$ eigentlich den Pull-back $\pi^{*} \mathcal{L}$ von $\mathcal{L}$ auf den Graphen $\Gamma \subset Y \times Y$ von $\sigma$, wobei $\pi$ die Projektion von $\Gamma$ auf die erste Komponente $Y$ sei, siehe etwa Artin und van den Bergh in [AVB90, Sec. 2] oder auch Chan in [Cha05, Sec. 2]. Chan empfiehlt, die obige Formel als Definition des Tensorproduktes zweier Bimoduln anzusehen. Ein formaler Beweis findet sich in [AVB90, Lemma (2.14)]. Insbesondere ist in unserem Fall

$$
\mathcal{L}_{\sigma}^{\otimes i}=\left(\mathcal{L} \otimes_{K^{\prime}} \sigma^{*} \mathcal{L} \otimes_{K^{\prime}} \ldots \otimes_{K^{\prime}} \sigma^{(i-1) *} \mathcal{L}\right)_{\sigma^{i}} .
$$


Bemerkung 2.4.6. Der Homomorphismus $\varphi: \mathcal{L}^{\otimes n} \rightarrow \mathcal{O}_{Y}$ induziert eine Isomorphie $\mathcal{L}^{\otimes n} \cong \mathcal{O}_{Y}\left(-D^{\prime}\right)$ mit einem effektiven Divisor $D^{\prime}$ auf $Y$. Dabei ist $\mathcal{O}_{Y}\left(-D^{\prime}\right)$ eine invertierbare Bimoduluntergarbe von $\mathcal{O}_{Y}$, die $\sigma$-invariant ist.

\subsubsection{Konkretes Beispiel: Doppelte Überlagerung}

In [CK03] klassifizieren Chan und Kulkarni die von ihnen so genannten DelPezzo-Ordnungen. Das sind Ordnungen, deren Duales der kanonischen Garbe ampel ist; eine nichtkommutative Verallgemeinerung von Del-Pezzo-Flächen. In [Cha05, Sec. 6] konstruiert Chan mit Hilfe des vorgestellten Cyclic-CoveringTricks Ordnungen von Grad 4 über der projektiven Ebene $\mathbb{P}^{2}$, die über einer glatten Quartik verzweigt sind. In [CK11] berechnen Chan und Kulkarni weitere Eigenschaften, zum Beispiel die Chern-Klassen, bevor sie zu ihrem eigentlichen Ziel, der Untersuchung von Modulräumen über Del-Pezzo-Ordnungen, übergehen. Außerdem stellen sie zu Beginn von [CK11, Sec.4] die Situation des Beispiels zusammengefasst vor: Sie starten mit einer doppelten Überlagerung $Y$ der projektiven Ebene, die über einer glatten Quartik verzweigt. Diese kann auch durch Blow-up des $\mathbb{P}^{2}$ in 7 Punkten in allgemeiner Lage erzeugt werden. Daher existieren in $Y$ zwei disjunkte exzeptionelle Kurven $E, E^{\prime}$, die über verschiedenen Bitangenten an die ebene Quartik liegen. Das Geradenbündel $L=\mathcal{O}_{Y}\left(E-E^{\prime}\right)$ ist dann ein 1-Kozykel und jeder Isomorphismus $L_{\sigma}^{\otimes 2} \cong \mathcal{O}_{Y}$ erfüllt die Überschneidungsbedingung. Folglich definiert die oben konstruierte Garbe $\mathcal{A}=\mathcal{O}_{Y} \oplus L_{\sigma}$ eine zyklische Algebra.

Dieses Beispiel wollen wir im Folgenden - ein wenig konkreter als Chan und Kulkarni - untersuchen:

Es sei $X=\mathbb{P}^{2}$ die projektive Ebene über einem algebraisch abgeschlossenen Körper $k$ von Charakteristik 0. Wir teilen $X$ in einen affinen Anteil $\mathbb{A}^{2}$ und eine unendlich ferne Gerade auf. Es ist dann $\mathbb{A}^{2}=\operatorname{Spec}(k[v, w])$ und für die Funktionenkörper gilt $k\left(\mathbb{A}^{2}\right)=k(X)=k(v, w)$.

Außerdem sei $C_{4} \subset X=\mathbb{P}^{2}$ eine glatte Quartik, deren affiner Anteil $C_{4} \cap \mathbb{A}^{2} \subset$ $\mathbb{A}^{2} \subset X$ als Nullstellenmenge eines irreduziblen Polynoms $f_{4} \in k[v, w]$ von Grad 4 gegeben ist. Die Homogenisierung von $f_{4}$ definiert dann die zugehörige projektive Kurve $C_{4}$ in $X=\mathbb{P}^{2}$.

\section{Konstruktion einer Überlagerung}

Unser Ziel ist es, eine Ordnung auf $X$ in einer Quaternionenalgebra über $k(X)$ zu konstruieren, die über dieser Quartik $C_{4}$ verzweigt. Dazu definieren wir zunächst eine zweiblättrige Überlagerung von $X$, die genau über $C_{4}$ verzweigt.

Es sei $K^{\prime}=k(X)\left[\sqrt{f_{4}}\right]$ die quadratische Körpererweiterung über $k(X)$ zum irreduziblen Polynom $T^{2}-f_{4} \in k(X)[T]$. Es sind dann $k(X) \rightarrow k(X)\left(\sqrt{f_{4}}\right)$ und $k[v, w] \rightarrow k[v, w]\left(\sqrt{f_{4}}\right)$ endliche Morphismen. 
Außerdem definiert $K^{\prime}$ in natürlicher Weise eine konstante $\mathcal{O}_{X}$-Algebrengarbe $\mathcal{A}^{\prime}$ auf $X$. In $\mathcal{A}^{\prime}$ können wir den ganzen Abschluss $\mathcal{A}$ der Strukturgarbe $\mathcal{O}_{X}$ bestimmen. Zu dieser Garbe $\mathcal{A}$ lässt sich in natürlicher Weise ein Schema $Y$ konstruieren und explizit berechnen, nämlich $Y=\operatorname{Spec}(\mathcal{A})$, der sogenannte ganze Abschluss des Schemas $X$ in $K^{\prime}$. Diese Konstruktion liefert ebenso einen zugehörigen endlichen Morphismus $p: Y \rightarrow X$ und für die Strukturgarbe $\mathcal{O}_{Y}$ von $Y$ gilt $p_{*} \mathcal{O}_{Y}=\mathcal{A}$. Siehe hierzu etwa Grothendiecks Ausführungen in EGA II ([Gro61, 6.3 Fermeture intégrale d'un préschéma]).

Dieser ganze Abschluss $Y$ von $X$ in $\mathcal{A}^{\prime}$ wird auch als Normalisierung von $X$ in der Körpererweiterung $K^{\prime} / k(X)$, bezeichnet, etwa von Mumford in [Mum99, III.8, Def. 3 und Thm. 3] oder [MO, Ch. V, Proposition-Definition 5.7].

Zum einen gilt in diesem Fall dann $k(Y)=K^{\prime}$. Zum anderen ist speziell über dem gewählten affinen Teil $\mathbb{A}^{2}=\operatorname{Spec}(k[v, w])$ das Urbild als $p^{-1}\left(\mathbb{A}^{2}\right)=$ $\operatorname{Spec}\left(k[v, w]\left[\sqrt{f_{4}}\right]\right)$ und die Einschränkung der Überlagerung $p: Y \rightarrow X$ auf $p^{-1}\left(\mathbb{A}^{2}\right) \rightarrow \mathbb{A}^{2}$ durch

$$
\operatorname{Spec}\left(k[v, w]\left[\sqrt{f_{4}}\right]\right) \rightarrow \operatorname{Spec}(k[v, w])
$$

und auf dem Niveau der Ringe durch die Einbettung

$$
k[v, w] \rightarrow k[v, w]\left(\sqrt{f_{4}}\right)
$$

gegeben.

Es ist $K^{\prime}=k(Y)$ eine galoissche Körpererweiterung von $k(X)$ von Grad 2 mit Galoisgruppe $\operatorname{Gal}(k(Y) / k(X)) \cong \mathbb{Z} / 2 \mathbb{Z}$. Sei $\sigma$ der erzeugende Automorphismus der Galoisgruppe. Da die obige Konstruktion funktoriell ist, operiert die Galoisgruppe $\operatorname{Gal}(k(Y) / k(X))=\{$ id, $\sigma\}$ kanonisch auf der gesamten quadratischen kommutativen Algebrengarbe $\mathcal{O}_{Y}$ über $\mathcal{O}_{X}$. Daher induziert $\sigma$ einen Automorphismus von $Y$ über $X$ von Ordnung 2, den wir ebenfalls mit $\sigma$ bezeichnen. Aus der Konstruktion folgt ebenfalls sofort, dass $\mathcal{O}_{Y}$ endlich als $\mathcal{O}_{X}$-Modulgarbe ist. Entsprechend ist das zugehörige Schema $p: Y \rightarrow X$ von Grad 2 über $X$. Weiter ist wegen der funktoriellen $\operatorname{Konstruktion} \operatorname{Aut}(Y / X) \cong \operatorname{Gal}(k(Y) / k(X))$ und es gilt $\left(\mathcal{O}_{Y}\right)^{\sigma}=\mathcal{O}_{X}$.

Letzteres gilt, da die projektive Ebene ein normales Schema ist und somit sämtliche Ringe $\mathcal{O}_{X}(U)$ ganzabgeschlossen in ihrem Quotientenkörper $k(X)$ sind. Da $\mathcal{O}_{Y}\left(p^{-1}(U)\right)$ gerade aus den über $\mathcal{O}_{X}(U)$ ganzen Elementen aus $k(Y)$ besteht, gilt somit $\mathcal{O}_{Y}\left(p^{-1}(U)\right) \cap k(X)=\mathcal{O}_{X}(U)$ und es folgt $\left(\mathcal{O}_{Y}\right)^{\sigma}=\mathcal{O}_{X}$.

\section{Verzweigungsverhalten der Überlagerung}

Im Folgenden untersuchen wir das Zerlegungsverhalten von $p: Y \rightarrow X$ über einem Punkt $x \in X^{(1)}$ der Kodimension 1. Da der lokale Ring $\mathcal{O}_{X, x}$ in diesem Fall ein diskreter Bewertungsring mit Funktionenkörper $k\left(\mathbb{P}^{2}\right)=k(v, w)$ ist, können wir die erforderlichen Resultate aus der Bewertungstheorie beziehen, 
siehe Abschnitt A.8.2 im Anhang. Das Primideal des lokalen Ringes $\mathcal{O}_{X, x}$ und die Bewertung werden durch $x$ gegeben und zumeist ebenfalls mit $x$ bezeichnet.

Ganz allgemein folgt aus den bewertungstheoretischen Überlegungen, dass sich die Urbilder der Kodimension-Eins-Punkte eines endlichen Morphismus aus den Fortsetzungen der Bewertungen ergeben:

Lemma 2.4.7. Sei $p: Y \rightarrow X$ ein endlicher Morphismus ganzer $k$-Schemata, dabei sei $Y$ der ganze Abschluss von $X$ im Funktionenkörper $k(Y)$. Zu einem Punkt $x \in X^{(1)}$ von Kodimension 1 seien $w_{1}, \ldots, w_{r}$ die Fortsetzungen auf $k(Y)$ der zu $x$ gehörigen diskreten Bewertung von $K$ und es seien $y_{1}, \ldots, y_{r}$ ihre Bewertungsideale. Dann gilt $p^{-1}(x)=\left\{y_{1}, \ldots, y_{r}\right\}$.

Beweis. Sei $\mathcal{A}$ der ganze Abschluss der Strukturgarbe $\mathcal{O}_{X}$ in $k(Y)$ und sei $v: k(X)^{\times} \rightarrow \mathbb{Z}$ die zu $x$ gehörige diskrete Bewertung auf dem Funktionenkörper $k(X)$ von $X$.

Sei $y \in Y$ ein Urbild von $x$. Ist $U \subset X$ eine offene affine Umgebung von $x$, so ist $p^{-1}(U) \subset Y$ eine offene affine Umgebung von $y$ und folglich ist $\mathcal{O}_{Y}\left(p^{-1}(U)\right)=$ $\mathcal{A}(U)$ eine ganze Ringerweiterung von $\mathcal{O}(U)$. Nach den Going-down-Theoremen ist damit auch $y \subset \mathcal{A}(U)$ ein Primideal von Höhe 1. Die Lokalisierung $\mathcal{A}(U)_{y}$ ist somit ein diskreter Bewertungsring mit maximalem Ideal $y \mathcal{A}(U)_{y}$. Da $y \cap \mathcal{O}_{X}(U)=x$ gilt, ist die zu $y$ gehörige Bewertung auf $k(Y)$ eine Fortsetzung von $v$.

Sei umgekehrt $w$ eine Bewertungsfortsetzung von $v$ auf $k(Y)$. Nach Konstruktion ist der Halm $\mathcal{A}_{x}$ der ganze Abschluss des lokalen Ringes $\mathcal{O}_{X, x}$ in $k(Y)$. Daher ist die Lokalisierung $\left(\mathcal{A}_{x}\right)_{\mathfrak{p}}$ nach dem Primideal $\mathfrak{p}=\left\{\alpha \in \mathcal{A}_{x} \mid w(\alpha)>0\right\}$ der Bewertungsring von $w$ in $k(Y)$ und $y:=\mathfrak{p}\left(\mathcal{A}_{x}\right)_{\mathfrak{p}}$ das Bewertungsideal (siehe etwa [Neu92, Kap. II, §8 Aufgabe 6]). Insbesondere enthält daher auch $\mathcal{A}(V) \subset\left(\mathcal{A}_{x}\right)_{\mathfrak{p}}$ für eine jede offene affine Umgebung $V$ von $x$ nur Elemente mit nichtnegativer Bewertung und $y \cap \mathcal{A}(V)=\{\alpha \in \mathcal{A}(V) \mid w(\alpha)>0\}$ ist ein Primideal in $\mathcal{A}(V)$ mit $(y \cap \mathcal{A}(V)) \cap \mathcal{O}_{X}(V)=x$. Folglich kann $y$ als Punkt in $\operatorname{Spec} \mathcal{A}(V) \subset Y$ aufgefasst werden und für ihn gilt $p(y)=x$.

Es sei nun $x \in X^{(1)}$ ein Punkt von Kodimension 1 in unserem gewählten Beispiel $X=\mathbb{P}^{2}$. Sein topologischer Abschluss $\bar{x}$ in $X$ ist eine irreduzible, reduzierte, projektive algebraische Kurve. Ohne Einschränkung können wir annehmen, dass $\bar{x}$ nicht die unendlich ferne Gerade ist. Dann ist auch $x$ selbst ein Punkt der affinen Ebene und dort ebenfalls von Kodimension 1, also gilt $x \in\left(\mathbb{A}^{2}\right)^{(1)}$, und die Kurve $\bar{x}$ ist in $\mathbb{A}^{2}=\operatorname{Spec}(k[v, w])$ als Nullstellenmenge eines irreduziblen Polynoms $g \in k[v, w]$ gegeben. Der lokale Ring in $x$ ist dann die Lokalisierung $\mathcal{O}_{X, x}=k[v, w]_{(g)}$, sein Quotientenkörper ist $\operatorname{Quot}\left(\mathcal{O}_{X, x}\right)=k(v, w)=k(X)$ und der Restklassenkörper ist der Quotientenkörper des Ringes $k[v, w] /(g)$, also $k(x)=\operatorname{Quot}(k[v, w] /(g))$.

Außerdem sei $\hat{\mathcal{O}}_{X, x}$ die Vervollständigung des diskreten Bewertungsringes mit zugehörigem Quotientenkörper $K_{x}=k(X)_{x}$, der Vervollständigung des Körpers $K=k(X)$ bezüglich der durch $x$ gegebenen diskreten Bewertung. 
Das Urbild $f^{-1}(x)$ in $Y$ von $x$ ist homöomorph zum topologischen Raum der Faser Spec $k(x) \times_{X} Y$. Für diese Faser und ihre Vervollständigung erhalten wir die beiden kommutativen Diagramme in Abbildung 2.1.
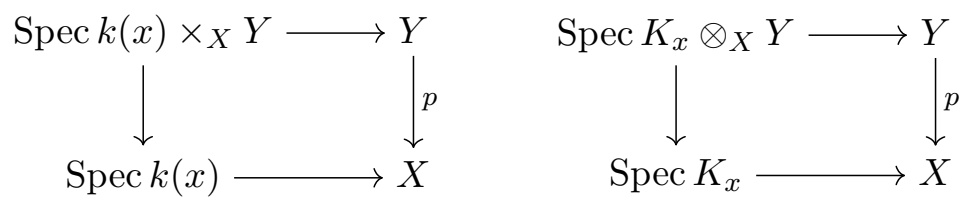

Abbildung 2.1: Faser eines Punktes $x$ von Kodimension 1

Für das Faserprodukt $\operatorname{Spec} \mathcal{O}_{X, x} \times_{X} Y$ des lokalen Ringes $\mathcal{O}_{X, x}$ in $x$ mit $Y$ und der entsprechenden Vervollständigung $\operatorname{Spec}\left(\hat{\mathcal{O}}_{X, x}\right) \times_{X} Y$ kommen die beiden kommutativen Diagramme in Abbildung 2.2 hinzu.
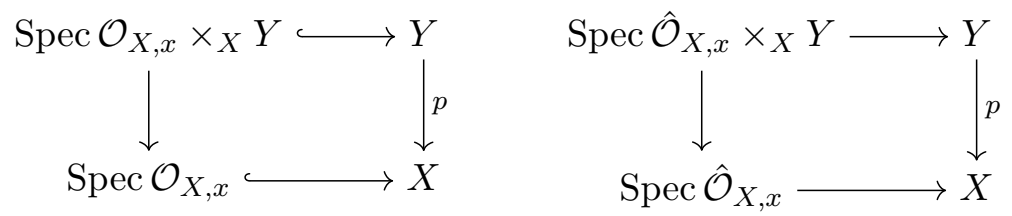

Abbildung 2.2: Faserprodukt eines Punktes $x$ von Kodimension 1

Da $k(Y)=k(X)[T] /\left(T^{2}-f_{4}\right)$ der Funktionenkörper unserer Überlagerung $p: Y \rightarrow X$ ist, untersuchen wir nun das Zerlegungsverhalten des in $k(X)[T]$ irreduziblen Polynoms $T^{2}-f_{4}$ über der Vervollständigung $K_{x}=k(X)_{x}$ :

Erster Fall: Es ist $\overline{f_{4}}$ kein Quadrat in $k(x)$.

Dann ist $T^{2}-\overline{f_{4}}$ irreduzibel über $k(x)$ und $T^{2}-f_{4}$ ist irreduzibel über $K_{x}$. Für Verzweigungsindex und Trägheitsgrad der Erweiterung $K^{\prime} / K$ und für die Anzahl $r$ der irreduziblen Komponenten von $T^{2}-\overline{f_{4}}$ über $K_{x}$ gilt damit $r=1$, $e=1$ und $f=2$. Folglich ist $k(X)_{x} \otimes_{k(X)} k(Y)=k(X)_{x}[T] /\left(T^{2}-f_{4}\right)$ eine quadratische Körpererweiterung von $k(X)_{x}$ und wir erhalten das kommutative Diagramm:

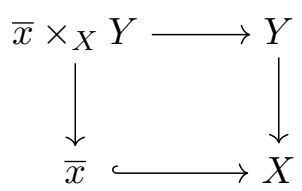

Dann ist $\left(\bar{x} \times_{X} Y\right)$ eine irreduzible Kurve von Grad 2, also eine zweiblättrige Überlagerung über der Kurve $\bar{x}$. Der generische Punkt $y$ dieser irreduziblen Kurve ist der eindeutige Punkt über $x \in X^{(1)}$, es gilt also $p^{-1}(x)=\{y\}$. 
Zweiter und dritter Fall: Es ist $\overline{f_{4}}$ ein Quadrat in $k(x)$.

Dann lässt sich das Polynom $T^{2}-\overline{f_{4}}$ über dem Restklassenkörper in zwei Linearfaktoren $T^{2}-\overline{f_{4}}=\left(T-\alpha_{1}\right)\left(T-\alpha_{2}\right)$ mit $\alpha_{1}, \alpha_{2} \in k(x)$ zerlegen. Je nachdem, ob $\alpha_{1}=\alpha_{2}$ ist oder nicht, ergibt sich das folgende Verhalten:

Zweiter Fall: Es gilt $\alpha_{1}=\alpha_{2}$ und also $T^{2}-\overline{f_{4}}=(T-\alpha)^{2}$ mit einem $\alpha \in k(x)$.

Ein Koeffizientenvergleich liefert $\alpha=0$ und damit auch $\overline{f_{4}}=-\alpha^{2}=0$ in $k(x)=k[v, w] /(g)$. Es folgt zuerst $g \mid f_{4}$ in $k[v, w][T]$ und dann, da $f_{4}$ irreduzibel ist, auch $g=f_{4}$ (wir gehen davon aus, dass beide Polynome normiert sind). Daher ist $\bar{x}=C_{4}$, die glatte Quartik. Außerdem ist in diesem Fall nach dem Eisenstein-Kriterium das Polynom $T^{2}-f_{4}$ irreduzibel über $k(X)_{x}[T]$ und es gilt $r=1, e=2, f=1$. Das bedeutet, dass über $\bar{x}=C_{4}$ genau eine reduzierte Kurve in $Y$ liegt, und es gilt

$$
\begin{aligned}
\hat{\mathcal{O}}_{X, x} \otimes_{\mathcal{O}_{X, x}} k(Y) & =k(x)\left[\left[f_{4}\right]\right] \otimes_{\mathcal{O}_{X, x}} k(Y) \\
& \cong\left(k(x)\left[\left[f_{4}\right]\right]\right)[T] /\left(T^{2}-f_{4}\right) \\
& \cong k(x)\left[\left[\sqrt{f_{4}}\right]\right] .
\end{aligned}
$$

Dritter Fall: Es gilt $\alpha_{1} \neq \alpha_{2} \in k(x)$ und $T^{2}-\overline{f_{4}}=\left(T-\alpha_{1}\right)\left(T-\alpha_{2}\right)$.

Nach dem Henselschen Lemma (siehe etwa Lemma A.8.1 im Anhang) ist dann auch $T^{2}-f_{4}$ über $k(X)_{x}$ reduzibel. Es ist dann $r=2, e:=e_{1}=e_{2}=1$ und $f:=f_{1}=f_{2}=1$. Damit und aus dem Chinesischen Restsatz ergeben sich die Zerlegungen

$$
k(X)_{x} \otimes_{k(X)} k(Y) \cong k(X)_{x} \oplus k(X)_{x}
$$

und

$$
\hat{\mathcal{O}}_{X, x} \otimes_{\mathcal{O}_{X, x}} k(Y) \cong \hat{\mathcal{O}}_{X, x} \oplus \hat{\mathcal{O}}_{X, x} .
$$

Über $x$ liegen dann genau zwei Punkte $y_{1}, y_{2} \in Y$. Falls $\bar{x}$ eine glatte Kurve beschreibt, so erfüllen die beiden Kurven $\overline{y_{i}}$ die Isomorphien

$$
\left.p\right|_{\overline{y_{i}}}: \overline{y_{i}} \stackrel{\cong}{\longrightarrow} \bar{x}
$$

für $i=1,2$.

Bemerkung 2.4.8. Insgesamt folgt aus all diesen Fällen, dass $Y$ genau über der durch $f_{4}$ definierten Quartik $C_{4}$ verzweigt ist.

\section{Konstruktion eines Geradenbündels und einer Algebrengarbe $\mathcal{A}$ mit Hilfe von Doppeltangenten}

Sei jetzt $x \in X^{(1)}$ ein solcher Punkt der Kodimension 1, dass seine Kurve $\bar{x} \subset \mathbb{P}^{2}$ eine Gerade ist. Dann ist $\left(\bar{x} \cap C_{4}\right)$ ein 0-dimensionales Schema von Länge 4. Die obige Fallunterscheidung beweist den folgenden Satz: 
Satz 2.4.9. Sei $x \in X^{(1)}$ der generische Punkt einer Geraden $\ell=\overline{\{x\}} \subset X$. Dann ist $\overline{f_{4}}$ genau dann ein Quadrat in $k(x)$, wenn $\ell$ eine Doppeltangente an die Quartik $C_{4}$ ist. In diesem Fall ist $e=f=1, r=2$ und über $\ell$ liegen zwei Kurven $\overline{\left\{y_{1}\right\}}, \overline{\left\{y_{2}\right\}} \subset Y$. Für diese gilt dann $\overline{\left\{y_{i}\right\}} \cong \ell \cong \mathbb{P}^{1}$.

An eine Quartik über einem algebraisch abgeschlossenen Körper existieren stets 28 Doppeltangenten (siehe etwa [Har77, Ch.IV, Ex. 2.3(h)]). Für das Urbild einer solchen Doppeltangente $\ell$ gilt jeweils $p^{-1}(\ell)=\left\{\overline{\left\{y_{1}\right\}}, \overline{\left\{y_{2}\right\}}\right\}$ mit $y_{1} \neq y_{2}$. Daher gibt es insgesamt 56 Kurven in $Y$, die auf diese Weise entstehen; und jede ist isomorph zum $\mathbb{P}^{1}$. Für den Automorphismus $\sigma$ von $Y / X$ gilt insbesondere $\sigma\left(y_{1}\right)=y_{2}$.

Seien nun $\ell \neq \ell^{\prime}$ zwei Doppeltangenten an die Quartik $C_{4}$ in $X$ und seien $E=\overline{y_{1}}$ und $\sigma(E)=\overline{y_{2}}$ bzw. $E^{\prime}=\overline{y_{1}^{\prime}}$ und $\sigma\left(E^{\prime}\right)=\overline{y_{2}^{\prime}}$ jeweils ihre beiden Urbilder unter der Überlagerung $p: Y \rightarrow X$.

Wir betrachten $\left(\ell-\ell^{\prime}\right)$ als Hauptdivisor auf $X$. Für ihn gilt $\mathcal{O}_{X}\left(\ell-\ell^{\prime}\right)=\mathcal{O}_{X}$, und auf $Y$ ergibt sich

$$
p^{*}\left(\ell-\ell^{\prime}\right)=(E+\sigma(E))-\left(E^{\prime}+\sigma\left(E^{\prime}\right)\right) .
$$

Damit erhalten wir für den Divisor $D=\left(E-E^{\prime}\right)$ auf $Y$ die Isomorphie

$$
\begin{aligned}
\mathcal{O}_{Y}(D) \otimes \sigma^{*} \mathcal{O}_{Y}(D) & \cong \mathcal{O}_{Y}\left(E-E^{\prime}\right) \otimes \mathcal{O}_{Y}\left(\sigma(E)-\sigma\left(E^{\prime}\right)\right) \\
& =\mathcal{O}_{Y}\left((E+\sigma(E))-\left(E^{\prime}+\sigma\left(E^{\prime}\right)\right)\right) \\
& \cong p^{*}\left(\mathcal{O}_{X}\left(\ell-\ell^{\prime}\right)\right) \cong p^{*} \mathcal{O}_{X}=\mathcal{O}_{Y} .
\end{aligned}
$$

Für das durch diesen Divisor auf $Y$ definierte Geradenbündel

$$
\mathcal{L}=\mathcal{O}_{Y}(D)=\mathcal{O}_{Y}\left(E-E^{\prime}\right)
$$

ergibt die Verknüpfung der obigen Isomorphismen somit einen Isomorphismus

$$
\varphi: \mathcal{L} \otimes \sigma^{*} \mathcal{L} \rightarrow \mathcal{O}_{Y}
$$

Dieser erfüllt die Überschneidungsbedingung (overlap condition) $\varphi \otimes 1=1 \otimes \varphi$.

Wie im Unterabschnitt „Konstruktion über einen Bimodul“ von 2.4.3 (Seite 40) beschrieben, können wir mit Hilfe von $\mathcal{L}$ daher die zyklische Algebrengarbe

$$
\mathcal{A}=(Y \rightarrow X, \sigma, \mathcal{L}, \varphi)=\mathcal{O}_{Y} \oplus \mathcal{L}
$$

definieren. Die Multiplikation ist dabei durch das Tensorprodukt von Bimoduln $\mathcal{L}^{\otimes i} \times \mathcal{L}^{\otimes j} \rightarrow \mathcal{L}^{\otimes i+j}$ für $i, j=0,1$ und durch den Morphismus $\varphi$ induziert. 


\section{Die Algebrengarbe im generischen Punkt}

Sei $\eta$ der generische Punkt von $Y$. Wir wollen klären, wie für die soeben konstruierte Algebra $\mathcal{A}$ der Halm im generischen Punkt $\mathcal{A}_{\eta}=\mathcal{O}_{Y, \eta} \oplus \mathcal{L}_{\eta}=$ $k(Y) \oplus \mathcal{L}_{\eta}$ über $k(X)=k\left(\mathbb{P}^{2}\right)$ aussieht. Um diese Frage zu beantworten, bestimmen wir die Isomorphie

$$
\varphi: \mathcal{L} \otimes \sigma^{*}(\mathcal{L}) \stackrel{\cong}{\longrightarrow} p^{*}\left(\mathcal{O}_{X}\left(\ell-\ell^{\prime}\right)\right) \stackrel{\cong}{\longrightarrow} p^{*} \mathcal{O}_{X}=\mathcal{O}_{Y}
$$

möglichst explizit:

Es seien $f_{\ell}$ und $f_{\ell^{\prime}} \in k[v, w] \subset k(X)$ Polynome, die die gewählten Doppeltangenten $\ell$ und $\ell^{\prime}$ in $X$ definieren, und es sei $t=f_{\ell} / f_{\ell^{\prime}}$ deren Quotient in $k(X)$. Für diese rationale Funktion $t$ gilt dann $\left.t\right|_{\ell}=0$ und $\left.t\right|_{\ell^{\prime}}=\infty$, genauer gesagt hat $t$ entlang von $\ell$ eine Nullstelle und entlang von $\ell^{\prime}$ eine Polstelle von jeweils einfacher Ordnung. Außerdem erzeugt $t$ den Divisor

$$
(t)=\ell-\ell^{\prime},
$$

weshalb insbesondere $\mathcal{O}_{X}((t))=\mathcal{O}_{X}\left(\ell-\ell^{\prime}\right)$ gilt. Die erforderliche Isomorphie

$$
p^{*}\left(\mathcal{O}_{X}\left(\ell-\ell^{\prime}\right)\right) \stackrel{\cong}{\longrightarrow} p^{*} \mathcal{O}_{X}=\mathcal{O}_{Y}
$$

können wir folglich durch Pull-back der Isomorphie

$$
\begin{gathered}
\mathcal{O}_{X}((t))=\mathcal{O}_{X}\left(\ell-\ell^{\prime}\right) \longrightarrow \mathcal{O}_{X} \\
t^{-1}=\frac{f_{\ell^{\prime}}}{f_{\ell}} \longmapsto 1
\end{gathered}
$$

(beziehungsweise $1 \mapsto t$ ) realisieren.

Die Algebra $\mathcal{A}_{\eta}$ im generischen Punkt können wir somit durch den $k(Y)$ Linksmodul

$$
\mathcal{A}_{\eta}=k(Y) \oplus k(Y) \cdot u
$$

mit einer Unbestimmten $u$ beschreiben, zusammen mit der Multiplikation, die durch die beiden Regeln

$$
u \alpha=\sigma(\alpha) u \quad \text { und } \quad u^{2}=\frac{f_{\ell}}{f_{\ell^{\prime}}}
$$

für $\alpha \in k(Y)$ gegeben wird. Sie ist also in der Tat eine zyklische Algebra über dem Funktionenkörper $k(X)$. Da sie von Dimension 4 über $k(X)$ ist, ist $\mathcal{A}_{\eta}$ somit eine Quaternionenalgebra über $k(X)$. 


\section{Verzweigungsverhalten der Algebra}

Korollar 4.4 in [Cha05] besagt, dass die Azumaya-Algebra $\mathcal{A}_{\eta}$ genau über der Quartik $C_{4}$ verzweigt ist. Dieses können wir auch konkret in den lokalen Ringen nachrechnen:

Sei $x \in X^{(1)}$ ein Punkt von Kodimension 1. Nach Satz 2.3.13 ist $\mathcal{A}_{\eta}$ genau dann über den Bewertungsring $\mathcal{O}_{X, x}$ fortsetzbar, wenn ihre Brauerklasse $\left[\mathcal{A}_{\eta}\right]$ im Kern der Verzweigungsabbildung $r_{x}: \operatorname{Br}(k(X)) \rightarrow X(k(x))$ liegt. Sei $x$ zunächst solch ein Punkt, dass die Restklasse von $f_{4}$ kein Quadrat im Restklassenkörper $k(x)$ ist. Insbesondere ist $x$ dann keiner der Punkte $\left(f_{4}\right),\left(f_{\ell}\right),\left(f_{\ell^{\prime}}\right)$, und nach den vorhergehenden Rechnungen ist $k(Y)$ unverzweigt über $k(X)$. Wir können das Bild von $\mathcal{A}_{\eta}=k(Y) \oplus k(Y) u$ unter der Verzweigungsabbildung daher wie folgt bestimmen: Für die beiden Elemente $u_{\text {id }}=1$ und $u_{\sigma}=u$ aus $\mathcal{A}_{\eta}^{\times}$gilt

$$
\operatorname{id}(\alpha)=1 \alpha 1^{-1}, \quad \sigma(\alpha)=u \alpha u^{-1}
$$

für alle $\alpha \in k(Y)$. Infolgedessen repräsentiert der Kozykel $f: G \times G \rightarrow k(Y)^{\times}$ mit $f(\rho, \tau)=u_{\rho} u_{\tau} u_{\rho \tau}^{-1}$ bzw. mit

$$
f\left(\sigma^{i}, \sigma^{j}\right)= \begin{cases}1, & \text { falls } i \equiv 0 \text { oder } j \equiv 0 \bmod 2 \\ u^{2}=\frac{f_{\ell}}{f_{\ell^{\prime}}}, & \text { falls } i \equiv j \equiv 1 \bmod 2\end{cases}
$$

die Brauerklasse $\left[\mathcal{A}_{\eta}\right]$ in $\mathrm{H}^{2}\left(G, k(Y)^{\times}\right)$. Die Fortsetzung $\nu_{x}: k(Y)^{\times} \rightarrow \mathbb{Z}$ der zu $x$ gehörigen diskreten Bewertung von $k(X)$ liefert den trivialen Kozykel

$$
f^{\prime}\left(\sigma^{i}, \sigma^{j}\right)= \begin{cases}\nu_{x}(1)=0, & \text { falls } i \equiv 0 \text { oder } j \equiv 0 \bmod 2 \\ \nu_{x}\left(\frac{f_{\ell}}{f_{\ell^{\prime}}}\right)=0, & \text { falls } i \equiv j \equiv 1 \bmod 2\end{cases}
$$

in $\mathcal{Z}^{2}(G, \mathbb{Z})$. Dessen Bild in der Charaktergruppe, also $r_{x}\left(\mathcal{A}_{\eta}\right)$, ist somit ebenfalls trivial. Folglich ist $\mathcal{A}_{\eta}$ unverzweigt über $x$.

Ist hingegen die Restklasse von $f_{4}$ ein Quadrat in $k(x)$, so ist $k(Y)$ verzweigt über $k(X)$ und das Bild unter $r_{x}$ kann nicht wie soeben bestimmt werden.

Es ist $\mathcal{A}_{\eta}$ gerade die Symbolalgebra $\left(f_{4}, f_{\ell} / f_{\ell^{\prime}}, \zeta_{2}\right)$ über $k(X)$ mit den beiden Erzeugern $\sqrt{f_{4}}$ und $u$ mit Quadraten ${\sqrt{f_{4}}}^{2}=f_{4}$ und $u^{2}=f_{\ell} / f_{\ell^{\prime}}$ und mit der Vertauschungsregel $u \sqrt{f_{4}}=\zeta_{2} \sqrt{f_{4}} u=-\sqrt{f_{4}} u$. Nach Satz 2.3.18 ist $\mathcal{A}_{\eta}=$ $\left(f_{4}, f_{\ell} / f_{\ell^{\prime}}, \zeta_{2}\right)$ genau dann über den Bewertungsring $\mathcal{O}_{X, x}$ fortsetzbar, wenn das zahme Symbol $\partial_{x}\left(f_{4}, f_{\ell} / f_{\ell^{\prime}}\right)$ ein Quadrat in $k(x)^{\times}$ist.

Sei $\nu_{x}: k(X)^{\times} \rightarrow \mathbb{Z}$ die zu $x$ gehörige diskrete Bewertung. Ist $x$ ein Punkt in $X$ von Kodimension 1 ungleich $f_{4}, f_{\ell}, f_{\ell^{\prime}}$, so gilt $\nu_{x}\left(f_{4}\right)=\nu_{x}\left(f_{\ell}\right)=\nu_{x}\left(f_{\ell^{\prime}}\right)=0$ und es ist

$$
\partial_{x}\left(f_{4}, \frac{f_{\ell}}{f_{\ell^{\prime}}}\right)=(-1)^{0 \cdot 0} \overline{\left(\frac{f_{4}^{0}}{\left(\frac{f_{\ell}}{f_{\ell^{\prime}}}\right)^{0}}\right)}=1
$$


ein Quadrat. Somit ist $\mathcal{A}_{\eta}$ über den Punkten $x \neq f_{4}, f_{\ell}, f_{\ell^{\prime}}$ von Kodimension 1 unverzweigt.

Außerdem gilt

$$
\partial_{f_{\ell}}\left(f_{4}, \frac{f_{\ell}}{f_{\ell^{\prime}}}\right)=\overline{f_{4}}, \quad \partial_{f_{\ell^{\prime}}}\left(f_{4}, \frac{f_{\ell}}{f_{\ell^{\prime}}}\right)={\overline{f_{4}}}^{-1}, \quad \partial_{f_{4}}\left(f_{4}, \frac{f_{\ell}}{f_{\ell^{\prime}}}\right)=\overline{\left(\frac{f_{\ell^{\prime}}}{f_{\ell}}\right)} .
$$

Da $\ell$ und $\ell^{\prime}$ Doppeltangenten an die Quartik $C_{4}$ sind, sind die Restklassen von $f_{4}$ und $f_{4}^{-1}$ Quadrate in $k\left(f_{\ell}\right)$ bzw. $k\left(f_{\ell^{\prime}}\right)$. Somit ist $\mathcal{A}_{\eta}$ auch über den beiden beiden Punkten $f_{\ell}$ und $f_{\ell^{\prime}}$ unverzweigt.

Als Produkt zweier verschiedener Geraden, also Polynomen von Grad 1, ist $f_{\ell^{\prime}} f_{\ell}$ kein Quadrat in $k\left(f_{4}\right)$. Daher sind $\mathcal{A}_{\eta}$ und auch die Algebrengarbe $\mathcal{A}$ über der von $f_{4}$ definierten Quartik $C_{4}$ verzweigt. 


\section{Kapitel 3}

\section{Kompositionsalgebren}

Allgemeiner als in den bislang betrachteten (assoziativen) Algebren wollen wir in dem zweiten Teil dieser Arbeit, das heißt in den Kapiteln 3 bis 5, auch Multiplikationen erlauben, die nicht unbedingt assoziativ sein müssen. Dabei wollen wir uns auf spezielle nichtassoziative - oder genauer: nicht notwendig assoziative - Algebren, die sogenannten Kompositionsalgebren, beschränken. Diese zeichnen sich dadurch aus, dass sie neben der AlgebrenStruktur - Addition, Skalarmultiplikation und Multiplikation - eine mit der Multiplikation verträgliche nichtausgeartete quadratische Form, die sogenannte Norm, besitzen. Man kann diese Kompositionsalgebren als Verallgemeinerung von Quaternionenalgebren verstehen. Es stellt sich daher die Frage, ob man auch Garben von Kompositionsalgebren auf geeigneten Schemata betrachten kann.

In den ersten beiden Abschnitten dieses Kapitels wiederholen wir die Definition und ausgewählte Eigenschaften nichtassoziativer Algebren und quadratischer Formen. Im dritten Abschnitt werden Kompositionsalgebren über Ringen definiert und die grundlegenden Eigenschaften dargestellt. Dabei folgen wir im Wesentlichen den Ausführungen über Kompositionsalgebren über Körpern von Springer und Veldkamp in [SV00, Ch. 1] und untersuchen, wie wir die Aussagen für Algebren über Ringen verallgemeinern können. Die Definition von Kompositionsalgebren über Ringen ist von Knus aus [Knu91, V (7.1)] - mit seiner Einschränkung auf nichtausgeartete Formen - übernommen. Im vierten Abschnitt folgen einige zusätzliche Untersuchungen. In Kapitel 4 wird ein nichtassoziatives Analogon zu den Maximalordnungen in Algebren eingeführt und untersucht; Maximalordnungen in Oktavenalgebren über vollständigen diskreten Bewertungsringen und über $\mathbb{Z}$ wurden bereits von van der Blij und Springer in [vBS59] betrachtet. In Kapitel 5 zeigen wir, wie man tatsächlich Garben von Kompositionsalgebren und Garben von Maximalordnungen definieren kann. Abschließend konstruieren und untersuchen wir im letzten Abschnitt ein konkretes nichttriviales Beispiel einer solchen Kompositionsalgebrengarbe auf einem projektiven dreidimensionalen Schema. 
Im gesamten Kapitel bezeichne $R$ einen kommutativen Ring mit Eins.

\subsection{Nichtassoziative Algebren}

Wir nutzen die Definition nichtassoziativer Algebren von Lang in [Lan02]:

Definition. Eine nicht notwendig assoziative Algebra über einem kommutativen Ring $R$ ist ein $R$-Modul $A$ zusammen mit einer $R$-bilinearen Abbildung $m: A \times A \rightarrow A$.

Die Abbildung $m$ ergibt die gewünschte nicht notwendig assoziative Multiplikation $x y=m(x, y)$ für $x, y \in A$. Diese erfüllt dann neben der Distributivität noch eine gewisse $R$-Assoziativität, nämlich $(\lambda x) y=\lambda(x y)=x(\lambda y)$ für alle $x, y \in A, \lambda \in R$.

Besitzt die Algebra $A$ ein Einselement $e$, so definiert - ähnlich zum assoziativen Fall - die Abbildung $\varphi: R \rightarrow A, \lambda \mapsto \lambda e$ einen Ringhomomorphismus (nicht notwendig assoziativer Ringe), dessen Bild dann automatisch im Zentrum $Z(A)$ von $A$ liegt. Auch hier gilt, dass die Abbildung $\varphi$ genau dann injektiv ist, wenn $A$ als $R$-Modul treu ist, d.h. genau dann, wenn der Annullator $\operatorname{Ann}(A)=\{\lambda \in R \mid \lambda x=0 \forall x \in A\}$ trivial ist.

Übrigens bildet das Zentrum von $A$ zwar stets einen $R$-Untermodul von $A$, muss im Allgemeinen aber kein Unterring sein.

\subsubsection{Skalarerweiterung}

Ist $A$ eine nicht notwendig assoziative $R$-Algebra und $S$ eine kommutative (und assoziative) $R$-Algebra, so ist das Tensorprodukt $S \otimes_{R} A$ von $R$-Moduln mit der durch

$$
(\lambda \otimes x) \cdot(\mu \otimes y)=(\lambda \mu) \otimes(x y)
$$

für $\lambda, \mu \in S, x, y \in A$ definierten Multiplikation eine nicht notwendig assoziative $S$-Algebra.

\subsection{Quadratische Formen}

Wir folgen in der Definition der quadratischen Formen und insbesondere in der Unterscheidung zwischen nichtausgearteten und regulären quadratischen Moduln der Einführung von Kneser in [Kne02]. In der englischen Literatur wird teilweise sogar zwischen nondegenerated (nichtausgeartet), nonsingular (die nachfolgende Abbildung $b_{M}$ ist bijektiv) und regular (regulär) unterschieden. In der genauen Ausprägung von nichtausgearteten quadratischen Formen folgen wir ebenfalls Kneser sowie Knus ([Knu91, V (7.1) bzw. I (3.2)]), siehe hierzu genauer Abschnitt 3.2.2. 
Definition. Eine quadratische Form auf einem $R$-Modul $M$ ist eine Abbildung $q: M \rightarrow R$, die

$$
q(\lambda x)=\lambda^{2} q(x)
$$

für alle $\lambda \in R, x \in M$ erfüllt und für die $b: M \times M \rightarrow R$,

$$
b(x, y):=q(x+y)-q(x)-q(y)
$$

eine symmetrische Bilinearform definiert. Ein Modul $M$ mit quadratischer Form $q$ wird als quadratischer Modul $(M, q)$ bezeichnet.

Definition. Ein quadratischer Modul $(M, q)$, die quadratische Form $q$ bzw. die Bilinearform $b$ heißt nichtausgeartet, wenn die Abbildung

$$
b_{M}: M \longrightarrow M^{*}=\operatorname{Hom}_{R}(M, R), x \longmapsto(y \mapsto b(x, y))
$$

injektiv ist. Der Modul $(M, q)$ heißt regulär, wenn die Abbildung $b_{M}$ bijektiv ist und wenn $M$ als Modul endlich erzeugt und projektiv ist.

Einen Untermodul $B$ eines quadratischen Moduls $(M, q)$ nennen wir nichtausgeartet bzw. regulär, wenn $B$ bezüglich der Einschränkung der Bilinearform nichtausgeartet bzw. regulär ist.

Für einen endlich-dimensionalen Vektorraum über einem Körper fallen die beiden Fälle nichtausgeartet und regulär zusammen.

Ist $(M, b)$ ein freier Modul mit Basis $e_{1}, \ldots, e_{n}$, so lässt sich die Bilinearform durch die Gramsche Matrix $B=\left(b\left(e_{i}, e_{j}\right)\right)_{i, j=1, \ldots, n}$ beschreiben. Ihre Determinante $d\left(e_{1}, \ldots, e_{n}\right)=\operatorname{det}(B)$ ist bis auf ein Quadrat aus der Einheitengruppe $R^{\times}$unabhängig von der Wahl der Basis eindeutig durch $(M, b)$ bestimmt. Die Restklasse $\operatorname{det}(M)=d\left(e_{1}, \ldots, e_{n}\right)\left(R^{\times}\right)^{2}$ heißt Determinante von $(M, b)$. Diese Determinante normiert man manchmal noch um ein gewisses Vorzeichen. Es sei $d(M)=(-1)^{m} \operatorname{det}(M)$ die Diskriminante von $(M, b)$, wobei $n=2 m$ oder $n=2 m+1$ gelte.

Satz 3.2.1 ([Kne02, (1.15) Satz]). Ein freier Modul $(M, b)$ mit Basis $e_{1}, \ldots, e_{n}$ über $R$ ist genau dann nichtausgeartet, wenn $d\left(e_{1}, \ldots, e_{n}\right)$ kein Nullteiler in $R$ ist. Er ist genau dann regulär, wenn $d\left(e_{1}, \ldots, e_{n}\right)$ in $R$ invertierbar ist.

Ist $(M, b)$ ein projektiver Modul von konstantem Rang $n$, so sei das Diskriminantenideal $d(M, b)$ dasjenige Ideal, das von allen Elementen $d\left(x_{1}, \ldots, x_{n}\right)=$ $\operatorname{det}\left(\left(b\left(x_{i}, x_{j}\right)\right)_{i, j=1, \ldots, n}\right)$ mit $x_{1}, \ldots, x_{n} \in M$ erzeugt wird.

Korollar 3.2.2 ([Knu91, I (3.2.1) Example]). Ein projektiver $R$-Modul $(M, b)$ von konstantem Rang $n$ ist genau dann regulär, wenn $d(M, b)=R$ ist.

Wir bezeichnen mit $B^{\perp}=\{x \in M \mid b(x, z)=0 \forall z \in B\}$ den orthogonalen Untermodul einer Teilmenge $B \subseteq M$. Ist $B$ ein Untermodul von $M$, so ist $B^{\perp}$ der Kern der Abbildung $b_{B}^{\prime}: M \rightarrow B^{*}=\operatorname{Hom}_{R}(B, R), x \longmapsto(y \mapsto b(x, y))$. 
Es gilt genau dann $B \cap B^{\perp}=\{0\}$, wenn $B$ nichtausgeartet ist. Es gilt genau dann $M=B \oplus B^{\perp}$, wenn $b_{B}^{\prime}$ eine Bijektion von $B$ auf $b_{B}^{\prime}(M)$ induziert.

Satz 3.2.3 ([Kne02, nach (1.5) Satz, (1.6) Satz]). Jeder reguläre Untermodul eines quadratischen Moduls spaltet als orthogonaler Summand ab.

Eine orthogonale Summe quadratischer Moduln ist genau dann nichtausgeartet bzw. regulär, wenn dies für jeden einzelnen Summanden gilt.

\subsubsection{Skalarerweiterung}

Sind $S$ eine kommutative $R$-Algebra und $(M, q)$ ein quadratischer $R$-Modul mit zugehöriger Bilinearform $\langle\cdot, \cdot\rangle$, so definieren

$$
\begin{aligned}
q_{S}(\lambda \otimes x) & =\lambda^{2} q(x), \\
\langle\lambda \otimes x, \mu \otimes y\rangle_{S} & =\lambda \mu\langle x, y\rangle
\end{aligned}
$$

für $\lambda, \mu \in S, x, y \in M$ eine quadratische Form und ihre zugehörige Bilinearform auf dem $S$-Modul $S \otimes M$. Die quadratische Form auf einem allgemeinen Element sieht dann wie folgt aus:

$$
q_{S}\left(\sum_{i} \lambda_{i} \otimes x_{i}\right)=\sum_{i} \lambda_{i}^{2} q\left(x_{i}\right)+\sum_{i<j} \lambda_{i} \lambda_{j}\left\langle x_{i}, x_{j}\right\rangle
$$

für $\lambda_{i} \in S, x_{i} \in M$.

Lemma 3.2.4. Seien $(M, q)$ ein quadratischer $R$-Modul und $S$ eine kommutative $R$-Algebra. Ist $M$ endlich erzeugt und projektiv, so entspricht die Abbildung

$$
\begin{aligned}
b_{S \otimes C}: S \otimes C & \longrightarrow S^{*}=\operatorname{Hom}_{S}(S \otimes C, S) \\
x^{\prime} & \longmapsto \quad\left(y^{\prime} \mapsto\left\langle x^{\prime}, y^{\prime}\right\rangle_{S}\right)
\end{aligned}
$$

der durch Tensorieren mit $S$ aus $b_{C}: C \rightarrow \operatorname{Hom}_{R}(C, R), x \longmapsto(y \mapsto\langle x, y\rangle)$ entstandenen Abbildung $\operatorname{id}_{S} \otimes b_{C}$.

BEweIs. Da $M$ endlich erzeugt und projektiv ist, gilt $S \otimes \operatorname{Hom}_{R}(C, R) \cong$ $\operatorname{Hom}_{S}(S \otimes C, S)$ mit $\lambda \otimes \varphi \equiv \ell_{\lambda} \cdot \varphi$ mit der Linksmultiplikation $\ell_{\lambda}$ auf $S$, siehe Korollar A.4.2. Hier gilt damit

$$
\left(\lambda \otimes b_{C}(x)\right)(\mu \otimes y)=\lambda \mu\langle x, y\rangle=\langle\lambda \otimes x, \mu \otimes y\rangle_{S}=b_{S \otimes C}(\lambda \otimes x)(\mu \otimes y) .
$$

\subsubsection{Nichtausgeartete quadratische Formen in Charakteristik 2}

Ein quadratischer Modul $(M, q)$ ist genau dann nicht nichtausgeartet, wenn ein $x \in M, x \neq 0$ mit $b(x, y)=0$ für alle $y \in M$ existiert. In diesem Fall heißen $M$ und $q$ ausgeartet. 
Es sei darauf hingewiesen, dass in der Literatur teilweise, etwa bei van der Blij und Springer [vBS59], nur solche quadratischen Formen $q$ auf $M$ als ausgeartet gezählt werden, für die ein $x \in M, x \neq 0$ mit

$$
b(x, y)=0 \forall y \in M \text { und } q(x)=0
$$

existiert. In Charakteristik 2 kämen so weitere nichtausgeartete Formen hinzu.

Van der Blij und Springer zeigen jedoch, dass für die - im nächsten Abschnitt definierten - Kompositionsalgebren über einem Körper $k$ mit $\operatorname{char}(k)=2$ lediglich der triviale Fall einer inseparablen (assoziativen und kommutativen) Körpererweiterung von $k$ zusätzlich hinzukäme.

Daher verbleiben wir bei der von Kneser oder auch von Knus gewählten, oben zuerst angegebenen Definition.

\subsection{Kompositionsalgebren über Körpern und über Ringen}

Eine quadratische Form $q$ auf einer nicht notwendig assoziativen Algebra $A$ heiße multiplikativ, wenn $q(x y)=q(x) q(y)$ für alle $x, y \in A$ gilt. Man sagt in diesem Fall auch, $(A, q)$ erlaube Komposition.

Definition. Eine Kompositionsalgebra über einem Körper $k$ ist eine nicht notwendig assoziative $k$-Algebra $C$ mit Eins zusammen mit einer multiplikativen, nichtausgearteten quadratischen Form $N$ auf $C$.

Eine Kompositionsalgebra über einem Ring $R$ ist eine nicht notwendig assoziative $R$-Algebra $C$ mit Eins, die als Modul endlich erzeugt, treu und projektiv ist, zusammen mit einer multiplikativen, nichtausgearteten quadratischen Form $N$ auf $C$.

Die quadratische Form $N$ bezeichnen wir als Norm und die zugehörige Bilinearform $\langle\cdot, \cdot\rangle$ als inneres Produkt.

Auch wenn wir es nicht explizit fordern, gilt, dass eine Kompositionsalgebra über einem Körper stets endlich-dimensional ist, siehe Satz 3.3.17.

\subsubsection{Skalarerweiterung}

Die schwache Forderung, dass die Norm nur nichtausgeartet sei, führt dazu, dass nicht jede Skalarerweiterung einer Kompositionsalgebra wieder eine solche ist. Denn die Norm ist genau dann nichtausgeartet, wenn die oben definierte Abbildung $b_{C}: C \rightarrow \operatorname{Hom}_{R}(C, R)$ injektiv ist; Injektivität bleibt jedoch beim Tensorieren nicht notwendigerweise erhalten.

Zwei mögliche Auswege wären, nur flache Skalarerweiterungen zu erlauben oder sich auf Kompositionsalgebren mit regulärer Norm $\left(b_{C}\right.$ ist bijektiv) zu 
beschränken. Der Allgemeinheit zuliebe wollen wir jedoch weitmöglichst auf die Forderung nach Regularität verzichten.

Abgesehen vom Nichtausgeartetsein der Norm verhalten sich die anderen Eigenschaften einer Kompositionsalgebra gut unter Skalarerweiterungen und bleiben erhalten.

Lemma 3.3.1. Sei $(C, N)$ eine Kompositionsalgebra über $R$ und sei $S$ eine kommutative $R$-Algebra. Ist die quadratische Form $N_{S}$ auf $S \otimes C$ nichtausgeartet, so ist $\left(S \otimes C, N_{S}\right)$ eine Kompositionsalgebra über $S$.

Beweis. Ein endlich erzeugter Modul ist genau dann treu und projektiv, wenn er treuflach und projektiv ist. Dies gilt für $C$ über $R$. Dann ist aber auch $S \otimes C$ ein endlich erzeugter treuflacher und projektiver $S$-Modul (siehe etwa [Str02, Lemma 30/1.30, Lemma 22/1.22]).

Ist die Norm $N$ auf $C$ multiplikativ, so ist es offensichtlich auch die Norm $N_{S}$ auf $S \otimes C$.

Korollar 3.3.2. Sei $(C, N)$ eine Kompositionsalgebra über $R$ und sei $S$ eine flache kommutative $R$-Algebra. Dann ist $\left(S \otimes C, N_{S}\right)$ eine Kompositionsalgebra über $S$.

BeweIs. Ist $S$ flach über $R$ und $b_{C}: C \rightarrow \operatorname{Hom}_{R}(C, R)$ injektiv, so ist auch die entsprechende Abbildung $b_{S \otimes C}$ injektiv und $N_{S}$ ist nichtausgeartet.

Reguläre Kompositionsalgebren verhalten sich gut unter jeder Skalarerweiterung.

Korollar 3.3.3. Sei $(C, N)$ eine Kompositionsalgebra über $R$, die als quadratischer Modul regulär ist, und sei $S$ eine kommutative $R$-Algebra. Dann ist $\left(S \otimes C, N_{S}\right)$ eine Kompositionsalgebra über $S$, die ebenfalls regulär ist.

Beweis. Da $(C, N)$ regulär ist, ist die Abbildung $b_{C}: C \rightarrow \operatorname{Hom}_{R}(C, R)$ bijektiv. Damit ist aber auch die Abbildung $b_{S \otimes C}$ bijektiv und somit $\left(S \otimes C, N_{S}\right)$ eine Kompositionsalgebra über $S$. Da sie als solche auch endlich erzeugt und projektiv ist, ist sie regulär.

\subsubsection{Lokalisierung}

Aus dem vorletzten Korollar folgt insbesondere, dass die Lokalisierung $C_{\mathfrak{p}}$ einer Kompositionsalgebra $C$ über $R$ für jedes Primideal $\mathfrak{p} \subset R$ eine Kompositionsalgebra über dem lokalen Ring $R_{\mathfrak{p}}$ ist.

Auch eine gewisse Umkehrung gilt - oder anders ausgedrückt: Die Eigenschaft, eine Kompositionsalgebra zu sein, ist lokal.

Lemma 3.3.4. Eine nicht notwendig assoziative $R$-Algebra $C$ mit Eins mit quadratischer Form $N$ ist genau dann eine Kompositionsalgebra, wenn sie als Modul endlich präsentiert ist und wenn für jedes maximale Ideal $\mathfrak{m} \subset R$ die 
Lokalisierung $C_{\mathfrak{m}}$ eine Kompositionsalgebra über dem lokalen Ring $R_{\mathfrak{m}}$ mit Norm $N_{\mathfrak{m}}$ ist.

Beweis. Sei also $C$ als $R$-Modul endlich präsentiert und für jedes maximale Ideal $\mathfrak{m} \subset R$ sei $C_{\mathfrak{m}}$ eine Kompositionsalgebra über $R_{\mathfrak{m}}$ mit Norm $N_{\mathfrak{m}}$.

Für jedes maximale Ideal $\mathfrak{m}$ ist dann $C_{\mathfrak{m}}$ ein projektiver und damit freier Modul über dem lokalen Ring $R_{\mathfrak{m}}$, daher ist $C$ ein projektiver $R$-Modul.

Die Algebra $C$ ist genau dann treu, wenn die Abbildung $\varphi: R \rightarrow C, r \mapsto r \cdot 1_{C}$ injektiv ist. Da für jedes maximale Ideal $\mathfrak{m}$ die Algebra $C_{\mathfrak{m}}$ treu ist, sind alle Abbildungen $\varphi_{\mathfrak{m}}: R_{\mathfrak{m}} \rightarrow C_{\mathfrak{m}}$ injektiv und damit auch $\varphi$ selbst.

$\mathrm{Zu} x, y \in C$ sei $\lambda=N(x y)-N(x) N(y)$. Für jedes maximale Ideal $\mathfrak{m} \subset R$ gilt dann $\frac{\lambda}{1}=N_{\mathfrak{m}}\left(\frac{x y}{1}\right)-N_{\mathfrak{m}}\left(\frac{x}{1}\right) N_{\mathfrak{m}}\left(\frac{y}{1}\right)=0$ in $R_{\mathfrak{m}}$, denn $N_{\mathfrak{m}}$ ist multiplikativ. Daher muss auch $\lambda=0$ in $C$ gelten. Folglich ist auch $N$ multiplikativ.

Da die Lokalisierung $b_{C_{\mathfrak{m}}}: C_{\mathfrak{m}} \rightarrow \operatorname{Hom}_{R_{\mathfrak{m}}}\left(C_{\mathfrak{m}}, R_{\mathfrak{m}}\right), x \longmapsto(y \mapsto\langle x, y\rangle)$ in jedem maximalen Ideal $\mathfrak{m}$ injektiv ist, ist auch die Abbildung $b_{C}: C \rightarrow \operatorname{Hom}_{R}(C, R)$ selbst injektiv. Somit ist $N$ nichtausgeartet.

\subsubsection{Gute Reduktion}

Da der Quotientenkörper $R / \mathfrak{m}$ zu einem maximalen Ideal $\mathfrak{m} \subset R$ als $R$-Modul in der Regel nicht flach ist, wird die Quotientenalgebra einer Kompositionsalgebra modulo $\mathfrak{m}$ im Allgemeinen keine Kompositionsalgebra sein.

Definition. Eine Kompositionsalgebra $C$ über $R$ habe gute Reduktion in einem Primideal $\mathfrak{p} \subset R$, wenn die Quotientenalgebra $C / \mathfrak{p} C$ eine Kompositionsalgebra über dem Restklassenring $R / \mathfrak{p}$ ist. Eine Kompositionsalgebra $C$ über $R$ habe gute Reduktion (insgesamt), wenn $C$ in jedem maximalen Ideal $\mathfrak{m} \subset R$ gute Reduktion hat.

Lemma 3.3.5. Eine Kompositionsalgebra $C$ über $R$ hat genau dann gute Reduktion, wenn sie als quadratischer Modul regulär ist.

BEweis. Da $C$ endlich erzeugt und projektiv ist, ist $C$ genau dann regulär, wenn die Abbildung $b_{C}: C \rightarrow \operatorname{Hom}_{R}(C, R), x \longmapsto(y \mapsto\langle x, y\rangle)$ bijektiv ist. Diese ist genau dann bijektiv, wenn für jedes maximale Ideal $\mathfrak{m} \subset R$ die Quotientenabbildung $b_{C / \mathfrak{m} C}: C / \mathfrak{m} C \rightarrow \operatorname{Hom}_{R / \mathfrak{m}}(C / \mathfrak{m} C, R / \mathfrak{m})$ bijektiv ist (siehe Korollar A.3.4 im Anhang). Da $C / \mathfrak{m} C$ und $\operatorname{Hom}_{R / \mathfrak{m}}(C / \mathfrak{m} C, R / \mathfrak{m})$ dieselbe endliche Dimension über dem Körper $R / \mathfrak{m}$ haben, ist diese Abbildung genau dann bijektiv, wenn sie injektiv ist. Dies ist für jedes $\mathfrak{m}$ genau dann der Fall, wenn $C$ gute Reduktion hat.

Allgemeiner kann man auch beliebige nicht notwendig assoziative Algebren mit quadratischer Form betrachten, deren sämtliche Quotientenalgebren Kompositionsalgebren sind. 
Lemma 3.3.6. Sei $C$ eine nicht notwendig assoziative $R$-Algebra mit Eins mit quadratischer Form $N$, die als $R$-Modul endlich erzeugt und projektiv ist und für die für jedes maximale Ideal $\mathfrak{m} \subset R$ die Quotientenalgebra $C / \mathfrak{m} C$ eine Kompositionsalgebra über dem Restklassenkörper $R / \mathfrak{m}$ mit Norm $N_{R / \mathfrak{m}}$ ist. Dann ist $(C, N)$ eine reguläre Kompositionsalgebra.

Beweis. Aus dem Beweis des letzten Lemmas folgt, dass $(C, N)$ genau dann ein regulärer quadratischer $R$-Modul ist, wenn $\left(C / \mathfrak{m} C, N_{R / \mathfrak{m}}\right)$ ein nichtausgearteter $R / \mathfrak{m}$-Modul für jedes maximale Ideal $\mathfrak{m} \subset R$ ist.

Zu $x, y \in C$ sei $\lambda=N(x y)-N(x) N(y)$. Nach Korollar A.3.2 gilt $\lambda=0$ genau dann, wenn $\lambda+\mathfrak{m}=N_{R / \mathfrak{m}}(x y+\mathfrak{m})-N_{R / \mathfrak{m}}(x+\mathfrak{m}) N_{R / \mathfrak{m}}(y+\mathfrak{m})=0$ in $R / \mathfrak{m}$ für jedes maximale Ideal $\mathfrak{m}$ gilt. Daher ist die Norm $N$ genau dann multiplikativ, wenn alle Normen $N_{R / \mathfrak{m}}$ multiplikativ sind.

Ist für jedes $\mathfrak{m}$ die Quotientenalgebra $C / \mathfrak{m} C$ eine Kompositionsalgebra über dem Körper $R / \mathfrak{m}$, so sind insbesondere alle Reduktionen $R / \mathfrak{m} \rightarrow C / \mathfrak{m} C$ der Abbildung $\varphi: R \rightarrow C, r \mapsto r \cdot 1_{C}$ injektiv. Damit ist auch $\varphi$ selbst injektiv und somit $C$ treu über $R$.

Sei $\mathfrak{m} \subset R$ ein maximales Ideal. Da Lokalisierung und Quotientenbildung vertauschen, genauer da $R / \mathfrak{m} \cong R_{\mathfrak{m}} / \mathfrak{m} R_{\mathfrak{m}}$ und $C / \mathfrak{m} C \cong C_{\mathfrak{m}} /\left(\mathfrak{m} R_{\mathfrak{m}}\right) C_{\mathfrak{m}}$ gelten, bleibt gute Reduktion bei der Lokalisierung in $\mathfrak{m}$ erhalten.

\subsubsection{Norm der Eins, quadratische Gleichung, Konjugation}

Lemma 3.3.7. Sei $(C, N)$ eine Kompositionsalgebra über $R$, und sei e $\in C$ ihr Einselement. Dann gilt $N(e)=1$.

Beweis. Für jedes $x \in C$ gilt $N(x)=N(x e)=N(x) N(e)$. Ist $R$ nullteilerfrei, so folgt die Behauptung direkt. Allgemein gilt $\langle x, e\rangle=N(x+e)-N(x)-N(e)=$ $(N(x+e)-N(x)-N(e)) N(e)=\langle x, N(e) e\rangle$, also $\langle x,(1-N(e)) e\rangle=0$ für jedes $x \in C$. Da $N$ nichtausgeartet ist, folgt $(1-N(e)) e=0$. Damit liegt $1-N(e)$ im Annullator von $C$, der jedoch trivial ist, da $C$ treu über $R$ ist.

Korollar 3.3.8. Es ist $R$ genau dann eine Kompositionsalgebra über sich selbst, wenn 2 kein Nullteiler in $R$ ist. Insbesondere ist $R$ genau dann regulär, wenn 2 eine Einheit in $R$ ist.

Ist $R$ eine Kompositionsalgebra, so erfüllt die Norm $N(\lambda)=\lambda^{2}$ für alle $\lambda \in R$.

Lemma 3.3.9 ([Knu91, aus V(7.1)]). Sei $(C, N)$ eine Kompositionsalgebra über $R$ mit Einselement e. Dann erfüllt jedes Element $x \in C$ eine quadratische Gleichung, es gilt

$$
x^{2}-\langle x, e\rangle x+N(x) e=0 .
$$

Ist $R=k$ ein Körper, so ist folglich jedes Element $x \in C$ algebraisch über $k$ und für $x \notin k e$ ist die obige Gleichung die Minimalgleichung von $x$. 
Das Element $\operatorname{Tr}(x):=\langle x, e\rangle \in R$ nennt man die Spur von $x$; die Gleichung hat dann die Form $x^{2}-\operatorname{Tr}(x) x+N(x) e=0$.

Lemma 3.3.10 ([Knu91, aus V (7.1)]). Sei $(C, N)$ eine Kompositionsalgebra über $R$ mit Einselement e. Dann erfüllt die Konjugation

$$
\bar{x}:=\langle x, e\rangle e-x
$$

die folgenden Eigenschaften

$$
\begin{gathered}
\overline{x+y}=\bar{x}+\bar{y}, \quad \overline{x y}=\bar{y} \bar{x}, \quad \overline{\lambda e}=\lambda e, \quad \overline{\bar{x}}=x, \\
x \bar{x}=\bar{x} x=N(x) e, \quad N(\bar{x})=N(x), \quad\langle\bar{x}, \bar{y}\rangle=\langle x, y\rangle
\end{gathered}
$$

für alle $x, y \in C$ und $\lambda \in R$.

Die Konjugation lässt sich auch durch die Spiegelung $s_{e}$ an der zu $e$ orthogonalen Hyperebene beschreiben; es gilt $\bar{x}=-s_{e}(x)$.

Neben den Formeln in (3.1) gelten auch $x+\bar{x}=\operatorname{Tr}(x) e \in R e$ und $x \bar{x}=N(x) e \in$ $R e$, daher ist die Konjugation insbesondere eine Standard-Involution auf $C$. Da $C$ als $R$-Modul endlich erzeugt, projektiv und treu ist, gibt es nur diese eine Standard-Involution auf $C$ (siehe etwa [Knu91, I (1.3.4) Proposition]).

Ist umgekehrt auf einer treuen, nicht notwendig assoziativen $R$-Algebra $A$ eine Standard-Involution $A \rightarrow A, x \mapsto \bar{x}$ gegeben, d.h. die Abbildung erfüllt die Regeln (3.1) sowie $x \bar{x} \in R e$ und $x+\bar{x} \in R e$, so lässt sich über die Vorschrift $N(x):=x \bar{x}$ eine multiplikative quadratische Form auf $A$ definieren.

Lemma 3.3.11 ([Knu91, aus V (7.1)]). Sei $(C, N)$ eine Kompositionsalgebra über $R$. Dann gelten die folgenden Identitäten

$$
\begin{aligned}
& \langle x y, z\rangle=\langle y, \bar{x} z\rangle=\langle x, z \bar{y}\rangle, \\
& x(\bar{x} y)=N(x) y, \quad(y x) \bar{x}=N(x) y, \\
& x(x y)=(x x) y, \quad(y x) x=y(x x), \quad x(y x)=(x y) x
\end{aligned}
$$

für alle $x, y, z \in C$.

Die ersten Umformungen (3.2) der Bilinearform sind für das im nächsten Abschnitt folgende Verdopplungsverfahren hilfreich. Die beiden Formeln (3.3) bestimmen unter anderem das Aussehen von Inversen, siehe Lemma 3.3.20.

Korollar 3.3.12. Sei $(C, N)$ eine Kompositionsalgebra über $R$. Ist $x \in C$ ein Element, dessen Norm $N(x)$ kein Nullteiler in $R$ ist, so ist $x$ linear unabhängig.

Beweis. Sei $\lambda x=0$. Dann gilt unter Verwendung der Formeln (3.2) und (3.3) für alle $y \in C$ die Gleichheit $0=\langle\lambda x, x y\rangle=\langle\lambda e, \bar{x}(x y)\rangle=N(x)\langle\lambda e, y\rangle$. Ist $N(x)$ kein Nullteiler, so folgt, da $N$ nichtausgeartet ist, $\lambda e=0$. Da $C$ treu ist, muss $\lambda=0$ sein. 
Aus den letzten drei Identitäten (3.4) des Lemmas folgt, dass der trilineare Assoziator $\{x, y, z\}=(x y) z-x(y z)$ eine alternierende Funktion ist. Daher ist jede Kompositionsalgebra eine alternative Algebra, insbesondere gelten die folgenden Aussagen:

Satz 3.3.13. Sei $(C, N)$ eine Kompositionsalgebra über $R$. Für jedes $x \in C$ ist die von $x$ erzeugte Unteralgebra $R[x]$ assoziativ und kommutativ. Jede von zwei Elementen $x, y \in C$ erzeugte Unteralgebra $R[x, y]$ ist assoziativ.

Die erste Aussage folgt alternativ bereits aus der quadratischen Gleichung in Lemma 3.3.9. Außerdem besagt jenes Lemma, dass für die von $x$ erzeugte Unteralgebra $R[x]=R+R x$ gilt. Die zweite Aussage folgt aus E. Artins Theorem, dass allgemeiner in einer alternativen Algebra jede von nur zwei Elementen erzeugte Unteralgebra assoziativ ist. Der von Schafer [Sch66, Theorem 3.1] angegebene Beweis für alternative Algebren über Körpern behält seine Gültigkeit auch für die entsprechenden Algebren über beliebigen Ringen.

Die Einschränkung der Norm auf die Unteralgebren $R[x]$ bzw. $R[x, y]$ kann ausgeartet sein; in diesem Fall sind diese keine Kompositionsalgebren.

\subsubsection{Die Struktur von Kompositionsalgebren}

Eine Unterkompositionsalgebra einer Kompositionsalgebra ist ein endlich erzeugter, projektiver Untermodul, der multiplikativ abgeschlossen ist, die Eins enthält und auf dem die Einschränkung der Norm nichtausgeartet ist.

Im folgenden Satz werden wir auch beliebige Unteralgebren, also multiplikativ abgeschlossene Untermoduln, die die Eins enthalten, betrachten.

Satz 3.3.14. Sei $B$ eine echte Unteralgebra einer Kompositionsalgebra $(C, N)$ über $R$ und sei $v \in B^{\perp}$ so, dass $\lambda=-N(v)$ kein Nullteiler in $R$ ist. Dann ist $B \oplus B v$ eine Unteralgebra von $C$ und es gelten für die Multiplikation, die Norm, die Bilinearform und das Konjugierte in $B \oplus B v$ die Formeln

$$
\begin{aligned}
(a+b v)(c+d v) & =(a c+\lambda \bar{d} b)+(d a+b \bar{c}) v, \\
N(a+b v) & =N(a)-\lambda N(b), \\
\langle a+b v, c+d v\rangle & =\langle a, c\rangle-\lambda\langle b, d\rangle, \\
\overline{a+b v} & =\bar{a}-b v
\end{aligned}
$$

für $a, b, c, d \in B$. Außerdem ist $B$ assoziativ, und $B \oplus B v$ ist genau dann assoziativ, wenn $B$ kommutativ ist.

Ist $B$ mit der eingeschränkten Norm nichtausgeartet, so ist auch $B \oplus B v$ nichtausgeartet. Insbesondere ist auch $B \oplus B v$ eine Unterkompositionsalgebra, wenn $B$ eine ist. Ist $B$ regulär und zusätzlich $\lambda=-N(v) \in R^{\times}$eine Einheit, so ist auch $B \oplus B v$ regulär. 
Die Hauptaussagen beweist Knus in [Knu91, V (7.1.7) Lemma]; die weitergehenden Forderungen, dass durchgehend $\lambda=-N(v)$ eine Einheit und $B$ regulär (nonsingular im Original) sein muss, werden dabei großteils nicht verwendet. Die angegebenen Formeln gelten übrigens auch dann auf $B \oplus B v$, wenn $\lambda=-N(v)$ ein Nullteiler sein sollte.

Beweis (fehlende Aussagen). Die Formel für die Bilinearform folgt ebenso wie die Formel für das Konjugierte direkt aus der Formel für die Norm.

Ist $B$ kommutativ und assoziativ, so rechnet man direkt nach, dass die Multiplikation auf $B \oplus B v$ assoziativ ist.

Sei $a+b v \in B \oplus B v$ so, dass $0=\langle a+b v, c+d v\rangle=\langle a, c\rangle-\lambda\langle b, d\rangle$ für alle $y=c+d v \in B \oplus B v$ gilt. Setzen wir $d=0$ bzw. $c=0$, so folgt, dass insbesondere $\langle a, c\rangle=0$ für alle $c \in B$ und dass $-\lambda\langle b, d\rangle=0$ für alle $d \in B$ gilt. Ist $N$ auf $B$ nichtausgeartet, so folgt, da $\lambda$ kein Nullteiler ist, dass $a=b=0$ ist. Folglich ist auch $N$ auf $B \oplus B v$ nichtausgeartet.

Ist $B$ eine Unterkompositionsalgebra, so ist mit $B$ auch $B v$ und damit auch $B \oplus B v$ endlich erzeugt und projektiv, denn $v$ ist nach Korollar 3.3.12 linear unabhängig. Außerdem ist auch jede Unteralgebra von $C$ treu.

Ist $\lambda=-N(v)$ eine Einheit, so überträgt sich die Regularität von $B$ auf $B v$. Da $v \in B^{\perp}$ gilt, ist dann auch $B \oplus B v$ regulär.

Ist $C$ eine Kompositionsalgebra über einem Körper, so existiert zu jedem nichtausgearteten Untervektorraum $B \subsetneq C$ stets ein Element $v \in B^{\perp}$ mit nichttrivialer Norm $N(v) \neq 0$.

Wenn $C$ entsprechend eine reguläre Kompositionsalgebra über einem lokalen Ring $R$ ist, existiert zu jedem regulären Untermodul $B \subsetneq C$ stets ein Element $v \in B^{\perp}$ mit invertierbarer Norm $N(v) \in R^{\times}$(siehe [Knu91, Beweis zu $\mathrm{V}(7.1 .6)$ Theorem]).

Umgekehrt lässt sich mit dem Verdopplungsverfahren aus einer Kompositionsalgebra $B$ eine neue, größere Algebra konstruieren.

Korollar 3.3.15. Sei $(B, N)$ eine Kompositionsalgebra über $R$ und $\lambda \in R^{\times}$ eine Einheit. Sei $v$ eine Unbestimmte, dann definieren die Formeln (3.5) und (3.6) eine Multiplikation und eine multiplikative quadratische Form auf $C=B \oplus B v$.

Ist $B$ assoziativ, so ist $C=B \oplus B v$ eine Kompositionsalgebra über $R$. Es ist $C$ genau dann assoziativ, wenn $B$ assoziativ und kommutativ ist.

Man sagt, die Algebra $B \oplus B v$ sei aus $B$ durch Verdopplung entstanden.

Ist $B$ nicht assoziativ, so lassen sich zwar wie angegeben eine Multiplikation und eine quadratische Form auf $B \oplus B v$ definieren, diese sind jedoch nicht miteinander verträglich. Die neue Algebra $B \oplus B v$ ist in diesem Fall daher keine Kompositionsalgebra. 
Die Verdopplungsmöglichkeiten innerhalb einer Kompositionsalgebra erlauben, ihre Struktur, insbesondere ihren Rang genauer zu bestimmen.

Lemma 3.3.16. Sei $\operatorname{char}(R)=2$, und sei $(C, N)$ eine Kompositionsalgebra über R. Sei $a \in C$ ein Element, dessen Spur $\langle a, e\rangle$ kein Nullteiler in $R$ ist. Dann ist Re $\oplus$ Ra eine Unterkompositionsalgebra in $C$ von Rang 2.

Beweis. Ist $\lambda e+\mu a=0$, so gelten $0=\langle\lambda e+\mu a, a\rangle=\lambda\langle e, a\rangle+2 \mu N(a)=$ $\lambda\langle a, e\rangle$ und $0=\langle\lambda e+\mu a, e\rangle=\mu\langle a, e\rangle$. Da $\langle a, e\rangle$ kein Nullteiler ist, folgt $\lambda=\mu=0$. Somit ist $R e \oplus R a$ ein freier Modul von Rang 2 mit Basis $e, a$. Da $d(e, a)=\langle a, e\rangle^{2}$ ebenfalls kein Nullteiler ist, ist $N$ auf $R e \oplus R a$ nichtausgeartet. Da jedes $x$ in $C$ eine quadratische Gleichung $x^{2}-\langle a, e\rangle x+N(x)$ erfüllt (siehe Lemma 3.3.9), ist $R e \oplus R a$ multiplikativ abgeschlossen und somit eine Unterkompositionsalgebra.

Ist $R$ ein Integritätsbereich (beliebiger Charakteristik), so existiert in einer Kompositionsalgebra $C$ stets ein Element $a$ mit nichttrivialer Spur $\langle a, e\rangle \neq 0$, andernfalls wäre $C$ ausgeartet. Ist $C$ eine reguläre Kompositionsalgebra über einem beliebigen Ring, so existiert ebenso stets ein $a \in C$ mit $\langle a, e\rangle=1$.

Da über einem Körper $k$ in jeder Kompositionsalgebra als Unterkompositionsalgebra $k e$ (falls char $(k) \neq 2$ ) oder eine quadratische Algebra $k e \oplus k a$ vorkommt, kann jede Kompositionsalgebra über einem Körper durch wiederholte Verdopplung konstruiert werden. Über Ringen ist das im Allgemeinen nicht möglich, wie wir in Bemerkung 3.4.3 zeigen werden.

Die Bedingungen an Assoziativität und Kommutativität innerhalb des Verdopplungsverfahrens führen dazu, dass Kompositionsalgebren über Körpern nur wenige und kleine Dimensionen annehmen können. Insbesondere muss über Körpern nicht gefordert werden, dass die Algebra endlich-dimensional ist (vergleiche hierzu auch die Definition und die anschließenden Bemerkungen bei Springer und Veldkamp [SV00, Definition 1.2.1]):

Hauptsatz 3.3.17 ([SV00, aus Theorem 1.6.2]). Die möglichen Dimensionen von Kompositionsalgebren über Körpern sind 1 (nur für char $\neq 2$ ), 2, 4 und 8.

Die lokale Natur der Eigenschaften einer Kompositionsalgebra führt dazu, dass Kompositionsalgebren auch über Ringen lokal nur gewisse Ränge annehmen können. Dabei muss der Rang einer Kompositionsalgebra über einem beliebigen Ring im Allgemeinem nur lokal, nicht global konstant sein (siehe Abschnitt 3.4.1). Ist $R$ jedoch ein Ring, dessen Spektrum zusammenhängend ist, so ist der Rang eines jeden projektiven $R$-Moduls konstant. Dies ist insbesondere für Integritätsbereiche der Fall.

Hauptsatz 3.3.18 ([Knu91, aus V (7.1.6) Theorem]). Sei $C$ eine Kompositionsalgebra über $R$ von konstantem Rang. Dann ist der Rang von $C$ entweder 1, 2, 4 oder 8 . 
Rang 1 tritt genau dann auf, wenn 2 kein Nullteiler ist; in diesem Fall gilt $C \cong R$. Kompositionsalgebren von Rang 2 sind assoziativ und kommutativ, also quadratische Algebren. Kompositionsalgebren von Rang 4 sind verallgemeinerte Quaternionenalgebren, also insbesondere assoziativ, aber nicht kommutativ. Kompositionsalgebren von Rang 8 sind weder assoziativ noch kommutativ.

Dabei heißt eine (assoziative) Algebra quadratisch, wenn sie als Modul projektiv und von Rang 2 ist. Quadratische Algebren sind stets kommutativ und besitzen eine (eindeutige) Standard-Involution. Eine quadratische Algebra ist bezüglich der zur Standard-Involution gehörigen Norm genau dann regulär, wenn sie separabel ist (siehe [Knu91, I (7.3.4)]).

Eine (assoziative) Algebra heißt verallgemeinerte Quaternionenalgebra, wenn sie als Modul projektiv und von Rang 4 ist und wenn sie eine Standard-Involution besitzt. Ist eine verallgemeinerte Quaternionenalgebra regulär bezüglich der zur Standard-Involution gehörigen Norm, so ist sie eine Azumaya-Algebra. Umgekehrt besitzt jede Azumaya-Algebra von Rang 4 eine Standard-Involution, und bezüglich der zur Standard-Involution gehörigen Norm ist sie regulär (siehe [Knu91, I (7.3.5)]); insbesondere ist sie eine Kompositionsalgebra.

Interessant sind an dieser Stelle insbesondere die bisher noch nicht behandelten, stets nichtassoziativen Kompositionsalgebren von Rang 8.

Definition. Eine Kompositionsalgebra von Dimension 8 über einem Körper heißt Oktaven- oder Oktonionenalgebra oder auch Cayley-Algebra.

Eine Kompositionsalgebra von Rang 8 über einem Ring heiße Oktavenalgebra.

Mittels des Verdopplungsverfahrens lassen sich aus Quaternionenalgebren Oktavenalgebren konstruieren. Über Körpern gilt nicht nur die Umkehrung, dass jede Oktavenalgebra aus Verdopplung einer Quaternionenunteralgebra entsteht, sondern darüber hinaus auch:

Lemma 3.3.19 ([SV00, Proposition 1.6.4]). Jedes Element einer Oktavenalgebra über einem Körper ist in einer Quaternionenunteralgebra enthalten.

\subsubsection{Divisions- und zerfallende Kompositionsalgebren}

Sei $A$ eine nicht notwendig assoziative Algebra. Es heißt $y \in A$ Inverses zu $x \in A$, falls

$$
x(y z)=y(x z)=(z x) y=(z y) x=z
$$

für alle $z \in A$ gilt. Existiert ein Inverses zu $x$, ist es eindeutig. Es heißt $x \in A$ Nullteiler, falls es ein Element $y \in A, y \neq 0$ mit $x y=0$ oder $y x=0$ gibt.

Lemma 3.3.20. Sei $(C, N)$ eine Kompositionsalgebra über $R$, und sei $x \in C$. Dann ist $x$ genau dann invertierbar, wenn $N(x)$ eine Einheit in $R$ ist; das Inverse ist dann $x^{-1}=N(x)^{-1} \bar{x}$. Außerdem ist $x$ genau dann ein Nullteiler in $C$, wenn $N(x)$ ein Nullteiler in $R$ ist. 
Beweis. Da $N(x)=N(\bar{x})$ und $x(\bar{x} y)=N(x) y=(y x) \bar{x}$ für alle $y \in C$ gilt (siehe Lemma 3.3.10 und 3.3.11), folgt die Aussage für das Inverse.

Ist $\lambda=N(x)$ kein Nullteiler in $R$, so enthält die von $\lambda$ erzeugte multiplikative Menge $S=\left\{\lambda^{n} \mid n \in \mathbb{N}_{0}\right\}$ ebenfalls keinen Nullteiler. Daher ist $S^{-1} R$ flach über $R$, es ist $C^{\prime}=S^{-1} R \otimes_{R} C$ eine Kompositionsalgebra über $S^{-1} R$ und die kanonische Abbildung $C \rightarrow C^{\prime}, y \mapsto 1 \otimes y$ ist injektiv. In $C^{\prime}$ ist $1 \otimes x$ ein Element, dessen Norm $N(1 \otimes x)=\lambda$ eine Einheit ist, und somit selbst eine Einheit. Daher kann $1 \otimes x$ in $C^{\prime}$ und damit auch $x$ in $C$ kein Nullteiler sein. Es folgt, dass die Norm eines Nullteiler ein Nullteiler ist.

Da $x \bar{x}=N(x) e$ gilt, ist $x$ ein Nullteiler, wenn $N(x)=0$ gilt. Sei nun $N(x) \neq 0$ ein Nullteiler in $R$, etwa $\lambda N(x)=0$ mit $\lambda \neq 0$. Da $C$ treu ist, ist auch $\lambda e \neq 0$ in $C$. Entweder ist $\lambda e$ ein Nullteilerpartner zu $x$, nämlich genau dann, wenn $\lambda \bar{x}=0$ und damit auch $\lambda x=0$ gilt, oder $\lambda \bar{x}$ ist ein passender Partner, denn in jedem Fall gilt $x(\lambda \bar{x})=\lambda N(x) e=0$.

Korollar 3.3.21. Jedes Element $x \neq 0$ einer Kompositionsalgebra über einem Körper ist entweder eine Einheit oder ein Nullteiler.

Die quadratische Form $q$ eines Modul $(M, q)$ heiße anisotrop, wenn jedes Element anisotrop ist, d. h. wenn $q(x) \neq 0$ für alle $x \in M, x \neq 0$ ist.

Andernfalls existiert ein isotropes Element $x \neq 0$ mit $q(x)=0$ und die quadratische Form heiße ebenfalls isotrop.

Sei $(C, N)$ eine Kompositionsalgebra über einem Körper $k$. Ist die quadratische Form $N$ anisotrop, so ist nach dem obigen Lemma jedes Element ungleich 0 in $C$ invertierbar; die Algebra ist eine Kompositionsdivisionsalgebra.

Andernfalls existiert ein isotropes Element $x \neq 0$. In diesem Fall lässt sich eine zweidimensionale Unterkompositionsalgebra $D=k e \oplus k a$ von $C$ konstruieren, die ein ebenfalls isotropes Element $x^{\prime} \neq 0$ enthält; siehe Springer und Veldkamp [SV00, Abschnitt 1.8]. Da sich $C$ durch null- bis zweifache Verdopplung aus $D$ ergibt und da bei diesem Prozess auch die isotropen Elemente "verdoppelt" werden, enthält $C$ einen total isotropen Unterraum von Dimension $\frac{1}{2} \operatorname{dim} C$. Als Kompositionsalgebra ist $C$ selbst nichtausgeartet, kann also keinen größeren isotropen Unterraum enthalten, sodass ihr Witt-Index - das ist die Dimension eines jeden maximalen total isotropen Unterraumes - ebenfalls $\frac{1}{2} \operatorname{dim} C$ beträgt.

Definition. Existiert in einer Kompositionsalgebra über einem Körper ein isotropes Element $x \neq 0$, so heiße die Kompositionsalgebra zerfallend.

Aus den obigen Anmerkungen folgt über Körpern die folgende Aussage:

Lemma 3.3.22. Eine Kompositionsalgebra $C$ über einem Körper ist entweder eine Divisionsalgebra oder ihr Witt-Index beträgt $\frac{1}{2} \operatorname{dim} C$ und sie zerfällt.

Der Struktursatz von Wedderburn bestätigt im Falle von Quaternionenalgebren $A$ über Körpern $k$ diese Aussage. Entweder ist $A$ eine Divisionsalgebra oder isomorph zu einem vollen Matrixring $\mathbb{M}_{2}(k)$. 
Vorgreifend auf Ergebnisse des nächsten Abschnittes sei hier bereits die Eindeutigkeit zerfallender Kompositionsalgebren erwähnt:

Hauptsatz 3.3.23 ([SV00, Theorem 1.8.1]). Sei $k$ ein Körper. In jeder der Dimensionen 2, 4 und 8 existiert bis auf Isomorphie genau eine zerfallende Kompositionsalgebra über $k$.

\subsubsection{Zusammenhang von Norm und Multiplikation}

Da nach Lemma 3.3.9 jedes Element $x$ einer Kompositionsalgebra $(C, N)$ eine quadratische Gleichung $x^{2}-\langle x, e\rangle x+N(x) e=0$ erfüllt, besteht ein gewisser Zusammenhang zwischen der Multiplikation und der Norm.

Korollar 3.3.24. Die Norm einer Kompositionsalgebra über einem Integritätsbereich ist eindeutig durch ihre Algebrenstruktur bestimmt.

BewEIS (nach [SV00, Corollary 1.2.4]). Sei $(C, N)$ eine Kompositionsalgebra mit Eins $e$ über dem Integritätsbereich $R$ und sei $k$ dessen Quotientenkörper. Dann ist $k$ flach über $R$ und mithin $C_{k}=k \otimes C$ eine Kompositionsalgebra über $k$ mit Norm $N_{k}$. Für $x=\lambda e$ gilt $N_{k}(\lambda e)=\lambda^{2}$, für $x \in C_{k} \backslash k e$ ist die obige quadratische Gleichung die Minimalgleichung von $x$ und damit ihr konstanter Koeffizient $N_{k}(x)$ eindeutig bestimmt. Für $x \in C$ ist $N(x)=N_{k}(1 \otimes x)$.

Im weiteren Abschnitt wollen wir zeigen, dass über Körpern auch eine gewisse Umkehrung gilt. Dazu definieren und betrachten wir zunächst die strukturerhaltenden Abbildungen von quadratischen Moduln und von Kompositionsalgebren.

Definition. Eine Ähnlichkeit zwischen zwei quadratischen Moduln $\left(M_{1}, q_{1}\right)$ und $\left(M_{2}, q_{2}\right)$ über $R$ ist ein bijektiver $R$-Modulhomomorphismus $t: M_{1} \rightarrow M_{2}$, für den es eine Einheit $n(t) \in R^{\times}$so gibt, dass

$$
q_{2}(t(x))=n(t) q_{1}(x)
$$

für alle $x \in M_{1}$ gilt. Die Einheit $n(t)$ heißt Vielfaches von $t$. Eine Isometrie ist eine Ähnlichkeit mit Vielfachem $n(t)=1$.

Quadratische Räume, zwischen denen eine Ähnlichkeit oder Isometrie existiert, heißen dementsprechend ähnlich bzw. isometrisch.

Ist $t$ eine Ähnlichkeit mit Vielfachem $n(t)$, so gilt für die zugehörigen Bilinearformen $\langle t(x), t(y)\rangle_{2}=n(t)\langle x, y\rangle_{1}$ für $x, y \in M_{1}$.

Ist $\left(M_{1}, q_{1}\right)$ nichtausgeartet und ist $t: M_{1} \rightarrow M_{2}$ ein $R$-Modulhomomorphismus, der die Bedingung (3.7) erfüllt, so ist er automatisch injektiv - dies gilt bereits, wenn $n(t)$ kein Nullteiler in $R$ ist. Ist zusätzlich $t$ surjektiv, so ist auch $\left(M_{2}, q_{2}\right)$ nichtausgeartet. 
Definition. Eine Isomorphie zwischen zwei Kompositionsalgebren $\left(C_{1}, N_{1}\right)$ und $\left(C_{2}, N_{2}\right)$ über $R$ ist ein bijektiver $R$-Modulhomomorphismus $t: C_{1} \rightarrow C_{2}$, der $t(x y)=t(x) t(y)$ und $t(\bar{x})=\overline{t(x)}$ für alle $x, y \in C_{1}$ erfüllt.

Da die Norm und die Konjugation über die Formel $N(x) e=x \bar{x}$ zusammenhängen, ist ein bijektiver multiplikativer $R$-Modulhomomorphismus $t: C_{1} \rightarrow C_{2}$ genau dann mit der Konjugation verträglich, wenn er mit der Norm verträglich ist $\left(N_{2}(t(x))=N_{1}(x)\right.$ für alle $\left.x \in C_{1}\right)$, also genau dann, wenn er eine Isometrie ist.

Über gewissen Ringen ist die Forderung nach der Verträglichkeit eines Isomorphismus mit der Konjugation bzw. der Äquivalenz der Normen automatisch erfüllt (für Körper siehe etwa [SV00, Definition 1.2.1, Corollary 1.2.4]):

Lemma 3.3.25. Seien $\left(C_{1}, N_{1}\right)$ und $\left(C_{2}, N_{2}\right)$ zwei Kompositionsalgebren über einem Integritätsbereich $R$. Dann ist jeder bijektive multiplikative $R$-Modulhomomorphismus $t: C_{1} \rightarrow C_{2}$ eine Isometrie, mithin eine Isomorphie von Kompositionsalgebren.

Beweis. Wie im Beweis zu Korollar 3.3.24 reicht es, die Aussage für den Quotientenkörper $k$ von $R$ zu beweisen.

Für $x=\lambda e_{1} \in k e_{1}$ gilt $N_{2}\left(t\left(\lambda e_{1}\right)\right)=N_{2}\left(\lambda e_{2}\right)=\lambda^{2}=N_{1}\left(\lambda e_{1}\right)$. Für $x \notin k e_{1}$ ist $t(x) \notin k e_{2}$ und $t(x)^{2}-\left\langle x, e_{1}\right\rangle t(x)+N_{1}(x) e_{2}=t\left(x^{2}-\left\langle x, e_{1}\right\rangle x+N_{1}(x) e_{1}\right)=0$ ist die eindeutige Minimalgleichung von $t(x)$, folglich gilt für den konstanten Koeffizienten $N_{1}(x)=N_{2}(t(x))$.

Wir kommen nun über Körpern zu der Umkehrung, dass die metrische Struktur, hier also die Norm, bereits die Kompositionsalgebra bis auf Isomorphie bestimmt.

Hauptsatz 3.3.26 ([SV00, Theorem 1.7.1]). Ähnliche, insbesondere isometrische Kompositionsalgebren über einem Körper sind isomorph.

Ein vollständiger Beweis findet sich bei Springer und Veldkamp, wir benötigen nur den Fall für $k^{\prime}=k$ und die Identität $\sigma$. Es sei hier eine kurze Zusammenfassung angegeben:

BeweIs (Skizze). Zunächst wird die Ähnlichkeit $t^{\prime}: C_{1} \rightarrow C_{2}$ durch die Isometrie $t: C_{1} \rightarrow C_{2}, x \mapsto t^{\prime}(e)^{-1} t^{\prime}(x)$, die die Eins auf die Eins abbildet, ersetzt. Es seien $D_{0}=k e$ und $\varphi_{0}=t$. Mit Hilfe des Verdopplungsverfahrens werden auf geeigneten Unterkompositionsalgebren Isomorphismen

$$
\begin{aligned}
\varphi_{i+1}: D_{i+1}=D_{i} \oplus D_{i} a_{i+1} & \rightarrow D_{i+1}^{\prime}=D_{i}^{\prime} \oplus D_{i}^{\prime} a_{i+1}^{\prime} \\
x+y a_{i+1} & \mapsto \varphi_{i}(x)+\varphi_{i}(y) a_{i+1}^{\prime}
\end{aligned}
$$

für $0 \leq i \leq 2$ (je nach Dimension von $C_{1}$ ) definiert. Die $a_{i+1}$ sind dabei jeweils Elemente aus $D_{i}^{\perp}$ mit $N\left(a_{i+1}\right) \neq 0$ (bzw. $\left\langle a_{1}, e_{1}\right\rangle=1$ in char $\left.=2\right)$ und es ist 
$a_{1}^{\prime}=t\left(a_{1}\right)$ und $a_{2}^{\prime}=t\left(a_{2}\right)$. Ist $\operatorname{dim} C_{1}=8$, so wird $\varphi_{2}$ nach Witts Theorem zu einer Isometrie $u: C_{1} \rightarrow C_{2}$ fortgesetzt und $a_{3}^{\prime}=u\left(a_{3}\right)$ definiert.

Bemerkung 3.3.27. Über beliebigen Ringen hingegen sind Oktavenalgebren im Allgemeinen nicht durch ihre Norm bestimmt, wie Gille in [Gil12] zeigt. In seinem Artikel konstruiert er eine Klasse von nicht isomorphen Oktavenalgebren mit isometrischen Normen. Die dabei auftretenden Ringe sind von Dimension 14.

Über lokalen Ringen wiederum sind Oktavenalgebren mit guter Reduktion durch ihre Norm eindeutig bestimmt, wie Bix in [Bix81, Lemma 1.1] zeigt.

\subsubsection{Das Zentrum einer Kompositionsalgebra}

Kompositionsalgebren über Ringen von konstantem Rang 1 oder von konstantem Rang 2 sind stets kommutativ und assoziativ. In Rang 4 und Rang 8 gilt zumindest über Integritätsbereichen für das Zentrum und für die Menge der Elemente, die mit allen anderen Elementen das Assoziativgesetz erfüllen, dasselbe wie für Kompositionsalgebren über Körpern:

Lemma 3.3.28. Sei C eine Quaternionen- oder Oktavenalgebra über einem Integritätsbereich $R$. Dann ist das Zentrum von $C$ genau der Grundring $R$, d.h. $C$ ist zentral über $R$.

Ist $C$ eine Oktavenalgebra über dem Integritätsbereich $R$, so gilt für ein Element $x \in C$ mit

$$
(x y) z=x(y z) \text { für alle } y, z \in C
$$

ebenfalls $x \in R$.

Beweis. Sei $x \in Z(C)$ ein Element des Zentrums und sei $k$ der Quotientenkörper von $R$. Dann liegt $x$ offensichtlich auch im Zentrum der Skalarerweiterung $C_{k}=k \otimes_{R} C$. Als Quaternionen- oder Oktavenalgebra über einem Körper ist $C_{k}$ zentral, siehe [SV00, Prop. 1.9.1]. Somit gilt $x \in k e \cap C$, und da $C$ projektiv und also torsionsfrei ist, gilt $k e \cap C=R e$.

Ebenso erhalten wir die zweite Aussage aus dem entsprechenden Ergebnis über Körpern, siehe [SV00, Prop. 1.9.2].

Auch über (beliebigen kommutativen) Ringen ist die Bedingung (3.8) äquivalent $\mathrm{zu}(y x) z=y(x z)$ für alle $y, z \in C$ sowie äquivalent $\mathrm{zu}(y z) x=y(z x)$ für alle $y, z \in C$.

\subsubsection{Kompositionsalgebren über speziellen Körpern}

Wie wir bereits gesehen haben, ist ein Körper $k$ genau dann eine Kompositionsalgebra über sich selbst, wenn char $k \neq 2$ ist.

Eine quadratische Kompositionsalgebra über einem Körper $k$ ist entweder eine quadratische Körpererweiterung von $k$ oder isomorph zu $k \oplus k$ (und zerfällt). 
Aus Satz 3.3.26 folgt insbesondere auch, dass sich die Isomorphieklassen von Kompositionsalgebren über Körpern durch die Äquivalenzklassen von gewissen quadratischen Formen bestimmen lassen.

Eine Klassifikation der Quaternionen- und Oktavenalgebren über speziellen Körpern findet sich bei Springer und Veldkamp [SV00, Abschnitt 1.10]. Unter anderem zeigen sie:

1. Über algebraisch abgeschlossenen Körpern zerfallen alle Kompositionsalgebren und sind daher isomorph.

2. Über den reellen Zahlen $\mathbb{R}$ gibt es in Dimension 4 und 8 jeweils die zerfallende Kompositionsalgebra und je eine Kompositionsdivisionsalgebra, die Hamiltonschen Quaternionen bzw. die sogenannten Cayley-Zahlen.

3. Über endlichen Körpern gibt es in Dimension 4 und 8 jeweils nur die zerfallende Kompositionsalgebra.

4. Über einem vollständigen diskret bewerteten Körper mit endlichem Restklassenkörper gibt es in Dimension 4 die zerfallende Algebra und genau eine Isomorphieklasse einer Quaternionendivisionsalgebra und in Dimension 8 nur die zerfallende Kompositionsalgebra. Letzteres gilt, da jede quadratische Form eines Vektorraumes von Dimension $n \geq 5$ die 0 darstellt.

\subsection{Weitere Untersuchungen von Kompositionsalgebren}

\subsubsection{Kompositionsalgebren über Produktringen}

In diesem Abschnitt wollen wir zeigen, dass der Rang einer Kompositionsalgebra über einem beliebigen Ring nicht global konstant sein muss.

Sei $R=R_{1} \times R_{2}$ der Produktring zweier Ringe mit komponentenweiser Addition und Multiplikation. Dann ist sein Spektrum nicht zusammenhängend, siehe Anhang A.6.

Lemma 3.4.1. Seien $\left(C_{1}, N_{1}\right)$ eine Kompositionsalgebra über dem Ring $R_{1}$ und $\left(C_{2}, N_{2}\right)$ eine Kompositionsalgebra über dem Ring $R_{2}$. Dann ist das Produkt $C=C_{1} \times C_{2}$ mit der quadratischen Form $N\left(\left(x_{1}, x_{2}\right)\right)=\left(N_{1}\left(x_{1}\right), N_{2}\left(x_{2}\right)\right)$ eine Kompositionsalgebra über dem Produktring $R=R_{1} \times R_{2}$.

Beweis. Für $\lambda=\left(\lambda_{1}, \lambda_{2}\right) \in R$ und $x=\left(x_{1}, x_{2}\right), y=\left(y_{1}, y_{2}\right) \in C$ gilt

$$
N(\lambda x)=\left(N_{1}\left(\lambda_{1} x_{1}\right), N_{2}\left(\lambda_{2} x_{2}\right)\right)=\left(\lambda_{1}^{2} N_{1}\left(x_{1}\right), \lambda_{2}^{2} N_{2}\left(x_{2}\right)\right)=\lambda^{2} N(x)
$$

und

$$
\langle x, y\rangle=N(x+y)-N(x)-N(y)=\left(\left\langle x_{1}, y_{1}\right\rangle_{1},\left\langle x_{2}, y_{2}\right\rangle_{2}\right) .
$$


Da Letzteres offensichtlich eine Bilinearform ist, definiert das angegebene $N$ in der Tat eine quadratische Form auf $C$.

Da $N$ und die Multiplikation auf $C$ komponentenweise definiert sind, folgt aus der Multiplikativität der Normen $N_{i}$ auch die Multiplikativität der Norm $N$. Ebenso folgt aus der komponentenweisen Definition der Bilinearform und aus der Nichtausgeartetheit von $\left(C_{1}, N_{1}\right)$ und $\left(C_{2}, N_{2}\right)$, dass auch $(C, N)$ nichtausgeartet ist.

Da die $C_{i}$ treue $R_{i}$-Algebren sind, sind die Abbildungen $R_{i} \rightarrow C_{i}, \lambda_{i} \mapsto \lambda_{i} \cdot 1_{C_{i}}$ injektiv. Dann ist auch die Abbildung $R \rightarrow C,\left(\lambda_{1}, \lambda_{2}\right) \mapsto\left(\lambda_{1} \cdot 1_{C_{1}}, \lambda_{2} \cdot 1_{C_{2}}\right)$ injektiv, und somit ist $C$ treu über $R$.

Da jedes der beiden $C_{i}$ ein endlich erzeugter projektiver $R_{i}$-Modul ist, existiert jeweils ein $R_{i}$-Modul $Q_{i}$ mit $C_{i} \oplus Q_{i}=R_{i}^{n_{i}}$ für $i=1,2$. Ohne Einschränkung sei $n_{1}=n_{2}=n$. Dann ist $\left(C_{1} \times C_{2}\right) \oplus\left(Q_{1} \times Q_{2}\right)=R^{n}$, also ist auch $C$ endlich erzeugt und projektiv.

Die Einheiten des Produktringes setzen sich aus den Einheiten der Einzelringe zusammen: $R^{\times}=R_{1}^{\times} \times R_{2}^{\times}$.

Die maximalen Ideale von $R=R_{1} \times R_{2}$ sind gerade die Teilmengen der Form $R_{1} \times \mathfrak{m}_{2}$ und $\mathfrak{m}_{1} \times R_{2}$ für die maximalen Ideale $\mathfrak{m}_{1} \subset R_{1}$ und $\mathfrak{m}_{2} \subset R_{2}$.

Sei $\mathfrak{m}_{1} \times R_{2}$ ein solches maximales Ideal. Da $R \backslash\left(\mathfrak{m}_{1} \times R_{2}\right)=\left(R_{1} \backslash \mathfrak{m}_{1}\right) \times R_{2}$ gilt, ist die Lokalisierung von $R$ oder $C$ in diesem Ideal dann

$$
\begin{aligned}
& R_{\mathfrak{m}_{1} \times R_{2}}=\left(R_{1}\right)_{\mathfrak{m}_{1}} \times 0 \cong\left(R_{1}\right)_{\mathfrak{m}_{1}}, \\
& C_{\mathfrak{m}_{1} \times R_{2}}=\left(C_{1}\right)_{\mathfrak{m}_{1}} \times 0 \cong\left(C_{1}\right)_{\mathfrak{m}_{1}}
\end{aligned}
$$

Insbesondere gilt für den Rang von $C=C_{1} \times C_{2}$ in diesem maximalen Ideal $\mathfrak{m}_{1} \times R_{2}$ dann

$$
\operatorname{rg}_{\mathfrak{m}_{1} \times R_{2}}\left(C_{1} \times C_{2}\right)=\operatorname{rg}_{\mathfrak{m}_{1}}\left(C_{1}\right) .
$$

Korollar 3.4.2. Der Rang einer Kompositionsalgebra über einem nicht zusammenhängenden Ring muss nicht notwendigerweise konstant sein.

Die Quotienten von $R$ oder $C$ modulo einem maximalen Ideal $\mathfrak{m}_{1} \times R_{2}$ sind

$$
\begin{aligned}
R /\left(\mathfrak{m}_{1} \times R_{2}\right) & =R_{1} / \mathfrak{m}_{1} \times 0 \cong R_{1} / \mathfrak{m}_{1}, \\
C /\left(\mathfrak{m}_{1} \times R_{2}\right) C & =C_{1} / \mathfrak{m}_{1} C_{1} \times 0 \cong C_{1} / \mathfrak{m}_{1} C_{1} .
\end{aligned}
$$

Bemerkung 3.4.3. Beispielsweise sei $\mathbb{R} \times \mathbb{R}$ der Produktring der reellen Zahlen mit sich selbst. Seine beiden maximalen Ideale sind dann $0 \times \mathbb{R}$ und $\mathbb{R} \times 0$. Sei $C$ die Cayley-Oktavenalgebra über den reellen Zahlen $\mathbb{R}$. Dann ist die Produktalgebra $C \times \mathbb{R}$ eine Kompositionsalgebra über $\mathbb{R} \times \mathbb{R}$ und sie hat Rang 8 in dem maximalen Ideal $0 \times \mathbb{R}$ und Rang 1 in $\mathbb{R} \times 0$.

Außerdem kann $C \times \mathbb{R}$ offensichtlich nicht aus Verdopplung einer Unterkompositionsalgebra entstanden sein. 


\subsubsection{Unteralgebra zweier Elemente}

Sei $(C, N)$ eine Kompositionsalgebra über einem Ring $R$ und seien $x, y \in C$ zwei Elemente. Linearisieren der für alle Elemente $z \in C$ geltenden quadratischen Gleichung $z^{2}-\langle z, e\rangle z+N(z) e=0$ liefert

$$
x y+y x-\langle x, e\rangle y+\langle y, e\rangle x+\langle x, y\rangle e=0 .
$$

Nach Artins Theorem (siehe Satz 3.3.13) ist die von $x$ und $y$ erzeugte Unteralgebra von $C$ assoziativ, sodass zusammen mit der obigen Formel

$$
R[x, y]=R e+R x+R y+R x y
$$

folgt. Im Allgemeinen ist weder die Summe direkt noch $R[x, y]$ eine Unterkompositionsalgebra.

Lemma 3.4.4. Sei $(C, N)$ eine Kompositionsalgebra über $R$, und sei $x \in$ $C$. Dann ist $R[x]$ ein kommutativer Ring und $C$ kann als $R[x]$-Linksmodul aufgefasst werden via a.y $=$ ay für $a \in R[x], y \in C$.

BeweIs. Die Eins in $R[x]$ ist das Einselement $e$ aus $C$ und offensichtlich gilt $e . y=y$ für jedes $y \in C$. Sind $a, b \in R[x]$ und $y \in C$, so liegen alle drei Elemente in der von $x$ und $y$ erzeugten assoziativen Unteralgebra $R[x, y]$, und damit gilt $(a b) \cdot y=a \cdot(b . y)$.

Liegt $x$ nicht im Zentrum von $C$, so ist $C$ allerdings keine $R[x]$-Algebra.

\subsubsection{Operiert eine Quaternionenunteralgebra auf einer Oktavenalgebra?}

Sei die Oktavenalgebra $C$ als Verdopplung der Quaternionenalgebra $A$ über dem Ring $R$ gegeben. Es gebe also ein zu $A$ orthogonales Element $v \in A^{\perp}$ mit invertierbarer Norm $N(v)=-\lambda \in R^{\times}$so, dass $C=A \oplus A v$ gilt und die Multiplikation auf $C$ durch

$$
(a+b v)(c+d v)=(a c+\lambda \bar{d} b)+(d a+b \bar{c}) v
$$

für $a, b, c, d \in A$ gegeben ist.

Wir untersuchen zuerst, ob die gewöhnliche Multiplikation eine Linksoperation der Quaternionenunteralgebra $A$ auf $C$ erzeugt. Seien $a, c \in A$ und $x+y v \in C$ (mit $x, y \in A$ ), dann gilt

$$
\begin{aligned}
a(c(x+y v)) & =a c x+(y c a) v, \\
(a \cdot c)(x+y v) & =a c x+(y a c) v .
\end{aligned}
$$

Gleichheit der beiden linken Ausdrücke gilt folglich genau dann für alle Elemente $x+y v \in C$, wenn $a$ und $c$ miteinander kommutieren. Da die Quaternionenalgebra jedoch nicht kommutativ ist, operiert sie durch die in $C$ 
gegebene Multiplikation nicht auf der Oktavenalgebra. Insbesondere stellt die Linksmultiplikation von $A$ auf dem direktem Summanden $A v$ keine Operation dar.

Wir können jedoch eine getwistete Linksoperation von $A$ auf $A v$ definieren. Es sei

$$
a \cdot(d v):=\bar{a}(0+d v)=(d \bar{a}) v
$$

für $a \in A, d v \in A v$. Dann ist nämlich $e .(d v)=d v$ und

$$
a .(c .(d v))=(d \bar{c} \bar{a}) v=(d \overline{a c}) v=(a c) .(d v)
$$

für $a, c \in A, d v \in A v$. Entsprechend könnten wir auch eine getwistete Rechtsoperation von $A$ auf $A v$ via $(d v) . a:=(d v) \bar{a}=(d a) v$ für $a \in A, d v \in A v$ definieren. Allerdings sind die beiden so definierten Links- und Rechtsoperationen nicht miteinander verträglich, denn es ist

$$
\begin{aligned}
& (a \cdot(d v)) \cdot c=((d \bar{a}) v) \cdot c=(d \bar{a} c) v, \\
& a \cdot((d v) \cdot c)=a \cdot((d c) v)=(d c \bar{a}) v
\end{aligned}
$$

für $a, c \in C, d v \in A v$. Gleichheit gilt wiederum genau dann, wenn $a$ und $c$ miteinander kommutieren, also nicht im Allgemeinen.

Auch offensichtlich ist, dass $A$ auf diese getwistete Art nicht von links auf sich selbst, d. h. via $A \times A \rightarrow A,(a, b) \mapsto \bar{a} b$, operiert. Wir erhalten daher erneut keine Operation der Quaternionenunteralgebra auf der gesamten Oktavenalgebra.

Jedoch gilt für die getwistete Operation von $A$ auf $A v$, dass sie eine gewisse Komposition erlaubt: Es sind $(A, N)$ und $(A v, N)$ jeweils mit der auf $A$ bzw. auf $A v$ eingeschränkten Norm $N$ nichtausgeartete quadratische Moduln und es gilt

$$
N(a . d v)=N(a) N(d v)
$$

für alle $a \in A, d v \in A v$.

Die Zerlegung von $C$ in $A \oplus A v$ ausnutzend können wir aber in der Tat via

$$
a \cdot(x+y v)=a x+(a y) v
$$

für $a \in A$ und $x+y v \in C$ (mit $x, y \in A$ ) eine Operation von $A$ auf $C$ definieren. 



\section{Kapitel 4}

\section{Maximalordnungen in Kompositionsalgebren}

Sei in diesem Kapitel $R$ ein Integritätsbereich mit Quotientenkörper $K$.

Jede Kompositionsalgebra $C$ über $R$ erzeugt durch Skalarerweiterung eine Kompositionsalgebra $C_{K}$ über dem Quotientenkörper $K$. Wünschenswert wäre es, alle Kompositionsalgebren über $R$, die $C_{K}$ erzeugen, zu kennen. Zu diesem Zweck werden wir zunächst die allgemeineren sogenannten Ordnungen, insbesondere die Maximalordnungen von $C_{K}$ untersuchen, die wir in Analogie zu Ordnungen in assoziativen Azumaya-Algebren definieren.

\subsection{Gitter in quadratischen Räumen und in Kompositionsalgebren}

Definition. Ein Gitter in einem endlich-dimensionalen $K$-Vektorraum $V$ ist ein $R$-Untermodul $L$ von $V$, für den $K \cdot L=V$ gilt und der in einem endlich erzeugten $R$-Untermodul von $V$ enthalten ist.

Bemerkung 4.1.1. Sei $e_{1}, \ldots, e_{n}$ eine Vektorraumbasis von $V$ über $K$. Dann ist ein $R$-Untermodul $L$ von $V$ genau dann ein Gitter in $V$, wenn zwei Einheiten $a, b \in K^{\times}$mit

$$
a \sum_{i=1}^{n} R e_{i} \subseteq L \subseteq b \sum_{i=1}^{n} R e_{i}
$$

existieren. Die obige Definition zeigt, dass die Existenz geeigneter $a$ und $b$ unabhängig von der Wahl der Basis ist.

Insbesondere ist jeder endlich erzeugte $R$-Untermodul $L$ von $V$, für den $K \cdot L=$ $V$ gilt, ein Gitter in $V$. Ist der Ring $R$ noethersch, so gilt auch umgekehrt, dass jedes Gitter endlich erzeugt ist. Ist $R$ sogar ein Hauptidealring, so ist jedes Gitter endlich erzeugt und frei. 
Definition. Sei $(V, q)$ ein endlich-dimensionaler $K$-Vektorraum mit quadratischer Form. Ein ganzes Gitter in $(V, q)$ ist ein Gitter $L$, für das $q(L) \subseteq R$ gilt. Ein ganzes Gitter $L$ heißt maximal, wenn es in keinem ganzen Gitter echt enthalten ist.

Ein ganzes Gitter $L$ in $(V, q)$ ist mit der Einschränkung der quadratischen Form ein quadratischer $R$-Modul.

$\mathrm{Zu}$ einem Gitter $L$ in $(V, q)$ betrachten wir die dualen Mengen

$$
\begin{aligned}
L^{\#} & =\{x \in V \mid\langle x, y\rangle \in R \text { für alle } y \in L\}, \\
L^{(\#)} & =\{x \in V \mid\langle x, y\rangle \in R \text { für alle } y \in L \text { und } q(x) \in R\} .
\end{aligned}
$$

Die Bezeichnung $L^{(\#)}$ für die Menge $L^{\#} \cap\{x \in V \mid q(x) \in R\}$ haben wir für diese Arbeit neu eingeführt (und werden sie nur sparsam verwenden).

Ist $(V, q)$ nichtausgeartet, so ist $L^{\#}$ ein Gitter und wird das duale Gitter $z u$ $L$ genannt. Zum Beispiel ist $\left(a \sum R e_{i}\right)^{\#}=a^{-1} \sum R e_{i}^{*}$, wobei $\left\{e_{i}^{*}\right\}$ die duale Basis mit $\left\langle e_{j}, e_{i}^{*}\right\rangle=\delta_{i j}$ bezeichne, siehe etwa [Kne02, (14.2)].

Sind $L_{1} \subseteq L_{2}$ zwei Gitter, so gilt $L_{1}^{\#} \supseteq L_{2}^{\#}$ und $L_{1}^{(\#)} \supseteq L_{2}^{(\#)}$. Ist $L$ ein ganzes Gitter in $(V, q)$, so gilt $L \subseteq L^{(\#)} \subseteq L^{\#}$. Ein Gitter $L$ heißt selbstdual, wenn $L=L^{\#}$ gilt. Ist $2 \in R^{\times}$invertierbar, so ist jedes selbstduale Gitter ganz.

Lemma 4.1.2. Sei $(V, q)$ ein endlich-dimensionaler quadratischer Vektorraum über $K=\operatorname{Quot}(R)$. Ein Gitter $L$ in $(V, q)$ ist genau dann maximal, wenn $L=L^{\#} \cap\{x \in V \mid q(x) \in R\}$, also wenn $L=L^{(\#)}$ gilt.

Beweis. Sei $L=L^{(\#)}$. Dann ist $L$ ganz. Ist $L_{2} \supseteq L$ ein ganzes Gitter, so gilt $L \subseteq L_{2} \subseteq L_{2}^{(\#)} \subseteq L^{(\#)}$ und daher $L=L_{2}$. Folglich ist $L$ maximal.

Ist umgekehrt $x \in L^{(\#)} \backslash L$, so ist $L+R x$ ein Gitter, das $L$ echt enthält und das wegen $L+R x \subseteq L^{(\#)}$ ebenfalls ein ganzes Gitter ist.

Definition. Sei $(C, N)$ eine Kompositionsalgebra über $K=\operatorname{Quot}(R)$. Ein Element $x \in C$ heißt ganz über $R$, wenn seine Norm $N(x)$ und seine Spur $\langle x, e\rangle$ in $R$ liegen.

Jedes ganze Element $x$ ist also Nullstelle eines normierten Polynoms mit Koeffizienten aus $R$, nämlich von $T^{2}-\langle x, e\rangle T+N(x) \in R[T]$. Außerdem ist $R[x]$ eine kommutative und assoziative Algebra über $R$. Folglich ist $x$ insbesondere ein ganzes Element im Sinne der kommutativen Algebra, d. h. es ist Nullstelle eines nichttrivialen, normierten Polynoms beliebigen Grades mit Koeffizienten in $R$.

Durch die Beschränkung auf quadratische Polynome haben wir den Begriff der Ganzheit in Kompositionsalgebren strenger gefasst als in der kommutativen Algebra. Es gilt: 
Lemma 4.1.3. Ein Element $x$ einer Kompositionsalgebra $(C, N)$ über $K=$ $\operatorname{Quot}(R)$ ist genau dann Nullstelle eines normierten Polynoms $h \neq 0$ mit Koeffizienten aus $R$, wenn $N(x)$ und $\langle x, e\rangle$ ganz über $R$ sind.

Beweis. Es sei $f=T^{2}-\langle x, e\rangle T+N(x) \in K[T]$ das Polynom zur quadratischen Gleichung von $x$. Insbesondere gilt also $f(x)=0$.

Seien $N(x)$ und $\langle x, e\rangle$ ganz über $R$. Dann ist $S=R[N(x),\langle x, e\rangle]$ ganz über $R$, und da die Koeffizienten von $f$ in $S$ liegen, ist die kommutative und assoziative Algebra $S[x] \subseteq C$ ganz über $S$. Damit ist $S[x]$ auch ganz über $R$ und $x$ ist Nullstelle eines normierten Polynoms $h \neq 0$ mit Koeffizienten aus $R$.

Sei umgekehrt $x$ Nullstelle eines normierten Polynoms $h \neq 0$ mit Koeffizienten aus $R$. Division mit Rest von $h$ durch $f$ in $K[T]$ liefert eine Zerlegung $h=f g+r$ mit Polynomen $g, r \in K[T] \operatorname{mit} \operatorname{grad}(r)<2$. Es ist $r(x)=0$. Ist $\operatorname{grad}(r)=1$, so ist $x=\lambda e$ mit einem $\lambda \in K$ und $\lambda$ ist ebenfalls Nullstelle von $h$ und somit ganz über $R$. Damit sind auch $N(x)=\lambda^{2}$ und $\langle x, e\rangle=2 \lambda$ ganz über $R$. Ist $\operatorname{grad}(r)<1$, so gilt $h=f g$, da $r(x)=0$ ist. Sei $f=\left(T-\alpha_{1}\right)\left(T-\alpha_{2}\right)$ über einem Zerfällungskörper von $K$. Dann sind $\alpha_{1}$ und $\alpha_{2}$ auch Nullstellen von $h$ und somit ganz über $R$. Damit sind auch $N(x)=\alpha_{1} \alpha_{2}$ und $\langle x, e\rangle=\alpha_{1}+\alpha_{2}$ ganz über $R$.

Über ganzabgeschlossenen Integritätsbereichen stimmen die beiden Varianten der Ganzheit daher überein:

Korollar 4.1.4. Sei $R$ ein ganzabgeschlossener Integritätsbereich und $(C, N)$ eine Kompositionsalgebra über $K=\operatorname{Quot}(R)$. Dann ist ein Element $x \in C$ genau dann ganz in der Kompositionsalgebra, wenn es auch im Sinne der kommutativen Algebra ganz über $R$ ist.

Ein Gitter in einer Kompositionsalgebra, dessen Elemente ganz über $R$ sind, ist offensichtlich ein ganzes Gitter. Umgekehrt gilt Folgendes:

Lemma 4.1.5. Ist $L$ ein ganzes Gitter in einer Kompositionsalgebra $(C, N)$ über $K=\operatorname{Quot}(R)$, das e enthält, so sind alle Elemente von $L$ ganz über $R$. Ist $L$ ein Gitter in $(C, N)$, dessen Elemente ganz sind, so ist auch $L+R e$ ein Gitter, dessen Elemente ganz sind. Insbesondere enthält jedes maximale Gitter, dessen Elemente ganz sind, die Eins e.

Beweis. Sei $L$ ein ganzes Gitter, das $e$ enthält, und sei $x \in L$. Mit $e$ liegt dann auch $x+e$ in $L$ und es gilt $N(x), N(x+e) \in R$. Somit ist $\langle x, e\rangle=$ $N(x+e)-N(x)-N(e) \in R$ und folglich ist $x$ ganz über $R$.

Mit $L$ ist auch $L+R e$ ein $R$-Gitter in $V$. Seien nun alle Elemente aus $L$ ganz. Dann gilt $N(x+\lambda e)=N(x)+\lambda^{2}+\lambda\langle x, e\rangle \in R$ für $x \in L, \lambda \in R$, und die zweite Aussage folgt aus der ersten. 


\subsection{Maximalordnungen}

Definition. Sei $(C, N)$ eine Kompositionsalgebra über $K=\operatorname{Quot}(R)$. Eine Ordnung in $(C, N)$ ist eine $R$-Unteralgebra von $C$, die die Eins aus $C$ enthält und die als $R$-Modul ein ganzes Gitter in $(C, N)$ ist.

Eine Maximalordnung in $(C, N)$ ist eine Ordnung, die in keiner Ordnung echt enthalten ist.

Korollar 4.2.1. Die Elemente einer Ordnung in einer Kompositionsalgebra sind ganz.

BeweIs. Eine Ordnung ist ein ganzes Gitter, das $e$ enthält. Damit folgt die Behauptung aus Lemma 4.1.5.

Wir verlangen, dass eine Ordnung per definitionem ein ganzes Gitter ist, also nur ganze Elemente enthält. In assoziativen Algebren wird meist allgemeiner eine Ordnung als beliebiges multiplikativ abgeschlossenes $R$-Gitter, das $e$ enthält, definiert, siehe Abschnitt 2.3.1, Seite 16. Allerdings wird für die meisten weitergehenden Untersuchungen vorausgesetzt, dass der Grundring $R$ nicht nur noethersch, sondern auch ganzabgeschlossen ist. Siehe hierzu etwa Reiner ([Rei75, Ch.2, Seite 108]) oder Artin und de Jong ([AdJ, Ch. 1.2]) Letztere begründen auch, warum es sinnvoll ist, sich auf ganzabgeschlossene Ringe zu beschränken. Über ganzabgeschlossenen Ringen gilt:

Lemma 4.2.2. Sei $R$ ein ganzabgeschlossener Integritätsbereich und sei $(C, N)$ eine Kompositionsalgebra über $K=\operatorname{Quot}(R)$. Dann ist jedes Element eines multiplikativ abgeschlossenen Gitters $L$ in $(C, N)$ ganz. Insbesondere ist dann jedes multiplikativ abgeschlossene Gitter, das die Eins enthält, eine Ordnung.

Beweis. Mit $L$ ist auch $L+R e$ ein multiplikativ abgeschlossenes Gitter in $(C, N)$ und daher in einem endlich erzeugten $R$-Modul $M$ enthalten. Insbesondere enthält $M$ für jedes $x \in L$ die von $x$ erzeugte Unteralgebra $R[x]$. Daher ist $x$ ganz über $R$ im Sinne der kommutativen Algebra und genügt einer normierten ganzen Polynomgleichung (beliebigen Grades). Aus Korollar 4.1.4 folgt, dass $x$ ganz über $R$ ist.

Über ganzabgeschlossenen Ringen, unter anderem also über diskreten Bewertungsringen und über Hauptidealringen, unterscheiden sich die beiden Definitionen von Ordnungen in Kompositionsalgebren und Ordnungen in assoziativen Algebren somit nicht. Insbesondere stimmen dann auch die Definitionen von Maximalordnungen überein.

Ordnungen lassen sich mit Hilfe von Gittern konstruieren: Ist $L$ ein ganzes Gitter einer Kompositionsalgebra, das die Eins enthält, so liefert das unten folgende Lemma 4.2.5 eine Ordnung, die in $L$ enthalten ist. In Lemma 4.2.6 werden hinreichende Bedingungen angegeben, unter denen ein Abstieg einer 
Ordnung über einer Ringerweiterung - analog zum assoziativen Fall bei Reiner [Rei75, Ch. 2.8, Seite 109] - möglich ist. Wir beginnen mit einem Hilfslemma:

Lemma 4.2.3. Der Durchschnitt zweier Gitter ist ein Gitter.

Beweis. Seien $L_{1}$ und $L_{2}$ zwei $R$-Gitter in einem quadratischen Vektorraum $(V, q)$ über $K=\operatorname{Quot}(R)$. Der Durchschnitt zweier $R$-Moduln ist ein $R$-Modul. Als Gitter ist $L_{1}$ in einem endlichen erzeugten $R$-Modul $M$ enthalten; dieser enthält auch den Durchschnitt $L_{1} \cap L_{2}$. Sei $x \in V$. Dann existieren Elemente $x_{1} \in L_{1}$ und $x_{2} \in L_{2}$ und Skalare $r_{1}, r_{2}, s_{1}, s_{2} \in R$ mit $x=\frac{r_{1}}{s_{1}} x_{1}=\frac{r_{2}}{s_{2}} x_{2}$. Es liegt $\left(s_{1} s_{2}\right) x$ im Durchschnitt $L_{1} \cap L_{2}$ und $s_{1} s_{2}$ in $K^{\times}$. Daher ist $V=K\left(L_{1} \cap L_{2}\right)$, und folglich ist $L_{1} \cap L_{2}$ ein Gitter.

Korollar 4.2.4. Der Durchschnitt zweier ganzer Gitter ist ein ganzes Gitter.

BEWEIS. Offensichtlich ist jedes Untergitter eines ganzen Gitters ganz.

Lemma 4.2.5. Sei L ein Gitter in einer Kompositionsalgebra $(C, N)$ über $K=$ $\operatorname{Quot}(R)$. Dann sind

$$
O_{r}(L)=\{x \in C \mid L x \subseteq L\} \quad \text { und } \quad O_{\ell}(L)=\{x \in C \mid x L \subseteq L\}
$$

ebenfalls Gitter in C. Ihr Durchschnitt $O_{r}(L) \cap O_{\ell}(L)$ ist ein multiplikativ abgeschlossenes Gitter, das die Eins enthält. Ist $C$ assoziativ, so sind auch $O_{r}(L)$ und $O_{\ell}(L)$ selbst multiplikativ abgeschlossen.

In einer assoziativen Algebra bezeichnen $O_{r}(L)$ und $O_{\ell}(L)$ die sogenannte Rechts- bzw. Linksordnung des Gitters L.

Beweis. Da $L$ ein Gitter ist, existieren zur Eins $e \in C$ Elemente $r, s \in R, s \neq 0$ mit $e \in \frac{r}{s} L$, insbesondere ist dann $s e \in L$. Für jedes $x \in O_{r}(L)$ gilt daher $s x=(s e) x \in L$, also ist $O_{r}(L) \subseteq s^{-1} L$. Sei $x \in C$. Als Gitter ist $L$ in einem endlich erzeugten $R$-Modul $M=\sum R e_{i}$ enthalten. $\mathrm{Zu} e_{i} x \in C$ gibt es Elemente $r_{i}, s_{i} \in R$ mit $e_{i} x \in \frac{r_{i}}{s_{i}} L$. Sei $t \neq 0$ das Produkt der Nenner $s_{i}$, dann gilt $e_{i}(t x) \in L$ und damit $L(t x) \subseteq M(t x) \subseteq L$. Es folgt $t x \in O_{r}(L)$. Also gilt $C=K \cdot O_{r}(L)$, und folglich ist $O_{r}(L)$ ein Gitter in $C$. Offensichtlich ist dann auch $O_{\ell}(L)$ ein Gitter in $C$ und das Einselement $e$ ist in beiden Mengen enthalten. Als Durchschnitt zweier Gitter ist auch $O_{r}(L) \cap O_{\ell}(L)$ ein Gitter in $C$.

Seien $x, y \in O_{r}(L) \cap O_{\ell}(L)$ und sei $\ell \in L$. Dann gilt

$$
\ell(x y)=-\{\ell, x, y\}+(\ell x) y=\{x, \ell, y\}+(\ell x) y=(x \ell) y-x(\ell y)+(\ell x) y,
$$

wobei $\{a, b, c\}=(a b) c-a(b c)$ den Assoziator der Elemente $a, b, c \in C$ bezeichne. Die zweite Gleichheit gilt, da der Assoziator eine alternierende Funktion ist. Die drei Summanden der rechten Seite liegen in $L$, daher ist auch $(x y)$ ein Element aus $O_{r}(L)$. Ebenso folgt, dass $(x y)$ auch in $O_{\ell}(L)$ liegt. Damit ist $O_{r}(L) \cap O_{\ell}(L)$ multiplikativ abgeschlossen. 
Die Multiplikativität von $O_{r}(L)$ und $O_{\ell}(L)$ in assoziativen Algebren ist offensichtlich.

Lemma 4.2.6. Sei $R^{\prime}$ eine Ringerweiterung von $R$, die in $K=\operatorname{Quot}(R)$ enthalten ist, und seien $(V, q)$ ein quadratischer Modul und $(C, N)$ eine Kompositionsalgebra über $K$. Dann gilt:

1. Sei $L^{\prime}$ ein endlich erzeugtes $R^{\prime}$-Gitter in $(V, q)$. Dann existiert ein endlich erzeugtes $R$-Gitter $L$ mit $R^{\prime} L=L^{\prime}$.

2. Sei $R^{\prime}=S^{-1} R$ mit einer multiplikativen Menge $S \subset R$ und sei $L^{\prime}$ ein beliebiges $R^{\prime}$-Gitter. Dann existiert ein $R$-Gitter $L$ mit $R^{\prime} L=L^{\prime}$. Ist $L^{\prime}$ endlich erzeugt und ganz, so kann auch $L$ endlich erzeugt und ganz gewählt werden.

3. Sei $R^{\prime}=S^{-1} R$ und sei $B^{\prime}$ eine endlich erzeugte $R^{\prime}$-Ordnung in $(C, N)$. Dann existiert eine $R$-Ordnung $B$ mit $R^{\prime} B=B^{\prime}$.

BewEIS. Ist $L^{\prime}=\sum R^{\prime} e_{i}$ ein endlich erzeugtes $R^{\prime}$-Gitter, so ist offensichtlich $L=\sum R e_{i}$ ein endlich erzeugtes $R$-Gitter mit $L^{\prime}=R^{\prime} L$.

Sei nun $R^{\prime}=S^{-1} R$. Ist $L^{\prime}$ ein beliebiges $R^{\prime}$-Gitter, so existiert ein endlich erzeugtes $R^{\prime}$-Gitter $M^{\prime}$ und ein $a \in R, a \neq 0$ mit $a M^{\prime} \subseteq L^{\prime} \subseteq M^{\prime}$. Sei $M$ ein endlich erzeugtes $R$-Gitter mit $R^{\prime} M=M^{\prime}$. Dann ist $L=L^{\prime} \cap M$ ein $R$-Modul, für den $a M \subseteq L \subseteq M$ gilt. Folglich ist $L$ ein $R$-Gitter. Sei $x \in L^{\prime}$ und sei $e_{1}, \ldots, e_{m}$ ein Erzeugendensystem von $M$ über $R$. Dann existieren Skalare $r_{i}^{\prime} \in R^{\prime}$ mit $x=\sum_{i} r_{i}^{\prime} e_{i}$. Sei $s \in S$ das Produkt der Nenner der $r_{i}^{\prime}$. Dann gilt $s \in R^{\prime \times}$ und $s x \in L$, mithin liegt $x$ in $R^{\prime} L$.

Ist $L^{\prime}=\sum R^{\prime} e_{i}$ endlich erzeugt und ganz, so sei $s \in S$ das Produkt der Nenner der $q\left(e_{i}\right)$ und der Werte $\left\langle e_{i}, e_{j}\right\rangle=q\left(e_{i}+e_{j}\right)-q\left(e_{i}\right)-q\left(e_{j}\right) \in R^{\prime}=S^{-1} R$. Dann gilt $q\left(L^{\prime}\right) \subseteq s^{-1} R$ und $L=s L^{\prime}$ ist ein ganzes $R$-Gitter mit $R^{\prime} L=L^{\prime}$.

Sei $B^{\prime}$ eine endlich erzeugte $R^{\prime}$-Ordnung und sei $L$ ein ganzes, endlich erzeugtes $R$-Gitter mit $R^{\prime} L=B^{\prime}$, das o. B.d. A. die Eins $e$ enthalte (ansonsten ersetze $L$ durch $s L+R e)$. Dann ist $B=O_{r}(L) \cap O_{\ell}(L)$ ein multiplikativ abgeschlossenes, ganzes $R$-Gitter, das $e$ enthält, und somit eine $R$-Ordnung. Sei $x \in B^{\prime}$ und seien $e_{1}, \ldots, e_{m}$ Erzeuger von $L$ über $R$. Dann gilt $e_{i} x \in B^{\prime}$ und damit $e_{i} x=$ $r_{i}^{\prime} x_{i}$ für geeignete $r_{i}^{\prime} \in R^{\prime}$ und $x_{i} \in L$. Sei $t_{1} \in S$ das Produkt der Nenner der $r_{i}^{\prime}$. Dann gilt $L\left(t_{1} x\right) \subseteq L$ und somit liegt $t_{1} x$ in $O_{r}(L)$. Da auch $t_{1} x \in B^{\prime}$ gilt, existiert analog ein $t_{2} \in S$ mit $t_{2} t_{1} x \in O_{\ell}(L)$. Insgesamt gilt damit $x \in\left(t_{2} t_{1}\right)^{-1} O_{r}(L) \cap O_{\ell}(L)$.

Um die Bedingungen der Maximalordnungen weiter zu untersuchen, verallgemeinern wir zuerst einige Aussagen aus dem Verdopplungsverfahren von $B \mathrm{zu}$ $B \oplus B v$, indem wir die Voraussetzungen an $B$ und $v \in B^{\perp}$ mit $N(v) \in R^{\times}$ abschwächen und die direkte Summe durch die einfache Summe ersetzen. 
Lemma 4.2.7. Sei $B$ eine R-Unteralgebra einer Kompositionsalgebra $(C, N)$ über $K=\operatorname{Quot}(R)$, die die Eins e enthält und abgeschlossen unter Konjugation ist. Ist $v \in C$ ein Element, für das $N(v) \in R$ und $\langle v, x\rangle \in R$ für alle $x \in B$ gilt, so ist auch $B+B v$ eine $R$-Unteralgebra von $C$.

Sind alle Elemente aus $B$ ganz, so sind auch alle Elemente aus $B+B v$ ganz über $R$.

BeweIs (nach [vBS59, (3.2)]). Dass $B+B v$ ein $R$-Untermodul ist, der $e$ enthält, ist offensichtlich. Es bleibt zu zeigen, dass $(a+b v)(c+d v)$ für alle $a, b, c, d \in B$ wieder in $B+B v$ liegt.

Ausführliche Umformungen ergeben (unabhängig davon, ob $C$ eine Kompositionsalgebra über einem Körper oder einem beliebigen Ring ist)

$$
\begin{aligned}
a(d v)= & \langle v, e\rangle a d-\langle v, d\rangle a+\langle v, \bar{a}\rangle \bar{d}-\langle v, \overline{d a}\rangle e+(d a) v \\
(b v) c= & \langle v, \bar{c}\rangle b-\langle v, e\rangle b \bar{c}+(b \bar{c}) v \\
(b v)(d v)= & \langle v, e\rangle b(d v)-\langle v, b\rangle d v+\langle v, e\rangle v(\bar{b} d)-\langle v, \bar{b} d\rangle v \\
& +\langle v, e\rangle v(\bar{d} b)-N(v) \bar{d} b .
\end{aligned}
$$

Da $e \in B$ und $\langle v, x\rangle \in R$ für alle $x \in B$ gilt und da $B$ unter Multiplikation und Konjugation abgeschlossen ist, liegen offensichtlich alle Summanden von $a(d v)$ und $(b v) c$ in $B+B v$. Damit liegen aber auch alle Summanden von $(b v)(d v)$ in $B+B v$, und $B+B v$ ist somit multiplikativ abgeschlossen.

Sind alle Elemente aus $B$ ganz, so gilt $N(a+b v)=N(a)+N(b) N(v)+\langle a, b v\rangle=$ $N(a)+N(b) N(v)+\langle\bar{b} a, v\rangle \in R$ und ebenso $\langle a+b v, e\rangle=\langle a, e\rangle+\langle b v, e\rangle=$ $\langle a, e\rangle+\langle v, \bar{b}\rangle \in R$ für alle $a, b \in B$.

Um zu entscheiden, wann eine Ordnung maximal ist, reicht es, die Maximalität als Gitter zu prüfen:

Satz 4.2.8. Sei $B$ eine Ordnung in einer Kompositionsalgebra $(C, N)$. Dann ist $B$ genau dann eine Maximalordnung, wenn $B$ als Gitter maximal ist.

Beweis (nach [vBS59, (3.1)]). Ist $B$ als Gitter maximal, so ist es das auch als Ordnung.

Sei umgekehrt $B$ eine Maximalordnung, dann gilt insbesondere $e \in B$ und $\langle x, e\rangle \in R$ für alle $x \in B$, da die Elemente von Maximalordnungen ganz sind. Mit $x$ liegt dann auch $\bar{x}=\langle x, e\rangle e-x$ in $B$, folglich ist $B$ abgeschlossen unter Konjugation. Sei $L$ ein ganzes Gitter, das $B$ enthält. Für $v \in L$ gilt dann $N(v) \in R$ und $\langle v, x\rangle=N(v+x)-N(v)-N(x) \in R$ für alle $x \in B$. Nach Lemma 4.2.7 ist damit auch $B+B v$ eine Ordnung in $C$. Aus der Maximalität von $B$ folgt $v \in B$ und damit $L=B$.

Eine Ordnung ist ein ganzes Gitter, das multiplikativ abgeschlossen ist und die Eins enthält. In dem obigen Satz kann die Grundbedingung $e \in B$ durch $\langle x, e\rangle \in R$ für alle $x \in B$ ersetzt werden: 
Korollar 4.2.9. Ein Gitter, das nur ganze Elemente enthält und das multiplikativ abgeschlossen ist, ist genau dann eine Maximalordnung, wenn es als Gitter maximal ist.

BeweIs. Sei $L$ ein multiplikativ abgeschlossenes Gitter ganzer Elemente. Ist $L$ maximal, so enthält es nach Lemma 4.1.5 die Eins $e$, ist also insbesondere eine Ordnung.

Auch die Forderung, dass das Gitter multiplikativ abgeschlossen ist, kann man durch eine geeignete andere Bedingung ersetzen.

Lemma 4.2.10. Sei $B$ ein maximales Gitter einer Kompositionsalgebra $(C, N)$ über $K=\operatorname{Quot}(R)$. Gilt $e \in B$ und $\langle x y, z\rangle \in R$ für alle $x, y, z \in B$, so ist $B$ eine Maximalordnung in $(C, N)$.

BeweIs (nach [vBS59, (3.1)]). Seien $x, y \in B$. Für alle $\lambda \in R, z \in R$ gilt dann $N(\lambda x y+z)=\lambda^{2} N(x) N(y)+N(z)+\lambda\langle x y, z\rangle \in R$. Somit ist $B+R x y$ ein ganzes Gitter, und aus der Maximalität von $B$ folgt $x y \in B$.

Umgekehrt ist klar, dass für alle Elemente $x, y, z$ einer Maximalordnung $B$ auch $\langle x y, z\rangle=N(x y+z)-N(x y)-N(z)$ in $R$ liegt.

Die Ordnungen einer Kompositionsalgebra $(C, N)$ über $K=\operatorname{Quot}(R)$ sind in gewisser Weise eine Verallgemeinerung der Kompositionsalgebren über $R$, die $C$ erzeugen. Es bestehen die folgenden Zusammenhänge:

Lemma 4.2.11. Sei $(B, N)$ eine Kompositionsalgebra über $R$. Dann ist $B$ eine Ordnung in der Kompositionsalgebra $\left(B_{K}=K \otimes B, N_{K}\right)$ über dem Quotientenkörper $K=\operatorname{Quot}(R)$. Ist $(B, N)$ regulär, so ist $B$ eine Maximalordnung in $\left(B_{K}, N_{K}\right)$.

BEweIs. Als projektiver Modul über einem nullteilerfreien Ring ist $B$ torsionsfrei, und die kanonische Abbildung $B \rightarrow B_{K}, x \mapsto 1 \otimes x$ ist eine Inklusion. Folglich ist $B$ eine Ordnung in $B_{K}$.

Sei nun $x \in B^{\#}$. Dann ist $\varphi_{x}: B \rightarrow R, y \mapsto\langle x, y\rangle$ ein Element aus $B^{*}=$ $\operatorname{Hom}_{R}(B, R)$. Ist $B$ regulär, so gibt es ein $x^{\prime} \in B$ mit $\varphi_{x}(y)=\left\langle x^{\prime}, y\right\rangle$, also mit $\left\langle x-x^{\prime}, y\right\rangle=0$ für alle $y \in B$. Dann gilt aber auch $\left\langle x-x^{\prime}, y^{\prime}\right\rangle=0$ für alle $y^{\prime} \in B_{K}$ und somit $x-x^{\prime}=0$ bzw. $x=x^{\prime} \in B$. Damit ist $B=B^{\#}$ maximal als Gitter, folglich auch als Ordnung.

Bemerkung 4.2.12. Sei umgekehrt $B$ eine Ordnung in einer Kompositionsalgebra $(C, N)$ über $K$. Dann ist die Einschränkung von $N$ auf $B$ nichtausgeartet, denn: Sei $\langle x, z\rangle=0$ für alle $z \in B$; für jedes $z^{\prime} \in C$ existieren $\lambda \in K, z \in B$ mit $z^{\prime}=\lambda z$, sodass auch $\left\langle x, z^{\prime}\right\rangle=\lambda\langle x, z\rangle=0$ und damit insgesamt $x=0$ gilt. Außerdem gilt $N(x y)=N(x) N(y)$ für alle $x, y \in B$, und als Unteralgebra von $C$ ist auch $B$ treu. Damit erfüllt jede (Maximal-) Ordnung über $R$ die meisten Eigenschaften einer Kompositionsalgebra, ungewiss ist nur, ob sie auch endlich erzeugt und projektiv ist. 


\subsection{Maximalordnungen über Hauptidealringen}

Über einem Hauptidealring ist jedes Gitter endlich erzeugt und frei und somit insbesondere projektiv. Zusammen mit den obigen Überlegungen ergibt sich für Ordnungen damit die folgende Aussage:

Lemma 4.3.1. Sei $R$ ein Hauptidealring und $(C, N)$ eine Kompositionsalgebra über $K=\operatorname{Quot}(R)$. Dann ist jede Ordnung $D$ in $(C, N)$ eine Kompositionsalgebra über $R$.

Ist $(C, N)$ die zerfallende Oktavenalgebra über dem Quotientenkörper eines Hauptidealringes, so werden wir zeigen, dass alle Maximalordnungen in $C$ isomorph zueinander sind. Wir folgen dabei der Argumentation von van der Blij und Springer [vBS59], auch wenn diese sich in der Formulierung auf vollständige diskrete Bewertungsringe beschränken.

Ein Element $x$ eines Gitters $L$ heiße primitiv, wenn aus $x=\lambda x^{\prime}$ mit $\lambda \in R$ und $x^{\prime} \in L$ folgt, dass $\lambda$ eine Einheit in $R$ ist. Da hier $R$ ein Hauptidealring ist und $K L=V$ gilt, existiert zu jedem $z \in V, z \neq 0$ ein primitives $x \in L$ mit $z \in K x$. Außerdem bezeichne im Folgenden $\delta_{i j}$ das Kroneckersymbol mit $\delta_{i i}=1$ und $\delta_{i j}=0$ für $i \neq j$.

Lemma 4.3.2. Sei $R$ ein Hauptidealring, und sei $(V, q)$ ein hyperbolischer quadratischer Raum von Dimension $2 n$ über $K=\operatorname{Quot}(R)$. Sei $L$ ein maximales ganzes Gitter in $(V, q)$. Dann besitzt $L$ eine Basis $\left\{e_{i}, e_{-i} \mid i=1, \ldots, n\right\}$ über $R$ mit $q\left(e_{i}\right)=0$ und $\left\langle e_{i}, e_{j}\right\rangle=\delta_{i,-j}$ für alle $i, j \in\{ \pm 1, \ldots, \pm n\}$. Umgekehrt ist jedes Gitter mit einer solchen Basis maximal.

Beweis. Langfassung der Beweisskizze aus [vBS59, (3.3)]. - Sei $x \in V, x \neq 0$ ein isotropes Element. Dann existiert ein primitives $e_{n} \in L$ mit $x=\lambda e_{n}$ für ein $\lambda \in K^{\times}$. Da $q\left(e_{n}\right)=\lambda^{-2} q(x)=0$ gilt, ist auch $e_{n}$ isotrop.

Die Menge $I=\left\{\left\langle x, e_{n}\right\rangle \mid x \in L\right\}$ ist offensichtlich ein Ideal in $R$. Da $R$ ein Hauptidealring ist, existiert ein $a \in R$ mit $I=(a)$, und da $q$ nichtausgeartet ist, ist $a \neq 0$ in $K$ invertierbar. Das Gitter $L^{\prime}=L+R a^{-1} e_{n}$ ist ebenfalls ganz, denn für $x \in L, \lambda \in R$ gilt $q\left(x+\lambda a^{-1} e_{n}\right)=q(x)+0+a^{-1} \lambda\left\langle x, e_{n}\right\rangle \in R+a^{-1} I$ und es ist $a^{-1} I=a^{-1}(a)=R$. Aus der Maximalität von $L$ folgt $a^{-1} e_{n} \in L$ und aus der Primitivität von $e_{n}$ dann $a \in R^{\times}$. Damit existiert ein $x \in L$ mit $\left\langle x, e_{n}\right\rangle=1$. Sei $\lambda=q(x)$; für $e_{-n}:=x-\lambda e_{n}$ gilt dann $\left\langle e_{-n}, e_{n}\right\rangle=\left\langle x, e_{n}\right\rangle-2 \lambda q\left(e_{n}\right)=1$ und $q\left(e_{-n}\right)=q(x)+\lambda^{2} q\left(e_{n}\right)-\lambda\left\langle x, e_{n}\right\rangle=0$.

Der von $e_{n}, e_{-n}$ erzeugte Unterraum $R e_{n} \oplus R e_{-n}$ in $L$ ist regulär, sodass wir eine orthogonale Zerlegung $L=\left(R e_{n} \oplus R e_{-n}\right) \perp E$ mit $E=\left(R e_{n} \oplus R e_{-n}\right)^{\perp}$ erhalten. Für $x \in L$ ist diese durch $x=\left\langle x, e_{-n}\right\rangle e_{n}+\left\langle x, e_{n}\right\rangle e_{-n}+y$ gegeben, denn es gilt $\left\langle y, e_{n}\right\rangle=\left\langle x, e_{n}\right\rangle-\left\langle x, e_{-n}\right\rangle 2 q\left(e_{n}\right)-\left\langle x, e_{n}\right\rangle\left\langle e_{-n}, e_{n}\right\rangle=0$ und analog $\left\langle y, e_{-n}\right\rangle=0$. Die Zerlegung setzt sich zu $V=\left(K e_{n} \oplus K e_{-n}\right) \perp K E$ auf den gesamten Vektorraum fort; dabei gilt $\left(K e_{n} \oplus K e_{-n}\right) \cap L=R e_{n} \oplus R e_{-n}$ und $K E \cap L=E$. Daher ist $E$ ein ganzes Gitter in dem 2(n-1)-dimensionalen 
hyperbolischen quadratischen Raum $\left(K E,\left.q\right|_{K E}\right)$. Ist $L^{\prime} \supseteq E$ ein ganzes Gitter in $K E$, so ist auch $\left(R e_{n} \oplus R e_{-n}\right) \perp L^{\prime}$ ein ganzes Gitter in $V$. Aus der Maximalität von $L$ in $V$ folgt, dass auch $E$ in $K E$ maximal ist. Induktiv lassen sich die restlichen Elemente einer Basis konstruieren.

Sei umgekehrt eine solche Basis $\left\{e_{i}, e_{-i} \mid i=1, \ldots, n\right\}$ mit $q\left(e_{i}\right)=0$ und $\left\langle e_{i}, e_{j}\right\rangle=\delta_{i,-j}$ für alle $i, j \in I=\{ \pm 1, \ldots, \pm n\}$ gegeben. Dann ist $L=\oplus_{i \in I} R e_{i}$ offensichtlich ein ganzes Gitter in $V$. Sei $L^{\prime} \supseteq L$ ein ebenfalls ganzes Gitter und $x=\sum \lambda_{i} e_{i} \in L^{\prime}$ für gewisse $\lambda_{i} \in K$. Dann gilt $\lambda_{i}=\left\langle x, e_{-i}\right\rangle \in R$ für alle $i \in\{ \pm 1, \ldots, \pm n\}$, also liegt $x$ in $L$.

Satz 4.3.3. Sei $R$ ein Hauptidealring und sei $(C, N)$ die zerfallende Oktavenalgebra über $K=\operatorname{Quot}(R)$. Dann sind alle Maximalordnungen in $(C, N)$ isomorph.

Wir geben vorab eine kurze Übersicht über die Struktur des unten folgenden Beweises an: Sei $D$ eine Maximalordnung in $(C, N)$.

1. In $D$ existiert eine Zerlegung der Eins in ein hyperbolisches Paar $\left(x_{0}, y_{0}\right)$, d.h. es gilt $e=x_{0}+y_{0}$ mit $N\left(x_{0}\right)=N\left(y_{0}\right)=0$ und $\left\langle x_{0}, y_{0}\right\rangle=1$.

2. Es existiert eine orthogonale Zerlegung

$$
D=\left(R x_{0} \oplus R y_{0}\right) \perp(F \oplus G)
$$

mit gewissen total isotropen Unterräumen $F$ und $G$.

3. Es existieren eine Basis $x_{1}, x_{2}, x_{3} \in F$ und eine Basis $y_{1}, y_{2}, y_{3} \in G$, für die $\left\langle x_{i}, y_{j}\right\rangle=\delta_{i j}$ für alle $i, j=1,2,3$ gilt.

4. Die Multiplikation in $D$ ist durch

$$
\begin{aligned}
& x_{0}^{2}=x_{0}, \quad y_{0}^{2}=y_{0}, \quad x_{0} y_{0}=y_{0} x_{0}=0 y, \\
& x_{0} x=x, \quad x_{0} y=0, \quad x x_{0}=0, \quad y x_{0}=y, \\
& y_{0} x=0, \quad y_{0} y=y, \quad x y_{0}=x, \quad y y_{0}=0
\end{aligned}
$$

für alle $x \in F$ und $y \in G$ und durch

$$
\begin{aligned}
& x_{i}^{2}=0, \quad x_{i} x_{i+1}=y_{i+2}, \quad x_{j} x_{i}=-x_{i} x_{j}, \quad x_{i} y_{j}=-\delta_{i j} x_{0}, \\
& y_{i}^{2}=0, \quad y_{i} y_{i+1}=x_{i+2}, \quad y_{j} y_{i}=-y_{i} y_{j}, \quad y_{i} x_{j}=-\delta_{i j} y_{0}
\end{aligned}
$$

für alle $i, j=1,2,3$ gegeben. Die Indizes $i+1, i+2$ sind dabei entsprechend modulo 3 auf 1,2 oder 3 zu reduzieren.

Im Beweis werden wir mehrfach ausnutzen, dass der Assoziator eine alternierende Funktion ist. Insbesondere gelten $-\{a, b, c\}=\{b, a, c\}=\{a, c, b\}$ oder in umgeformter Form

$$
\begin{aligned}
& a(b c)=(a b) c+(b a) c-b(a c), \\
& (a b) c=a(b c)-(a c) b+a(c b)
\end{aligned}
$$


für alle $a, b, c \in C$. Außerdem werden wir verwenden, dass für zwei Elemente $x_{0}, y_{0} \in C$ mit $x_{0}+y_{0}=e$ stets $K\left[x_{0}, y_{0}, z\right]=K\left[x_{0}, z\right]$ assoziativ ist.

BewEIs. Wir folgen der Beweisführung in [vBS59, (3.4)] mit einigen zusätzlichen Ausführungen. - Als zerfallende Oktavenalgebra ist $(C, N)$ insbesondere ein hyperbolischer Raum von Dimension $2 \cdot 4$. Da eine Maximalordnung $D$ nach Lemma 4.2.8 auch als Gitter maximal ist, besitzt sie nach dem vorhergehenden Lemma eine Basis $\left\{e_{i}, e_{-i} \mid i=1,2,3,4\right\}$ über $R$ mit $N\left(e_{i}\right)=0$ und $\left\langle e_{i}, e_{j}\right\rangle=\delta_{i,-j}$ für alle $i, j \in\{ \pm 1, \pm 2, \pm 3, \pm 4\}$. Die Linearkombination der Eins $e=\sum \alpha_{i} e_{i}$ liefert zwei Elemente $x_{0}=\sum_{i>0} \alpha_{i} e_{i}$ und $y_{0}=\sum_{i<0} \alpha_{i} e_{i}$, für die $x_{0}, y_{0} \in D$ und

$$
e=x_{0}+y_{0}, \quad N\left(x_{0}\right)=N\left(y_{0}\right)=0, \quad\left\langle x_{0}, y_{0}\right\rangle=1
$$

gilt, denn für Letzteres gilt $\left\langle x_{0}, y_{0}\right\rangle=\sum_{j<0<i} \alpha_{i} \alpha_{j}\left\langle e_{i}, e_{j}\right\rangle=N(e)=1$. Unter Verwendung von $x_{0}+y_{0}=e$ ergibt sich $\left\langle x_{0}, e\right\rangle=2 N\left(x_{0}\right)+\left\langle x_{0}, y_{0}\right\rangle=1$ und

$$
\begin{aligned}
\overline{x_{0}} & =\left\langle x_{0}, e\right\rangle e-x_{0}=y_{0}, \\
x_{0} y_{0} & =x_{0} \overline{x_{0}}=N\left(x_{0}\right) e=0, \\
x_{0}^{2} & =x_{0}^{2}+x_{0} y_{0}=x_{0} e=x_{0} .
\end{aligned}
$$

Wie im letzten Beweis induzieren $x_{0}$ und $y_{0}$ eine orthogonale Zerlegung $D=$ $\left(R x_{0} \oplus R y_{0}\right) \perp E$ mit $E=\left\{x \in D \mid\left\langle x, x_{0}\right\rangle=0,\left\langle x, y_{0}\right\rangle=0\right\}$. Für $z \in E$ gilt insbesondere $\langle z, e\rangle=0$ und damit $\bar{z}=-z$. Es sind

$$
F:=\left\{x \in E \mid x_{0} x=x\right\}, \quad G:=\left\{y \in E \mid y_{0} y=y\right\},
$$

$F_{0}:=R x_{0} \oplus F$ und $G_{0}:=R y_{0} \oplus G$ vier $R$-Untermoduln von $D$, die total isotrop sind, denn für alle $x, x^{\prime} \in F$ gilt $N(x)=N\left(x_{0} x\right)=0$ und $\left\langle x, x^{\prime}\right\rangle=$ $\left\langle x_{0} x, x_{0} x^{\prime}\right\rangle=N\left(x_{0}\right)\left\langle x, x^{\prime}\right\rangle=0$. Ist $z \in F_{0} \cap G_{0}$, so gilt $z=\left(x_{0}+y_{0}\right) z=2 z$ und somit $z=0$. Für jedes $z \in E$ gilt $\left\langle x_{0} z, x_{0}\right\rangle=N\left(x_{0}\right)\langle z, e\rangle=0$ und $\left\langle x_{0} z, y_{0}\right\rangle=$ $\left\langle z, \overline{x_{0}} y_{0}\right\rangle=\left\langle z, y_{0}\right\rangle=0$ und damit $x_{0} z \in E$. Außerdem ist $x_{0}\left(x_{0} z\right)=x_{0}^{2} z=x_{0} z$, sodass wir insgesamt $x_{0} z \in F$ und analog auch $y_{0} z \in G$ für $z \in E$ erhalten. Aus $z=x_{0} z+y_{0} z$ folgt

$$
E=F \oplus G \quad \text { und } \quad D=F_{0} \oplus G_{0} .
$$

Nebenbei ergibt sich noch $F_{0}=\left\{x \in D \mid x_{0} x=x\right\}, G_{0}=\left\{y \in D \mid y_{0} y=y\right\}$. Sei $x \in F$. Dann gilt $x_{0}\left(x y_{0}\right)=\left(x_{0} x\right) y_{0}=x y_{0}$ und damit $x y_{0} \in F_{0}$. Da $F_{0}$ total isotrop ist, folgt $\left\langle x y_{0}, x^{\prime}\right\rangle=0=\left\langle x, x^{\prime}\right\rangle$ für alle $x^{\prime} \in F_{0}$. Da außerdem

$$
\begin{aligned}
\left\langle x y_{0}, y_{0}\right\rangle & =\langle x, e\rangle N\left(y_{0}\right)=0=\left\langle x, y_{0}\right\rangle \\
\left\langle x y_{0}, y\right\rangle & =\left\langle\overline{y_{0}} \cdot \bar{x}, \bar{y}\right\rangle=\left\langle x_{0}(-x),-y\right\rangle=\langle x, y\rangle
\end{aligned}
$$


für alle $y \in G$ gilt, folgt $\left\langle x y_{0}, z\right\rangle=\langle x, z\rangle$ für alle $z \in D$. Somit gilt $x y_{0}=x$ und damit auch $x x_{0}=0$ für jedes $x \in F$. Damit erhalten wir den Anfang einer Multiplikationstafel in $D$, nämlich

$$
\begin{aligned}
& x_{0}^{2}=x_{0}, \quad y_{0}^{2}=y_{0}, \quad x_{0} y_{0}=y_{0} x_{0}=0 y, \\
& x_{0} x=x, \quad x_{0} y=0, \quad x x_{0}=0, \quad y x_{0}=y, \\
& y_{0} x=0, \quad y_{0} y=y, \quad x y_{0}=x, \quad y y_{0}=0
\end{aligned}
$$

für alle $x \in F$ und $y \in G$.

Es gilt $C=K F_{0} \oplus K G_{0}$, und auch $K F_{0}$ und $K G_{0}$ sind total isotrope Unterräume von $C$. Da $C$ nichtausgeartet ist, folgt $\operatorname{dim}_{K} K F_{0}=4$ und $\operatorname{dim}_{K} K F=3$. Wir konstruieren eine neue Basis von $E=F \oplus G$. Dazu sei in der Konstruktion einer Basis im letzten Lemma $x_{3}=e_{3}$ ein beliebiges primitives Element aus $F$. Ist $e_{-3} \in E$ das zugehörige dort konstruierte Basiselement mit $\left\langle x_{3}, e_{-3}\right\rangle=1$, so gilt für $y_{3}=y_{0} e_{-3} \in G$ ebenfalls $\left\langle x_{3}, y_{3}\right\rangle=\left\langle x_{3}, e_{-3}\right\rangle-\left\langle x_{3}, x_{0} e_{-3}\right\rangle=1$. Induktiv erhalten wir eine Basis $x_{1}, x_{2}, x_{3} \in F$ und $y_{1}, y_{2}, y_{3} \in G$ von $E=F \oplus G$, für die

$$
N\left(x_{i}\right)=N\left(y_{i}\right)=0, \quad\left\langle x_{i}, x_{j}\right\rangle=\left\langle y_{i}, y_{j}\right\rangle=0 \quad \text { und } \quad\left\langle x_{i}, y_{j}\right\rangle=\delta_{i j}
$$

gilt. Es folgt sofort $x_{i}^{2}=x_{i}\left(x_{0} x_{i}\right)=\left(x_{i} x_{0}\right) x_{i}=0$ und analog $y_{i}^{2}=0$ für alle $i=1,2,3$. Aus

$$
\begin{aligned}
\left\langle x_{1} x_{2}, x_{0}\right\rangle & =\left\langle x_{2}, \overline{x_{1}} x_{0}\right\rangle=-\left\langle x_{2}, x_{1} x_{0}\right\rangle=0, \\
\left\langle x_{1} x_{2}, y_{0}\right\rangle & =-\left\langle x_{1}, y_{0} x_{2}\right\rangle=0, \\
y_{0}\left(x_{1} x_{2}\right) & =\left(y_{0} x_{1}\right) x_{2}-x_{1}\left(y_{0} x_{2}\right)+\left(x_{1} y_{0}\right) x_{2}=x_{1} x_{2}
\end{aligned}
$$

folgt $x_{1} x_{2} \in G$, und aus $\left\langle x_{1} x_{2}, x_{1}\right\rangle=N\left(x_{1}\right)\left\langle x_{2}, e\right\rangle=0$ und $\left\langle x_{1} x_{2}, x_{2}\right\rangle=0$ ergibt sich $x_{1} x_{2}=\lambda y_{3}$ mit $\lambda=\left\langle x_{1} x_{2}, x_{3}\right\rangle \in R$. Da $\left\langle x_{2} x_{3}, x_{1}\right\rangle=-\left\langle x_{2}, x_{1} x_{3}\right\rangle=$ $\left\langle x_{1} x_{2}, x_{3}\right\rangle$ gilt, folgt auch $x_{2} x_{3}=\lambda y_{1}$ und entsprechend $x_{3} x_{1}=\lambda y_{2}$ und außerdem $x_{j} x_{i}=-x_{i} x_{j}$.

Zur Berechnung der gemischten Produkte $x_{i} y_{j}$ nutzen wir unter anderem aus, dass $x_{i} x_{j} \in G$ und $y_{i} y_{j} \in F$ gilt (für $i=j$ ist $x_{i}^{2}=0 \in G$ ) und dass $G$ und $F$ total isotrop sind. Es ergibt sich

$$
\begin{aligned}
& \left\langle x_{i} y_{j}, x_{\ell}\right\rangle=-\left\langle y_{j}, x_{i} x_{\ell}\right\rangle=0 \\
& \left\langle x_{i} y_{j}, y_{\ell}\right\rangle=-\left\langle x_{i}, y_{\ell} y_{j}\right\rangle=0 \\
& \left\langle x_{i} y_{j}, x_{0}\right\rangle=-\left\langle x_{i}, x_{0} y_{j}\right\rangle=0 \\
& \left\langle x_{i} y_{j}, y_{0}\right\rangle=-\left\langle x_{i}, y_{0} y_{j}\right\rangle=-\left\langle x_{i}, y_{j}\right\rangle=-\delta_{i j}
\end{aligned}
$$

und damit $x_{i} y_{j}=-\delta_{i j} x_{0}$ und $y_{i} x_{j}=-\delta_{i j} y_{0}$ für alle $i, j, \ell \in\{1,2,3\}$.

Des Weiteren gilt $\lambda y_{3} y_{1}=\left(x_{1} x_{2}\right) y_{1}=x_{1}\left(x_{2} y_{1}\right)-\left(x_{1} y_{1}\right) x_{2}+x_{1}\left(y_{1} x_{2}\right)=$ $0-\left(-x_{0}\right) x_{2}+0=x_{2}$. Da aber auch $y_{3} y_{1}=\mu x_{2}$ mit einem $\mu \in R$ gilt, folgt, dass $\lambda$ eine Einheit in $R$ ist und $\mu=\lambda^{-1}$ gilt. 
Ersetzen wir $x_{3}$ durch $\lambda^{-1} x_{3}$ und $y_{3}$ durch $\lambda y_{3}$, so erhalten wir eine Basis, die alle Eigenschaften von zuvor und zusätzlich noch $x_{1} x_{2}=y_{3}$ und $y_{1} y_{2}=x_{3}$ (usw.) erfüllt. Innerhalb von $E=F \oplus G$ erhalten wir daher die Multiplikationstabelle

$$
\begin{aligned}
& x_{i}^{2}=0, \quad x_{i} x_{i+1}=y_{i+2}, \quad x_{j} x_{i}=-x_{i} x_{j}, \quad x_{i} y_{j}=-\delta_{i j} x_{0}, \\
& y_{i}^{2}=0, \quad y_{i} y_{i+1}=x_{i+2}, \quad y_{j} y_{i}=-y_{i} y_{j}, \quad y_{i} x_{j}=-\delta_{i j} y_{0}
\end{aligned}
$$

für alle $i, j \in\{1,2,3\}$. Die Indizes $i+1, i+2$ sind dabei entsprechend modulo 3 auf 1,2 oder 3 zu reduzieren.

Mit den beiden Multiplikationstafeln (4.2) und (4.4) und der Angabe der Normen und inneren Produkte der Basiselemente in (4.1) und (4.3) haben wir eine vollständige Beschreibung aller Strukturen der Maximalordnung

$$
D=\left(R x_{0} \oplus R y_{0}\right) \perp(F \oplus G)
$$

gefunden. Sind zwei Maximalordnungen in $(C, N)$ mit entsprechenden Basen $\left\{x_{i}, y_{i} \mid i=0,1,2,3\right\}$ und $\left\{x_{i}^{\prime}, y_{i}^{\prime} \mid i=0,1,2,3\right\}$ gegeben, so definiert die Zuordnung $x_{i} \mapsto x_{i}^{\prime}$ und $y_{i} \mapsto y_{i}^{\prime}$ offensichtlich einen bijektiven $R$-Modulhomomorphismus, der zudem die Multiplikation und die Norm respektiert.

\subsection{Maximalordnungen über diskreten Bewertungsringen}

In diesem Abschnitt sei $R$ ein diskreter Bewertungsring mit Quotientenkörper $K$ und Bewertung $v: K^{\times} \rightarrow \mathbb{Z}$.

\subsubsection{Divisionsalgebren über vollständigen Körpern}

Wir betrachten zunächst die nicht zerfallenden Kompositionsalgebren, also die Kompositionsdivisionsalgebren, über vollständigen diskret bewerteten Körpern.

Sei $K$ vollständig bezüglich $v$, und sei $A$ eine Quaternionendivisionsalgebra über $K$. Dann ist die eindeutige Maximalordnung in $A$ der ganze Abschluss $\Delta$ von $R$ in $D$. Dieser stimmt nach Fortsetzung der Bewertung von $K$ auf $A$ mit dem Bewertungsring in $A$ überein.

Im Folgenden werden wir versuchen, diese Charakterisierung auf nicht zerfallende Oktavenalgebren zu übertragen. Zu bemerken ist allerdings, dass über einem vollständigen diskret bewerteten Körper, dessen Restklassenkörper endlich ist, alle Oktavenalgebren zerfallen.

Über einem vollständigen diskreten Bewertungsring kann man die Ganzheit eines Elementes $x$ einer Kompositionsdivisionsalgebra bereits an seiner Norm $N(x)$ erkennen: 
Lemma 4.4.1. Sei $R$ ein vollständiger diskreter Bewertungsring, und sei $(C, N)$ eine Kompositionsalgebra über $K=\operatorname{Quot}(R)$. Ist $C$ eine Divisionsalgebra, so gilt für jedes $x \in C$ mit $N(x) \in R$ auch $\langle x, e\rangle \in R$.

BEwEIS. Für $x=\lambda e$ gilt $N(x)=\lambda^{2}$ und $\langle x, e\rangle=2 \lambda$. Als Bewertungsring ist $R$ ganzabgeschlossen, sodass aus $\lambda^{2} \in R$ bereits $\lambda \in R$ folgt.

Für $x \notin K e$ ist $f=T^{2}-\langle x, e\rangle T+N(x)$ das Minimalpolynom von $x$ und somit irreduzibel. Ist $N(x) \in R$, so folgt aus einem Korollar zum Henselschen Lemma, dass auch der andere Koeffizient, also $-\langle x, e\rangle$, in $R$ liegt, siehe etwa [Neu92, II (4.7) Korollar] oder [Ker07, Satz 12.2].

Satz 4.4.2. Sei $R$ ein vollständiger diskreter Bewertungsring mit Quotientenkörper $K$ und Bewertung $v: K^{\times} \rightarrow \mathbb{Z}$. Sei $(C, N)$ eine Kompositionsdivisionsalgebra über $K$. Dann existiert eine eindeutige Exponentialbewertung $w$ auf $C^{\times}$, die $v$ fortsetzt, nämlich

$$
w: C^{\times} \rightarrow \frac{1}{2} \mathbb{Z}, x \mapsto \frac{1}{2} v(N(x)) .
$$

Außerdem ist $C$ vollständig bezüglich $w$.

Dabei sei in Analogie zum assoziativen Fall eine Exponentialbewertung auf $C^{\times}$eine Abbildung $w: C^{\times} \rightarrow \mathbb{R}$, die $w(x y)=w(x)+w(y)$ und $w(x+y) \geq$ $\min (w(x), w(y))$ für alle $x, y \in C^{\times}$erfüllt. Auch hier wird zusätzlich $w(0)=\infty$ definiert.

Ist $\operatorname{dim} C \leq 4$, so ist $C$ ein (assoziativer) Körper oder Schiefkörper über $K$ und $w$ ist die bekannte eindeutige Exponentialbewertung auf $C$, die $v$ fortsetzt.

BEwEIs. Wir verfahren großteils analog zum assoziativen Fall, siehe etwa [Ker07, Satz 12.4] für Schiefkörper.

Sei zunächst $w: x \mapsto \frac{1}{2} v(N(x))$ die angegebene Abbildung. Für $\lambda \in K$ gilt dann $w(\lambda e)=\frac{1}{2} v(N(\lambda e))=\frac{1}{2} v\left(\lambda^{2}\right)=v(\lambda)$, also ist $w$ eine Fortsetzung von $v$. Aus der Multiplikativität der Norm und der entsprechenden Eigenschaft für $v$ folgt $w(x y)=w(x)+w(y)$ für alle $x, y \in C^{\times}$.

Für jedes $z \in C^{\times}$mit $w(z) \geq 0$ gilt $N(z) \in R$ und nach dem obigen Lemma ist dann auch $\langle z, e\rangle \in R$. Damit ist $N(z+e)=N(z)+1+\langle z, e\rangle \in R$ und somit gilt $w(z+e)=\frac{1}{2} v(N(z+e)) \geq 0$. Für $x, y \in C^{\times}$mit $w(x) \geq w(y)$ gilt $w\left(x y^{-1}\right) \geq 0$ und somit auch $w\left(x y^{-1}+e\right) \geq 0$. Es folgt

$$
w(x+y)=w\left(x y^{-1}+e\right)+w(y) \geq w(y)=\min (w(x), w(y)) .
$$

Sei $w^{\prime}: C^{\times} \rightarrow \mathbb{R}$ eine zweite Exponentialbewertung, die $v$ fortsetzt. Es gilt für $\lambda \in K^{\times}$somit $w^{\prime}(\lambda e)=v(\lambda)=w(\lambda e)$. Sei $z \in C^{\times}$mit $w(z)=0$. Dann gilt wiederum $v(\langle z, e\rangle) \geq 0$ und für $w^{\prime}$ folgt

$$
2 w^{\prime}(z)=w^{\prime}\left(z^{2}\right)=w^{\prime}(\langle z, e\rangle z-N(z) e) \geq \min \left(v(\langle z, e\rangle)+w^{\prime}(z), v(N(z))\right)
$$


und daher $w^{\prime}(z) \geq v(\langle z, e\rangle) \geq 0$ oder $w^{\prime}(z) \geq \frac{1}{2} v(N(z))=0$. Außerdem ist $w\left(z^{-1}\right)=-w(z)=0$, sodass sich auch $-w^{\prime}(z)=w^{\prime}\left(z^{-1}\right) \geq 0$ und damit insgesamt $w^{\prime}(z)=0$ ergibt. Sei $\pi \in R$ eine Uniformisierende mit $v(\pi)=1$. Für beliebiges $x \in C^{\times}$gilt dann $w\left(\pi^{-2 w(x)} x^{2}\right)=0$ und somit

$$
2 w^{\prime}(x)=v\left(\pi^{2 w(x)}\right)+w^{\prime}\left(\pi^{-2 w(x)} x^{2}\right)=v\left(\pi^{2 w(x)}\right)+w\left(\pi^{-2 w(x)} x^{2}\right)=2 w(x) .
$$

Die Vollständigkeit von $C$ bezüglich $w$ folgt aus Lemma 4.4.3.

Lemma 4.4.3. Sei L ein Körper, der vollständig bezüglich einer Betragsbewertung $|\cdot|: L \rightarrow \mathbb{R}_{\geq 0}$ ist, und sei $\|\cdot\|: V \rightarrow \mathbb{R}_{\geq 0}$ eine Norm auf einem endlichdimensionalen $L$-Vektorraum $V$ mit $\|\lambda x\|=|\lambda| \cdot\|x\|$ für alle $\lambda \in L, x \in V$. Dann ist $V$ ebenfalls vollständig.

Beweis. Siehe etwa [Ker07, Abschnitt 12.5]: Auch wenn der Beweis nur für Schiefkörper $D$ formuliert ist, nutzt er die Multiplikation auf $D$ nicht aus und ist allgemeiner für Vektorräume gültig.

Für assoziative Divisionsalgebren $A$ (beliebiger Dimension $n$ ) erfolgt die Konstruktion der Fortsetzung $w$ meist als $w(x)=\frac{1}{n} v\left(N_{A / K}(x)\right)$ für $x \in A^{\times}$mit Hilfe der Algebrennorm $N_{A / K}: A \rightarrow K, x \mapsto \operatorname{det}\left(\ell_{x}\right)$, wobei $\ell_{x}: A \rightarrow A, z \mapsto x z$ die Linksmultiplikation mit $x$ bezeichne.

Übernehmen wir diese Konstruktion auch für nichtassoziative Algebren, so kann man analog zum assoziativen Fall (siehe etwa [Ker07, Lemma 12.3]) für eine Oktavendivisionsalgebra $(C, N)$ nachrechnen, $\operatorname{dass} \operatorname{det}\left(\ell_{x}\right)=N(x)^{4}$ und damit auch hier $w(x)=\frac{1}{8} v\left(\operatorname{det}\left(\ell_{x}\right)\right)$ für alle $x \in C^{\times}$gilt. Aus der Multiplikativität der Kompositionsalgebrennorm $N$ folgt außerdem, dass auch die Zuordnung $x \mapsto \operatorname{det}\left(\ell_{x}\right)$ auf $C$ multiplikativ ist. Dies wäre sonst nicht unmittelbar offensichtlich, da im Allgemeinen $\ell_{x} \ell_{y}=\ell_{x y}+\ell_{y x}-\ell_{y} \ell_{x} \neq \ell_{x y}$ ist.

Sei $(C, N)$ nun wieder eine Kompositionsdivisionsalgebra beliebiger Dimension. Für die Fortsetzung $w: C^{\times} \rightarrow \frac{1}{2} \mathbb{Z}$ der Bewertung gilt

$$
w(e)=0, \quad w(-x)=w(x), \quad w\left(x^{-1}\right)=-w(x), \quad w(\bar{x})=w(x)
$$

für alle $x \in C^{\times}$. Die letzte Formel rechnet man dabei über das Inverse nach: Es ist $w(\bar{x})=w\left(N(x) x^{-1}\right)=v(N(x))+w\left(x^{-1}\right)=2 w(x)-w(x)=w(x)$. Außerdem gilt für die verschärfte Dreiecksungleichung auch hier

$$
w(x+y)=\min (w(x), w(y)) \text { für } w(x) \neq w(y),
$$

denn es ist $w(y)=w((x+y)-x) \geq \min (w(x+y), w(x))$; ist nun $w(x)>w(y)$, so muss $w(y) \geq w(x+y)$ sein, zusammen mit $w(x+y) \geq \min (w(x), w(y))=w(y)$ ergibt sich die Behauptung. 
Lemma 4.4.4. Sei $(C, N)$ eine Kompositionsdivisionsalgebra über dem vollständigen diskret bewerteten Körper $K=\operatorname{Quot}(R)$, und sei $w: C^{\times} \rightarrow \frac{1}{2} \mathbb{Z}$ die eindeutige Fortsetzung der Bewertung von K. Dann ist

$$
\Delta=\{x \in C \mid w(x) \geq 0\}
$$

eine $R$-Unteralgebra von $C$, die genau die ganzen Elemente von $C$ über $R$ enthält. Es gilt $K \Delta=C$. Außerdem besitzt $\Delta$ genau ein maximales Ideal $\mathfrak{m}_{w}$, und für dieses und die Menge der Einheiten gilt:

$$
\begin{aligned}
\mathfrak{m}_{w} & =\{x \in C \mid w(x)>0\}, \\
\Delta^{\times} & =\{x \in C \mid w(x)=0\} .
\end{aligned}
$$

Beweis. Da $w(x)=\frac{1}{2} v(N(x))$ gilt und $R$ der Bewertungsring von $v: K^{\times} \rightarrow \mathbb{Z}$ ist, enthält $\Delta$ genau die Elemente $x$ mit $N(x) \in R$. Nach Lemma 4.4.1 sind das genau die ganzen Elemente von $C$.

Sei $\pi \in K$ eine Uniformisierende mit $v(\pi)=1$. Ist $w(x) \geq 0$, so gilt $x \in \Delta$. Ist $w(x)<0$, so sei $z=\pi^{-2 w(x)} x$. Dann gilt $w(z)=-w(x)>0$ und somit ist $x=\pi^{2 w(x)} \cdot z \in K \Delta$.

Die anderen Aussagen folgen direkt aus den definierenden Eigenschaften einer Exponentialbewertung.

Sei $\mathfrak{m}$ das maximale Ideal im Bewertungsring $R$ und $k=R / \mathfrak{m}$ der Restklassenkörper. Dann ist $\Delta / \mathfrak{m}_{w}$ eine nicht notwendig assoziative $k$-Algebra; die Verknüpfungen der Restklassen seien dabei durch die ihrer Vertreter in $\Delta$ und $R$ induziert. Dass Addition und Skalarmultiplikation wohldefiniert sind, folgt daraus, dass $\mathfrak{m}_{w}$ ein $R$-Untermodul des $R$-Moduls $\Delta$ ist und dass $\mathfrak{m} \Delta \subseteq \mathfrak{m}_{w}$ gilt; die Wohldefiniertheit der Multiplikation folgt daraus, dass $\mathfrak{m}_{w}$ ein zweiseitiges Ideal ist.

Wie im assoziativen Fall heiße $f=\operatorname{dim}_{k} \Delta / \mathfrak{m}_{w}$ der Trägheitsgrad von $C$ über $K$ bzw. von $w$ über $v$.

Lemma 4.4.5. Sei $(C, N)$ eine Kompositionsdivisionsalgebra über dem vollständigen diskret bewerteten Körper $K=\operatorname{Quot}(R)$. Dann gilt $f \leq \operatorname{dim}_{K} C$ für den Trägheitsgrad $f$ von $C$.

BeweIs. Sei $n=\operatorname{dim}_{K} C$. Dann sind je $n+1$ Elemente $x_{1}, \ldots, x_{n+1} \in \Delta$ über $K$ linear abhängig und es gibt eine nichttriviale Linearkombination $\sum \lambda_{i} x_{i}=0$ mit $\lambda_{i} \in K$. Wähle ein $j \in\{1, \ldots, n+1\}$ so, dass $v\left(\lambda_{j}\right)$ minimal ist. Dann gilt $\lambda_{j}^{-1} \lambda_{j}=1$ und $\left(\lambda_{j}^{-1} \lambda_{i}\right) \in R$, sodass $\sum\left(\left(\lambda_{j}^{-1} \lambda_{i}\right)+\mathfrak{m}\right)\left(x_{i}+\mathfrak{m}_{w}\right)$ eine nichttriviale Darstellung der 0 in $\Delta / \mathfrak{m}_{w}$ ist. Folglich sind je $n+1$ Elemente in $\Delta / \mathfrak{m}_{w}$ linear abhängig über $k$ und es gilt $f=\operatorname{dim}_{k} \Delta / \mathfrak{m}_{w} \leq n$.

Für die eindeutige Fortsetzung $w: C^{\times} \rightarrow \frac{1}{2} \mathbb{Z}$ der Bewertung von $K$ gilt $w\left(C^{\times}\right)=\frac{1}{e} \mathbb{Z}$ mit $e=1$ oder $e=2$. Mit Hilfe dieses Verzweigungsindexes e können wir $w$ zu einer diskreten Bewertung $v^{\prime}: C^{\times} \rightarrow \mathbb{Z}, x \mapsto e \cdot w(x)$ 
mit $v^{\prime}\left(C^{\times}\right)=\mathbb{Z}$ normieren. Für diese gilt dann $v^{\prime}\left(\lambda e_{C}\right)=e \cdot v(\lambda)$ für $\lambda \in K^{\times}$ (dabei bezeichne $e_{C}$ das Einselement in $C$ ). Ein jedes Element $\pi_{w} \in C$ mit $w\left(\pi_{w}\right)=\frac{1}{e}$ bzw. $v^{\prime}\left(\pi_{w}\right)=1$ heißt dann Uniformisierende oder Primelement bezüglich $w$. Sei $\pi_{w} \in C$ eine solche Uniformisierende. Dann existieren zu jedem $x \in K^{\times}$eine Einheit $u \in \Delta^{\times}$und ein $n \in \mathbb{Z}$ mit $x=u \pi_{w}^{n}$, nämlich $u=x \pi_{w}^{-n}$ und $n=e w(x)=v^{\prime}(x)$.

Satz 4.4.6. Sei $(C, N)$ eine Kompositionsdivisionsalgebra über dem vollständigen diskret bewerteten Körper $K=\operatorname{Quot}(R)$. Dann ist $\Delta$ die eindeutige Maximalordnung in $(C, N)$.

Beweis. Ist $e=1$, so gilt $\mathfrak{m}_{w}=\mathfrak{m} \Delta$ und $\Delta / \mathfrak{m}_{w}=\Delta / \mathfrak{m} \Delta=k \otimes \Delta$. Insbesondere ist $\Delta / \mathfrak{m} \Delta$ endlich erzeugt als $R / \mathfrak{m}$-Modul. Da $(R, \mathfrak{m})$ ein (kommutativer) lokaler Ring ist, folgt aus Nakayamas Lemma, dass auch $\Delta$ über $R$ endlich erzeugt ist. Damit ist $\Delta$ eine Ordnung in $C$, die zudem alle ganzen Elemente enthält.

Ist $e=2$, so sei $\pi_{w} \in C$ eine Uniformisierende mit $w\left(\pi_{w}\right)=\frac{1}{2}$. Dann ist $K\left[\pi_{w}\right]=K e_{C} \oplus K \pi_{w} \subseteq C$ eine Körpererweiterung von $K$ von Grad 2 mit diskreter Bewertung $v^{\prime}:=2 w$. Für $\lambda, \mu \in K$ gilt $w\left(\lambda e_{C}\right)=v(\lambda) \in \mathbb{Z}$ und $w\left(\mu \pi_{w}\right)=v(\mu)+\frac{1}{2} \notin \mathbb{Z}$ und somit $w\left(\lambda e_{C}+\mu \pi_{w}\right)=\min \left(v(\lambda), v(\mu)+\frac{1}{2}\right)$. Für $x=\lambda e_{C}+\mu \pi_{w}$ gilt daher genau dann $v^{\prime}(x)=2 w(x) \geq 0$, wenn $v(\lambda) \geq 0$ und $v(\mu) \geq 0$ sind. Also ist $R\left[\pi_{w}\right]=R e_{C} \oplus R \pi_{w}$ der Bewertungsring von $K\left[\pi_{w}\right]$ bezüglich $v^{\prime}$. Sein maximales Ideal ist $\mathfrak{m}^{\prime}=R\left[\pi_{w}\right] \pi_{w}=\mathfrak{m} e_{C}+R \pi_{w}$. Nach Lemma 3.4.4 ist $C$ ein $K\left[\pi_{w}\right]$-Linksvektorraum; ebenso ist $\Delta$ offensichtlich ein $R\left[\pi_{w}\right]$-Linksmodul. Da $\mathfrak{m}^{\prime} \subseteq \mathfrak{m}_{w}$ gilt, ist $\Delta / \mathfrak{m}_{w}$ ein $R\left[\pi_{w}\right] / \mathfrak{m}^{\prime}$-Linksvektorraum von Dimension $\operatorname{dim}_{R\left[\pi_{w}\right] / \mathfrak{m}^{\prime}} \Delta / \mathfrak{m}_{w} \leq f \leq \operatorname{dim}_{K} C$. Da für die maximalen Ideale sogar $\mathfrak{m}^{\prime} \Delta=\mathfrak{m}_{w}$ gilt, folgt aus Nakayamas Lemma, dass $\Delta$ ein endlich erzeugter $R\left[\pi_{w}\right]$-Linksmodul ist. Offensichtlich ist $R\left[\pi_{w}\right]$ ein freier $R$-Modul von Rang 2, sodass insgesamt $\Delta$ auch als $R$-Modul endlich erzeugt ist.

Ist $e=1$, so ergibt Nakayamas Lemma, dass $f$ Erzeugende für $\Delta$ über $R$ ausreichen. Andererseits enthält $\Delta$ eine Basis von $C$ über $K$, also mindestens $n=\operatorname{dim}_{K} C$ linear unabhängige Elemente, sodass $n=e f$ folgt. Auch für $e=2$ gilt $n=e f$; der Beweis für assoziative Algebren (siehe etwa [Ker07, Abschnitte 12.11 und 12.12]) lässt sich direkt auf Kompositionsalgebren übertragen.

Korollar 4.4.7. Sei $(C, N)$ eine Kompositionsdivisionsalgebra über dem vollständigen diskret bewerteten Körper $K=\operatorname{Quot}(R)$. Dann ist $(\Delta, N)$ eine Kompositionsalgebra über $R$.

BEweis. Lemma 4.3.1 besagt, dass jede Ordnung über einem Hauptidealring eine Kompositionsalgebra ist.

Da $w(\bar{x})=w(x)$ für alle $x \in C$ gilt, sind $\Delta$ und $\mathfrak{m}_{w}$ abgeschlossen unter Konjugation, und diese induziert eine wohldefinierte Standard-Involution auf $\Delta / \mathfrak{m}_{w}$ über $k$, nämlich $\Delta / \mathfrak{m}_{w} \rightarrow \Delta / \mathfrak{m}_{w}, x+\mathfrak{m}_{w} \mapsto \bar{x}+\mathfrak{m}_{w}$. Daher induzieren auch die 
Norm $N$ und ihre zugehörige Bilinearform eine wohldefinierte multiplikative quadratische Form $N: \Delta / \mathfrak{m}_{w} \rightarrow k, x+\mathfrak{m}_{w} \mapsto N(x)+\mathfrak{m}$ mit Bilinearform $\left\langle x+\mathfrak{m}_{w}, y+\mathfrak{m}_{w}\right\rangle=\langle x, y\rangle+\mathfrak{m}$ auf der Restklassenalgebra $\Delta / \mathfrak{m}_{w}$.

Satz 4.4.8. Sei $R$ ein vollständiger diskreter Bewertungsring, und sei $(C, N)$ eine Kompositionsdivisionsalgebra über $K=\operatorname{Quot}(R)$. Ist $2 \in R^{\times}$eine Einheit, so ist $\left(\Delta / \mathfrak{m}_{w}, N\right)$ eine Kompositionsalgebra über dem Restklassenkörper $k=$ $R / \mathfrak{m}$.

BEwEIS. Nach den vorherigen Überlegungen ist nur noch zu zeigen, dass $N$ auf $\Delta / \mathfrak{m}_{w}$ nichtausgeartet ist. Es ist 2 genau dann in $R$ invertierbar, wenn $v(2)=0$ gilt. Sei $x \in \Delta$ so, dass $\left\langle x+\mathfrak{m}_{w}, y+\mathfrak{m}_{w}\right\rangle=0 \in R / \mathfrak{m}$ für alle $y \in \Delta$ gilt. Letzteres ist äquivalent zu $\langle x, y\rangle \in \mathfrak{m}$ und damit zu $v(\langle x, y\rangle)>0$ für alle $y \in \Delta$. Insbesondere ist dann auch $0<v(\langle x, x\rangle)=v(2 N(x))=v(2)+v(N(x))=$ $0+2 w(x)$, also ist $x \in \mathfrak{m}_{w}$ und damit $x+\mathfrak{m}_{w}=0$.

\subsubsection{Zerfallende Algebren (über vollständigen Körpern)}

Sei $K$ vollständig bezüglich $v$, und sei $A=\mathbb{M}_{2}(K)$ die zerfallende Quaternionenalgebra. Dann sind die Maximalordnungen in $A$ genau die $R$-Unteralgebren $u \mathbb{M}_{2}(R) u^{-1}$ für $u \in A^{\times}=\mathrm{GL}_{2}(K)$. Offensichtlich sind je zwei dieser Maximalordnungen isomorph. Es gilt in kanonischer Weise $u \mathbb{M}_{2}(R) u^{-1}=\operatorname{End}_{R}\left(u R^{2}\right)$, und die unterschiedlichen Maximalordnungen entsprechen den Einbettungen des Gitters $R^{2}$ in $K^{2}$ mit $(0,0) \mapsto(0,0)$.

Auch in Oktavenalgebren sind die Maximalordnungen im Allgemeinen nicht eindeutig bestimmt, aber auch in Dimension 8 gilt Folgendes:

Satz 4.4.9. Sei $R$ ein diskreter Bewertungsring und sei $(C, N)$ die zerfallende Oktavenalgebra über $K=\operatorname{Quot}(R)$. Dann sind alle Maximalordnungen in $(C, N)$ isomorph.

BEweIs. Dies gilt bereits allgemeiner für zerfallende Oktavenalgebren über beliebigen Hauptidealringen, siehe Satz 4.3.3 (oder [vBS59, (3.4)]).

\subsubsection{Algebren über beliebigen diskret bewerteten Körpern}

Sei $R$ nun wieder ein beliebiger diskreter Bewertungsring mit Quotientenkörper $K$, und seien $\hat{R}$ bzw. $\hat{K}$ ihre Vervollständigungen. Dann ist $\hat{R}$ ein treuflacher $R$-Modul und es gilt $\hat{R} \cap K=R$.

Ist $M$ ein endlich erzeugter $R$-Modul, so ist die Abbildung $M \rightarrow \hat{M}=\hat{R} \otimes_{R} M$ injektiv. Sei $V$ ein endlich-dimensionaler $K$-Vektorraum, dann definiert die Zuordnung $L \mapsto \hat{L}$ eine inklusionserhaltende Bijektion mit Umkehrabbildung $L^{\prime} \mapsto L^{\prime} \cap V$ zwischen den $R$-Gittern in $V$ und den $\hat{R}$-Gittern in seiner Vervollständigung $\hat{V}=\hat{K} \otimes_{K} V$. 
Ist $(C, N)$ eine Kompositionsalgebra über $K$, so ist $\left(\hat{C}=\hat{K} \otimes C, \hat{N}=N_{\hat{K}}\right)$ eine Kompositionsalgebra über $\hat{K}$. Die obige Bijektion der Gitter überführt auch Ordnungen bzw. Maximalordnungen ineinander:

Lemma 4.4.10. Sei $(C, N)$ eine Kompositionsalgebra über einem diskret bewerteten Körper $K=\operatorname{Quot}(R)$, und seien $(\hat{C}, \hat{N}), \hat{K}$ und $\hat{R}$ ihre Vervollständigungen. Dann existiert eine inklusionserhaltende Bijektion zwischen den $R$-Ordnungen in $C$ und den $\hat{R}$-Ordnungen in $\hat{C}$. Insbesondere entsprechen die Maximalordnungen in $C$ und in $\hat{C}$ einander.

Beweis. Sei $L$ ein $R$-Gitter in $C$. Da $\hat{L}=\hat{R} \otimes L$ und $\hat{L} \cap C=L$ gilt, ist offensichtlich, dass $L$ genau dann multiplikativ abgeschlossen ist, wenn $\hat{L}$ es ist, und dass $e \in L$ genau dann gilt, wenn $e \in \hat{L}$ ist.

Sind alle Elemente aus $L$ ganz, so gilt $\hat{N}(\hat{L}) \subseteq \hat{R} \otimes N(L) \subseteq \hat{R}$ und $\langle\hat{L}, e\rangle \subseteq$ $\hat{R}\langle L, e\rangle \subseteq \hat{R}$. Folglich sind auch alle Elemente aus $\hat{L}$ ganz. Sind umgekehrt alle Elemente aus $\hat{L}$ ganz, so gilt $N(x),\langle x, e\rangle \in \hat{R} \cap K=R$ für jedes $x \in L$.

Korollar 4.4.11. Sei $(C, N)$ eine Kompositionsdivisionsalgebra über einem diskret bewerteten Körper $K=\operatorname{Quot}(R)$, für die auch $(\hat{C}, \hat{N})$ eine Kompositionsdivisionsalgebra über $\hat{K}$ ist, und sei $w: \hat{C}^{\times} \rightarrow \mathbb{Z}$ die eindeutige Fortsetzung der Bewertung von $K$ bzw. $\hat{K}$. Dann ist $\Delta_{K}=\{x \in C \mid w(x) \geq 0\}$ die einzige Maximalordnung in $(C, N)$.

\subsection{Maximalordnungen über $\mathbb{Z}$ und über Krullringen}

Über den ganzen Zahlen $\mathbb{Z}$ gibt es - wie etwa für quadratische Formen - auch für Maximalordnungen in nichtausgearteten quadratischen Räumen ein LokalGlobal-Prinzip. Es lässt sich vollständig lokal überprüfen, wann ein Gitter eine Maximalordnung ist:

$\mathrm{Zu}$ einer Primzahl $p \in \mathbb{Z}$ bezeichne $\mathbb{Z}_{p}$ den Ring der ganzen $p$-adischen Zahlen und $\mathbb{Q}_{p}$ den Körper aller $p$-adischen Zahlen.

Satz 4.5.1 ([vBS59, (4.1)]). Sei $(C, N)$ eine Oktavenalgebra über den rationalen Zahlen $\mathbb{Q}$. Dann ist ein Gitter $B$ in $(C, N)$ genau dann eine Maximalordnung, wenn für jede Primzahl $p \in \mathbb{Z}$ das Gitter $B_{p}=B \otimes \mathbb{Z}_{p}$ eine Maximalordnung in der Kompositionsalgebra $C_{p}=C \otimes \mathbb{Q}_{p}$ über $\mathbb{Z}_{p}$ ist.

Dieses Lokal-Global-Prinzip gilt auch allgemeiner über beliebigen Zahlkörpern, über ganzabgeschlossenen noetherschen Ringen und mit gewissen Einschränkungen noch allgemeiner über beliebigen Krullringen, wie wir im folgenden Abschnitt zeigen wollen. 


\subsubsection{Lokalisieren von Maximalordnungen}

Zuerst untersuchen wir das Verhalten von Maximalordnungen oder allgemeiner von Gittern eines nichtausgearteten quadratischen Raumes beim Lokalisieren.

Sei in diesem Abschnitt $R$ ein Integritätsbereich, $K=\operatorname{Quot}(K)$ sein Quotientenkörper und $(V, q)$ ein endlich-dimensionaler Vektorraum mit nichtausgearteter quadratischer Form über $K$.

Lemma 4.5.2. Sei $S \subset R$ eine multiplikative Menge. Dann ist für jedes $R$ Gitter $L$ in $(V, q)$ auch die Lokalisierung $S^{-1} L=S^{-1} R \otimes_{R} L$ ein $S^{-1} R$-Gitter in $(V, q)$ und es gelten

$$
S^{-1}\left(L^{\#}\right) \subseteq\left(S^{-1} L\right)^{\#} \quad \text { und } \quad S^{-1}\left(L^{(\#)}\right) \subseteq\left(S^{-1} L\right)^{(\#)} .
$$

Ist L endlich erzeugt, so gilt jeweils Gleichheit:

$$
S^{-1}\left(L^{\#}\right)=\left(S^{-1} L\right)^{\#} \quad \text { und } \quad S^{-1}\left(L^{(\#)}\right)=\left(S^{-1} L\right)^{(\#)} \text {. }
$$

BeweIs. Als Gitter ist der $R$-Modul $L$ in einem endlich erzeugten $R$-Modul $M$ enthalten. Offensichtlich sind $S^{-1} L$ und $S^{-1} M$ dann $S^{-1} R$-Moduln und $S^{-1} M$ ist endlich erzeugt. Außerdem gilt mit $K L=V$ auch $K\left(S^{-1} L\right)=V$. Daher ist $S^{-1} L$ ein $S^{-1} R$-Gitter in $V$.

Sei $x \in L^{\#}, s \in S$. Für alle $y \in L, t \in S$ gilt dann $\left\langle s^{-1} x, t^{-1} y\right\rangle=(t s)^{-1}\langle x, y\rangle \in$ $S^{-1} R$ und somit $s^{-1} x \in\left(S^{-1} L\right)^{\#}$.

Sei $x \in L^{(\#)}, s \in S$. Dann gilt $q\left(s^{-1} x\right)=s^{-2} q(x) \in S^{-1} R$ und also $s^{-1} x \in$ $\left(S^{-1} L\right)^{(\#)}$

Sei nun $L$ endlich erzeugt. Sei $z \in\left(S^{-1} L\right)^{\#}$. Dann gilt insbesondere für die endlich vielen Erzeugenden $x_{1}, \ldots, x_{m}$ von $L$ über $R$, dass $\left\langle z, x_{i}\right\rangle \in S^{-1} R$ ist. Sei $t$ das Produkt der Nenner dieser Werte, setze $z^{\prime}=t z$. Dann gilt $\left\langle z^{\prime}, x_{i}\right\rangle=t\left\langle z, x_{i}\right\rangle \in R$, also $z^{\prime} \in L^{\#}$ und damit $z=t^{-1} z^{\prime} \in S^{-1}\left(L^{\#}\right)$.

Sei $z \in\left(S^{-1} L\right)^{(\#)}$. Nach dem Vorhergehenden existieren gewisse $z^{\prime} \in L^{\#}, t \in S$ mit $z=t^{-1} z^{\prime}$. Außerdem gilt $q\left(z^{\prime}\right)=t^{2} q(z) \in S^{-1} R$. Ist $s \in S$ dessen Nenner, so setze $z^{\prime \prime}=s z^{\prime}$. Dann gilt auch $z^{\prime \prime} \in L^{\#}$ und $q\left(z^{\prime \prime}\right)=s^{2} q\left(z^{\prime}\right) \in R$, also $z^{\prime \prime} \in L^{(\#)}$ und somit $z=(s t)^{-1} z^{\prime \prime} \in S^{-1}\left(L^{(\#)}\right)$.

Die Aussage für das duale Gitter erhält man im endlich erzeugten Fall auch über die kanonische Isomorphie $L^{\#}=\operatorname{Hom}_{R}(L, R)$ und die Lokalisierung von $\operatorname{Hom}_{R}$ zu $\operatorname{Hom}_{S^{-1} R}$.

Dieses Lemma rechtfertigt für endlich erzeugte Gitter die Notationen

$$
S^{-1} L^{\#} \quad \text { und } \quad S^{-1} L^{(\#)}
$$

für $S^{-1}\left(L^{\#}\right)=\left(S^{-1} L\right)^{\#}$ beziehungsweise für $S^{-1}\left(L^{(\#)}\right)=\left(S^{-1} L\right)^{(\#)}$. Für Primideale $\mathfrak{p} \subset R$ können wir entsprechend $L_{\mathfrak{p}}^{\#}$ und $L_{\mathfrak{p}}^{(\#)}$ schreiben.

Lemma 4.5.3. Sei $L$ ein ganzes $R$-Gitter in $(V, q)$. Ist für jedes maximale Ideal $\mathfrak{m} \subset R$ das lokalisierte Gitter $L_{\mathfrak{m}}$ maximal, so ist auch $L$ maximal. 
BewEIS. Seien alle $R_{\mathfrak{m}}$-Gitter $L_{\mathfrak{m}}$ maximal. Ist $M \supseteq L$ ein ebenfalls ganzes $R$-Gitter, so ist für jedes maximale Ideal $\mathfrak{m} \subset R$ auch $M_{\mathfrak{m}}$ ein ganzes $R_{\mathfrak{m}}$-Gitter mit $M_{\mathfrak{m}} \supseteq L_{\mathfrak{m}}$. Aus der Maximalität von $L_{\mathfrak{m}}$ folgt $M_{\mathfrak{m}}=L_{\mathfrak{m}}$ für jedes $\mathfrak{m}$ und damit $M=L$ (nach Lemma A.3.3).

Wenden wir die Aussage statt auf $R$ auf eine Lokalisierung $R_{\mathfrak{p}}$ in einem beliebigen Primideal an, so erhalten wir: Ist $L_{\mathfrak{m}}$ für jedes maximale Ideal $\mathfrak{m}$ von $R$ ein maximales Gitter, so ist auch $L_{\mathfrak{p}}$ für jedes Primideal $\mathfrak{p}$ von $R$ maximal.

Ist ein maximales Gitter endlich erzeugt, so können wir auch die Umkehrung beweisen:

Lemma 4.5.4. Sei $L$ ein endlich erzeugtes, maximales $R$-Gitter in $(V, q)$, dann ist auch $S^{-1} L$ ein maximales $S^{-1} R$-Gitter für jede multiplikative Menge $S \subset R$. Insbesondere ist dann für jedes Primideal $\mathfrak{p} \subset R$ auch $L_{\mathfrak{p}}$ ein maximales Gitter über $R_{\mathfrak{p}}$.

Beweis. Ist $L$ ein ganzes $R$-Gitter, so ist offensichtlich auch $S^{-1} L$ ein ganzes $S^{-1} R$-Gitter. Ist $L$ maximal, so gilt $L=L^{(\#)}$ und damit $S^{-1} L=S^{-1}\left(L^{(\#)}\right)$. Da $L$ endlich erzeugt ist, folgt aus dem obigen Lemma weiter $S^{-1} L=S^{-1} L^{(\#)}$. Damit ist $S^{-1} L$ ein maximales $S^{-1} R$-Gitter.

Zusammen ergeben die beiden Lemmata ein hinreichendes und notwendiges Kriterium, wann ein endlich erzeugtes Gitter maximal ist - es sei aber nicht gesagt, dass jedes maximale Gitter endlich erzeugt sein muss:

Korollar 4.5.5. Sei L ein endlich erzeugtes, ganzes Gitter in $(V, q)$. Dann ist $L$ genau dann maximal, wenn für jedes maximale Ideal $\mathfrak{m} \subset R$ das lokalisierte Gitter $L_{\mathfrak{m}}$ ein maximales $R_{\mathfrak{m}}$-Gitter ist.

Über noetherschen Ringen ist jedes Gitter endlich erzeugt. Wir können daher stets in den maximalen Idealen testen, ob ein ganzes Gitter maximal ist:

Korollar 4.5.6. Sei $R$ ein noetherscher Ring. Dann ist ein ganzes $R$-Gitter $L$ in $(V, q)$ genau dann maximal, wenn für jedes maximale Ideal $\mathfrak{m} \subset R$ das lokalisierte Gitter $L_{\mathfrak{m}}$ ein maximales $R_{\mathfrak{m}}$-Gitter ist.

\subsubsection{Ganze Gitter über Krullringen}

Zur Definition von Krullringen sei hier aus Bourbakis Kommutativer Algebra die äquivalente Beschreibung aus Theorem 4 ([Bou72, VII, § 1.6]) angegeben. Bourbakis eigentliche Definition ([Bou72, VII, §1.3]) gibt eine allgemeinere Charakterisierung über von den Primidealen unabhängige diskrete Bewertungen; diese liefert natürlich dieselben Krullringe. Eine kurze Zusammenfassung findet sich im Anhang, Abschnitt A.9. 
Definition. Sei $R$ ein Integritätsbereich und $P$ die Menge seiner Primideale von Höhe 1. Dann ist $R$ genau dann ein Krullring, wenn gilt:

1. Für jedes $\mathfrak{p} \in P$ ist $R_{\mathfrak{p}}$ ein diskreter Bewertungsring.

2. Es ist $R$ der Durchschnitt dieser Bewertungsringe: $R=\bigcap_{\mathfrak{p} \in P} R_{\mathfrak{p}}$.

3. Jedes $x \in R, x \neq 0$ ist nur in endlich vielen $\mathfrak{p} \in P$ enthalten.

Beispiele für Krullringe sind offensichtlich jeder diskrete Bewertungsring, aber auch jeder Hauptidealring. Außerdem sind für jeden Krullring $R$ auch der Polynomring $R[X]$ und der Ring $R[[X]]$ der formalen Potenzreihen Krullringe.

Nicht jeder Krullring ist noethersch. Umgekehrt lässt sich für einen noetherschen Ring sehr einfach überprüfen, wann er ein Krullring ist:

Lemma 4.5.7 ([Bou72, VII, §1.3, Corollary]). Ein noetherscher Ring ist genau dann ein Krullring, wenn er ganzabgeschlossen ist.

Damit gelten die folgenden Aussagen über Krullringen insbesondere auch über allen ganzabgeschlossenen noetherschen Ringen.

Über einem Krullring lässt sich lokal testen, ob ein Gitter ganz ist. Sei dazu auch hier wieder $(V, q)$ ein endlich-dimensionaler Vektorraum über dem Quotientenkörper $K=$ Quot $(R)$ mit nichtausgearteter quadratischer Form.

Lemma 4.5.8. Sei $R$ ein Krullring. Dann ist ein Gitter $L$ in $(V, q)$ genau dann ganz über $R$, wenn für jedes Primideal $\mathfrak{p} \subset R$ von Höhe 1 das lokalisierte Gitter $L_{\mathfrak{p}}$ über $R_{\mathfrak{p}}$ ganz ist.

Es gilt auch, dass $L$ genau dann ganz ist, wenn für jedes maximale Ideal $\mathfrak{m} \subset R$ das $R_{\mathfrak{m}}$-Gitter $L_{\mathfrak{m}}$ ganz ist.

BEwEIS. Ist $L$ ganz, so ist auch jede Lokalisierung ganz. Ist umgekehrt jede Lokalisierung in den Primidealen von Höhe 1 ganz, so gilt für jedes Element $x \in L$ auch $x \in L_{\mathfrak{p}}$ für jedes Primideal $\mathfrak{p}$ von Höhe 1 und damit auch

$$
q(x) \in \bigcap_{\substack{\mathfrak{p} \subset R \\ \text { ht } \mathfrak{p}=1}} R_{\mathfrak{p}}=R .
$$

Es liege $r$ im Durchschnitt der Lokalisierungen $R_{\mathfrak{m}}$ aller maximalen Ideale $\mathfrak{m}$ von $R$. Sei $\mathfrak{p}$ ein Primideal von Höhe 1 , dann existiert ein maximales Ideal $\mathfrak{m}^{\prime}$ mit $\mathfrak{p} \subseteq \mathfrak{m}^{\prime}$. Folglich gilt $r \in R_{\mathfrak{m}^{\prime}} \subseteq R_{\mathfrak{p}}$. Da dies für jedes $\mathfrak{p}$ gilt, folgt

$$
\bigcap_{\substack{\mathfrak{m} \subset R \\ \max .}} R_{\mathfrak{m}} \subseteq \bigcap_{\substack{\mathfrak{p} \subset R \\ \text { ht } \mathfrak{p}=1}} R_{\mathfrak{p}}=R .
$$

Die zweite Aussage folgt damit aus der ersten. 
Sei vorerst $R$ wieder ein beliebiger Integritätsbereich.

Für einen $R$-Modul $M$ bezeichne wie üblich $M^{*}=\operatorname{Hom}_{R}(M, R)$ den dualen Modul (bzw. den Dualraum). Es existiert stets eine kanonische Einbettung des Moduls in seinen Doppeldualraum:

$$
\begin{aligned}
M & \longrightarrow M^{* *}=\operatorname{Hom}_{R}\left(M^{*}, R\right) \\
x & \longmapsto(\varphi \mapsto \varphi(x)) .
\end{aligned}
$$

Daher identifizieren wir fortan $M$ mit seinem Bild in $M^{* *}$, also $M \subseteq M^{* *}$. Ist $V$ ein endlich-dimensionaler Vektorraum über einem Körper, so ist dieser Morphismus eine kanonische Isomorphie $V=V^{* *}$. Für endlich erzeugte freie Moduln über Ringen gilt dies ebenso, im Allgemeinen ist dies jedoch nicht der Fall.

Definition. Ein $R$-Modul $M$ heißt reflexiv, wenn er mit seinem Doppeldual übereinstimmt, also wenn $M=M^{* *}$ gilt.

Ist $L$ ein ganzes $R$-Gitter in einem nichtausgearteten quadratischen Raum $(V, q)$ über $K=\operatorname{Quot}(R)$, so ergibt die zugehörige Bilinearform nicht nur eine Einbettung von $L$ in $L^{*}$, sondern auch eine kanonische Isomorphie zwischen dualem Gitter und dualem Modul:

$$
\begin{aligned}
L^{\#} & \cong L^{*}=\operatorname{Hom}_{R}(L, R) \\
x & \longmapsto(y \mapsto\langle x, y\rangle) .
\end{aligned}
$$

Es gilt also $L^{\#}=L^{*}$ und auch $L^{\# \#}=L^{* *}$. Letzteres erhalten wir genauso, wenn wir $L^{\# \#}$ als Teilmenge von $V^{* *}$ mittels der kanonischen Einbettung $V \rightarrow V^{* *}$ auffassen.

Hauptsatz 4.5.9 ([Bou72, VII, §4.2, Thm. 1]). Sei $R$ ein noetherscher ganzabgeschlossener Ring und $L$ ein $R$-Gitter in $(V, q)$. Dann gilt

$$
L^{\#}=\bigcap_{\substack{\mathfrak{p} \subset R \\ \text { ht } \mathfrak{p}=1}} L_{\mathfrak{p}}^{\#} .
$$

Korollar 4.5.10 ([Bou72, VII, §4.2, Corollary]). Unter den Voraussetzungen des Hauptsatzes gilt:

$$
L^{\# \#}=\bigcap_{\substack{\mathfrak{p} \subset R \\ \mathrm{ht} \mathfrak{p}=1}} L_{\mathfrak{p}} .
$$

Die erste Gleichheit können wir auch auf die Teilmengen der ganzen Elemente der dualen Gitter übernehmen:

Lemma 4.5.11. Unter den Voraussetzungen des Hauptsatzes gilt:

$$
L^{(\#)}=\bigcap_{\substack{\mathfrak{p} \subset R \\ \text { ht } \mathfrak{p}=1}} L_{\mathfrak{p}}^{(\#)} .
$$


Beweis. Sei $P$ die Menge der Primideale in $R$ von Höhe 1 . Sei $x \in \bigcap_{\mathfrak{p} \in P} L_{\mathfrak{p}}^{(\#)}$. Dann liegt $x$ auch in $\bigcap_{\mathfrak{p} \in P} L_{\mathfrak{p}}^{\#}=L^{\#}$. Außerdem gilt $q(x) \in \bigcap_{\mathfrak{p} \in P} R_{\mathfrak{p}}=R$ und damit $x \in L^{(\#)}$. Die Umkehrung ist klar.

Die beiden Gitter-Identitäten $L \subseteq L^{\# \#}$ und $L^{\#}=L^{\# \# \#}$ könnten wir auch mit Hilfe der Identifikationen der verschiedenen Dualisierungen zeigen.

Nach den obigen Bemerkungen ist ein Gitter $L$ genau dann reflexiv, wenn es mit seinem Doppeldual übereinstimmt, also wenn $L=L^{\# \#}$ gilt. Insbesondere ist das duale Gitter stets reflexiv.

Für ein reflexives Gitter lässt sich die Maximalität über gewissen Ringen lokal prüfen und jedes maximale Gitter ist reflexiv:

Lemma 4.5.12. Ein Gitter L über einem noetherschen ganzabgeschlossenen Ring $R$ ist genau dann maximal, wenn es reflexiv ist und wenn für jedes Primideal $\mathfrak{p} \subset R$ von Höhe 1 das lokalisierte $R_{\mathfrak{p}}$-Gitter $L_{\mathfrak{p}}$ maximal ist.

BeweIs. Ist $L$ reflexiv und jedes $L_{\mathfrak{p}}$ maximal, so gilt $L^{\# \#}=L$ und $L_{\mathfrak{p}}^{(\#)}=L_{\mathfrak{p}}$ und damit

$$
L^{(\#)}=\bigcap_{\substack{\mathfrak{p} \subset R \\ \text { ht } \mathfrak{p}=1}} L_{\mathfrak{p}}^{(\#)}=\bigcap_{\substack{\mathfrak{p} \subset R \\ \mathrm{ht} \mathfrak{p}=1}} L_{\mathfrak{p}}=L^{\# \#}=L .
$$

Ist umgekehrt $L$ ein maximales Gitter, so gilt $L=L^{(\#)}$. Da dann auch alle

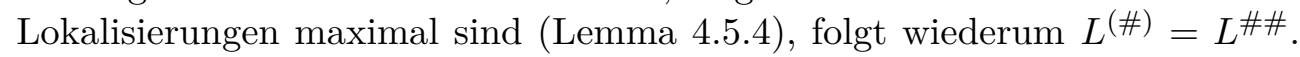

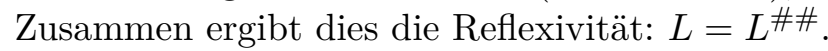

\subsubsection{Maximalordnungen über Krullringen}

Satz 4.5.13. Sei $(C, N)$ eine Kompositionsalgebra über dem Quotientenkörper $K=\operatorname{Quot}(R)$ eines noetherschen Integritätsbereiches $R$. Dann gilt:

1. Sei $B$ eine Maximalordnung in $(C, N)$ über $R$. Dann ist für jedes Primideal $\mathfrak{q} \subset R$ das lokalisierte Gitter $B_{\mathfrak{q}}$ eine Maximalordnung über $R_{\mathfrak{q}}$.

2. Eine R-Ordnung $B$ ist genau dann eine Maximalordnung in $(C, N)$, wenn für jedes maximale Ideal $\mathfrak{m} \subset R$ das lokalisierte Gitter $B_{\mathfrak{m}}$ eine Maximalordnung über $R_{\mathfrak{m}}$ ist.

3. Sei $R$ zusätzlich ganzabgeschlossen. Dann ist ein $R$-Gitter $B$ genau dann eine Maximalordnung in $(C, N)$, wenn $B$ als Modul reflexiv ist und wenn für jedes Primideal $\mathfrak{p} \subset R$ von Höhe 1 das lokalisierte Gitter $B_{\mathfrak{p}}$ eine Maximalordnung über $R_{\mathfrak{p}}$ ist.

BEweIs. Ist $B$ eine Ordnung, so ist offensichtlich auch $B_{\mathfrak{q}}$ für jedes Primideal $\mathfrak{q} \subset R$ eine Ordnung über $R_{\mathfrak{q}}$. Nach Satz 4.2.8 ist eine Ordnung genau dann eine Maximalordnung, wenn sie als Gitter maximal ist. 
Damit folgen der erste und der zweite Teil aus Lemma 4.5.4 bzw. aus Korollar 4.5.6.

Ist $B$ eine Maximalordnung über einem ganzabgeschlossenen noetherschen Ring, so folgt aus dem letzten Lemma, dass $B$ reflexiv ist und auch alle Lokalisierungen maximale Gitter und damit Maximalordnungen sind.

Sind umgekehrt die Lokalisierungen in den Primidealen von Höhe 1 Maximalordnungen und ist $B$ reflexiv, so ist $B$ ein maximales Gitter. Nach Lemma 4.2.10 ist ein maximales Gitter genau dann eine Maximalordnung, wenn $e \in B$ und $\langle x y, z\rangle \in R$ für alle $x, y, z \in B$ gilt. Der zweite Teil folgt hier für alle $x, y, z \in B$ daraus, dass Entsprechendes für alle $B_{\mathfrak{p}}$ gilt und dass $R$ der Durchschnitt seiner Lokalisierungen $R_{\mathfrak{p}}$ ist. Außerdem ist die Eins in $B$ enthalten: $e \in \bigcap_{\mathfrak{p} \in P} B_{\mathfrak{p}}=B^{\# \#}=B$.

Wenden wir auf die letzte Charakterisierung noch die Bijektion von Maximalordnungen über diskreten Bewertungsringen und über ihren Vervollständigungen an, so erhalten wir:

Satz 4.5.14. Sei $(C, N)$ eine Kompositionsalgebra über dem Quotientenkörper $K=\operatorname{Quot}(R)$ eines ganzabgeschlossenen noetherschen Integritätsbereiches $R$.

Dann ist ein R-Gitter $B$ genau dann eine Maximalordnung in $(C, N)$, wenn $B$ reflexiv ist und wenn für jedes Primideal $\mathfrak{p} \subset R$ von Höhe 1 das Gitter $\hat{B}_{\mathfrak{p}}=\hat{R}_{\mathfrak{p}} \otimes_{R} B$ eine Maximalordnung über $\hat{R}_{\mathfrak{p}}$ in der Kompositionsalgebra $\left(\hat{C}_{\mathfrak{p}}=\hat{K}_{\mathfrak{p}} \otimes_{K} C, \hat{N}_{\mathfrak{p}}\right)$ über $\hat{K}_{\mathfrak{p}}$ ist.

Dabei bezeichnen $\hat{R}_{\mathfrak{p}}$ und $\hat{K}_{\mathfrak{p}}$ die Vervollständigungen von $R$ bzw. K bezüglich der durch $\mathfrak{p}$ gegebenen diskreten Bewertung.

Beweis. Es gilt $\hat{B}_{\mathfrak{p}}=\hat{R}_{\mathfrak{p}} \otimes_{R} B=\hat{R}_{\mathfrak{p}} \otimes_{R_{\mathfrak{p}}} B_{\mathfrak{p}}$ und wir können $\hat{B}_{\mathfrak{p}}$ als Vervollständigung des lokalisierten $R_{\mathfrak{p}}$-Gitters $B_{\mathfrak{p}}$ betrachten. Damit folgt die Behauptung aus dem vorhergehenden Satz und aus Lemma 4.4.10.

Über beliebigen Krullringen erhalten wir zumindest ein hinreichendes Kriterium:

Lemma 4.5.15. Sei $(C, N)$ eine Kompositionsalgebra über dem Quotientenkörper $K=\operatorname{Quot}(R)$ eines Krullringes $R$ und sei $B$ ein Gitter in $(C, N)$.

Ist $B$ reflexiv und ist für jedes Primideal $\mathfrak{p} \subset R$ von Höhe 1 das lokalisierte Gitter $B_{\mathfrak{p}}$ eine Maximalordnung über $R_{\mathfrak{p}}$, so ist $B$ eine Maximalordnung über $R$.

BeweIs. Ist $R$ ein beliebiger Krullring, so muss ein Gitter $L$ nicht endlich erzeugt sein und es ist nicht klar, ob das duale Gitter einer Lokalisierung gleich der Lokalisierung des dualen Gitters ist. Für die Schnitte dieser beiden 
Varianten gelten zumindest die folgenden Identitäten:

$$
\begin{aligned}
L^{\#} & =\bigcap_{\substack{\mathfrak{p} \subset R \\
\text { ht } \mathfrak{p}=1}}\left(L^{\#}\right)_{\mathfrak{p}}=\bigcap_{\substack{\mathfrak{p} \subset R \\
\text { ht } \mathfrak{p}=1}}\left(L_{\mathfrak{p}}\right)^{\#}, \quad L^{\# \#} \supseteq \bigcap_{\substack{\mathfrak{p} \subset R \\
\text { ht } \mathfrak{p}=1}} L_{\mathfrak{p}}, \\
L^{(\#)} & =\bigcap_{\substack{\mathfrak{p} \subset R \\
\text { ht } \mathfrak{p}=1}}\left(L^{(\#)}\right)_{\mathfrak{p}}=\bigcap_{\substack{\mathfrak{p} \subset R \\
\text { ht } \mathfrak{p}=1}}\left(L_{\mathfrak{p}}\right)^{(\#)} .
\end{aligned}
$$

Die Gleichung für $L^{\#}$ folgt wie bei Bourbaki für ganzabgeschlossene noethersche Ringe: Zum einen ist $L^{\#} \subseteq\left(L^{\#}\right)_{\mathfrak{p}} \subseteq\left(L_{\mathfrak{p}}\right)^{\#}$ für jedes Primideal $\mathfrak{p} \subset R$ von Höhe 1. Sei umgekehrt $z \in \bigcap_{\mathfrak{p} \in P}\left(L_{\mathfrak{p}}\right)^{\#}$. Dann gilt für jedes $x \in L$ auch $x \in L_{\mathfrak{p}}$ (für jedes $\mathfrak{p}$ ) und damit $\langle x, z\rangle \in \bigcap_{\mathfrak{p} \in P} R_{\mathfrak{p}}=R$. Es folgt $z \in L^{\#}$ und somit die erste Identität. Die Gleichung für $L^{(\#)}$ folgt wie zuvor aus der ersten Gleichung und der Gleichheit $\bigcap_{\mathfrak{p} \in P} R_{\mathfrak{p}}=R$. Anwenden der ersten Gleichung auf das Gitter $L^{\#}$ ergibt zusammen mit der Inklusion $\left(L^{\#}\right)_{\mathfrak{p}} \subseteq\left(L_{\mathfrak{p}}\right)^{\#}$ die noch fehlende Identität:

$$
L^{\# \#}=\bigcap_{\substack{\mathfrak{p} \subset R \\ \text { ht } \mathfrak{p}=1}}\left(\left(L^{\#}\right)_{\mathfrak{p}}\right)^{\#} \supseteq \bigcap_{\substack{\mathfrak{p} \subset R \\ \text { ht } \mathfrak{p}=1}}\left(L_{\mathfrak{p}}\right)^{\# \#}=\bigcap_{\substack{\mathfrak{p} \subset R \\ \text { ht } \mathfrak{p}=1}} L_{\mathfrak{p}} ;
$$

die letzte Umformung, d.h. $L_{\mathfrak{p}}^{\# \#}=L_{\mathfrak{p}}$, gilt, da $L_{\mathfrak{p}}$ als Gitter über einem diskreten Bewertungsring endlich erzeugt und frei ist.

Sind alle lokalisierten Gitter $L_{\mathfrak{p}}$ maximal und ist $L$ reflexiv, so ist zum einen $L$ ganz nach Lemma 4.5.8 und zum anderen gilt

$$
L^{(\#)}=\bigcap_{\substack{\mathfrak{p} \subset R \\ \text { ht } \mathfrak{p}=1}}\left(L_{\mathfrak{p}}\right)^{(\#)}=\bigcap_{\substack{\mathfrak{p} \subset R \\ \text { ht } \mathfrak{p}=1}} L_{\mathfrak{p}} \subseteq L^{\# \#}=L .
$$

Folglich ist $L$ ein maximales Gitter. Die Übertragung der multiplikativen Abgeschlossenheit und das Enthaltensein der Eins folgen wie zuvor.

\subsubsection{Lokale Konstruktion reflexiver Gitter über Krullringen}

Für die Betrachtung von Garben von Maximalordnungen und ihrer lokalen Natur im nächsten Kapitel benötigen wir zunächst noch die beiden folgenden Aussagen über die Konstruktion reflexiver Moduln über ganzabgeschlossenen noetherschen Ringen, die wir hier ohne Beweis zitieren wollen:

Hauptsatz 4.5.16 ([Bou72, VII, §4.3, Thm.3]). Sei $R$ ein noetherscher ganzabgeschlossener Integritätsbereich und sei $P$ die Menge seiner Primideale von Höhe 1. Sei L ein R-Gitter in einem endlich-dimensionalen Vektorraum V.

1. Sei $M$ ein zweites $R$-Gitter in $V$. Für alle bis auf höchstens endlich viele Primideale $\mathfrak{p} \in P$ gilt dann $M_{\mathfrak{p}}=L_{\mathfrak{p}}$. 
2. Für jedes Primideal $\mathfrak{p} \in P$ sei ein $R_{\mathfrak{p}}$-Gitter $N(\mathfrak{p})$ in $V$ gegeben, wobei $N(\mathfrak{p})=L_{\mathfrak{p}}$ für alle bis auf höchstens endlich viele $\mathfrak{p}$ gelte. Dann ist

$$
N=\bigcap_{\mathfrak{p} \in P} N(\mathfrak{p})
$$

ein reflexives $R$-Gitter in $V$, und es ist das einzige reflexive Gitter $N^{\prime}$, für das $N_{\mathfrak{p}}^{\prime}=N(\mathfrak{p})$ für alle $\mathfrak{p} \in P$ gilt.

Lemma 4.5.17 ([Bou72, VII, §4.3, Lemma 1]). Sei $R$ ein ganzabgeschlossener noetherscher Ring $R$ mit Quotientenkörper K. Seien $\mathfrak{p}$ und $\mathfrak{q}$ zwei Primideale in $R$ so, dass (0) das einzige Primideal ist, welches im Durchschnitt $\mathfrak{p} \cap \mathfrak{q}$ enthalten ist. Dann gilt $\left(M_{\mathfrak{p}}\right)_{\mathfrak{q}}=K \otimes_{R} M$ für jeden torsionsfreien endlich erzeugten $R$-Modul $M$. 



\section{Kapitel 5}

\section{Garben von Oktavenalgebren und von Maximalordnungen}

In diesem Kapitel wollen wir untersuchen, inwieweit sich das Konzept von Garben von Azumaya-Algebren oder allgemeiner von Maximalordnungen auf Kompositionsalgebren übertragen lässt. Da Kompositionsalgebren von konstantem Rang 1 oder 2 kommutativ sind und da (reguläre) Quaternionenalgebren ein Spezialfall von Azumaya-Algebren sind, werden wir uns auf die Oktavenalgebren von Rang 8 beschränken.

Da die Oktavenalgebren tatsächlich nicht assoziativ sind, werden auch die betrachteten Garben von Oktavenalgebren oder Maximalordnungen nichtassoziativ sein. Entsprechend werden wir die Sprechweise von nicht notwendig assoziativen Algebren zu nichtassoziativen Algebren verkürzen.

\subsection{Garben von Oktavenalgebren}

Sei $\left(X, \mathcal{O}_{X}\right)$ ein Schema. Eine Garbe von nichtassoziativen $\mathcal{O}_{X}$-Algebren sei ein $\mathcal{O}_{X}$-Modul $\mathcal{F}$ zusammen mit einem Garbenmorphismus $\mu: \mathcal{F} \times \mathcal{F} \rightarrow \mathcal{F}$ so, dass $(\mathcal{F}(U), \mu(U))$ eine nichtassoziative $\mathcal{O}_{X}(U)$-Algebra für jede offene Menge $U \subseteq X$ ist.

Ist $\mathcal{F}$ eine solche Garbe nichtassoziativer $\mathcal{O}_{X}$-Algebren mit Multiplikation $\mu$, so sind für jeden Punkt $x \in X$ der Halm $\mathcal{F}_{x}$ über dem lokalen Ring $\mathcal{O}_{X, x}$ und die Quotientenalgebra $\mathcal{F}(x):=k(x) \otimes_{\mathcal{O}_{X, x}} \mathcal{F}_{x}$ über dem Restklassenkörper $k(x)=\mathcal{O}_{X, x} / \mathfrak{m}_{x}$ ebenfalls nichtassoziative Algebren mit Multiplikation $\mu_{x}$.

Definition. Eine Garbe von Oktavenalgebren auf einem lokal-noetherschen Schema $\left(X, \mathcal{O}_{X}\right)$ ist eine Garbe $\mathcal{C}$ nichtassoziativer $\mathcal{O}_{X^{-}}$Algebren, die als $\mathcal{O}_{X^{-}}$ Modul kohärent ist, zusammen mit einem Garbenmorphismus $N: \mathcal{C} \rightarrow \mathcal{O}_{X}$ derart, dass in jedem abgeschlossenen Punkt $x \in X$ der Halm $\mathcal{C}_{x}$ eine Oktavenalgebra mit Norm $N_{x}$ über dem lokalen Ring $\mathcal{O}_{X, x}$ ist. 
Lemma 5.1.1. Sei $(\mathcal{C}, N)$ eine Garbe von Oktavenalgebren auf einem lokalnoetherschen Schema $\left(X, \mathcal{O}_{X}\right)$. Dann ist $\mathcal{C}$ als $\mathcal{O}_{X}$-Modul lokalfrei und von Rang 8. Außerdem ist $\left(\mathcal{C}_{x}, N_{x}\right)$ für jeden Punkt $x \in X$ eine Oktavenalgebra über dem lokalen Ring $\mathcal{O}_{X, x}$.

Beweis. Sei $x \in X$ ein beliebiger Punkt. Dann enthält sein topologischer Abschluss wenigstens einen abgeschlossenen Punkt $z$. Für diesen ist $\mathcal{O}_{X, x}$ eine flache $\mathcal{O}_{X, z}$-Algebra, sodass mit $\mathcal{C}_{z}$ über $\mathcal{O}_{X, z}$ auch $\mathcal{C}_{x}=\mathcal{O}_{X, x} \otimes_{\mathcal{O}_{X, z}} \mathcal{C}_{z}$ eine Oktavenalgebra über $\mathcal{O}_{X, x}$ ist. Insbesondere ist für jedes $x \in X$ der Halm $\mathcal{C}_{x}$ als projektiver Modul über einem lokalen Ring frei und von Rang 8. Damit ist $\mathcal{C}$ lokalfrei (siehe etwa [Har77, Ex. II.5.7] oder [Mil80, Theorem I.2.9]) und von konstantem Rang 8.

Lemma 5.1.2. Sei $X=\operatorname{Spec} R$ ein affines noethersches Schema. Dann ist eine kohärente Garbe $\mathcal{C}$ nichtassoziativer $\mathcal{O}_{X}$-Algebren zusammen mit einem Garbenmorphismus $N: \mathcal{C} \rightarrow \mathcal{O}_{X}$ genau dann eine Garbe von Oktavenalgebren, wenn $(\Gamma(X, \mathcal{C}), N(X))$ eine Oktavenalgebra über $R$ ist.

BeweIs. Es ist $R$ ein noetherscher Ring. Die kohärenten $\mathcal{O}_{X}$-Moduln $\mathcal{M}$ auf $X=\operatorname{Spec} R$ entsprechen den endlich erzeugten $R$-Moduln $M$ (via $\mathcal{M} \mapsto$ $\Gamma(X, \mathcal{M})$ bzw. $M \mapsto \widetilde{M})$. Dabei gilt $\widetilde{M}_{x}=M_{x}$ für $x \in X$, und Garbenmorphismen $\mu: \mathcal{M} \times \mathcal{M} \rightarrow \mathcal{M}$ bzw. $N: \mathcal{M} \rightarrow \mathcal{O}_{X}$ entsprechen $R$-bilinearen Abbildungen $M \times M \rightarrow M$ bzw. Abbildungen $M \rightarrow R$. Sei nun $C=\Gamma(X, \mathcal{C})=\mathcal{C}(X)$. Dann ist $(\mathcal{C}, N)$ genau dann eine Garbe von Oktavenalgebren, wenn für jedes abgeschlossene $x \in X$ der Halm $\mathcal{C}_{x}=C_{x}$ eine Oktavenalgebra über $\mathcal{O}_{X, x}=R_{x}$ mit Norm $N_{x}$ ist. Dies ist genau dann der Fall, wenn $C$ eine Oktavenalgebra über $R$ mit Norm $N(X)$ ist (siehe Lemma 3.3.4 - über einem noetherschen Ring ist jeder endlich erzeugte Modul endlich präsentiert).

Folglich ist in einer Garbe $(\mathcal{C}, N)$ von Oktavenalgebren über einem beliebigen lokal-noetherschen Schema $\left(X, \mathcal{O}_{X}\right)$ die Algebra $(\mathcal{C}(U), N(U))$ eine Kompositionsalgebra über $\mathcal{O}_{X}(U)$ für jede offene affine Menge $U \subseteq X$.

Warnung: Über einer offenen nicht-affinen Menge $U \subseteq X$ ist $(\mathcal{C}(U), N(U))$ zwar eine nichtassoziative Algebra mit multiplikativer nichtausgearteter quadratischer Form, als $\mathcal{O}_{X}(U)$-Modul muss sie jedoch nicht notwendigerweise projektiv sein.

Ist $\mathcal{A}$ eine Garbe von Azumaya-Algebren auf $\left(X, \mathcal{O}_{X}\right)$, so ist in jedem Punkt $x \in$ $X$ die Quotientenalgebra $\mathcal{A}(x):=k(x) \otimes \mathcal{A}_{x}$ eine Azumaya-Algebra über dem Restklassenkörper $k(x)=\mathcal{O}_{X, x} / \mathfrak{m}_{x}$. Diese Eigenschaft gilt für Oktavenalgebren über Ringen im Allgemeinen nicht - wir haben explizit schlechte Reduktion, also das Ausarten der Bilinearform modulo Primidealen, erlaubt. Entsprechend werden bei der hier gewählten Definition von Garben von Oktavenalgebren im Allgemeinen nicht alle Quotientenalgebren Oktavenalgebren sein. 
Definition. Eine Garbe $\mathcal{C}$ von Oktavenalgebren hat gute Reduktion in einem Punkt $x \in X$, wenn $\mathcal{C}(x):=k(x) \otimes \mathcal{C}_{x}$ eine Oktavenalgebra über dem Restklassenkörper $k(x)$ ist.

Eine Garbe von Oktavenalgebren mit guter Reduktion ist eine Garbe von Oktavenalgebren, die in jedem abgeschlossenen Punkt gute Reduktion hat.

Andernfalls sprechen wir von schlechter Reduktion in Punkten oder einer Menge von Punkten von $X$.

Lemma 5.1.3. Sei $(\mathcal{C}, N)$ eine Garbe von Oktavenalgebren auf einem lokalnoetherschen Schema $\left(X, \mathcal{O}_{X}\right)$. Dann hat $\mathcal{C}$ genau dann gute Reduktion in $x \in X$, wenn die Oktavenalgebra $\left(\mathcal{C}_{x}, N_{x}\right)$ über dem lokalen Ring $\mathcal{O}_{X, x}$ als quadratischer Modul regulär ist. Hat $\mathcal{C}$ gute Reduktion in den abgeschlossenen Punkten, so hat sie gute Reduktion in allen Punkten.

BeweIs. Die Regularität von $\left(\mathcal{C}_{x}, N_{x}\right)$ in einem Punkt $x$ guter Reduktion folgt aus Lemma 3.3.5, ebenso wie die Umkehrung der Aussage. Außerdem überträgt sich die Regularität auf die Algebra $\left(\mathcal{C}_{y}=\mathcal{O}_{X, y} \otimes_{\mathcal{O}_{X, x}} \mathcal{C}_{x}, N_{y}\right)$ für jeden Punkt $y$, in dessen Abschluss $x$ liegt.

\subsection{Garben von Maximalordnungen}

Im Allgemeinen kann es schwierig sein, Garben von Oktavenalgebren auf einem gegebenen noetherschen Schema $X$ zu finden. Wie auch schon bei den Azumaya-Algebren wollen wir die Definition aufweichen und sogenannte Verzweigungsorte erlauben, in denen eine Garbe nicht alle Bedingungen einer Oktavengarbe erfüllt. Dazu verallgemeinern wir das Konzept von Ordnungen in Azumaya-Algebren auf die hier betrachteten nichtassoziativen Oktavenalgebren. Wir folgen in der Definition Milnes assoziativer Variante, siehe [Mil80, Ch. IV, Beweis von Lemma 2.17, Seite 151].

Definition. Sei $\left(X, \mathcal{O}_{X}\right)$ ein ganzes, lokal-noethersches Schema und sei $(C, N)$ eine Oktavenalgebra über dem Funktionenkörper $k(X)$. Eine nichtassoziative Ordnung in $(C, N)$ ist eine kohärente Garbe $\mathcal{A}$ nichtassoziativer $\mathcal{O}_{X}$-Algebren so, dass der Halm $\mathcal{A}_{x}$ für jedes $x \in X$ eine $\mathcal{O}_{X, x^{-}}$Ordnung in $(C, N)$ ist.

Eine Maximalordnung ist eine nichtassoziative Ordnung, die in keiner nichtassoziativen Ordnung echt enthalten ist.

Ist $\mathcal{A}$ eine nichtassoziative Ordnung in $(C, N)$, so ist für jeden Punkt $x \in X$ der Halm $\mathcal{A}_{x}$ als $\mathcal{O}_{X, x}$-Ordnung in $(C, N)$ insbesondere torsionsfrei. Daher ist auch für jede offene affine Menge $U=\operatorname{Spec} R$ in $X$ die $\mathcal{O}(U)$-Algebra $\mathcal{A}(U)$ torsionsfrei (da $X$ ganz ist, ist jede offene affine Menge zusammenhängend). Außerdem gilt für den generischen Punkt $\eta \in X$ insbesondere $\mathcal{A}_{\eta}=C$. Diese letzte Eigenschaft ist zusammen mit der Torsionsfreiheit die wesentliche Bedingung an eine Ordnung: 
Lemma 5.2.1. Sei $\left(X, \mathcal{O}_{X}\right)$ ein ganzes, lokal-noethersches Schema und sei $(C, N)$ eine Oktavenalgebra über $k(X)$. Dann gilt:

1. Ist $X$ regulär oder noethersch, so ist jede nichtassoziative Ordnung torsionsfrei.

2. Ist $X$ normal und ist $\mathcal{A}$ eine torsionsfreie, kohärente Garbe nichtassoziativer $\mathcal{O}_{X}$-Algebren mit generischer Faser $\mathcal{A}_{\eta}=C$, so ist $\mathcal{A}$ eine nichtassoziative Ordnung in $(C, N)$.

BeweIs. Für jedes $x \in X$ ist der Halm $\mathcal{A}_{x}$. Ist $X$ regulär oder noethersch, so ist dann auch $\mathcal{A}$ selbst torsionsfrei, siehe Sätze B.1.6 und B.1.7.

Ist $\mathcal{A}_{\eta}=C$, so gilt für jedes $x \in X$ auch $\mathcal{O}_{X, \eta} \otimes_{\mathcal{O}_{X, x}} \mathcal{A}_{x}=\mathcal{A}_{\eta}$. Ist $\mathcal{A}$ torsionsfrei, so ist für jedes $x \in X$ die kanonische Abbildung $\mathcal{A}_{x} \rightarrow \mathcal{O}_{X, \eta} \otimes_{\mathcal{O}_{X, x}} \mathcal{A}_{x}$ eine Einbettung. Also ist $\mathcal{A}_{x}$ ein multiplikativ abgeschlossenes Gitter in $C$. Ist $X$ normal, so ist $\mathcal{O}_{X, x}$ ganzabgeschlossen und $\mathcal{A}_{x}$ ist nach Lemma 4.2.2 ein ganzes Gitter und damit eine $\mathcal{O}_{X, x^{-}}$Ordnung in $(C, N)$.

Lemma 5.2.2. Sei $X$ ein ganzes, lokal-noethersches Schema und sei $(C, N)$ eine Oktavenalgebra über $k(X)$. Sei $\mathcal{A}$ eine nichtassoziative Ordnung in $(C, N)$ und sei $U=\operatorname{Spec} R$ eine offene affine Menge in $X$, deren Ring $R$ ganzabgeschlossen ist. Dann ist auch $\mathcal{A}(U)$ eine $\mathcal{O}_{X}(U)$-Ordnung in $(C, N)$.

BEwEIS. Offensichtlich gilt $\mathcal{O}_{X, \eta} \otimes_{\mathcal{O}_{X}(U)} \mathcal{A}(U)=\mathcal{A}_{\eta}=C$. Da $\mathcal{A}(U)$ torsionsfrei ist, lässt es sich in $\mathcal{O}_{X, \eta} \otimes \mathcal{A}(U)=C$ einbetten und ist somit ein Gitter in $C$. Ist $\mathcal{O}(U)$ ganzabgeschlossen, so folgt wiederum aus Lemma 4.2.2, dass $\mathcal{A}(U)$ eine Ordnung in $(C, N)$ ist.

Da bereits für assoziative Ordnungen Artin und de Jong ([AdJ, Ch. 1.8 Orders over schemes]) es für sinnvoll erachten, nur normale Schemata zu betrachten, wollen auch wir uns fortan auf solche Schemata beschränken.

Sei $\left(X, \mathcal{O}_{X}\right)$ ein normales, ganzes, lokal-noethersches Schema und sei $\mathcal{A}$ eine nichtassoziative Ordnung in einer Oktavenalgebra $(C, N)$ über $k(X)$. Dann ist für jeden Punkt $x \in X$ der lokale Ring $\mathcal{O}_{X, x}$ und damit auch für jede offene affine Menge $U=\operatorname{Spec} R \subseteq X$ der Ring $\mathcal{O}_{X}(U)=R$ ein ganzabgeschlossener noetherscher Integritätsbereich, also jeweils ein Krullring. Insbesondere ist dann nach dem obigen Lemma die Algebra $\mathcal{A}(U)$ eine $R$-Ordnung in $(C, N)$.

Im Folgenden wollen wir untersuchen, inwieweit man auch für Garben lokal testen kann, ob eine Ordnung eine Maximalordnung ist.

Lemma 5.2.3. Sei $\left(X, \mathcal{O}_{X}\right)$ ein normales, ganzes, lokal-noethersches Schema und sei $\mathcal{A}$ eine nichtassoziative Ordnung in einer Oktavenalgebra $(C, N)$ über $k(X)$. Ist für jede offene affine Menge $U=\operatorname{Spec} R$ in $X$ die Algebra $\mathcal{A}(U)$ eine Maximalordnung über $\mathcal{O}(U)=R$, so ist für jeden Punkt $x \in X$ der Halm $\mathcal{A}_{x}$ eine Maximalordnung über $\mathcal{O}_{X, x}$ und auch $\mathcal{A}$ selbst ist eine Maximalordnung in $(C, N)$. 
Beweis. Jeder Punkt $x \in X$ besitzt eine offene affine Umgebung $U=\operatorname{Spec} R$ mit einem noetherschen Ring $R$. Daher folgt die Behauptung aus dem entsprechenden Satz 4.5.13 (1) für Maximalordnungen über noetherschen Ringen. Sei $\mathcal{B}$ eine Ordnung in $(C, N)$, die $\mathcal{A}$ enthalte. Dann existiert eine Inklusion $\varphi: \mathcal{A} \rightarrow \mathcal{B}$. Da diese in jedem Punkt $x \in X$ ein Isomorphismus ist $-\mathcal{A}_{x}$ ist ja eine Maximalordnung -, muss auch $\varphi$ selbst ein Isomorphismus sein und es gilt $\mathcal{A}=\mathcal{B}$.

Ist eine nichtassoziative Ordnung lokal in jedem Punkt eine Maximalordnung, so ist sie auch selbst maximal:

Lemma 5.2.4. Sei $\left(X, \mathcal{O}_{X}\right)$ ein normales, ganzes, lokal-noethersches Schema und sei $\mathcal{A}$ eine nichtassoziative Ordnung in einer Oktavenalgebra $(C, N)$ über $k(X)$. Es gelte eine der beiden Bedingungen:

1. Sei für jeden abgeschlossenen Punkt $x \in X \operatorname{der}$ Halm $\mathcal{A}_{x}$ eine Maximalordnung über dem lokalen Ring $\mathcal{O}_{X, x}$.

2. Sei $\mathcal{A}$ als $\mathcal{O}_{X}$-Modul reflexiv und sei für jeden Punkt $x \in X^{(1)}$ von Kodimension 1 der Halm $\mathcal{A}_{x}$ eine Maximalordnung über $\mathcal{O}_{X, x}$.

Dann ist $\mathcal{A}$ eine Maximalordnung in $(C, N)$ und für jede offene affine Menge $U=\operatorname{Spec} R$ in $X$ ist die Algebra $\mathcal{A}(U)$ eine Maximalordnung über $\mathcal{O}(U)=R$.

Beweis. Sei $U=\operatorname{Spec} R \subseteq X$ eine offene affine Menge. Dann ist $R$ ganzabgeschlossen und noethersch und aus Satz 4.5.13 folgt in beiden Fällen, dass $\mathcal{A}(U)$ eine Maximalordnung über $R$ in $(C, N)$ ist. Der andere Teil der Behauptung folgt damit aus dem vorherigen Lemma.

Es gilt auch die Umkehrung. Wir behandeln zuerst den Fall eines affinen Schemas. Dieser ergibt sich direkt aus den entsprechenden Aussagen über Ringen:

Lemma 5.2.5. Sei $\left(X, \mathcal{O}_{X}\right)$ ein affines, normales, ganzes, lokal-noethersches Schema und sei $\mathcal{A}$ eine nichtassoziative Maximalordnung in einer Oktavenalgebra $(C, N)$ über $k(X)$. Dann ist für jeden Punkt $x \in X$ der Halm $\mathcal{A}_{x}$ eine Maximalordnung über dem lokalen Ring $\mathcal{O}_{X, x}$.

BeweIs. Es ist $X=\operatorname{Spec} R$ für einen ganzabgeschlossenen noetherschen Integritätsbereich $R$. Sei $A=\mathcal{A}(X)$ die Algebra der globalen Schnitte von $\mathcal{A}$; nach Lemma 5.2.2 ist $A$ eine Ordnung über $R$ in $(C, N)$. Als kohärente Garbe ist $\mathcal{A}$ die zu $A$ assoziierte $\mathcal{O}_{X}$-Modulgarbe $\widetilde{A}$ und insbesondere gilt $\mathcal{A}_{x}=A_{x}$ für jeden Punkt $x \in X$. Angenommen, es wäre $\mathcal{A}_{x}=A_{x}$ keine Maximalordnung in einem Punkt $x \in X$. Dann folgt aus Satz 4.5.13, dass auch $A$ keine Maximalordnung über $R$ ist. Es existiert daher eine größere Ordnung $A^{\prime}$, die $A$ echt enthält. Insbesondere definiert deren assoziierte Garbe $\mathcal{A}^{\prime}=\widetilde{A^{\prime}}$ somit eine größere nichtassoziative Ordnung auf $X$ in $(C, N)$, die $\mathcal{A}$ echt enthält, im Widerspruch zur Maximalität von $\mathcal{A}$. 
Auch über nicht-affinen Schemata gilt diese Umkehrung:

Satz 5.2.6. Sei $\left(X, \mathcal{O}_{X}\right)$ ein normales, ganzes, lokal-noethersches Schema und sei $\mathcal{A}$ eine nichtassoziative Maximalordnung in einer Oktavenalgebra $(C, N)$ über $k(X)$. Dann ist für jeden Punkt $x \in X \operatorname{der~Halm~} \mathcal{A}_{x}$ eine Maximalordnung über dem lokalen Ring $\mathcal{O}_{X, x}$.

Beweis. Angenommen, es wäre in einem Punkt $x \in X$ die lokale Algebra $\mathcal{A}_{x}$ keine Maximalordnung in $(C, N)$. Sei $U_{0}$ eine offene affine Umgebung von $x$. Wie im vorhergehenden Beweis eines affinen Schemas folgt, dass die nichtassoziative $\mathcal{O}_{X}\left(U_{0}\right)$-Algebra $A:=\mathcal{A}\left(U_{0}\right)$ eine Ordnung in $(C, N)$ ist, die nicht maximal ist. Sei $A^{\prime}$ eine $\mathcal{O}_{X}\left(U_{0}\right)$-Ordnung, die $A$ echt enthält. Wir definieren für die Punkte $\mathfrak{p} \in X^{(1)}$ von Kodimension 1 die $\mathcal{O}_{X, \mathfrak{p}}$ Ordnungen

$$
B_{\mathfrak{p}}:= \begin{cases}A_{\mathfrak{p}}^{\prime} & \text { für } \mathfrak{p} \in U_{0} \cap X^{(1)} \\ \mathcal{A}_{\mathfrak{p}} & \text { für } \mathfrak{p} \in X^{(1)} \backslash U_{0},\end{cases}
$$

und es sei $\mathcal{B}$ diejenige Garbe auf $X$, die durch die Festlegung

$$
\mathcal{B}(U):=\bigcap_{\mathfrak{p} \in U \cap X^{(1)}} B_{\mathfrak{p}}
$$

für offene Mengen $U$ in $X$ gegeben ist (offensichtlich ist $\mathcal{B}$ tatsächlich eine Garbe).

Da $\mathcal{A}$ kohärent ist, gilt $\mathcal{A}_{\mathfrak{p}}=\mathcal{A}(V)_{\mathfrak{p}}$ für jede offene affine Menge $V \subset X$ und alle $\mathfrak{p} \in V$, und damit ist

$$
\mathcal{B}(V)=\bigcap_{\mathfrak{p} \in V \cap X^{(1)}} B_{\mathfrak{p}} \supseteq \bigcap_{\mathfrak{p} \in V \cap X^{(1)}} \mathcal{A}_{\mathfrak{p}}=\bigcap_{\mathfrak{p} \in V \cap X^{(1)}} \mathcal{A}(V)_{\mathfrak{p}}=\mathcal{A}(V)^{\# \#} \supseteq \mathcal{A}(V) .
$$

Da $\mathcal{A}$ und $\mathcal{B}$ Garben sind, gilt damit auch $\mathcal{B}(U) \supseteq \mathcal{A}(U)$ für jede beliebige offene Teilmenge $U$ von $X$. Außerdem gilt speziell auf der offenen Menge $U_{0}$ :

$$
\mathcal{B}\left(U_{0}\right)=\bigcap_{\mathfrak{p} \in U_{0} \cap X^{(1)}} A_{\mathfrak{p}}^{\prime}=\left(A^{\prime}\right)^{\# \#} \supseteq A^{\prime} \supsetneq A=\mathcal{A}\left(U_{0}\right) .
$$

Folglich ist $\mathcal{B}$ eine Garbe, die $\mathcal{A}$ echt enthält: $\mathcal{B} \supsetneq \mathcal{A}$.

Sei $V$ eine offene affine Teilmenge von $X$. Nach Hauptsatz 4.5.16 ist $\mathcal{B}(V)$ ein reflexives $\mathcal{O}_{X}(V)$-Gitter in $(C, N)$ und somit insbesondere ein endlich erzeugter $\mathcal{O}_{X}(V)$-Modul. Da $\mathcal{B}$ und $\mathcal{O}_{X}$ Garben sind, ist auch für jede beliebige offene Teilmenge $U$ von $X$ die Menge $\mathcal{B}(U)$ ein $\mathcal{O}_{X}(U)$-Modul. Folglich ist $\mathcal{B}$ eine Garbe von $\mathcal{O}_{X}$-Moduln. Als Durchschnitt von Ordnungen ist auch $\mathcal{B}(U)$ multiplikativ abgeschlossen und enthält die Eins für jede offene Teilmenge $U$ von $X$. Somit ist $\mathcal{B}$ eine Garbe nichtassoziativer $\mathcal{O}_{X}$-Algebren.

Sei $\mathfrak{p} \in X^{(1)}$ ein Punkt von Kodimension 1. Erneut nach Hauptsatz 4.5.16 gilt

$$
\mathcal{B}(V)_{\mathfrak{p}}=B_{\mathfrak{p}}
$$


für jede offene affine Umgebung $V$ von $\mathfrak{p}$ und damit auch

$$
\mathcal{B}_{\mathfrak{p}}=B_{\mathfrak{p}} .
$$

Sei $\mathfrak{q} \in X$ ein beliebiger Punkt, und sei $V$ eine offene affine Umgebung von $\mathfrak{q}$. Sei $\mathfrak{p} \in V \cap X^{(1)}$ ein Punkt von Kodimension 1. Ist $\mathfrak{p}-$ als Primideal in $\mathcal{O}_{X}(V)$ betrachtet - in $\mathfrak{q}$ enthalten oder, geometrisch ausgedrückt, liegt der Punkt $\mathfrak{q}$ im Abschluss $\overline{\{\mathfrak{p}\}}$, so gilt für die Lokalisierungen $\left(\mathcal{B}(V)_{\mathfrak{p}}\right)_{\mathfrak{q}}=\left(\mathcal{B}(V)_{\mathfrak{q}}\right)_{\mathfrak{p}}=\mathcal{B}(V)_{\mathfrak{p}}$. Andernfalls ist (0) das einzige Primideal, das im Durchschnitt $\mathfrak{p} \cap \mathfrak{q}$ enthalten ist, und Lemma 4.5 .17 besagt $\left(\mathcal{B}(V)_{\mathfrak{p}}\right)_{\mathfrak{q}}=\left(\mathcal{B}(V)_{\mathfrak{q}}\right)_{\mathfrak{p}}=C$. Insbesondere folgt daraus

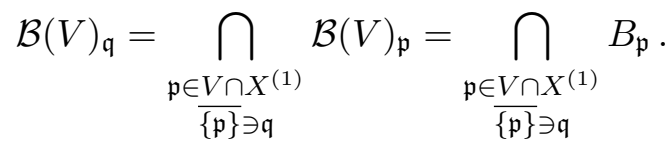

Liegt $\mathfrak{q}$ im Abschluss von $\mathfrak{p}$, so enthält jede offene Umgebung von $\mathfrak{q}$ auch $\mathfrak{p}$, und wir erhalten

$$
\mathcal{B}(V)_{\mathfrak{q}}=\bigcap_{\mathfrak{p} \in X^{(1)}, \overline{\{\mathfrak{p}\} \ni \mathfrak{q}}} B_{\mathfrak{p}}
$$

und somit auch

$$
\mathcal{B}_{\mathfrak{q}}=\bigcap_{\mathfrak{p} \in X^{(1)}, \overline{\{\mathfrak{p}\} \ni \mathfrak{q}}} B_{\mathfrak{p}} \quad \text { und } \quad \mathcal{B}_{\mathfrak{q}}=\mathcal{B}(V)_{\mathfrak{q}}
$$

für jeden Punkt $\mathfrak{q} \in X$ und jede offene affine Umgebung $V$ von $\mathfrak{q}$.

Hieraus folgt, dass auf jeder offenen affinen Teilmenge $V$ von $X$ die Einschränkung $\left.\mathcal{B}\right|_{V}$ der Garbe auf $V$ mit der zu $\mathcal{B}(V)$ assoziierten Modulgarbe übereinstimmt. Daher ist $\mathcal{B}$ quasikohärent. Da jedes $\mathcal{B}(V)$ endlich erzeugt ist, ist $\mathcal{B}$ auch kohärent.

Da für jeden Punkt $\mathfrak{p}$ von Kodimension 1 die lokale Algebra $\mathcal{B}_{\mathfrak{p}}=B_{\mathfrak{p}}$ eine $\mathcal{O}_{\mathfrak{p}^{-}}$ Ordnung in $(C, N)$ ist, folgt aus Lemma 4.5.8, dass auch $\mathcal{B}(V)$ als Gitter ganz und somit eine $\mathcal{O}_{X}(V)$-Ordnung in $(C, N)$ ist für jede offene affine Teilmenge $V$ in $X$. Offensichtlich ist dann auch die lokale Algebra $\mathcal{B}_{\mathfrak{q}}=\mathcal{B}(V)_{\mathfrak{q}}$ eines jeden Punktes $\mathfrak{q} \in V$ eine Ordnung in $(C, N)$ über dem jeweiligen lokalen $\operatorname{Ring} \mathcal{O}_{X, \mathfrak{q}}$. Insgesamt ist die oben konstruierte Garbe $\mathcal{B}$ somit eine kohärente Garbe nichtassoziativer $\mathcal{O}_{X}$-Moduln, deren sämtliche Halme Ordnungen über den entsprechenden lokalen Ringen sind. Damit ist sie eine Ordnung in $(C, N)$, die $\mathcal{A}$ echt enthält, und somit kann $\mathcal{A}$ nicht maximal sein.

Insgesamt erhalten wir, dass wir auch für Garben lokal entscheiden können, wann eine reflexive Ordnung eine Maximalordnung ist:

Satz 5.2.7. Sei $\left(X, \mathcal{O}_{X}\right)$ ein normales, ganzes, lokal-noethersches Schema und sei $\mathcal{A}$ eine reflexive nichtassoziative Ordnung in einer Oktavenalgebra $(C, N)$ über $k(X)$. Dann ist $\mathcal{A}$ genau dann eine Maximalordnung, wenn für jeden 
Punkt $x \in X$ der Halm $\mathcal{A}_{x}$ eine Maximalordnung über dem lokalen Ring $\mathcal{O}_{X, x}$ ist.

Bemerkung 5.2.8 (Vergleich mit assoziativen Maximalordnungen in Azumaya-Algebren). Für assoziative Ordnungen in Azumaya-Algebren zeigen Artin und de Jong, dass sich jede Ordnung $\mathcal{A}^{\prime}$ auf einer nichtleeren offenen Teilmenge $U \subseteq X$, die die Einschränkung einer $X$-Ordnung $\mathcal{B}$ enthält, zu einer $X$-Ordnung $\mathcal{A}$, die $\mathcal{B}$ enthält, fortsetzen lässt, siehe [AdJ, Prop. 1.8.1]. Anders als bei unserem lokalen Vorgehen wird $\mathcal{A}$ bei Artin und de Jong abstrakter als $\mathcal{A}=\operatorname{End}_{\mathcal{O}_{X}}(\mathcal{L}) \cap j_{*} \mathcal{A}^{\prime}$ konstruiert, wobei $j$ die Einbettung von $U$ in $X$ bezeichne und $\mathcal{L}$ eine kohärente Untergarbe von $j_{*} \mathcal{A}^{\prime}$ sei, die $j^{*} \mathcal{L}=\mathcal{A}^{\prime}$ erfüllt und zugleich ein $\mathcal{B}$-Rechtsmodul ist. Auf Ringniveau (mit Einbettung $j: R \rightarrow R^{\prime}$ ) könnte man $L$ als $L=L_{1} B$ konstruieren für ein beliebiges $R$-Gitter $L_{1}$ mit $R^{\prime} L_{1}=A^{\prime}$.

Diese Konstruktion lässt nicht direkt auf die nichtassoziativen Ordnungen übertragen: Zwar wäre auch im nichtassoziativen Fall entsprechend $L_{1} B$ wieder ein $R$-Gitter, jedoch ist im Allgemeinen nicht klar, ob $L_{1} B$ invariant unter Rechtsmultiplikation mit Elementen aus $B$ wäre. Selbst wenn man ein passendes Gitter $L$ findet, so ist auch der zweite Schritt im nichtassoziativen Fall problematisch: Zwar ist die Rechtsmultiplikation mit Elementen aus $A$ ein Vektorraumhomomorphismus auf $C$, im Allgemeinen jedoch nicht multiplikativ. Zudem ist auch die Abbildung, die ein Element $x \in A$ auf die Rechtsmultiplikation mit $x$ abbildet, nicht multiplikativ. Das konstruierte Objekt muss also nicht multiplikativ abgeschlossen sein. - Vergleiche hierzu auch Lemma 4.2.6, in dem wir immerhin zeigen können, dass der Durchschnitt von Rechts- und Linksordnung $O_{r}(L) \cap O_{\ell}(L)$ für gewisse Ringerweiterungen wieder multiplikativ abgeschlossen ist.

\subsubsection{Vergleich von Maximalordnungen und Oktavenalgebren}

Nichtassoziative Ordnungen sind - analog zu den assoziativen Ordnungen in Azumaya-Algebren - eine Verallgemeinerung von Garben von Oktavenalgebren:

Lemma 5.2.9. Sei $\left(X, \mathcal{O}_{X}\right)$ ein normales, ganzes, lokal-noethersches Schema mit generischem Punkt $\eta$. Dann ist jede Garbe $(\mathcal{C}, N)$ von Oktavenalgebren eine nichtassoziative Ordnung in $\left(\mathcal{C}_{\eta}, N_{\eta}\right)$. Hat die Garbe $(\mathcal{C}, N)$ gute Reduktion, so ist sie eine Maximalordnung.

Beweis. Nach Lemma 4.2.11 ist für jedes $x \in X$ der Halm $\mathcal{C}_{x}$ eine Ordnung in $\mathcal{O}_{X, \eta} \otimes \mathcal{C}_{x}=\mathcal{C}_{\eta}$. Als lokalfreie Garbe ist $(\mathcal{C}, N)$ insbesondere torsionsfrei. Hat $(\mathcal{C}, N)$ zusätzlich gute Reduktion in einem Punkt $x \in X$, so ist $\left(\mathcal{C}_{x}, N_{x}\right)$ eine reguläre Kompositionsalgebra und damit eine Maximalordnung in $(C, N)$ nach Lemma 4.2.11. Gilt dies in allen Punkten, so ist nach Lemma 5.2.4 auch die Garbe $(\mathcal{C}, N)$ eine Maximalordnung. 
Umgekehrt gilt, dass eine nichtassoziative Ordnung zumindest lokal in den Punkten der Kodimension 1 eine Oktavenalgebra ist:

Lemma 5.2.10. Sei $\left(X, \mathcal{O}_{X}\right)$ ein ganzes, lokal-noethersches Schema, und sei $(C, N)$ eine Kompositionsalgebra über dem Funktionenkörper $k(X)$. Sei $\mathcal{A}$ eine nichtassoziative Ordnung in $(C, N)$. Dann ist für jeden Punkt $x \in X^{(1)}$ der Kodimension 1 die Ordnung $\mathcal{A}_{x}$ eine - nicht notwendig reguläre - Kompositionsalgebra über $\mathcal{O}_{X, x}$.

Beweis. Für jedes $x \in X$ mit $\operatorname{codim} x=1$ ist $\mathcal{O}_{X, x}$ ein diskreter Bewertungsring, also insbesondere ein Hauptidealring. Damit ist die Ordnung $\mathcal{A}_{x}$ eine Kompositionsalgebra über $\mathcal{O}_{X, x}$ nach Lemma 4.3.1.

Dabei kann es in der Tat vorkommen, dass die Kompositionsalgebra $\mathcal{A}_{x}$ nicht regulär ist, also schlechte Reduktion hat. Wir sagen, dass dann auch $\mathcal{A}$ als Maximalordnung betrachtet schlechte Reduktion in $x$ hat.

\subsection{Beispiel einer Garbe von Oktavenalgebren}

In diesem Abschnitt wollen wir eine nichttriviale Garbe von Oktavenalgebren auf einem geeigneten Schema konstruieren. Da über dem Funktionenkörper $k(x, y)$ der projektiven Ebene alle quadratischen Formen zerfallen, werden wir den dreidimensionalen projektiven Raum betrachten.

Sei $F=k(x, y, z)$ der Körper der rationalen Funktionen in drei Unbestimmten über einem algebraisch abgeschlossenen Körper $k$ von Charakteristik 0.

\subsubsection{Definition einer Quaternionenalgebra}

Es sei $D=(-x,-y)$ die Quaternionenalgebra über dem Körper $F=k(x, y, z)$, die von den zwei Unbestimmten $i$ und $j$ mit Quadraten $-x$ bzw. $-y$ erzeugt wird:

$$
D=F \cdot 1 \oplus F \cdot i \oplus F \cdot j \oplus F \cdot(i j)
$$

mit

$$
i^{2}=-x, \quad j^{2}=-y, \quad j i=-i j .
$$

Unmittelbar ergibt sich das Quadrat des Produktes $i j$ als $(i j)^{2}=-x y$.

Sei $\lambda=\left(\lambda_{1}, \lambda_{2}, \lambda_{3}, \lambda_{4}\right)=\lambda_{1}+\lambda_{2} i+\lambda_{3} j+\lambda_{4} i j \in D$ mit $\lambda_{t} \in F$, dann definiert die Konjugation

$$
\bar{\lambda}=\lambda_{1}-\lambda_{2} i-\lambda_{3} j-\lambda_{4} i j
$$

die Norm

$$
N(\lambda)=\lambda \bar{\lambda}=\lambda_{1}^{2}+\lambda_{2}^{2} x+\lambda_{3}^{2} y+\lambda_{4}^{2} x y .
$$


Diese ist eine multiplikative, quadratische Form auf $D$ mit zugehöriger nichtausgearteter Bilinearform

$$
\langle\lambda, \mu\rangle=2\left(\lambda_{1} \mu_{1}+\lambda_{2} \mu_{2} x+\lambda_{3} \mu_{3} y+\lambda_{4} \mu_{4} x y\right)
$$

für $\lambda$ wie zuvor und $\mu=\mu_{1}+\mu_{2} i+\mu_{3} j+\mu_{4} i j \in D$ mit $\mu_{t} \in F$.

\subsubsection{Verdopplung zur Oktavenalgebra}

Über das Verdopplungsverfahren (siehe Korollar 3.3.15) mit Unbestimmter $v$ und der Festlegung $N(v)=z$ konstruieren wir die Oktavenalgebra

$$
C=D \oplus D v .
$$

Für die Multiplikation, die Norm, die zugehörige Bilinearform und Konjugation in $C$ gilt

$$
\begin{aligned}
(a+b v)(c+d v) & =(a c-z \bar{d} b)+(d a+b \bar{c}) v, \\
N(a+b v) & =N(a)+z N(b), \\
\langle a+b v, c+d v\rangle & =\langle a, c\rangle+z\langle b, d\rangle \\
\overline{a+b v} & =\bar{a}-b v
\end{aligned}
$$

für $a, b, c, d \in D$. Als Standardbasis von $C$ über $F$ betrachten wir

$$
1, i, j, i j, v, i v, j v,(i j) v .
$$

Eine Multiplikationstafel für die Produkte zweier Basiselemente ungleich 1 ist in der Tabelle 5.1 aufgeführt. Insbesondere gilt für die Quadrate der vier neuen

\begin{tabular}{cccccccc}
\hline & \multicolumn{7}{c}{ 2. Faktor } \\
\cline { 2 - 8 } 1. Faktor & $i$ & $j$ & $i j$ & $v$ & $i v$ & $j v$ & $(i j) v$ \\
\hline$i$ & $-x$ & $i j$ & $-x j$ & $i v$ & $-x v$ & $-(i j) v$ & $x j v$ \\
$j$ & $-i j$ & $-y$ & $y i$ & $j v$ & $(i j) v$ & $-y v$ & $-y i v$ \\
$i j$ & $x j$ & $-y i$ & $-x y$ & $(i j) v$ & $-x j v$ & $y i v$ & $-x y v$ \\
$v$ & $-i v$ & $-j v$ & $-(i j) v$ & $-z$ & $z i$ & $z j$ & $z(i j)$ \\
$i v$ & $x v$ & $-(i j) v$ & $x j v$ & $-z i$ & $-x z$ & $-z(i j)$ & $x z j$ \\
$j v$ & $(i j) v$ & $y v$ & $-y i v$ & $-z j$ & $z(i j)$ & $-y z$ & $-y z i$ \\
$(i j) v$ & $-x j v$ & $y i v$ & $x y v$ & $-z(i j)$ & $-x z j$ & $y z i$ & $-x y z$ \\
\hline
\end{tabular}

Tabelle 5.1: Multiplikationstafel der Oktavenalgebra $C$

Basiselemente

$$
v^{2}=-z, \quad(i v)^{2}=-x z, \quad(j v)^{2}=-y z, \quad((i j) v)^{2}=-x y z .
$$


Schreiben wir $a+b v=\lambda=\left(\lambda_{1}, \lambda_{2}, \lambda_{3}, \lambda_{4}, \lambda_{5}, \lambda_{6}, \lambda_{7}, \lambda_{8}\right)$ und $c+d v=\mu=$ $\left(\mu_{1}, \mu_{2}, \mu_{3}, \mu_{4}, \mu_{5}, \mu_{6}, \mu_{7}, \mu_{8}\right) \in C$ bezüglich der ausgezeichneten Standardbasis mit Koeffizienten $\lambda_{t}, \mu_{t} \in F$, so gilt

$$
\begin{aligned}
N(\lambda)= & \lambda_{1}^{2}+\lambda_{2}^{2} x+\lambda_{3}^{2} y+\lambda_{4}^{2} x y+\lambda_{5}^{2} z+\lambda_{6}^{2} x z+\lambda_{7}^{2} y z+\lambda_{8}^{2} x y z \\
\langle\lambda, \mu\rangle= & 2\left(\lambda_{1} \mu_{1}+\lambda_{2} \mu_{2} x+\lambda_{3} \mu_{3} y+\lambda_{4} \mu_{4} x y\right. \\
& \left.\quad+\lambda_{5} \mu_{5} z+\lambda_{6} \mu_{6} x z+\lambda_{7} \mu_{7} y z+\lambda_{8} \mu_{8} x y z\right) \\
\bar{\lambda}= & \left(\lambda_{1},-\lambda_{2},-\lambda_{3},-\lambda_{4},-\lambda_{5},-\lambda_{6},-\lambda_{7},-\lambda_{8}\right) .
\end{aligned}
$$

Die Bilinearform hat die assoziierte Diagonalmatrix

$$
2 \operatorname{diag}(1, x, y, x y, z, x z, y z, x y z),
$$

die über $F=k(x, y, z)$ invertierbar ist. Folglich ist die Bilinearform auf $C$ in der Tat nichtausgeartet.

Für das Konjugierte und die Norm der Basiselemente in $C$ gilt insbesondere

$$
\begin{aligned}
& \overline{1}=1, \quad \bar{i}=-i, \quad \bar{j}=-j, \quad \overline{i j}=-i j, \\
& \bar{v}=-v, \quad \overline{i v}=-i v, \quad \overline{j v}=-j v, \quad \overline{(i j) v}=-(i j) v
\end{aligned}
$$

sowie

$$
\begin{aligned}
& N(1)=1, \quad N(i)=x, \quad N(j)=y, \quad N(i j)=x y, \\
& N(v)=z, \quad N(i v)=x z, \quad N(j v)=y z, \quad N((i j) v)=x y z .
\end{aligned}
$$

Somit ergeben sich als Inverse

$$
\begin{aligned}
1^{-1} & =1, & (i j)^{-1} & =-(x y)^{-1}(i j), \\
i^{-1} & =-x^{-1} i, & (i v)^{-1} & =-(x z)^{-1} i v, \\
j^{-1} & =-y^{-1} j, & (j v)^{-1} & =-(y z)^{-1} j v, \\
v^{-1} & =-z^{-1} v, & ((i j) v)^{-1} & =-(x y z)^{-1}(i j) v .
\end{aligned}
$$

Bemerkung 5.3.1. Die Norm $N$ ist anisotrop auf $D$ und auch auf $C$. Für jedes $\lambda \in C, \lambda \neq 0$ ist daher seine Norm $N(\lambda)$ in $F=k(x, y, z)$ invertierbar und auch $\lambda$ selbst ist in $C$ invertierbar mit Inversem $N(\lambda)^{-1} \bar{\lambda}$. Folglich ist $D$ eine Divisionsalgebra und $C$ eine Divisionsoktavenalgebra.

Beweis. Zuerst zeigen wir, dass im Polynomring $k[x, y]$ die Gleichung

$$
f_{1}^{2}+f_{2}^{2} x+f_{3}^{2} y+f_{4}^{2} x y=0
$$

nur die triviale Lösung $f_{1}=f_{2}=f_{3}=f_{4}=0$ hat. Dazu reicht es, neben der trivialen Lösung nur teilerfremde (nicht notwendig paarweise) $f_{1}, f_{2}, f_{3}, f_{4} \mathrm{zu}$ betrachten. Für eine solche nichttriviale Lösung der Gleichung, also

$$
-\left(f_{1}^{2}+f_{2}^{2} x\right)=\left(f_{3}^{2}+f_{4}^{2} x\right) y,
$$


folgt $f_{1}^{2}+f_{2}^{2} x \equiv 0$ in $k[x, y] /(y) \cong k[x]$ bzw. $-f_{1}^{2} \equiv f_{2}^{2} x(\bmod y)$. Da hiervon nicht die linke Seite in gerader, die rechte Seite jedoch in ungerader Potenz durch $x$ geteilt werden kann, folgt $f_{1} \equiv f_{2} \equiv 0(\bmod y)$. Damit können wir die gesamte Gleichung (5.1) durch $y$ kürzen und erhalten analog, dass auch die Polynome der rechten Seite durch $y$ teilbar sind. Insgesamt gilt also $f_{1} \equiv f_{2} \equiv f_{3} \equiv f_{4} \equiv 0(\bmod y)$ im Widerspruch zur betrachteten Teilerfremdheit.

Ebenso schließen wir im Polynomring $k[x, y, z]$ in drei Variablen. Für eine Lösung der Gleichung

$$
\left(f_{1}^{2}+f_{2}^{2} x+f_{3}^{2} y+f_{4}^{2} x y\right)+\left(f_{5}^{2}+f_{6}^{2} x+f_{7}^{2} y+f_{8}^{2} x y\right) z=0
$$

gilt $f_{1}^{2}+f_{2}^{2} x+f_{3}^{2} y+f_{4}^{2} x y \equiv 0$ in $k[x, y, z] /(z)$ und damit $f_{1} \equiv f_{2} \equiv f_{3} \equiv f_{4} \equiv 0$ $(\bmod z)$. Kürzen der Gleichung durch $z$ ergibt wiederum, dass $f_{t} \equiv 0(\bmod z)$ für alle $t=1, \ldots, 8$ gilt. Folglich existiert auch hier nur die triviale Lösung.

Sei nun $\lambda \in C$ ein Element mit verschwindender Norm $N(\lambda)=0$. Sei $g \neq 0$ der gemeinsame Hauptnenner der Koeffizienten $\lambda_{t}=\frac{f_{t}}{g}$ von $\lambda=\left(\lambda_{1}, \ldots, \lambda_{8}\right)$ für $t=1, \ldots, 8$. Dann ist auch der Zähler $g^{2} N(\lambda)$ gleich 0 , also

$$
\left(f_{1}^{2}+f_{2}^{2} x+f_{3}^{2} y+f_{4}^{2} x y\right)+\left(f_{5}^{2}+f_{6}^{2} x+f_{7}^{2} y+f_{8}^{2} x y\right) z=0,
$$

und aus den Vorüberlegungen folgt $\lambda=0$.

\subsubsection{Garbifizierung}

Sei $X=\left(\mathbb{P}_{k}^{3}, \mathcal{O}_{\mathbb{P}^{3}}\right)$ das projektive Schema von Dimension 3 über dem Körper $k$. In diesem Abschnitt soll gezeigt werden, dass die lokalfreie $\mathcal{O}_{\mathbb{P}^{3}}$-Modulgarbe

$$
\begin{aligned}
\mathcal{C}^{\prime \prime}= & \mathcal{O}_{\mathbb{P}^{3}} \oplus \mathcal{O}_{\mathbb{P}^{3}}(-1) i \oplus \mathcal{O}_{\mathbb{P}^{3}}(-1) j \oplus \mathcal{O}_{\mathbb{P}^{3}}(-1) i j \\
& \oplus \mathcal{O}_{\mathbb{P}^{3}}(-1) v \oplus \mathcal{O}_{\mathbb{P}^{3}}(-1) i v \oplus \mathcal{O}_{\mathbb{P}^{3}}(-1) j v \oplus \mathcal{O}_{\mathbb{P}^{3}}(-2)(i j) v
\end{aligned}
$$

eine Garbe von Oktavenalgebren und eine Garbe von Maximalordnungen auf $\operatorname{dem} \mathbb{P}^{3}$ ist, die $C$ fortsetzt, und in welchen Punkten $\mathcal{C}^{\prime \prime}$ gute bzw. schlechte Reduktion hat.

\section{Fortsetzung ins Affine}

Wir unterteilen den projektiven Raum in einen affinen Teil $\mathbb{A}^{3}$ und eine unendlich ferne Hyperebene $H_{\infty}$. Sei $\eta \in \mathbb{A}^{3} \subset \mathbb{P}^{3}$ der generische Punkt sowohl des affines Anteils als auch des gesamten projektiven Raums und sei $\xi_{\infty} \in H_{\infty}$ der generische Punkt der unendlich fernen Hyperebene $H_{\infty}$. Für die Funktionenkörper und die lokalen Ringe in den generischen Punkten gilt

$$
\begin{aligned}
k\left(\mathbb{P}^{3}\right) & =k\left(\mathbb{A}^{3}\right)=\mathcal{O}_{\mathbb{P}^{3}, \eta}=\mathcal{O}_{\mathbb{A}^{3}, \eta}=k(x, y, z) \quad \text { und } \\
\mathcal{O}_{\xi_{\infty}} & =\left\{\frac{f}{g} \in k(x, y, z) \mid \operatorname{deg} f \leq \operatorname{deg} g\right\},
\end{aligned}
$$


wobei mit deg der Totalgrad der Polynome bezeichnet sei.

Die obige Quaternionenalgebra $D$ und die Oktavenalgebra $C=D \oplus D v$ definieren $\mathcal{O}_{\mathbb{A}^{3}}$-Algebrengarben - im Falle von $C$ eine Garbe nichtassoziativer Algebren - auf dem affinen Teil:

$$
\begin{aligned}
& \mathcal{D}=\mathcal{O}_{\mathbb{A}^{3}} \oplus \mathcal{O}_{\mathbb{A}^{3}} i \oplus \mathcal{O}_{\mathbb{A}^{3}} j \oplus \mathcal{O}_{\mathbb{A}^{3}}(i j), \\
& \mathcal{C}=\mathcal{O}_{\mathbb{A}^{3}} \oplus \mathcal{O}_{\mathbb{A}^{3}} i \oplus \mathcal{O}_{\mathbb{A}^{3}} j \oplus \mathcal{O}_{\mathbb{A}^{3}}(i j) \oplus \mathcal{O}_{\mathbb{A}^{3}} v \oplus \mathcal{O}_{\mathbb{A}^{3}} i v \oplus \mathcal{O}_{\mathbb{A}^{3}} j v \oplus \mathcal{O}_{\mathbb{A}^{3}}(i j) v .
\end{aligned}
$$

Im generischen Punkt gilt dann offensichtlich $\mathcal{D}_{\eta}=D$ und $\mathcal{C}_{\eta}=C$. Die Multiplikation wird dabei aus $C$ übernommen - es gibt eine natürliche Einbettung von $\Gamma(U, \mathcal{C})$ in $C$ für alle offenen Mengen $U \subset \mathbb{A}^{3}$. Auch die Konjugation, Norm und Bilinearform vererben sich von $C$ auf die Mengen der Schnitte und definieren Garbenmorphismen $\mathcal{C} \rightarrow \mathcal{C}, \mathcal{C} \rightarrow \mathcal{O}_{\mathbb{A}^{3}}$ bzw. $\mathcal{C} \times \mathcal{C} \rightarrow \mathcal{O}_{\mathbb{A}^{3}}$.

Bemerkung 5.3.2. Für jeden Punkt $\mathfrak{p} \in \mathbb{A}^{3}$ (von beliebiger Kodimension) ist der Halm $\mathcal{C}_{\mathfrak{p}}$ eine Maximalordnung über $\mathcal{O}_{\mathfrak{p}}$ in $C$.

Folglich ist $\mathcal{C}$ eine Garbe von Maximalordnungen und damit als lokalfreie Garbe auch eine Garbe von Oktavenalgebren auf dem gesamten affinen Raum $\mathbb{A}^{3}$. Dabei hat $\mathcal{C}$ schlechte Reduktion in den Punkten der Verschwindungsmenge $\mathfrak{V}(x y z)$ - anschaulich sind das die Achsenkreuzebenen - und gute Reduktion auf dem offenen Komplement $\mathbb{A}^{3} \backslash \mathfrak{V}(x y z)$.

Beweis. Da $B=2 \operatorname{diag}(1, x, y, x y, z, x z, y z, x y z)$ die Matrix der Bilinearform auf $C$ und auch auf $\mathcal{C}$ ist, gilt in jedem Punkt $\mathfrak{p} \in \mathbb{A}^{3}$ für die lokale Algebra $\mathcal{C}_{\mathfrak{p}}$ und ihr duales Gitter:

$$
\begin{aligned}
\mathcal{C}_{\mathfrak{p}} & =\mathcal{O}_{\mathfrak{p}} \oplus \mathcal{O}_{\mathfrak{p}} i \oplus \mathcal{O}_{\mathfrak{p}} j \oplus \mathcal{O}_{\mathfrak{p}} i j \oplus \mathcal{O}_{\mathfrak{p}} v \oplus \mathcal{O}_{\mathfrak{p}} i v \oplus \mathcal{O}_{\mathfrak{p}} j v \oplus \mathcal{O}_{\mathfrak{p}}(i j) v \\
\mathcal{C}_{\mathfrak{p}}^{\#} & =\mathcal{O}_{\mathfrak{p}} \oplus \mathcal{O}_{\mathfrak{p}} \frac{i}{x} \oplus \mathcal{O}_{\mathfrak{p}} \frac{j}{y} \oplus \mathcal{O}_{\mathfrak{p}} \frac{i j}{x y} \oplus \mathcal{O}_{\mathfrak{p}} \frac{v}{z} \oplus \mathcal{O}_{\mathfrak{p}} \frac{i v}{x z} \oplus \mathcal{O}_{\mathfrak{p}} \frac{j v}{y z} \oplus \mathcal{O}_{\mathfrak{p}} \frac{(i j) v}{x y z}
\end{aligned}
$$

Für $\mathfrak{p} \notin \mathfrak{V}(x y z)$ ist $\operatorname{det} B=2^{8}(x y z)^{4}$ invertierbar in $\mathcal{C}_{\mathfrak{p}}^{\times}$und es gilt $\mathcal{C}_{\mathfrak{p}}^{\#}=\mathcal{C}_{\mathfrak{p}}$. Demnach ist $\mathcal{C}_{\mathfrak{p}}$ maximal in $C$ über $\mathcal{O}_{\mathfrak{p}}$. Außerdem folgt, dass auch die Quotientenalgebra $\mathcal{C}_{\mathfrak{p}} / \mathfrak{m}_{\mathfrak{p}} \mathcal{C}_{\mathfrak{p}}$ mit induzierter Norm eine Oktavenalgebra über dem Restklassenkörper $\mathcal{O}_{\mathfrak{p}} / \mathfrak{m}_{\mathfrak{p}}$ ist. Anders ausgedrückt hat $\mathcal{C}$ gute Reduktion in $\mathfrak{p} \in \mathbb{A}^{3} \backslash \mathfrak{V}(x y z)$. Hingegen ist für kein $\mathfrak{p} \in \mathfrak{V}(x y z)$ die Matrix der Bilinearform invertierbar über $\mathcal{O}_{\mathfrak{p}}$, daher hat $\mathcal{C}$ schlechte Reduktion auf $\mathfrak{V}(x y z)$.

Sei nun $\mathfrak{p} \in \mathbb{A}^{3}$ beliebig, also $\mathfrak{p}$ irgendein Primideal im Polynomring $k[x, y, z]$, und sei

$$
\lambda=\left(\frac{f_{1}}{g}, \frac{f_{2}}{g x}, \frac{f_{3}}{g y}, \frac{f_{4}}{g x y}, \frac{f_{5}}{g z}, \frac{f_{6}}{g x z}, \frac{f_{7}}{g y z}, \frac{f_{8}}{g x y z}\right) \in \mathcal{C}_{\mathfrak{p}}^{\#}
$$

ganz über $\mathcal{O}_{\mathfrak{p}}$; dabei seien $\frac{f_{t}}{g} \in \mathcal{O}_{\mathfrak{p}}=k[x, y, z]_{\mathfrak{p}}$ mit $f_{t}, g \in k[x, y, z]$ und $g \notin \mathfrak{p}$ für $t=1, \ldots, 8$. Aus $N(\lambda) \in \mathcal{O}_{\mathfrak{p}}$ und $g \notin \mathfrak{p}$ folgt, dass der Zähler

$$
(x y z) g^{2} N(\lambda)=\left(f_{1}^{2} x y+f_{2}^{2} y+f_{3}^{2} x+f_{4}^{2}\right) z+\left(f_{5}^{2} x y+f_{6}^{2} y+f_{7}^{2} x+f_{8}^{2}\right)
$$


im Ideal $(x y z) \mathcal{O}_{\mathfrak{p}}$ liegt.

Gilt $z \notin \mathfrak{p}$, so ist $z$ eine Einheit in $\mathcal{O}_{\mathfrak{p}}$, insbesondere gilt dann $z \mid f_{t}$ in $\mathcal{O}_{\mathfrak{p}}$ für $t=5,6,7,8$. Gilt hingegen $z \in \mathfrak{p}$, so ist $z$ nicht invertierbar in $\mathcal{O}_{\mathfrak{p}}$ und es liegt $(x y z) g^{2} N(\lambda)$ in $(z) \mathcal{O}_{\mathfrak{p}} \cap k[x, y, z]=(z) k[x, y, z]$. Insbesondere gilt dann

$$
f_{5}^{2} x y+f_{6}^{2} y+f_{7}^{2} x+f_{8}^{2} \equiv 0
$$

in $k[x, y, z] /(z) \cong k[x, y]$. Wie im Beweis zu Bemerkung 5.3.1, dass $N$ anisotrop ist, folgt hier $f_{5} \equiv f_{6} \equiv f_{7} \equiv f_{8} \equiv 0$ in $k[x, y, z] /(z)$. Mithin gilt auch in diesem Fall $z \mid f_{t}$ in $\mathcal{O}_{\mathfrak{p}}$ für $t=5,6,7,8$.

Ebenso folgt $x \mid f_{t}$ für $t=2,4,6,8$ und $y \mid f_{t}$ für $t=3,4,7,8$ in $\mathcal{O}_{\mathfrak{p}}$. Da $k[x, y, z]$ und $\mathcal{O}_{\mathfrak{p}}$ faktorielle Ringe sind und da $x, y, z$ paarweise teilerfremd sind, folgt daraus zusammen, dass stets alle Koeffizienten von $\lambda$ in $\mathcal{O}_{\mathfrak{p}}$ liegen. Somit gilt $\lambda \in \mathcal{C}_{\mathfrak{p}}$ und es folgt $\mathcal{C}_{\mathfrak{p}}^{(\#)}=\mathcal{C}_{\mathfrak{p}}$. Damit ist $\mathcal{C}_{\mathfrak{p}}$ eine Maximalordnung in $C$ über $\mathcal{O}_{\mathfrak{p}}$ für jedes Element $\mathfrak{p}$ der affinen Ebene $\mathbb{A}^{3}$.

\section{Fortsetzung ins Unendliche}

Über dem lokalen Ring $\mathcal{O}_{\infty}=\mathcal{O}_{\xi_{\infty}}$ der Strukturgarbe über dem generischen Punkt der unendlich fernen Hyperebene $H_{\infty}$ definieren wir den $\mathcal{O}_{\mathbb{P}^{3}}-$ Modul

$$
\mathcal{C}_{\infty}=\mathcal{O}_{\infty} \oplus \mathfrak{m}_{\infty} i \oplus \mathfrak{m}_{\infty} j \oplus \mathfrak{m}_{\infty} i j \oplus \mathfrak{m}_{\infty} v \oplus \mathfrak{m}_{\infty} i v \oplus \mathfrak{m}_{\infty} j v \oplus \mathfrak{m}_{\infty}^{2}(i j) v
$$

dabei bezeichne $\mathfrak{m}_{\infty}$ das maximale Ideal in $\mathcal{O}_{\infty}$. Auch $\mathcal{C}_{\infty}$ lässt sich in natürlicher Weise in die Algebra $C$ einbetten und erbt Multiplikation, Konjugation, Norm und Bilinearform von $C$. In der Tat ist $\mathcal{C}_{\infty}$ multiplikativ abgeschlossen, wie die Multiplikationstafel in Tabelle 5.2 für die letzten sieben direkten Summanden ohne $\mathcal{O}_{\infty}$ zeigt.

\begin{tabular}{cccccccc}
\hline & \multicolumn{7}{c}{2 Faktor } \\
\cline { 2 - 7 } 1. Faktor & $\mathfrak{m} i$ & $\mathfrak{m} j$ & $\mathfrak{m} i j$ & $\mathfrak{m} v$ & $\mathfrak{m} i v$ & $\mathfrak{m} j v$ & $\mathfrak{m}^{2}(i j) v$ \\
\hline $\mathfrak{m} i$ & $\mathfrak{m}$ & $\mathfrak{m}^{2} i j$ & $\mathfrak{m} j$ & $\mathfrak{m}^{2} i v$ & $\mathfrak{m} v$ & $\mathfrak{m}^{2}(i j) v$ & $\mathfrak{m}^{2} j v$ \\
$\mathfrak{m} j$ & $\mathfrak{m}^{2} i j$ & $\mathfrak{m}$ & $\mathfrak{m} i$ & $\mathfrak{m}^{2} j v$ & $\mathfrak{m}^{2}(i j) v$ & $\mathfrak{m} v$ & $\mathfrak{m}^{2} i v$ \\
$\mathfrak{m} i j$ & $\mathfrak{m} j$ & $\mathfrak{m} i$ & $\mathcal{O}_{\infty}$ & $\mathfrak{m}^{2}(i j) v$ & $\mathfrak{m} j v$ & $\mathfrak{m} i v$ & $\mathfrak{m} v$ \\
$\mathfrak{m} v$ & $\mathfrak{m}^{2} i v$ & $\mathfrak{m}^{2} j v$ & $\mathfrak{m}^{2}(i j) v$ & $\mathfrak{m}$ & $\mathfrak{m} i$ & $\mathfrak{m} j$ & $\mathfrak{m}^{2}(i j)$ \\
$\mathfrak{m} i v$ & $\mathfrak{m} v$ & $\mathfrak{m}^{2}(i j) v$ & $\mathfrak{m} j v$ & $\mathfrak{m} i$ & $\mathcal{O}_{\infty}$ & $\mathfrak{m}(i j)$ & $\mathfrak{m} j$ \\
$\mathfrak{m} j v$ & $\mathfrak{m}^{2}(i j) v$ & $\mathfrak{m} v$ & $\mathfrak{m} i v$ & $\mathfrak{m} j$ & $\mathfrak{m}(i j)$ & $\mathcal{O}_{\infty}$ & $\mathfrak{m} i$ \\
$\mathfrak{m}^{2}(i j) v$ & $\mathfrak{m}^{2} j v$ & $\mathfrak{m}^{2} i v$ & $\mathfrak{m} v$ & $\mathfrak{m}^{2}(i j)$ & $\mathfrak{m} j$ & $\mathfrak{m} i$ & $\mathfrak{m}$ \\
\hline
\end{tabular}

Tabelle 5.2: Multiplikationstafel der Oktavenalgebra $\mathcal{C}_{\infty}$

Da in dem lokalen Ring $\mathcal{O}_{\infty}$ alle gebrochenrationalen Polynomfunktionen $\frac{f}{g}$ mit $\operatorname{deg} f=\operatorname{deg} g$ invertierbar sind, enthält das maximale Ideal $\mathfrak{m}_{\infty}$ genau 
die Brüche $\frac{f}{g}$ mit $\operatorname{deg} f<\operatorname{deg} g$ und wird von jeder einzelnen rationalen Polynomfunktion von Gesamtgrad -1 erzeugt. Zum Beispiel gilt dann $\mathfrak{m}_{\infty}=$ $\frac{1}{x} \mathcal{O}_{\infty}$ und $\mathfrak{m}_{\infty}^{2}=\frac{1}{x^{2}} \mathcal{O}_{\infty}$ und wir erhalten mit

$$
1, \frac{i}{x}, \frac{j}{x}, \frac{i j}{x}, \frac{v}{x}, \frac{i v}{x}, \frac{j v}{x}, \frac{(i j) v}{x^{2}}
$$

eine Basis von $\mathcal{C}_{\infty}$ über $\mathcal{O}_{\infty}$. Bezüglich dieser Basis - die natürlich auch eine Basis von $C$ über $k(x, y, z)$ ist - hat die Bilinearform die assoziierte Diagonalmatrix

$$
B^{\prime}=2 \operatorname{diag}\left(1, \frac{1}{x}, \frac{y}{x^{2}}, \frac{y}{x}, \frac{z}{x^{2}}, \frac{z}{x}, \frac{y z}{x^{2}}, \frac{y z}{x^{3}}\right) .
$$

Da die Matrixeinträge sämtlich in $\mathcal{O}_{\infty}$ liegen, gilt tatsächlich wie zuvor behauptet $N(\lambda) \in \mathcal{O}_{\infty}$ und $\langle\lambda, \mu\rangle \in \mathcal{O}_{\infty}$ für alle $\lambda, \mu \in \mathcal{C}_{\infty}$. Da die Bilinearform nichtausgeartet ist, ist $\left(C_{\infty}, N_{\infty}\right)$ eine Oktavenalgebra über $\mathcal{O}_{\infty}$, die jedoch schlechte Reduktion modulo $\mathfrak{m}_{\infty}$ hat. Außerdem gilt für das duale Gitter von $\mathcal{C}_{\infty}$ in $C$ :

$$
\mathcal{C}_{\infty}^{\#}=\mathcal{O}_{\infty} \oplus \mathcal{O}_{\infty} i \oplus \mathcal{O}_{\infty} j \oplus \mathfrak{m}_{\infty} i j \oplus \mathcal{O}_{\infty} v \oplus \mathfrak{m}_{\infty} i v \oplus \mathfrak{m}_{\infty} j v \oplus \mathfrak{m}_{\infty}(i j) v
$$

Bemerkung 5.3.3. Es ist $\mathcal{C}_{\infty}$ eine Maximalordnung in $C$ über $\mathcal{O}_{\infty}$.

BEWEIS. Sei $\lambda=\lambda_{1}+\lambda_{2} i+\lambda_{3} j+\lambda_{4} i j+\lambda_{5} v+\lambda_{6} i v+\lambda_{7} j v+\lambda_{8}(i j) v \in \mathcal{C}_{\infty}^{(\#)}$ mit $\lambda_{t}=\frac{f_{t}}{g}$ und $f_{t}, g \in k[x, y, z]$ für $t=1, \ldots, 8$.

Dann gilt zum einen $\lambda \in \mathcal{C}_{\infty}^{\#}$ und damit $\operatorname{deg} f_{t} \leq \operatorname{deg} g$ für alle $t=1, \ldots, 8$ und $\operatorname{deg} f_{t}<\operatorname{deg} g$ für $t=4,6,7,8$. Es bezeichne $f_{t, d}$ die homogene Komponente von Grad $d$ von $f_{t}$ und es sei $d_{0}=\operatorname{deg} g$. Somit ergibt sich $f_{t, d}=0$ für $d>d_{0}$ für alle $t=1, \ldots, 8$ sowie $f_{t, d_{0}}=0$ für $t=4,6,7,8$.

Zum anderen ist

$$
N(\lambda)=\left(f_{1}^{2}+f_{2}^{2} x+f_{3}^{2} y+f_{4}^{2} x y+f_{5}^{2} z+f_{6}^{2} x z+f_{7}^{2} y z+f_{8}^{2} x y z\right) g^{-2} \in \mathcal{O}_{\infty}
$$

oder äquivalent

$$
\operatorname{deg}\left(f_{1}^{2}+f_{2}^{2} x+f_{3}^{2} y+f_{4}^{2} x y+f_{5}^{2} z+f_{6}^{2} x z+f_{7}^{2} y z+f_{8}^{2} x y z\right) \leq \operatorname{deg} g^{2} .
$$

Insbesondere verschwindet damit auch die homogene Komponente

$$
h_{2 d_{0}+1}=f_{2, d_{0}}^{2} x+f_{3, d_{0}}^{2} y+f_{5, d_{0}}^{2} z+f_{8, d_{0}-1}^{2} x y z
$$

von Grad $2 d_{0}+1$ von $g^{2} N(\lambda)$, dem Zähler von $N(\lambda)$. Es gilt also $h_{2 d_{0}+1}=0$ oder äquivalent

$$
N\left(\left(0, f_{2, d_{0}}, f_{3, d_{0}}, 0, f_{5, d_{0}}, 0,0, f_{8, d_{0}-1}\right)\right)=0 .
$$

Da die Norm auf $C$ anisotrop ist, folgt $f_{2, d_{0}}=f_{3, d_{0}}=f_{5, d_{0}}=f_{8, d_{0}-1}=0$. Letzteres bedeutet $\operatorname{deg} f_{t}<\operatorname{deg} g$ für $t=2,3,5$ und $\operatorname{deg} f_{8} \leq \operatorname{deg} g-2$. Somit gilt $\lambda_{t}=\frac{f_{t}}{g} \in \mathfrak{m}_{\infty}$ für $t=2,3,5$ und $\lambda_{8} \in \mathfrak{m}_{\infty}^{2}$. Zusammen mit der Voraussetzung $\lambda_{t} \in \mathfrak{m}_{\infty}$ für $t=4,6,7$ ergibt sich $\lambda \in \mathcal{C}_{\infty}$ und damit die Behauptung. 


\section{Gemeinsame Fortsetzung auf den gesamten $\mathbb{P}^{3}$}

Die beiden Fortsetzungen von $C$ auf $\mathbb{A}^{3}$ bzw. über den generischen Punkt $\xi_{\infty}$ der unendlich fernen Hyperebene

$$
\begin{aligned}
\mathcal{C} & =\mathcal{O}_{\mathbb{A}^{3}} \oplus \mathcal{O}_{\mathbb{A}^{3}} i \oplus \mathcal{O}_{\mathbb{A}^{3}} j \oplus \mathcal{O}_{\mathbb{A}^{3}}(i j) \oplus \mathcal{O}_{\mathbb{A}^{3}} v \oplus \mathcal{O}_{\mathbb{A}^{3}} i v \oplus \mathcal{O}_{\mathbb{A}^{3}} j v \oplus \mathcal{O}_{\mathbb{A}^{3}}(i j) v \\
\mathcal{C}_{\infty} & =\mathcal{O}_{\xi_{\infty}} \oplus \mathfrak{m}_{\infty} i \oplus \mathfrak{m}_{\infty} j \oplus \mathfrak{m}_{\infty} i j \oplus \mathfrak{m}_{\infty} v \oplus \mathfrak{m}_{\infty} i v \oplus \mathfrak{m}_{\infty} j v \oplus \mathfrak{m}_{\infty}^{2}(i j) v
\end{aligned}
$$

ergeben zusammen in natürlicher Weise eine Garbe $\mathcal{C}^{\prime}$ auf dem gesamten $\mathbb{P}^{3}$ : Für $U \subset \mathbb{P}^{3}$ offen sei

$$
\mathcal{C}^{\prime}(U)=\bigcap_{\substack{w \in U \\ w \in\left(\mathbb{P}^{3}\right)^{(1)}}} \mathcal{C}_{w} .
$$

Abhängig davon, ob $\xi_{\infty}$ in $U$ enthalten ist oder nicht - dabei ist $\xi_{\infty} \notin U$ äquivalent zu $U \subset A^{3}-$, gilt

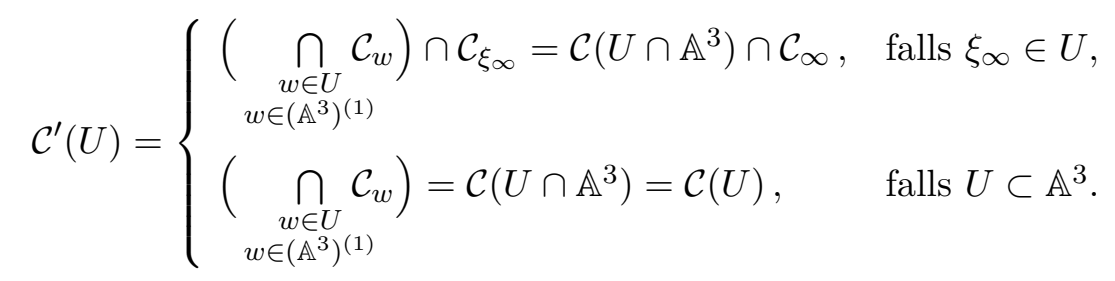

Insbesondere ist $\mathcal{C}^{\prime}$ eine Fortsetzung der beiden Garben auf $\mathbb{A}^{3}$ bzw. über $\xi_{\infty}$ :

$$
\left.\mathcal{C}^{\prime}\right|_{\mathbb{A}^{3}}=\mathcal{C} \quad \text { und } \quad \mathcal{C}_{\xi_{\infty}}^{\prime}=\mathcal{C}_{\infty}
$$

Da die Garbe $\mathcal{C}$ auf $\mathbb{A}^{3}$ und die Algebra $\mathcal{C}_{\infty}$ beide die Zerlegung in direkte Summanden von $C$ übernehmen, können wir für jede offene Menge $U \subset \mathbb{P}^{3}$, die $\xi_{\infty}$ enthält, den Durchschnitt $\mathcal{C}^{\prime}(U)=\mathcal{C}\left(U \cap \mathbb{A}^{3}\right) \cap \mathcal{C}_{\infty}$ komponentenweise berechnen. Es gilt:

$$
\begin{aligned}
\mathcal{C}^{\prime}(U)= & \mathcal{O}_{\mathbb{P}^{3}}(U) \oplus\left(\mathcal{O}_{\mathbb{A}^{3}}\left(U \cap \mathbb{A}^{3}\right) \cap \mathfrak{m}_{\infty}\right) i \\
& \oplus\left(\mathcal{O}_{\mathbb{A}^{3}}\left(U \cap \mathbb{A}^{3}\right) \cap \mathfrak{m}_{\infty}\right) j \oplus\left(\mathcal{O}_{\mathbb{A}^{3}}\left(U \cap \mathbb{A}^{3}\right) \cap \mathfrak{m}_{\infty}\right) i j \\
& \oplus\left(\mathcal{O}_{\mathbb{A}^{3}}\left(U \cap \mathbb{A}^{3}\right) \cap \mathfrak{m}_{\infty}\right) v \oplus\left(\mathcal{O}_{\mathbb{A}^{3}}\left(U \cap \mathbb{A}^{3}\right) \cap \mathfrak{m}_{\infty}\right) i v \\
& \oplus\left(\mathcal{O}_{\mathbb{A}^{3}}\left(U \cap \mathbb{A}^{3}\right) \cap \mathfrak{m}_{\infty}\right) j v \oplus\left(\mathcal{O}_{\mathbb{A}^{3}}\left(U \cap \mathbb{A}^{3}\right) \cap \mathfrak{m}_{\infty}^{2}\right)(i j) v
\end{aligned}
$$

\section{Isomorphie von $\mathcal{C}^{\prime}$ und $\mathcal{C}^{\prime \prime}$}

$\mathrm{Zu}$ einem Polynom $f \in k[x, y, z]$ mit $f \notin k$ bezeichne $\mathfrak{V}(f)$ die Verschwindungsmenge in $\mathbb{P}^{3}$ und $D_{+}(f)=\mathbb{P}^{3} \backslash \mathfrak{V}(f)$ deren offenes Komplement. - Genau genommen ist eigentlich die Verschwindungsmenge der Homogenisierung $\tilde{f}=f\left(\frac{x_{1}}{x_{0}}, \frac{y_{1}}{x_{0}}, \frac{z_{1}}{x_{0}}\right) x_{0}^{\operatorname{deg} f} \in S=k\left[x_{1}, y_{1}, z_{1}, x_{0}\right]$ in $\mathbb{P}^{3}=\operatorname{Proj}(S)$ zu betrachten. Dabei entsprechen die gewählte unendlich ferne Hyperebene $H_{\infty}$ und der affine Raum $\mathbb{A}^{3}$ der Verschwindungsmenge $\mathfrak{V}\left(x_{0}\right)$ und ihrem Komplement $D_{+}\left(x_{0}\right)$. 
Der affine Raum $\mathbb{A}^{3}$ bildet zusammen mit den drei offenen Mengen zu $x, y$ bzw. $z$ eine offene Überdeckung des projektiven Raumes:

$$
\mathbb{P}^{3}=\mathbb{A}^{3} \cup D_{+}(x) \cup D_{+}(y) \cup D_{+}(z) .
$$

Auf der zweiten offenen Menge dieser Überdeckung, $D_{+}(x)$, gilt

$$
\begin{aligned}
\mathcal{O}\left(D_{+}(x)\right) & =\left\{\frac{h}{x^{n}} \mid h \in k[x, y, z]_{x}, n \in \mathbb{N}_{0}, \operatorname{deg} h \leq n\right\}, \\
\mathcal{O}\left(D_{+}(x)\right) \cap \mathfrak{m}_{\infty} & =\left\{\frac{h}{x^{n}} \mid h \in k[x, y, z]_{x}, n \in \mathbb{N}_{0}, \operatorname{deg} h<n\right\} \\
& =\frac{1}{x} \mathcal{O}\left(D_{+}(x)\right), \\
\mathcal{O}\left(D_{+}(x)\right) \cap \mathfrak{m}_{\infty}^{2} & =\frac{1}{x^{2}} \mathcal{O}\left(D_{+}(x)\right) .
\end{aligned}
$$

Daher gilt für die Einschränkung der Garbe $\mathcal{C}^{\prime}$ auf $D_{+}(x)$ und analog auch für die Einschränkungen auf $D_{+}(y)$ und $D_{+}(z)$ :

$$
\begin{aligned}
\left.\mathcal{C}^{\prime}\right|_{\mathbb{A}^{3}} & =\mathcal{C}=\mathcal{O}_{\mathbb{A}^{3}} \oplus \mathcal{O} i \oplus \mathcal{O} j \oplus \mathcal{O}(i j) \oplus \mathcal{O} v \oplus \mathcal{O} i v \oplus \mathcal{O} j v \oplus \mathcal{O}(i j) v, \\
\left.\mathcal{C}^{\prime}\right|_{D_{+}(x)} & =\mathcal{O}_{D_{+}(x)} \oplus \mathcal{O} \frac{i}{x} \oplus \mathcal{O} \frac{j}{x} \oplus \mathcal{O} \frac{i j}{x} \oplus \mathcal{O} \frac{v}{x} \oplus \mathcal{O} \frac{i v}{x} \oplus \mathcal{O} \frac{j v}{x} \oplus \mathcal{O} \frac{(i j) v}{x^{2}}, \\
\left.\mathcal{C}^{\prime}\right|_{D_{+}(y)} & =\mathcal{O}_{D_{+}(y)} \oplus \mathcal{O} \frac{i}{y} \oplus \mathcal{O} \frac{j}{y} \oplus \mathcal{O} \frac{i j}{y} \oplus \mathcal{O} \frac{v}{y} \oplus \mathcal{O} \frac{i v}{y} \oplus \mathcal{O} \frac{j v}{y} \oplus \mathcal{O} \frac{(i j) v}{y^{2}}, \\
\left.\mathcal{C}^{\prime}\right|_{D_{+}(z)} & =\mathcal{O}_{D_{+}(z)} \oplus \mathcal{O} \frac{i}{z} \oplus \mathcal{O} \frac{j}{z} \oplus \mathcal{O} \frac{i j}{z} \oplus \mathcal{O} \frac{v}{z} \oplus \mathcal{O} \frac{i v}{z} \oplus \mathcal{O} \frac{j v}{z} \oplus \mathcal{O} \frac{(i j) v}{z^{2}} .
\end{aligned}
$$

Dabei ist etwa im zweiten direkten Summanden die Garbe auf dem $\mathbb{P}^{3}$, die sich als Verklebung von $\mathcal{O}_{\mathbb{A}^{3}}$ auf $\mathbb{A}^{3}$ und von $\mathcal{O}_{D_{+}(x)} \frac{1}{x}, \mathcal{O}_{D_{+}}(y) \frac{1}{y}$ und $\mathcal{O}_{D_{+}(z)} \frac{1}{z}$ auf $D_{+}(x), D_{+}(y)$ bzw. $D_{+}(z)$ ergibt, kanonisch isomorph zur getwisteten Garbe $\mathcal{O}_{\mathbb{P}^{3}}(-1)$. Die Garbe der letzten Summanden ist isomorph zu $\mathcal{O}_{\mathbb{P}^{3}}(-2)$, sodass wir insgesamt eine natürliche Isomorphie

$$
\begin{aligned}
\mathcal{C}^{\prime} \cong & \mathcal{O}_{\mathbb{P}^{3}} \oplus \mathcal{O}_{\mathbb{P}^{3}}(-1) i \oplus \mathcal{O}_{\mathbb{P}^{3}}(-1) j \oplus \mathcal{O}_{\mathbb{P}^{3}}(-1) i j \\
& \oplus \mathcal{O}_{\mathbb{P}^{3}}(-1) v \oplus \mathcal{O}_{\mathbb{P}^{3}}(-1) i v \oplus \mathcal{O}_{\mathbb{P}^{3}}(-1) j v \oplus \mathcal{O}_{\mathbb{P}^{3}}(-2)(i j) v
\end{aligned}
$$

erhalten.

\section{Maximalität von $\mathcal{C}^{\prime}$ auf $\operatorname{dem} \mathbb{P}^{3}$}

Bemerkung 5.3.4. Für jeden Punkt $\mathfrak{p} \in \mathbb{P}^{3}$ (von beliebiger Kodimension) ist der Halm $\mathcal{C}_{\mathfrak{p}}^{\prime}$ eine Maximalordnung über $\mathcal{O}_{\mathfrak{p}}$ in $C$.

Es ist $\mathcal{C}^{\prime}$ eine Garbe von Maximalordnungen und damit als lokalfreie Garbe auch eine Garbe von Oktavenalgebren auf dem gesamten Raum $\mathbb{P}^{3}$. Dabei hat $\mathcal{C}^{\prime}$ schlechte Reduktion in den Punkten der Verschwindungsmenge $\mathfrak{V}(x y z)$ sowie der unendlich fernen Hyperebene $H_{\infty}$ und gute Reduktion auf dem offenen Komplement $D_{+}(x y z) \backslash H_{\infty}=\mathbb{A}^{3} \backslash \mathfrak{V}(x y z)$. 
BEweIs. Zusammen mit der Hyperebene $\mathfrak{V}(x)$ bildet die offene Menge $D_{+}(x)$ eine weitere Zerlegung des projektiven Raumes in einen affinen Anteil und eine unendliche ferne Ebene. Es gilt:

$$
\begin{aligned}
\mathcal{O}\left(D_{+}(x)\right) & =k\left[x^{\prime}=\frac{1}{x}, y^{\prime}=\frac{y}{x}, z^{\prime}=\frac{z}{x}\right], \\
D_{+}(x) & =\operatorname{Spec} k\left[x^{\prime}, y^{\prime}, z^{\prime}\right] .
\end{aligned}
$$

Ein Punkt $\mathfrak{p} \in D_{+}(x)$ entspricht daher einem Primideal $\mathfrak{p} \subset k\left[x^{\prime}, y^{\prime}, z^{\prime}\right]$. In den neuen Koordinaten lässt sich die Einschränkung der Garbe $\left.\mathcal{C}^{\prime}\right|_{D_{+}(x)}$ auf $D_{+}(x)$ dann schreiben als

$$
\begin{aligned}
\mathcal{C}_{1} & =\mathcal{O} \oplus \mathcal{O} \frac{i}{x} \oplus \mathcal{O} \frac{j}{x} \oplus \mathcal{O} \frac{i j}{x} \oplus \mathcal{O} \frac{v}{x} \oplus \mathcal{O} \frac{i v}{x} \oplus \mathcal{O} \frac{j v}{x} \oplus \mathcal{O} \frac{(i j) v}{x^{2}} \\
& =\mathcal{O} \oplus \mathcal{O} x^{\prime} i \oplus \mathcal{O} x^{\prime} j \oplus \mathcal{O} x^{\prime} i j \oplus \mathcal{O} x^{\prime} v \oplus \mathcal{O} x^{\prime} i v \oplus \mathcal{O} x^{\prime} j v \oplus \mathcal{O}\left(x^{\prime}\right)^{2}(i j) v
\end{aligned}
$$

Die Matrix der Bilinearform lautet in dieser Basis

$$
\begin{aligned}
& 2 \operatorname{diag}\left(1, \frac{1}{x}, \frac{y}{x^{2}}, \frac{y}{x}, \frac{z}{x^{2}}, \frac{z}{x}, \frac{y z}{x^{2}}, \frac{y z}{x^{3}}\right) \\
= & 2 \operatorname{diag}\left(1, x^{\prime}, x^{\prime} y^{\prime}, y^{\prime}, x^{\prime} z^{\prime}, z^{\prime}, y^{\prime} z^{\prime}, x^{\prime} y^{\prime} z^{\prime}\right) .
\end{aligned}
$$

Diese Matrix sieht bis auf Permutation aus wie die Matrix der Bilinearform zur Garbe $\mathcal{C}$ auf der (standard-)affinen Ebene $\mathbb{A}^{3}$. Daher folgt ganz analog, dass auch hier $\mathcal{C}_{\mathfrak{p}}^{\prime(\#)}=\mathcal{C}_{\mathfrak{p}}^{\prime}$ für jeden Punkt $\mathfrak{p} \in D_{+}(x)$ gilt. Folglich ist $\left.\mathcal{C}^{\prime}\right|_{D_{+}(x)}$ eine Maximalordnung auf $D_{+}(x)$.

Insgesamt ist daher auch $\mathcal{C}^{\prime}$ eine Maximalordnung auf dem gesamten $\mathbb{P}^{3}$.

Zur Anschauung sei noch die Multiplikationstafel von $\mathcal{C}^{\prime}\left(D_{+}(x)\right)$ in Tabelle 5.3 angegeben.

\section{Alternative Konstruktion der Garbe}

Alternativ lässt sich die Garbe $\mathcal{C}^{\prime}$ auch ohne Hilfe von $\mathcal{C}_{\infty}$ durch Verkleben der vier Teilgarben $\mathcal{C}_{0}=\mathcal{C}, \mathcal{C}_{1}=\left.\mathcal{C}^{\prime}\right|_{D_{+}(x)}, \mathcal{C}_{2}=\left.\mathcal{C}^{\prime}\right|_{D_{+}(y)}$ und $\mathcal{C}_{3}=\left.\mathcal{C}^{\prime}\right|_{D_{+}(z)}$ auf den offenen Mengen $\mathbb{A}^{3}, D_{+}(x), D_{+}(y)$ und $D_{+}(z)$ konstruieren.

Die Multiplikation erben die Teilgarben von $C$, die Abgeschlossenheit für etwa $\mathcal{C}_{1}$ zeigt die Multiplikationstafel in Tabelle 5.3. Alle vier Garben $\mathcal{C}_{t}$ sind somit nichtassoziative $\mathcal{O}$-Algebrengarben.

Für eine offene Menge $V$ der Schnittmenge $\mathbb{A}^{3} \cap D_{+}(x)$ folgt aus $V \subset \mathbb{A}^{3}$, dass $x$ in $\mathcal{O}(V)$, und aus $V \subset D_{+}(x)$, dass $x^{-1}$ in $\mathcal{O}(V)$ liegt. Insgesamt ist $x \in \mathcal{O}(V)$ invertierbar und es gilt $\mathcal{O}(V)=\frac{1}{x} \mathcal{O}(V)$. Jeweils als Teilmengen von $C$ betrachtet gilt dann auch für die Algebren $\mathcal{C}_{1}(V)$ und $\mathcal{C}_{2}(V)$ Gleichheit. Folglich stimmen die beiden Garben $\mathcal{C}_{0}$ und $\mathcal{C}_{1}$ auf $\mathbb{A}^{3} \cap D_{+}(x)$ überein,

$$
\left.\mathcal{C}_{0}\right|_{\mathbb{A}^{3} \cap D_{+}(x)}=\left.\mathcal{C}_{1}\right|_{\mathbb{A}^{3} \cap D_{+}(x)},
$$




\begin{tabular}{cccccccc}
\hline & \multicolumn{7}{c}{ 2. Faktor } \\
\cline { 2 - 8 } 1. Faktor & $x^{\prime} i$ & $x^{\prime} j$ & $x^{\prime} i j$ & $x^{\prime} v$ & $x^{\prime} i v$ & $x^{\prime} j v$ & $x^{\prime 2}(i j) v$ \\
\hline$x^{\prime} i$ & $-x^{\prime}$ & $x^{\prime 2} i j$ & $-x^{\prime} j$ & $x^{\prime 2} i v$ & $-x^{\prime} v$ & $-x^{\prime 2}(i j) v$ & $x^{\prime 2} j v$ \\
$x^{\prime} j$ & $-x^{\prime 2} i j$ & $-y^{\prime} x^{\prime}$ & $y^{\prime} x^{\prime} i$ & $x^{\prime 2} j v$ & $x^{\prime 2}(i j) v$ & $-y^{\prime} x^{\prime} v$ & $-y^{\prime} x^{\prime 2} i v$ \\
$x^{\prime} i j$ & $x^{\prime} j$ & $-y^{\prime} x^{\prime} i$ & $-y^{\prime}$ & $x^{\prime 2}(i j) v$ & $-x^{\prime} j v$ & $y^{\prime} x^{\prime} i v$ & $-y^{\prime} x^{\prime} v$ \\
$x^{\prime} v$ & $-x^{\prime 2} i v$ & $-x^{\prime 2} j v$ & $-x^{\prime 2}(i j) v$ & $-z^{\prime} x^{\prime}$ & $z^{\prime} x^{\prime} i$ & $z^{\prime} x^{\prime} j$ & $z^{\prime} x^{\prime 2}(i j)$ \\
$x^{\prime} i v$ & $x^{\prime} v$ & $-x^{\prime 2}(i j) v$ & $x^{\prime} j v$ & $-z^{\prime} x^{\prime} i$ & $-z^{\prime}$ & $-z^{\prime} x^{\prime}(i j)$ & $z^{\prime} x^{\prime} j$ \\
$x^{\prime} j v$ & $x^{\prime 2}(i j) v$ & $y^{\prime} x^{\prime} v$ & $-y^{\prime} x^{\prime} i v$ & $-z^{\prime} x^{\prime} j$ & $z^{\prime} x^{\prime}(i j)$ & $-y^{\prime} z^{\prime}$ & $-y^{\prime} z^{\prime} x^{\prime} i$ \\
$x^{\prime 2}(i j) v$ & $-x^{\prime 2} j v$ & $y^{\prime} x^{\prime 2} i v$ & $y^{\prime} x^{\prime} v$ & $-z^{\prime} x^{\prime 2}(i j)$ & $-z^{\prime} x^{\prime} j$ & $y^{\prime} z^{\prime} x^{\prime} i$ & $-y^{\prime} z^{\prime} x^{\prime}$ \\
\hline
\end{tabular}

Tabelle 5.3: Multiplikationstafel der Oktavenalgebra $\mathcal{C}^{\prime}\left(D_{+}(x)\right)$

und können mit der Identität zu einer gemeinsamen Garbe auf der Vereinigung $\mathbb{A}^{3} \cup D_{+}(x)$ verklebt werden.

Ebenso gilt für eine offene Teilmenge $V \subset D_{+}(x) \cap D_{+}(y)$ : Aus $V \subset D_{+}(x)$ folgt $\frac{y}{x} \in \mathcal{O}(V)$ und aus $V \subset D_{+}(y)$ folgt $\frac{x}{y} \in \mathcal{O}(V)$. Somit gilt sowohl $\frac{1}{x} \mathcal{O}(V)=\frac{1}{y} \mathcal{O}(V)$ als auch

$$
\left.\mathcal{C}_{1}\right|_{D_{+}(x) \cap D_{+}(y)}=\left.\mathcal{C}_{2}\right|_{D_{+}(x) \cap D_{+}(y)} .
$$

Damit verkleben auch die beiden Garben $\mathcal{C}_{1}$ und $\mathcal{C}_{2}$ mittels der Identität auf $D_{+}(x) \cap D_{+}(y)$ zu einer gemeinsamen Garbe auf $D_{+}(x) \cup D_{+}(y)$.

Insgesamt verkleben die vier Garben $\mathcal{C}_{t}$ für $t=0,1,2,3$ so zu der oben angegebenen Garbe $\mathcal{C}^{\prime}$ auf $\mathbb{A}^{3} \cup D_{+}(x) \cup D_{+}(y) \cup D_{+}(z)=\mathbb{P}^{3}$. 



\section{Anhang A}

\section{Kommutative Algebra}

Sei $R$ ein kommutativer Ring mit Eins.

\section{A.1 Moduln und Algebren - Glossar}

Algebra Eine Algebra $A$ über einem Ring $R$ ist ein Ring $A$ mit Eins zusammen mit einem Ringhomomorphismus $\phi: R \rightarrow A$, dessen Bild im Zentrum von $A$ liegt. Die Algebra $A$ muss nicht notwendig kommutativ sein, die Abbildung $\phi$ muss nicht notwendig injektiv sein.

treu Ein Modul $M$ über einem Ring $R$ heißt treu, wenn sein Annullator trivial ist: $\operatorname{Ann}(M)=\{r \in R \mid r m=0 \forall m \in M\}=0$. Eine $R$-Algebra $A$ heißt treu, wenn sie als $R$-Modul treu ist. Dies ist genau dann der Fall, wenn die Strukturabbildung $R \rightarrow A, r \mapsto r 1_{A}$ injektiv ist.

endlich Eine Algebra über einem Ring heißt endlich, wenn sie als Modul endlich erzeugt ist. Eine endliche Algebra über einem Körper heißt entsprechend ihrer Eigenschaft als Vektorraum auch endlich-dimensional.

frei Ein Modul heißt frei, wenn er eine Basis, also ein linear unabhängiges Erzeugendensystem besitzt. Anders als bei Vektorräumen ist dies über beliebigen Ringen nicht zwingend der Fall.

endlich präsentiert Ein $R$-Modul $M$ heißt endlich präsentiert, wenn eine exakte Sequenz $N^{\prime} \rightarrow N \rightarrow M \rightarrow 0$ mit endlich erzeugten, freien $R$ Moduln $N, N^{\prime}$ existiert.

flach Ein $R$-Modul $M$ heißt flach, wenn der Funktor $N \mapsto M \otimes_{R} N$ auf der Kategorie der $R$-Moduln exakt ist. Da der Funktor stets rechtsexakt ist, ist dies genau dann der Fall, wenn die Injektivität von Morphismen beim Tensorieren mit $M$ erhalten bleibt. 
treuflach Ein $R$-Modul $M$ heißt treuflach, wenn die Sequenz

$$
\ldots \rightarrow N^{\prime} \rightarrow N \rightarrow N^{\prime \prime} \rightarrow \ldots
$$

von $R$-Moduln genau dann exakt ist, wenn die Sequenz

$$
\ldots \rightarrow M \otimes_{R} N^{\prime} \rightarrow M \otimes_{R} N \rightarrow M \otimes_{R} N^{\prime \prime} \rightarrow \ldots
$$

exakt ist. Es ist $M$ genau dann treuflach, wenn er flach ist und wenn $M \otimes_{R} N \neq 0$ für jeden $R$-Modul $N \neq 0$ gilt, und auch genau dann, wenn er flach ist und $\mathfrak{m} M \neq M$ für jedes maximale Ideal $\mathfrak{m}$ von $R$ gilt. Ein endlich erzeugter Modul ist genau dann treuflach, wenn er treu und flach ist.

projektiv Ein Modul heißt projektiv, wenn er direkter Summand eines freien Moduls ist. Projektive Moduln sind flach. Endlich erzeugte projektive Moduln sind endlich präsentiert.

treuprojektiv Ein Modul heißt treuprojektiv, wenn er projektiv und treuflach ist. Folglich ist ein endlich erzeugter Modul genau dann treuprojektiv, wenn er treu und projektiv ist.

einfach Eine Algebra über einem Körper heißt einfach, wenn sie keine nichttrivialen zweiseitigen Ideale besitzt.

zentral Eine Algebra über einem Ring heißt zentral, wenn ihr Zentrum nur das Bild des Grundringes ist. Manchmal wird zusätzlich gefordert, dass zentrale Algebren auch treu sein müssen; so definieren etwa Knus und Ojanguren in [KO74] Zentralität nur für treue Algebren.

\section{A.2 Nakayamas Lemma}

Die folgenden Aussagen werden zusammenfassend als Nakayamas Lemma bezeichnet.

Das Jacobson-Radikal $\operatorname{Rad}(S)$ eines nicht notwendig kommutativen Ringes $S$ ist der Durchschnitt all seiner maximalen Linksideale.

Satz A.2.1 (Nakayamas Lemma). Sei $S$ ein nicht notwendig kommutativer Ring mit Eins und sei $\mathfrak{a} \subset \operatorname{Rad}(S)$ ein zweiseitiges Ideal von S. Sei $M$ ein endlich erzeugter $S$-Linksmodul. Ist $\mathfrak{a} M=M$, so gilt $M=0$.

Lemma A.2.2. Sei $S$ ein nicht notwendig kommutativer Ring mit Eins und sei $\mathfrak{a} \subset \operatorname{Rad}(S)$ ein zweiseitiges Ideal von $S$. Sei $f: M \rightarrow N$ ein Homomorphismus von endlich erzeugten $S$-Linksmoduln. Ist id $\otimes f: S / \mathfrak{a} \otimes_{S} M \rightarrow S / \mathfrak{a} \otimes_{S} N$ eine surjektive Abbildung, so ist auch $f$ surjektiv.

Für lokale kommutative Ringe gelten auch die beiden folgenden Varianten: 
Lemma A.2.3. Seien $(R, \mathfrak{m})$ ein lokaler Ring, $M$ ein endlich erzeugter $R$-Modul und $N \subset M$ ein Untermodul. Ist $M=N+\mathfrak{m} M$, so gilt auch $M=N$.

Lemma A.2.4. Seien $(R, \mathfrak{m})$ ein lokaler Ring, $M$ ein endlich erzeugter $R$-Modul und $x_{1}, \ldots, x_{n} \in M$. Erzeugen die Restklassen $\overline{x_{1}}, \ldots, \overline{x_{n}}$ den Quotientenmodul $M / \mathfrak{m} M$, so erzeugen $x_{1}, \ldots, x_{n}$ den Modul $M$.

\section{A.3 Lokale Eigenschaften}

Lemma A.3.1. Sei $M$ ein R-Modul. Dann sind äquivalent:

a) $M=0$,

b) $M_{\mathfrak{p}}=0$ für jedes Primideal $\mathfrak{p} \subset R$,

c) $M_{\mathfrak{m}}=0$ für jedes maximale Ideal $\mathfrak{m} \subset R$.

BewEIs. Siehe [Str02, Lemma 14/1.14].

Die Eigenschaft $M=0$ lässt sich auch modulo maximaler Ideale testen:

Korollar A.3.2. Sei $M$ ein R-Modul. Dann gilt $M=0$ genau dann, wenn $M / \mathfrak{m} M=0$ für alle maximalen Ideale $\mathfrak{m} \subset R$ gilt.

BEWEIS. Nach dem vorherigen Lemma reicht es, diese Eigenschaft für einen lokalen Ring $(R, \mathfrak{m})$ zu zeigen. Sei $M / \mathfrak{m} M=0$. Da dann $\overline{0}=0+\mathfrak{m} M$ den Quotientenmodul $M / \mathfrak{m} M$ erzeugt, folgt nach Nakayamas Lemma, dass 0 jeden endlich erzeugten Untermodul von $M$ erzeugt. Damit muss $M$ selbst der Nullmodul sein.

Lemma A.3.3. Sei $\varphi: M \rightarrow N$ ein R-Modulhomomorphismus. Dann sind äquivalent:

a) $\varphi: M \rightarrow N$ ist injektiv (bzw. surjektiv),

b) $\varphi_{\mathfrak{p}}: M_{\mathfrak{p}} \rightarrow N_{\mathfrak{p}}$ ist injektiv für jedes Primideal $\mathfrak{p} \subset R$ (bzw. surjektiv),

c) $\varphi_{\mathfrak{m}}: M_{\mathfrak{m}} \rightarrow N_{\mathfrak{m}}$ ist injektiv für jedes maximale Ideal $\mathfrak{m} \subset R$ (bzw. surjektiv).

BeweIs. Siehe [Str02, Lemma 15/1.15].

Surjektivität und Bijektivität von Homomorphismen lassen sich ebenfalls in den Quotienten modulo maximaler Ideale testen. Für die Injektivität gilt nur eine Implikation:

Korollar A.3.4. Sei $\varphi: M \rightarrow N$ ein R-Modulhomomorphismus und sei $M$ endlich erzeugt. Dann gilt: 
1. Falls $\bar{\varphi}_{\mathfrak{m}}: M / \mathfrak{m} M \rightarrow N / \mathfrak{m} N$ für jedes maximale Ideal $\mathfrak{m} \subset R$ injektiv ist, so ist auch $\varphi: M \rightarrow N$ injektiv.

2. Es ist $\varphi: M \rightarrow N$ genau dann surjektiv, wenn $\bar{\varphi}_{\mathfrak{m}}: M / \mathfrak{m} M \rightarrow N / \mathfrak{m} N$ für jedes maximale Ideal $\mathfrak{m} \subset R$ surjektiv ist.

3. Es ist $\varphi: M \rightarrow N$ genau dann bijektiv, wenn $\bar{\varphi}_{\mathfrak{m}}: M / \mathfrak{m} M \rightarrow N / \mathfrak{m} N$ für jedes maximale Ideal $\mathfrak{m} \subset R$ bijektiv ist.

BEwEIs. Nach dem vorherigen Lemma reicht es, die Aussagen für einen lokalen $\operatorname{Ring}(R, \mathfrak{m})$ zu zeigen.

Ist $\bar{\varphi}=\bar{\varphi}_{\mathfrak{m}}: M / \mathfrak{m} M \rightarrow N / \mathfrak{m} N$ injektiv, so ist $\operatorname{ker} \bar{\varphi}=0$ und damit auch $(\operatorname{ker} \varphi) / \mathfrak{m} M \subset \operatorname{ker} \bar{\varphi}$ trivial. Nach dem letzten Korollar folgt $\operatorname{ker} \varphi=0$.

Ist $\bar{\varphi}$ surjektiv, so folgt aus Nakayamas Lemma, dass auch $\varphi$ selbst surjektiv ist. Die Umkehrung ist klar.

Ist $\varphi$ bijektiv mit Umkehrabbildung $\psi$, so ist die Reduktion $\bar{\psi}$ eine Umkehrabbildung zu $\bar{\varphi}$. Die Umkehrung folgt aus den ersten beiden Aussagen.

\section{A.4 Skalarerweiterung}

Satz A.4.1. Seien A eine beliebige und $S$ eine kommutative $R$-Algebra und seien $M$ und $N$ zwei A-Linksmoduln. Gilt

(a) $M$ ist endlich erzeugter und freier $A$-Modul oder

(b) $M$ ist endlich präsentierter A-Modul und $S$ ist flacher $R$-Modul, so ist der Morphismus

$$
\begin{aligned}
\varphi_{M}: \operatorname{Hom}_{A}(M, N) \otimes_{R} S & \longrightarrow \operatorname{Hom}_{A \otimes_{R} S}\left(M \otimes_{R} S, N \otimes_{R} S\right) \\
f \otimes s & \longmapsto f \otimes \ell_{s},
\end{aligned}
$$

mit der Linksmultiplikation $\ell_{s}: S \rightarrow S, x \mapsto s x$, bijektiv.

BeweIs. Siehe [Str02, Satz 17/1.17].

Korollar A.4.2. Seien $A$ und $S$ zwei kommutative $R$-Algebren und seien $P$ ein endlich erzeugter projektiver und $N$ ein beliebiger A-Modul. Dann ist der Morphismus

$$
\begin{aligned}
\varphi_{P}: \operatorname{Hom}_{A}(P, N) \otimes_{R} S & \longrightarrow \operatorname{Hom}_{A \otimes_{R} S}\left(P \otimes_{R} S, N \otimes_{R} S\right) \\
f \otimes s & \longmapsto f \otimes \ell_{s}
\end{aligned}
$$

bijektiv.

BeweIs. Da $P$ projektiv ist, existieren ein $R$-Modul $Q$ und eine Isomorphie $P \oplus Q \cong A^{n}$. Anwendung von (a) des letzten Satzes auf $M=A^{n}$ ergibt auch die gewünschte Isomorphie für den direkten Summanden $P$ von $A^{n}$. 


\section{A.5 Rang eines projektiven Moduls}

Anders als bei Vektorräumen über Körpern hat über einem Ring nicht jeder Modul eine Basis. Ist ein Modul endlich erzeugt und frei, besitzt also eine Basis, so haben alle Basen dieselbe endliche Anzahl Elemente; diese Anzahl bezeichnet man als Rang des Moduls. Allgemeiner kann man einen Rang oder eher eine Rangfunktion für endlich erzeugte, projektive Moduln definieren, indem man die Ränge der Lokalisierungen in den Primidealen betrachtet. Wir folgen den Ausführungen in Bourbakis Commutative Algebra, Ch. II Localization, $\$ 5.3$ Ranks of projective modules [Bou72].

Definition. Sei $P$ ein endlich erzeugter projektiver $R$-Modul. Für ein jedes Primideal $\mathfrak{p} \subset R$ sei der Rang von $P$ in $\mathfrak{p}$ der Rang des freien Moduls $P_{\mathfrak{p}}$ über dem lokalen Ring $R_{\mathfrak{p}}$ :

$$
\operatorname{rg}_{\mathfrak{p}} P=\operatorname{rg}_{R_{\mathfrak{p}}} P_{\mathfrak{p}} .
$$

Ein freier Modul von Rang $n$ ist auch in jedem Primideal von Rang $n$.

Definition. Ein projektiver Modul $P$ hat Rang $n$ (oder konstanten Rang $n$ ), falls $\operatorname{rg}_{\mathfrak{p}} P=n$ für jedes Primideal $\mathfrak{p} \subset R$ gilt.

Hauptsatz A.5.1 ([Bou72, II.5.2, aus Theorem 1]). Für jeden endlich erzeugten projektiven $R$-Modul $P$ ist die Funktion $\operatorname{Spec} R \rightarrow \mathbb{N}_{0}, \mathfrak{p} \mapsto \operatorname{rg}_{\mathfrak{p}} P$ lokal konstant.

Folglich ist über einem Ring mit zusammenhängendem Spektrum die Funktion konstant, insbesondere über jedem Integritätsbereich.

Hauptsatz A.5.2 ([Bou72, II.5.3, Theorem 2]). Sei P ein R-Modul. Dann sind äquivalent:

a) Es ist P projektiv von Rang n.

b) Es ist $P$ endlich erzeugt und für jedes Primideal $\mathfrak{p} \subset R$ ist $P_{\mathfrak{p}}$ ein freier $R_{\mathfrak{p}}$-Modul von Rang $n$.

c) Es ist $P$ endlich erzeugt und für jedes maximale Ideal $\mathfrak{m} \subset R$ ist $P_{\mathfrak{m}}$ ein freier $R_{\mathfrak{m}}$-Modul von Rang $n$.

d) Für jedes maximale Ideal $\mathfrak{m} \subset R$ existiert ein $f \in R \backslash \mathfrak{m}$ so, dass $P_{f}$ ein freier $R_{f}$-Modul von Rang $n$ ist.

Seien $E$ und $F$ endlich erzeugte projektive $R$-Moduln. Dann sind auch $E \times F$, $E \otimes_{R} F, \operatorname{Hom}_{R}(E, F), E^{*}=\operatorname{Hom}_{R}(E, R)$ und das äußere Produkt $\Lambda^{k} E$ (für alle $k \geq 0$ ) endlich erzeugte projektive $R$-Moduln. Für den Rang dieser Moduln in einem Primideal $\mathfrak{p} \subset R$ gilt

$$
\begin{aligned}
\operatorname{rg}_{\mathfrak{p}}(E \times F) & =\operatorname{rg}_{\mathfrak{p}}(E)+\operatorname{rg}_{\mathfrak{p}}(F), & \operatorname{rg}_{\mathfrak{p}}\left(\operatorname{Hom}_{R}(E, F)\right) & =\operatorname{rg}_{\mathfrak{p}}(E) \operatorname{rg}_{\mathfrak{p}}(F), \\
\operatorname{rg}_{\mathfrak{p}}(E \otimes F) & =\operatorname{rg}_{\mathfrak{p}}(E) \operatorname{rg}_{\mathfrak{p}}(F), & \operatorname{rg}_{\mathfrak{p}}\left(E^{*}\right) & =\operatorname{rg}_{\mathfrak{p}}(E)
\end{aligned}
$$


sowie

$$
\operatorname{rg}_{\mathfrak{p}} \Lambda^{k} E=\left(\begin{array}{c}
\operatorname{rg}_{\mathfrak{p}}(E) \\
k
\end{array}\right)
$$

Korollar. Sei $P$ ein endlich erzeugter projektiver $R$-Modul. Dann ist $P$ genau dann von Rang $n$, wenn $\Lambda^{n} P$ von Rang 1 ist.

\section{A.6 Zusammenhängende Ringe}

Wie wir im letzten Abschnitt gesehen haben, ist etwa der Rang eines projektiven Moduls lokal konstant auf den Zusammenhangskomponenten seines zugehörigen Primspektrums. Die topologischen Eigenschaften von $\operatorname{Spec} R$ haben also einen gewissen Einfluss auf algebraische Eigenschaften des Ringes $R$ oder eines Moduls über $R$. Von manchen Autoren wird der topologische Zusammenhangsbegriff direkt auf Ringe übertragen: Ein Ring $R$ heißt zusammenhängend, wenn sein Primspektrum Spec $R$ als topologischer Raum zusammenhängend ist.

Es lässt sich rein algebraisch beschreiben, wann ein Ring zusammenhängend ist, wie das folgende Lemma zeigt.

Sind $R_{1}$ und $R_{2}$ kommutative Ringe mit Eins, so ist ihr Produkt $R_{1} \times R_{2}$ in natürlicher Weise, nämlich mit komponentenweiser Addition und Multiplikation, ebenfalls ein kommutativer Ring mit Einselement $\left(1_{R_{1}}, 1_{R_{2}}\right)$.

Ein Element $x$ eines Ringes heißt idempotent, wenn $x^{2}=x$ gilt. Ist $x$ ein nichttriviales idempotentes Element, so ist dies auch $1-x$, denn dann gilt $(1-x)^{2}=1-2 x+x^{2}=1-2 x+x=1-x$. Außerdem gilt für Summe und Produkt dieser beiden Elemente dann $x+(1-x)=1$ und $x(1-x)=x-x^{2}=0$.

Lemma A.6.1. Sei $R$ ein kommutativer Ring mit Einselement 1. Dann sind die folgenden Eigenschaften äquivalent:

a) Es ist $R$ isomorph zu einem Produktring zweier Ringe $R_{1}, R_{2} \neq 0$.

b) Es gibt in $R$ zwei nichttriviale idempotente Elemente $e_{1}, e_{2} \neq 0,1$ mit $e_{1}+e_{2}=1$ und $e_{1} e_{2}=0$.

c) Das Spektrum $\operatorname{Spec} R$ ist als topologischer Raum nicht zusammenhängend.

Da die Äquivalenz dieser Bedingungen in der Literatur oftmals nur als Übungsaufgabe gestellt wird, wollen wir hier einen Beweis angeben.

Beweis. $(a \Rightarrow b)$ Ist $R=R_{1} \times R_{2}$ ein Produktring mit $R_{1}, R_{2} \neq 0$, so sind $e_{1}=\left(1_{R_{1}}, 0\right)$ und $e_{2}=\left(0,1_{R_{2}}\right)$ nichttriviale idempotente Elemente mit $e_{1}+e_{2}=1$ und $e_{1} e_{2}=0$.

$(b \Rightarrow a)$ Ist $e \in R$ ein idempotentes Element, so ist $R e \subset R$ ein Ring mit Einselement $e$. Ist dabei $e \neq 0$, so ist $R e$ nicht der Nullring. Seien 
nun $e_{1}, e_{2} \in R$ zwei nichttriviale idempotente Elemente mit $e_{1}+e_{2}=1$ und $e_{1} e_{2}=0$, dann definieren $R e_{1} \times R e_{2} \rightarrow R,\left(r_{1} e_{1}, r_{2} e_{2}\right) \mapsto r_{1} e_{1}+r_{2} e_{2}$ und $R \rightarrow R e_{1} \times R e_{2}, r \mapsto\left(r e_{1}, r e_{2}\right)$ zueinander inverse Ringhomomorphismen und somit eine nichttriviale Zerlegung $R \cong R e_{1} \times R e_{2}$.

$(b \Rightarrow c) \quad$ Seien $e_{1}$ und $e_{2}$ zwei nichttriviale Idempotente mit $e_{1}+e_{2}=1$ und $e_{1} e_{2}=0$. Da die einzige idempotente Einheit die 1 ist (ist $x^{2}=x$ und $x w=1$, so folgt $x=x \cdot 1=x^{2} w=x w=1$ ), sind die beiden abgeschlossenen Mengen

$$
V_{i}:=\mathfrak{V}\left(e_{i}\right)=\left\{\mathfrak{p} \in \operatorname{Spec} R \mid \mathfrak{p} \ni e_{i}\right\}
$$

für $i=1,2$ nichtleer. Außerdem sind sie disjunkt, denn kein Primideal enthält die Summe $e_{1}+e_{2}=1$. Jedes Primideal $\mathfrak{p}$ enthält mit $0=e_{1} e_{2}$ eines der beiden Elemente $e_{1}$ oder $e_{2}$. Damit ist Spec $R=V_{1} \cup V_{2}$ eine nichttriviale Zerlegung des Spektrums in zwei disjunkte abgeschlossene Mengen.

$(c \Rightarrow b) \quad$ Sei umgekehrt eine Zerlegung Spec $R=V_{1} \cup V_{2}$ in zwei nichtleere, disjunkte abgeschlossene Mengen $V_{1}, V_{2}$ gegeben. Dann gilt für die Verschwindungsideale $\mathfrak{I}\left(V_{i}\right)=\bigcap_{\mathfrak{p} \in V_{i}} \mathfrak{p}$ für $i=1,2$ :

$$
\begin{gathered}
\sqrt{(0)}=\mathfrak{I}(\operatorname{Spec} R)=\mathfrak{I}\left(V_{1} \cup V_{2}\right)=\mathfrak{I}\left(V_{1}\right) \cap \mathfrak{I}\left(V_{2}\right) \\
(1)=\mathfrak{I}(\emptyset)=\mathfrak{I}\left(V_{1} \cap V_{2}\right)=\sqrt{\mathfrak{I}\left(V_{1}\right)+\mathfrak{I}\left(V_{2}\right)} .
\end{gathered}
$$

Es gibt folglich zwei Elemente $x_{i} \in \mathfrak{I}\left(V_{i}\right)$ mit $1=x_{1}+x_{2}$. Da deren Produkt $x_{1} x_{2}$ im Nilradikal $\mathfrak{I}\left(V_{1}\right) \cap \mathfrak{I}\left(V_{2}\right)=\sqrt{(0)}$ liegt, gibt es ein $n \in \mathbb{N}$ mit $x_{1}^{n} x_{2}^{n}=0$. Für die Ideale $J_{i}=\left(x_{i}^{n}\right)$ und ihre Verschwindungsmengen gilt dann

$$
\mathfrak{V}\left(J_{1}\right) \cap \mathfrak{V}\left(J_{2}\right)=\mathfrak{V}\left(\left(x_{1}\right)\right) \cap \mathfrak{V}\left(\left(x_{2}\right)\right)=\mathfrak{V}\left(\left(x_{1}\right)+\left(x_{2}\right)\right)=\mathfrak{V}((1))=\emptyset
$$

und somit

$$
(1)=\mathfrak{I}(\emptyset)=\mathfrak{I}\left(\mathfrak{V}\left(J_{1}\right) \cap \mathfrak{V}\left(J_{2}\right)\right)=\sqrt{\sqrt{J_{1}}+\sqrt{J_{2}}}=\sqrt{J_{1}+J_{2}} .
$$

Es existieren daher zwei Elemente $e_{i} \in J_{i}$ mit $1=e_{1}+e_{2}$. Für ihr Produkt gilt diesmal $e_{1} e_{2} \in J_{1} J_{2}=(0)$, also $e_{1} e_{2}=0$. Damit gilt $0=e_{1} e_{2}=e_{1}\left(1-e_{1}\right)=$ $e_{1}-e_{1}^{2}$; ebenso folgt, dass auch $e_{2}$ idempotent ist.

Außerdem sind beide Elemente $e_{1}, e_{2} \neq 0,1$ : Da die $V_{i}$ nichtleer sind, sind $x_{i}$ und damit auch $x_{i}^{n} \in \mathfrak{I}\left(V_{i}\right)$ keine Einheiten und keines der Ideale $J_{i}$ enthält die 1.

\section{A.7 Going-up, Going-down}

In Lemma 2.4.7 verwenden wir, dass für eine endliche Überlagerung $p: Y \rightarrow X$ die Urbilder von Kodimension-1-Punkten ebenfalls von Kodimension 1 sind. Dies folgt aus den Going-up- und Going-down-Theoremen, die besagen, dass 
in einer ganzen Ringerweiterung Teilketten von Primidealen, die über einer längeren Primidealkette des Grundringes liegen, nach oben (going up) und nach unten (going down) fortgesetzt werden können. Daher wollen wir diese Sätze hier aufführen; wir folgen Atiyah und Macdonald [AM69, Ch. 5]:

Seien $A \subseteq B$ Integritätsbereiche und sei $B$ ganz über $A$.

Ist $\mathfrak{q}$ ein Primideal in $B$, so ist offensichtlich auch $\mathfrak{q} \cap A$ ein Primideal in $A$. Darüber hinaus gilt, dass $\mathfrak{q}$ genau dann ein maximales Ideal in $B$ ist, wenn auch $\mathfrak{q} \cap A$ maximal in $A$ ist. Sind $\mathfrak{q}^{\prime} \subseteq \mathfrak{q}$ zwei Primideale in $B$ mit $\mathfrak{q}^{\prime} \cap A=\mathfrak{q} \cap A$, dann gilt $\mathfrak{q}=\mathfrak{q}^{\prime}$. Beide Tatsachen folgen daraus, dass im Falle einer ganzen Erweiterung $B$ genau dann ein Körper ist, wenn $A$ ein Körper ist. (Siehe Proposition 5.7 und Korollare 5.8, 5.9 in [AM69].)

Außerdem liegt über jedem Primideal in $A$ ein Primideal in $B$ :

Hauptsatz A.7.1 ([AM69, Thm.5.10]). Seien $A \subseteq B$ Integritätsbereiche, dabei sei $B$ ganz über $A$, und sei $\mathfrak{p}$ ein Primideal in $A$. Dann existiert ein Primideal $\mathfrak{q}$ in $B$ mit $\mathfrak{q} \cap A=\mathfrak{p}$.

Durch Reduktion auf geeignete Quotienten erhalten wir daraus das Going-upTheorem:

Hauptsatz A.7.2 (Going-up, [AM69, Thm. 5.11]). Seien $A \subseteq B$ Integritätsbereiche, dabei sei $B$ ganz über $A$, und seien $\mathfrak{p}_{1} \subseteq \ldots \subseteq \mathfrak{p}_{n}$ eine Primidealkette in $A$ und $\mathfrak{q}_{1} \subseteq \ldots \subseteq \mathfrak{q}_{m}$ (mit $m<n$ ) eine Primidealkette in $B$ mit $\mathfrak{q}_{i} \cap A=\mathfrak{p}_{i}$ für $1 \leq i \leq m$. Dann kann die Primidealkette in $B$ zu einer Kette $\mathfrak{q}_{1} \subseteq \ldots \subseteq \mathfrak{q}_{n}$ mit $\mathfrak{q}_{i} \cap A=\mathfrak{p}_{i}$ für $1 \leq i \leq n$ erweitert werden.

Um eine Primidealkette nach unten fortsetzen zu können, benötigen wir noch die zusätzliche Voraussetzung, dass der Grundring ganzabgeschlossen ist:

Hauptsatz A.7.3 (Going-down, [AM69, Thm. 5.16]). Seien $A \subseteq B$ Integritätsbereiche, dabei sei $A$ ganzabgeschlossen und $B$ ganz über $A$, und seien $\mathfrak{p}_{1} \supseteq \ldots \supseteq \mathfrak{p}_{n}$ eine Primidealkette in $A$ und $\mathfrak{q}_{1} \supseteq \ldots \supseteq \mathfrak{q}_{m}$ (mit $m<n$ ) eine Primidealkette in $B$ mit $\mathfrak{q}_{i} \cap A=\mathfrak{p}_{i}$ für $1 \leq i \leq m$. Dann kann die Primidealkette in $B$ zu einer Kette $\mathfrak{q}_{1} \supseteq \ldots \supseteq \mathfrak{q}_{n}$ mit $\mathfrak{q}_{i} \cap A=\mathfrak{p}_{i}$ für $1 \leq i \leq n$ erweitert werden.

\section{A.8 Bewertungstheorie}

\section{A.8.1 Henselsches Lemma}

Satz A.8.1 (Henselsches Lemma). Sei $R$ ein vollständiger diskreter Bewertungsring mit Restklassenkörper $k$. Existiert zu einem primitiven Polynom $f \in R[T]$ eine Zerlegung seiner Reduktion $\bar{f} \in k[T]$ in teilerfremde Polynome $h_{1}, h_{2} \in k[T]$,

$$
\bar{f}=h_{1} h_{2},
$$


so existieren Polynome $g_{1}, g_{2} \in R[T]$ mit

$$
f=g_{1} g_{2}
$$

und

$$
\overline{g_{1}}=h_{1}, \quad \overline{g_{2}}=h_{2}, \quad \operatorname{grad} g_{1}=\operatorname{grad} h_{1} .
$$

BeweIs. Siehe [Ker07, Lemma 12.1] oder [Neu92, Kap. II, (4.6) Lemma].

\section{A.8.2 Fortsetzungen von Bewertungen}

Ist ein Körper vollständig bezüglich einer diskreten Bewertung, so lässt sich die Bewertung auf jede algebraische Erweiterung eindeutig fortsetzen. Ist ein Körper nicht vollständig bezüglich einer diskreten Bewertung, so existiert eine bis auf Isomorphie eindeutige Vervollständigung mit eindeutiger Fortsetzung der diskreten Bewertung. Diese kann man nutzen, um auch die Bewertung von nicht vollständigen diskret bewerteten Körpern auf endliche algebraische Erweiterungen fortzusetzen, wie wir im Folgenden zeigen:

Hauptsatz A.8.2 ([Neu92, Kap. II, (4.8) Theorem]). Sei K ein vollständiger diskret bewerteter Körper mit Bewertung v. Dann besitzt jede algebraische Erweiterung $L / K$ eine eindeutige Bewertungsfortsetzung $w$. Diese ist durch $w(\alpha)=\frac{1}{n} N_{L / K}(\alpha)$ gegeben, wenn $L / K$ endlichen Grad $n$ hat. In diesem Fall ist auch $L$ vollständig.

Wir betrachten nun eine endliche algebraische Erweiterung eines nicht notwendig vollständigen diskret bewerteten Körpers. Dabei beschränken wir uns in den kommenden Überlegungen auf separable Erweiterungen - in unseren Anwendungsfällen wird der Grundkörper Charakteristik 0 haben -, das Resultat gilt aber allgemeiner, wie der abschließend aufgeführte Satz aus Neukirch [Neu92] zeigt.

Es sei $K$ ein Körper mit diskreter Bewertung $x: K^{\times} \rightarrow \mathbb{Z}$ und Vervollständigung $K_{x}$. Ferner sei $K^{\prime}$ eine separable, endliche Erweiterung von $K$.

Dann existiert stets ein irreduzibles Polynom $f \in K[T]$ mit $K^{\prime} \cong K[T] /(f)$, und für das Tensorprodukt von $K_{x}$ und $K^{\prime}$ über $K$ gilt

$$
K_{x} \otimes_{K} K^{\prime}=K_{x} \otimes_{K} K[T] /(f) \cong K_{x}[T] /(f) .
$$

Dazu erhalten wir das kommutative Diagramm:

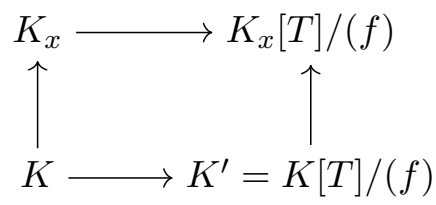


Im Polynomring $K_{x}[T]$ über der Vervollständigung $K_{x}$ zerlegen wir das Polynom $f$ als

$$
f=\prod_{i=1}^{r} p_{i}
$$

in seine irreduziblen Bestandteile $p_{i} \in K_{x}[T](i=1, \ldots, r)$. Da nach Voraussetzung $f$ über $K$ irreduzibel und $K^{\prime} / K$ separabel ist, hat $f$ auch über $K_{x}$ keine mehrfachen Nullstellen. Daher sind die Polynome $p_{i}$ paarweise teilerfremd und wir erhalten mit dem Chinesischen Restsatz die Isomorphie

$$
K_{x} \otimes_{K} K^{\prime} \cong K_{x}[T] /(f) \cong \prod_{i=1}^{r} K_{x}[T] /\left(p_{i}\right) .
$$

Es seien

$$
L_{i}=K_{x}[T] /\left(p_{i}\right)
$$

für $i=1, \ldots, r$ die Faktoren dieser Zerlegung. Dann ist jedes der $L_{i}$ eine endliche Körpererweiterung des vollständigen diskret bewerteten Körpers $K_{x}$ und folglich existiert jeweils eine eindeutig bestimmte Fortsetzung $y_{i}: L_{i}^{\times} \rightarrow \mathbb{Q}$ der zu $x$ gehörenden Bewertung von $K_{x}$. Es ist $L_{i}$ dann automatisch vollständig bezüglich $y_{i}$ und wir schreiben auch $L_{y_{i}}=L_{i}$.

Der Erweiterungsgrad von $L_{y_{i}} / K_{x}$ ist der Grad des Polynoms $p_{i}$ und es gilt

$$
\left[L_{y_{i}}: K_{x}\right]=\operatorname{grad} p_{i}=e_{i} f_{i},
$$

wobei $e_{i}$ der Verzweigungsindex der Bewertungsfortsetzung $y_{i}$ von $x$ und $f_{i}$ ihr Trägheitsgrad ist. Für den Verzweigungsindex $e_{i}$ gilt per definitionem

$$
\operatorname{im}\left(y_{i}: L_{y_{i}}^{\times} \rightarrow \mathbb{Q}\right)=\frac{1}{e_{i}} \mathbb{Z},
$$

und der Trägheitsgrad ist der Grad der Restklassenerweiterung,

$$
f_{i}=\left[k\left(y_{i}\right): k(x)\right],
$$

wobei $k(x)$ und $k\left(y_{i}\right)$ die Restklassenkörper der Bewertungsringe modulo maximaler Ideale der Bewertungen $x$ bzw. $y_{i}$ seien. Andererseits lassen sich Verzweigungsindex und Trägheitsgrad aber auch folgendermaßen bestimmen: Es sei $\overline{p_{i}} \in k(x)[T]$ die Restklasse von $p_{i}$ über dem Restklassenkörper $k(x)$. Die Zerlegung von $\overline{p_{i}}$ in $k(x)[T]$ ist dann von der Form

$$
\overline{p_{i}}=q_{i}^{e_{i}}
$$

mit einem geeigneten irreduziblen Polynom $q_{i} \in k(x)[T]$ und es gilt $k\left(y_{i}\right)=$ $k(x)[T] /\left(q_{i}\right)$ und somit

$$
f_{i}=\left[k\left(y_{i}\right): k(x)\right]=\operatorname{grad} q_{i}
$$


für $i=1, \ldots, r$.

Durch Einschränkung der Bewertungsfortsetzungen $y_{i}: L_{y_{i}}^{\times} \rightarrow \mathbb{Q}$ erhalten wir diskrete Bewertungen der ursprünglichen Körpererweiterung $K^{\prime} / K$, nämlich

$$
\left.y_{i}\right|_{K^{\prime}}: K^{\prime \times} \rightarrow \frac{1}{e_{i}} \mathbb{Z}
$$

für $i=1, \ldots, r$. Umgekehrt gilt auch, dass man jede Bewertungsfortsetzung von $x$ auf $K^{\prime}$ auf diese Art erhält. - Zusammenfassend sei hier der entsprechende Satz von Neukirch angegeben:

Satz A.8.3 ([Neu92, Kap. II, (8.2) Satz]). Sei K ein diskret bewerteter Körper mit Bewertung $x$. Die algebraische Erweiterung $K^{\prime} / K$ sei durch die Nullstelle $\alpha$ eines irreduziblen Polynoms $f \in K[T]$ erzeugt.

Dann entsprechen die Fortsetzungen $y_{1}, \ldots, y_{r}$ von $x$ auf $K^{\prime}$ umkehrbar eindeutig den irreduziblen Faktoren $p_{1}, \ldots, p_{r}$ in der Zerlegung $f=p_{1}^{m_{1}} \cdot \ldots \cdot p_{r}^{m_{r}}$ von $f$ über der Vervollständigung $K_{x}$.

Ist - wie bei uns - die Erweiterung $K^{\prime} / K$ und mithin auch das Polynom $f$ separabel, so gilt $m_{i}=1$ für $i=1, \ldots, r$.

Der Bewertungsring einer endlichen Körpererweiterung eines diskret bewerteten Körpers ergibt sich wie folgt aus dem Bewertungsring des Grundkörpers:

Lemma A.8.4 ([Neu92, Kap.II, Aufgabe 6]). Sei $K$ ein diskret bewerteter Körper mit diskreter Bewertung $v$ und Bewertungsring $R$. Sei $L / K$ eine endliche Körpererweiterung und $w$ eine Fortsetzung von $v$ auf L. Ist $\mathcal{O}$ der ganze Abschluss von $R$ in $L$, so ist die Lokalisierung $\mathcal{O}_{\mathfrak{p}}$ von $\mathcal{O}$ nach dem Primideal $\mathfrak{p}=\{\alpha \in \mathcal{O} \mid w(\alpha)>0\}$ der Bewertungsring von $w$.

\section{A.8.3 Verallgemeinerte Reihendarstellungen}

Ist $K$ ein diskret bewerteter Körper mit Uniformisierender $\pi$ und ist $S$ ein Vertretersystem des Restklassenkörpers, so besitzt jedes Element $x \in \hat{K}^{\times}$eine eindeutige Darstellung als konvergente Reihe $x=\sum_{i \geq v(x)} \alpha_{i} \pi^{i}$ mit $\alpha_{i} \in S$. Siehe etwa [Neu92, Kap. II, (4.4) Satz] oder [Ker07, Lemma 12.8 und 12.11].

In Abschnitt 4.4.1 zeigen wir, dass und wie sich eine diskrete Bewertung auf eine Kompositionsdivisionsalgebra über einem diskret bewerteten Körper fortsetzen lässt. In dieser erhalten wir dann eine analoge Darstellung der Elemente als konvergente Reihen:

Lemma A.8.5. Sei A eine nicht notwendig assoziative Divisionsalgebra über einem Körper $K$, die eine diskrete Exponentialbewertung $v: A \rightarrow \mathbb{Z}$ besitzt. Sei $\Delta=\{x \in A \mid v(x) \geq 0\}$ der Bewertungsring, $\mathfrak{m}=\{x \in A \mid v(x)>0\}$ sein maximales Ideal und $S \subset \Delta$ ein Vertretersystem für den Quotienten $\Delta / \mathfrak{m}$, das 
die 0 enthält. Für jedes $i \in \mathbb{Z}$ sei ein Element $\pi_{i} \in A^{\times}$mit $v\left(\pi_{i}\right)=i$ gegeben. Dann besitzt jedes Element $x \in A$ eine eindeutige Darstellung

$$
x=\sum_{i \geq v(x)} \alpha_{i} \pi_{i}
$$

mit $\alpha_{i} \in S$. Ist A vollständig, so konvergiert jede Reihe der obigen Form gegen ein $x \in A^{\times}$mit $v(x)=\min \left\{i \mid \alpha_{i} \neq 0\right\}$.

Wir folgen der Beweisführung von Kersten und modifizieren sie an entsprechenden Stellen:

BeweIs (nach [Ker07, Lemma 12.8]). Wir betrachten zunächst ein Element $x \in A^{\times}$mit positiver Bewertung $v(x) \geq 0$. Dann gilt $v\left(x \pi_{0}^{-1}\right)=v(x) \geq 0$, also ist $x \pi_{0}^{-1} \in \Delta$. Daher existiert ein eindeutiges $\alpha_{0} \in S$ mit

$$
x \pi_{0}^{-1} \equiv \alpha_{0} \quad \bmod \mathfrak{m} .
$$

Setze $s_{0}=\alpha_{0} \pi_{0}$, dann gilt $v\left(x-s_{0}\right) \geq 1$. Wir schließen per Induktion weiter: Sei eine Darstellung

$$
s_{n-1}=\alpha_{0} \pi_{0}+\alpha_{1} \pi_{1}+\ldots+\alpha_{n-1} \pi_{n-1}
$$

mit $\alpha_{i} \in S$ und

$$
v\left(x-s_{n-1}\right) \geq n
$$

gegeben. Dann gilt $\left(x-s_{n-1}\right) \pi_{n}^{-1} \in \Delta$ und es existiert ein eindeutiges $\alpha_{n} \in S$ mit

$$
\left(x-s_{n-1}\right) \pi_{n}^{-1} \equiv \alpha_{n} \quad \bmod \mathfrak{m} .
$$

Für dieses gilt

$$
v\left(\left(x-s_{n-1}\right) \pi_{n}^{-1}-\alpha_{n}\right)>0 .
$$

Setze

$$
s_{n}:=s_{n-1}+\alpha_{n} \pi_{n}=\alpha_{0} \pi_{0}+\alpha_{1} \pi_{1}+\ldots+\alpha_{n} \pi_{n},
$$

dann gilt

$$
x-s_{n}=x-s_{n-1}-\alpha_{n} \pi_{n}=\left(\left(x-s_{n-1}\right) \pi_{n}^{-1}-\alpha_{n}\right) \pi_{n}
$$

und damit

$$
v\left(x-s_{n}\right)=v\left(\left(x-s_{n-1}\right) \pi_{n}^{-1}-\alpha_{n}\right)+v\left(\pi_{n}\right) \geq n+1 .
$$

Sei nun $x \in A^{\times}$ein Element mit beliebiger Bewertung $n_{0}:=v(x) \in \mathbb{Z}$. Dann gilt $v\left(x \pi_{n_{0}}^{-1}\right)=0$, also $x \pi_{n_{0}}^{-1} \in \Delta$. Daher existiert ein eindeutiges $\alpha_{n_{0}} \in S$ mit

$$
x \pi_{n_{0}}^{-1} \equiv \alpha_{n_{0}} \quad \bmod \mathfrak{m} .
$$


Setze $s_{n_{0}}=\alpha_{n_{0}} \pi_{n_{0}}$, dann gilt

$$
v\left(x-s_{n_{0}}\right)=v\left(x \pi_{n_{0}}^{-1}-\alpha_{n_{0}}\right)+v\left(\pi_{n_{0}}\right) \geq 1+n_{0} .
$$

Weiter geht es wie zuvor.

Da für die Folge $\left(s_{n}\right)_{n \geq n_{0}}$ mit $s_{n}=\alpha_{n_{0}} \pi_{n_{0}}+\ldots+\alpha_{n} \pi_{n}$ dann stets $v\left(x-s_{n}\right) \geq n$ gilt, konvergiert die Folge gegen $x$. Also gilt am Ende tatsächlich

$$
x=\sum_{n \geq v(x)} \alpha_{n} \pi_{n} .
$$

Die Eindeutigkeit der Darstellung ist offensichtlich.

\section{A.9 Krullringe}

In diesem Abschnitt werden kurz die Definition und einige erste Eigenschaften von Krullringen zusammengefasst, so, wie sie in Bourbakis Commutative Algebra, Ch. VII Divisors [Bou72] eingeführt werden.

Definition ([Bou72, VII.1.3]). Ein Integritätsbereich $R$ heißt Krullring, falls eine Familie diskreter Bewertungen $\left(v_{i}\right)_{i \in I}$ des Quotientenkörpers $K=\operatorname{Quot}(R)$ so existiert, dass

1. der Ring $R$ der Durchschnitt der zugehörigen Bewertungsringe $\mathcal{O}_{v_{i}}$ ist, also $R=\bigcap_{i \in I} \mathcal{O}_{v_{i}}$, und dass

2. für jedes $x \in K^{\times}$die Menge $\left\{i \in I \mid v_{i}(x) \neq 0\right\}$ endlich ist.

Es reicht dabei offensichtlich, die letzte Bedingung für $x \in R \backslash\{0\}$ zu testen.

Beispiele für Krullringe sind offensichtlich jeder diskrete Bewertungsring, aber auch jeder Hauptidealring. Außerdem sind für jeden Krullring $R$ auch der Polynomring $R[X]$ und der Ring $R[[X]]$ der formalen Potenzreihen Krullringe.

Definition ([Bou72, V.1.4]). Ein Integritätsbereich $R$ mit Quotientenkörper $K$ heißt vollständig ganzabgeschlossen, wenn jedes $x \in K$, dessen sämtliche Potenzen $x^{n}$ (für $n \geq 0$ ) in einem endlich erzeugten $R$-Untermodul von $K$ enthalten sind, bereits in $R$ liegt.

Dabei gilt, dass alle $x^{n}$ genau dann in einem endlich erzeugten $R$-Untermodul von $K$ enthalten sind, wenn es ein $d \in R, d \neq 0$ mit $d x^{n} \in R$ für alle $n \geq 0$ gibt.

Jeder vollständig ganzabgeschlossene Ring ist auch ganzabgeschlossen. Umgekehrt ist jeder ganzabgeschlossene noethersche Ring auch vollständig ganzabgeschlossen; für allgemeine ganzabgeschlossene Ringe gilt dies jedoch nicht (siehe Bourbakis Bemerkungen zur Definition).

Krullringe sind ganzabgeschlossen und vollständig ganzabgeschlossen: 
Hauptsatz A.9.1 ([Bou72, VII.1.3, Thm.2]). Ein Integritätsbereich $R$ ist genau dann ein Krullring, wenn gilt:

1. Es ist $R$ vollständig ganzabgeschlossen.

2. Jede nichtleere Familie divisorialer ganzer Ideale von $R$ enthält ein maximales Element (bzgl. der Inklusionsrelation).

Korollar A.9.2 ([Bou72, VII.1.3, Corollary]). Ein noetherscher Ring ist genau dann ein Krullring, wenn er ganzabgeschlossen ist.

Nicht jeder Krullring muss noethersch sein: Sei $k$ ein Körper. Dann ist der Polynomring $k\left[X_{i}\right]_{i \in \mathbb{N}}$ in unendlich vielen Unbestimmten ein nicht noetherscher Krullring.

Hauptsatz A.9.3 ([Bou72, VII.1.6, Thm.4]). Sei $R$ ein Integritätsbereich und $P$ die Menge seiner Primideale von Höhe 1 . Dann ist $R$ genau dann ein Krullring, wenn gilt:

1. Für jedes $\mathfrak{p} \in P$ ist $R_{\mathfrak{p}}$ ein diskreter Bewertungsring.

2. Es gilt $R=\bigcap_{\mathfrak{p} \in P} R_{\mathfrak{p}}$.

3. Für jedes $x \in R, x \neq 0$ gibt es nur endlich viele $\mathfrak{p} \in P$ mit $x \in \mathfrak{p}$.

Außerdem sind die Bewertungen, die zu den $R_{\mathfrak{p}}$ für $\mathfrak{p} \in P$ korrespondieren, die wesentlichen Bewertungen (essential valuations) von $R$. 


\section{Anhang B}

\section{Garben und Schemata}

\section{B.1 Modulgarben und Schemata}

Definition. Ein Schema $\left(X, \mathcal{O}_{X}\right)$ heißt ganz, wenn für jede offene Menge $U \subset X$ der Ring $\mathcal{O}_{X}(U)$ ein Integritätsbereich ist.

Definition. Ein Schema heißt lokal-noethersch, wenn es eine Überdeckung durch offene affine Teilmengen $\operatorname{Spec}\left(R_{i}\right)$ mit noetherschen Ringen $R_{i}$ gibt. Ein Schema heißt noethersch, wenn es lokal-noethersch und quasikompakt ist.

Satz B.1.1 ([Har77, Ch. II, Proposition 3.2]). Ein Schema ist genau dann lokal-noethersch, wenn jede offene affine Teilmenge $\operatorname{Spec}(R)$ das Spektrum eines noetherschen Ringes $R$ ist.

Definition. Ein Schema $\left(X, \mathcal{O}_{X}\right)$ heißt normal, wenn für jeden Punkt $x \in X$ der lokale Ring $\mathcal{O}_{X, x}$ ein ganzabgeschlossener Integritätsbereich ist.

Satz B.1.2 ([AM69, Proposition 5.13]). Sei R ein Integritätsbereich. Dann sind äquivalent:

a) Es ist $R$ ganzabgeschlossen.

b) Für jedes Primideal $\mathfrak{p} \subset R$ ist $R_{\mathfrak{p}}$ ganzabgeschlossen.

c) Für jedes maximale Ideal $\mathfrak{m} \subset R$ ist $R_{\mathfrak{m}}$ ganzabgeschlossen.

Korollar B.1.3. Ist $\left(X, \mathcal{O}_{X}\right)$ ein normales Schema, so ist für jede zusammenhängende offene affine Menge $U=\operatorname{Spec}(R)$ der Ring $\mathcal{O}_{X}(U)$ ganzabgeschlossen.

Definition. Ein $\mathcal{O}_{X}$-Modul $\mathcal{F}$ heißt quasikohärent, wenn $X$ eine offene affine Überdeckung $\left(U_{i}=\operatorname{Spec}\left(R_{i}\right)\right)$ besitzt, für die jede Einschränkung $\left.\mathcal{F}\right|_{U_{i}}$ isomorph zu der assoziierten Garbe $\tilde{M}_{i}$ eines $R_{i}$-Modul $M_{i}$ ist.

Ein $\mathcal{O}_{X}$-Modul $\mathcal{F}$ heißt kohärent, wenn zusätzlich jedes $M_{i}$ als endlich erzeugter $R_{i}$-Modul gewählt werden kann. 
Satz B.1.4 ([Har77, Ch. II, Proposition 5.4]). Es gibt genau dann eine offene affine Überdeckung, die die Quasikohärenzbedingung erfüllt, wenn jede offene affine Überdeckung sie erfüllt. Ist $X$ ein noethersches Schema, so gilt die entsprechende Aussage auch für die Kohärenzbedingung.

Definition. Ein $\mathcal{O}_{X}$-Modul $\mathcal{F}$ heißt frei, wenn $\mathcal{F}$ zu einer direkten Summe von Kopien von $\mathcal{O}_{X}$ isomorph ist.

Ein $\mathcal{O}_{X}$-Modul $\mathcal{F}$ heißt lokalfrei, wenn es eine solche Überdeckung durch offene Teilmengen $U_{i}$ gibt, dass jedes $\left.\mathcal{F}\right|_{U_{i}}$ ein freier $\left.\mathcal{O}_{X}\right|_{U_{i}}$-Modul ist.

Satz B.1.5 ([Har77, aus Ch. II, Ex. 5.7]). Sei $\left(X, \mathcal{O}_{X}\right)$ ein noethersches Schema und sei $\mathcal{F}$ ein kohärenter $\mathcal{O}_{X}$-Modul. Dann gilt:

Ist der Halm $\mathcal{F}_{x}$ in dem Punkt $x \in X$ ein freier $\mathcal{O}_{X}$-Modul, so existiert eine solche Umgebung $U$ von $x$, dass $\left.\mathcal{F}\right|_{U}$ ein freier $\left.\mathcal{O}_{X}\right|_{U}$-Modul ist. Es ist $\mathcal{F}$ genau dann lokalfrei, wenn für alle $x \in X$ der Halm $\mathcal{F}_{x}$ ein freier $\mathcal{O}_{X, x}$-Modul ist.

Definition. Ein $R$-Modul $M$ heißt torsionsfrei, wenn $r m \neq 0$ für alle $r \in$ $R \backslash\{0\}$ und $m \in M \backslash\{0\}$ gilt.

Ein kohärenter $\mathcal{O}_{X}$-Modul $\mathcal{F}$ auf einem Schema $\left(X, \mathcal{O}_{X}\right)$ heißt torsionsfrei, wenn für jede offene affine Menge $U \subseteq X$ der $\mathcal{O}_{X}(U)$-Modul $\mathcal{F}(U)$ torsionsfrei ist.

Auch hierfür reicht es, die Bedingung für die Mengen einer beliebigen offenen affinen Überdeckung zu testen.

Satz B.1.6 ([HL97, Bemerkung nach Def.1.1.4]). Sei $\left(X, \mathcal{O}_{X}\right)$ ein ganzes, noethersches Schema. Dann ist ein kohärenter $\mathcal{O}_{X}$-Modul $\mathcal{F}$ genau dann torsionsfrei, wenn $\mathcal{F}_{x}$ für jedes $x \in X$ torsionsfrei ist.

Satz B.1.7 ([Fri98, Def. 2.19]). Sei $\left(X, \mathcal{O}_{X}\right)$ ein reguläres Schema. Dann ist ein kohärenter $\mathcal{O}_{X}$-Modul $\mathcal{F}$ genau dann torsionsfrei, wenn $\mathcal{F}_{x}$ für jedes $x \in X$ torsionsfrei ist.

\section{B.2 Garben von Ordnungen}

Allgemeiner als die lokalfreien Garben von Azumaya-Algebren werden auch Garben von Maximalordnungen betrachtet. Insbesondere da häufig die Brauergruppe des betrachteten Schemas trivial sein kann, etwa für Kurven über algebraisch abgeschlossenen Körpern (Satz von Tsen).

Wir geben hier drei in der Literatur vertretene Varianten zur Definition von Ordnungen an. In allen Fällen gilt: Eine Maximalordnung ist eine Ordnung, die in keiner anderen Ordnung echt enthalten ist.

Milne gibt die folgende Definition: 
Definition ([Mil80, S.150/151]). Sei $X$ ein ganzes, lokal-noethersches Schema mit Funktionenkörper $k(X)=K$ und sei $A_{K}$ eine endliche $K$-Algebra. Dann ist eine $\mathcal{O}_{X}$-Ordnung in $A_{K}$ eine kohärente $\mathcal{O}_{X}$-Algebra $\mathcal{A}$, die lokal in der Zariski-Topologie auf $X$ eine Ordnung ist.

In ihrer nicht veröffentlichten Arbeit „Stable orders over surfaces“ [AdJ] geben Artin und de Jong die folgende Definition:

Definition ([AdJ, 1.8]). Sei $X$ ein ganzes, noethersches Schema mit Funktionenkörper $k(X)=K$, und sei $A_{K}$ eine endliche $K$-Algebra. Eine $\mathcal{O}_{X}$-Ordnung in $A_{K}$ ist eine torsionsfreie kohärente $\mathcal{O}_{X}$-Algebra $\mathcal{A}$ mit genereller Faser $A_{K}$.

Letzteres bedeutet, dass ein Isomorphismus $\mathcal{A} \otimes_{\mathcal{O}_{X}} K \cong A_{K}$ existiert und fest gewählt wird.

Außerdem zeigen sie, dass Ordnungen existieren und dass für eine Maximalordnung jede lokale Algebra ebenfalls eine Maximalordnung ist:

Satz B.2.1 ([AdJ, 1.8.1 Prop.]). Sei $X$ ein ganzes, noethersches Schema mit Funktionenkörper $K$ und sei $\mathcal{A}$ eine Ordnung in einer endlichen $K$-Algebra $A_{K}$. Dann gilt:

1. Sei $j: U^{\prime} \rightarrow X$ die Einbettung einer nichtleeren, offenen Menge oder von $U^{\prime}=\operatorname{Spec} K$. Sei $\mathcal{B}$ eine endliche $\mathcal{O}_{X}$-Algebra mit $K \otimes \mathcal{B} \subset A_{K}$ und sei $\mathcal{A}^{\prime}$ eine $\mathcal{O}_{U}$-Ordnung in $A_{K}$, die $j^{*} \mathcal{B}$ enthält. Dann existiert eine $\mathcal{O}_{X}$-Ordnung $\mathcal{A}$ in $A_{K}$ mit $\mathcal{B} \subset \mathcal{A}$ und $j^{*} \mathcal{A}=\mathcal{A}^{\prime}$.

2. Ist $\mathcal{A}$ eine Maximalordnung, dann ist die Einschränkung von $\mathcal{A}$ auf jede offene Teilmenge maximal und für jeden Punkt $x \in X$ ist $A_{x}$ eine $\mathcal{O}_{X, x}$-Maximalordnung in $A_{K}$.

Bowne-Anderson, ein Schüler Chans, gibt die folgende Definition:

Definition ([BA11, Def. 2.1]). Sei $X$ ein normales, ganzes, noethersches Schema von endlichem Typ über einem algebraisch abgeschlossenen Körper $k$ mit char $k=0$. Eine Ordnung auf $X$ ist eine torsionsfreie kohärente $\mathcal{O}_{X}$-Algebra $A$, für die $K(A):=A \otimes_{\mathcal{O}_{X}} k(X)$ eine Azumaya-Algebra über dem Funktionenkörper $k(X)$ ist. 



\section{Literaturverzeichnis}

[AdJ] Artin, Michael und de Jong, Aise Johan: Stable orders over surfaces. www . math.1sa.umich. edu/courses/711/ordersms-num.pdf, work in progress.

[AG60a] Auslander, Maurice und Goldman, Oscar: The Brauer group of a commutative ring. Trans. Amer. Math. Soc., 97:367-409, 1960.

[AG60b] Auslander, Maurice und Goldman, Oscar: Maximal orders. Trans. Amer. Math. Soc., 97:1-24, 1960.

[AM69] Atiyah, Michael F. und Macdonald, Ian G.: Introduction to commutative algebra. Addison-Wesley Publishing Co., Reading, Mass. London - Don Mills, Ont., 1969.

[AVB90] Artin, Michael und Van den Bergh, Michel: Twisted homogeneous coordinate rings. J. Algebra, 133(2):249-271, 1990. http://dx.doi . org/10.1016/0021-8693(90)90269-T.

[Azu51] Azumaya, Gorô: On maximally central algebras. Nagoya Math. J., 2:119-150, 1951.

[BA11] Bowne-Anderson, Hugo: The Construction of Numerically CalabiYau Orders on Projective Surfaces. 2011. http://arxiv.org/abs/ 1107.0783, pre-print.

[Bae02] Baez, John C.: The octonions. Bull. Amer. Math. Soc. (N.S.), 39(2):145-205, 2002. http://dx.doi.org/10.1090/ S0273-0979-01-00934-X.

[Bix81] Bix, Robert: Isomorphism theorems for octonion planes over local rings. Trans. Amer. Math. Soc., 266(2):423-439, 1981. http://dx. doi.org/10.2307/1998432.

[Bou72] Bourbaki, Nicolas: Elements of mathematics. Commutative algebra. Hermann, Paris, 1972. Translated from the French. 
[Cha05] Chan, Daniel: Noncommutative cyclic covers and maximal orders on surfaces. Adv. Math., 198(2):654-683, 2005. http://dx.doi.org/ 10.1016/j.aim.2005.06.012.

[CI05] Chan, Daniel und Ingalls, Colin: The minimal model program for orders over surfaces. Invent. Math., 161(2):427-452, 2005. http: //dx.doi.org/10.1007/s00222-005-0438-z.

[CK03] Chan, Daniel und Kulkarni, Rajesh S.: del Pezzo orders on projective surfaces. Adv. Math., 173(1):144-177, 2003. http://dx.doi.org/ 10.1016/S0001-8708(02)00020-8.

[CK11] Chan, Daniel und Kulkarni, Rajesh S.: Moduli of bundles on exotic del Pezzo orders. Amer. J. Math., 133(1):273-293, 2011. http: //dx.doi.org/10.1353/ajm.2011.0002.

[dJ] de Jong, Aise Johan: A result of Gabber. www.math. columbia.edu/ $\sim$ dejong/papers/2-gabber.pdf, Preprint with missing references about $\mathrm{Br}=\mathrm{Br}^{\prime}$ on quasiprojective schemes.

[Fri98] Friedman, Robert: Algebraic surfaces and holomorphic vector bundles. Universitext. Springer-Verlag, New York, 1998.

[Gab81] Gabber, Ofer: Some theorems on Azumaya algebras. In: The Brauer group (Sem., Les Plans-sur-Bex, 1980), Band 844 der Reihe Lecture Notes in Mathematics, Seiten 129-209. Springer, Berlin, 1981.

[Gil12] Gille, Philippe: Octonion algebras over rings are not determined by their norms. 2012. http://arxiv.org/abs/1210.3947v2, pre-print.

[Gro61] Grothendieck, Alexander: Éléments de géométrie algébrique. II. Étude globale élémentaire de quelques classes de morphismes. Inst. Hautes Études Sci. Publ. Math., (8):222, 1961.

[Gro68a] Grothendieck, Alexander: Le groupe de Brauer. I. Algèbres d'Azumaya et interprétations diverses. In: Dix Exposés sur la Cohomologie des Schémas, Seiten 46-66. North-Holland, Amsterdam, 1968.

[Gro68b] Grothendieck, Alexander: Le groupe de Brauer. III. Exemples et compléments. In: Dix Exposés sur la Cohomologie des Schémas, Seiten 88-188. North-Holland, Amsterdam, 1968.

[GS06] Gille, Philippe und Szamuely, Tamás: Central simple algebras and Galois cohomology, Band 101 der Reihe Cambridge Studies in Advanced Mathematics. Cambridge University Press, Cambridge, 2006. http://dx.doi.org/10.1017/CB09780511607219. 
[GY03] Gan, Wee Teck und Yu, Jiu Kang: Schémas en groupes et immeubles des groupes exceptionnels sur un corps local. I. Le groupe $G_{2}$. Bull. Soc. Math. France, 131(3):307-358, 2003.

[Har77] Hartshorne, Robin: Algebraic geometry. Springer-Verlag, New York, 1977. Graduate Texts in Mathematics, No. 52.

[HL97] Huybrechts, Daniel und Lehn, Manfred: The geometry of moduli spaces of sheaves. Aspects of Mathematics, E31. Friedr. Vieweg \& Sohn, Braunschweig, 1997.

[HS05] Hoffmann, Norbert und Stuhler, Ulrich: Moduli schemes of generically simple Azumaya modules. Doc. Math., 10:369-389, 2005.

[Ker90] Kersten, Ina: Brauergruppen von Körpern. Aspects of Mathematics, D6. Friedr. Vieweg \& Sohn, Braunschweig, 1990.

[Ker07] Kersten, Ina: Brauergruppen. Universitätsdrucke Göttingen. Göttingen: Universitätsverlag, 2007.

[Kne02] Kneser, Martin: Quadratische Formen. Springer-Verlag, Berlin, 2002. Neu bearbeitet und herausgegeben in Zusammenarbeit mit Rudolf Scharlau.

[Knu91] Knus, Max Albert: Quadratic and Hermitian forms over rings, Band 294 der Reihe Grundlehren der Mathematischen Wissenschaften. Springer-Verlag, Berlin, 1991. With a foreword by I. Bertuccioni.

[KO74] Knus, Max Albert und Ojanguren, Manuel: Théorie de la descente et algèbres d'Azumaya, Band 389 der Reihe Lecture Notes in Mathematics. Springer-Verlag, Berlin, 1974.

[KO81] Knus, Max Albert und Ojanguren, Manuel: Cohomologie étale et groupe de Brauer. In: The Brauer group (Sem., Les Plans-sur-Bex, 1980), Band 844 der Reihe Lecture Notes in Mathematics, Seiten 210-228. Springer, Berlin, 1981.

[Lan02] Lang, Serge: Algebra, Band 211 der Reihe Graduate Texts in Mathematics. Springer-Verlag, New York, 3. Auflage, 2002.

[Lie07] Lieblich, Max: Moduli of twisted sheaves. Duke Math. J., 138(1):23-118, 2007. http://dx.doi.org/10.1215/ S0012-7094-07-13812-2.

[Mat86] Matsumura, Hideyuki: Commutative ring theory, Band 8 der Reihe Cambridge Studies in Advanced Mathematics. Cambridge University Press, Cambridge, 1986. Translated from the Japanese by M. Reid. 
[MF82] Mumford, David und Fogarty, John: Geometric invariant theory, Band 34 der Reihe Ergebnisse der Mathematik und ihrer Grenzgebiete. Springer-Verlag, Berlin, 2. Auflage, 1982.

[Mil80] Milne, James S.: Étale cohomology, Band 33 der Reihe Princeton Mathematical Series. Princeton University Press, Princeton, N.J., 1980.

[MO] Mumford, David und Oda, Tadao: Algebraic geometry II. http://hans.math.upenn.edu/ chai/624_08/math624_08.html, pre-print.

[Mum99] Mumford, David: The red book of varieties and schemes, Band 1358 der Reihe Lecture Notes in Mathematics. Springer-Verlag, Berlin, zweite, erweiterte Auflage, 1999. http://dx.doi.org/10.1007/ b62130.

[Neu92] Neukirch, Jürgen: Algebraische Zahlentheorie. Springer-Verlag, Berlin, 1992.

[Rei75] Reiner, Irving: Maximal orders. Academic Press [A subsidiary of Harcourt Brace Jovanovich, Publishers], London-New York, 1975. London Mathematical Society Monographs, No. 5.

[Rei03] Reiner, Irving: Maximal orders, Band 28 der Reihe London Mathematical Society Monographs. New Series. The Clarendon Press Oxford University Press, Oxford, 2003. Corrected reprint of the 1975 original, with a foreword by M. J. Taylor.

[Roe11] Roeseler, Karsten: Oktaven und Reduktionstheorie. Dissertation, Georg-August-Universität Göttingen, 2011. http://hdl.handle. net/11858/00-1735-0000-0006-B3F4-C.

[Sch66] Schafer, Richard D.: An introduction to nonassociative algebras. Pure and Applied Mathematics, Vol. 22. Academic Press, New York, 1966.

[Ser79] Serre, Jean Pierre: Local fields, Band 67 der Reihe Graduate Texts in Mathematics. Springer-Verlag, New York, 1979. Translated from the French by Marvin Jay Greenberg.

[Str02] Stroth, Kristin: Quaternionenalgebren über rationalen Funktionenkörpern in zwei Unbestimmten. Diplomarbeit, Georg-August-Universität Göttingen, 2002. http://num.math . uni-goettingen.de/ stroth/ mathe/diplom.pdf.

[Sus85] Suslin, Andrei A.: Algebraic K-theory and the norm residue homomorphism. Journal of Soviet Mathematics, 30:2556-2611, 1985. 
[SV00] Springer, Tonny A. und Veldkamp, Ferdinand D.: Octonions, Jordan algebras and exceptional groups. Springer Monographs in Mathematics. Springer-Verlag, Berlin, 2000.

[vBS59] van der Blij, Frederik und Springer, Tonny A.: The arithmetics of octaves and of the group $G_{2}$. Nederl. Akad. Wetensch. Proc. Ser. A $62=$ Indag. Math., 21:406-418, 1959. 



\title{
Symbolverzeichnis
}

\author{
$\mathbb{N}, \mathbb{N}_{0}$ \\ Menge der natürlichen Zahlen ohne bzw. mit 0 \\ $\mathbb{Z}, \mathbb{Q}, \mathbb{R}, \mathbb{C}$ \\ Menge der ganzen, rationalen, reellen, komplexen Zahlen \\ $\mathbb{H}$ \\ Hamiltonsche Quaternionenalgebra \\ $S^{\times}$ \\ $\mathbb{M}_{n}(S)$ \\ Einheitengruppe eines Ringes $S$ \\ $\operatorname{char}(K)$ \\ $\bar{K}$ \\ $K_{\mathrm{s}}$ \\ $K_{\mathrm{nr}}$ \\ $\hat{R}, \hat{K}$ \\ $\hat{M}$ \\ $A^{\text {op }}$ \\ $Z(A)$ \\ Ring der $n \times n$-Matrizen über einem Ring $S$ \\ Charakteristik eines Körpers $K$ \\ algebraischer Abschluss eines Körpers $K$ \\ separabler Abschluss von $K$ in $\bar{K}$ \\ maximal unverzweigte Erweiterung eines diskret bewerteten \\ Körpers $K$ in $K_{\mathrm{s}}$ \\ Vervollständigung eines diskreten Bewertungsringes oder \\ Körpers \\ $A \sim B$ \\ $[A]$ \\ zugehörige Skalarerweiterung $\hat{R} \otimes_{R} M$ eines $R$-Moduls $M$ \\ oppositionelle Algebra einer Algebra $A$ \\ Zentrum einer Algebra $A$ \\ $\operatorname{Br}(R), \operatorname{Br}(X)$ \\ Ähnlichkeit zweier Azumaya-Algebren $A$ und $B$ \\ $\operatorname{Br}_{n}(R), \operatorname{Br}_{n}(X)$ \\ Ähnlichkeitsklasse einer Azumaya-Algebra $A$ \\ $r_{R^{\prime} / R}$ \\ Brauergruppe eines Ringes $R$ oder Schemas $X$ \\ $\operatorname{Br}(L / K)$ \\ $\operatorname{Br}^{\prime}(X)$ \\ $n$-Torsionsuntergruppe der Brauergruppe \\ $(L, \operatorname{Gal}(L / K), f)$ \\ Restriktion $\operatorname{Br}(R) \rightarrow \operatorname{Br}\left(R^{\prime}\right)$ einer Ringerweiterung \\ relative Brauergruppe einer Körpererweiterung $L / K$ \\ kohomologische Brauergruppe eines Schemas $X$ \\ $(L, \sigma, a)$ \\ verschränktes Produkt zu einer Galoiserweit
und einem Kozykel $f \in \mathrm{H}^{n}\left(\operatorname{Gal}(L / K), L^{\times}\right)$ \\ zyklisch verschränktes Produkt zu einer zyklischen Erwei- \\ terung $L / K, \sigma \in \operatorname{Gal}(L / K)$ und $a \in K^{\times}$ \\ $(a, b, \zeta)$ \\ Normrestalgebra zu $a, b \in R^{\times}$und einer $n$-ten Einheitswur- \\ zel $\zeta$ \\ $\mathrm{H}^{n}(G, S) \quad n$-te Kohomologiegruppe einer Gruppe $G$ mit Werten in $S$ \\ $\mathcal{Z}^{n}(G, S) \quad$ Gruppe der $n$-Kozykel des Komplexes einer Standardauf- \\ lösung in der Gruppenkohomologie \\ $\mathrm{H}^{2}\left(X_{\text {et }}, \mathbb{G}_{\mathrm{m}}\right) \quad$ zweite Kohomologiegruppe in der étalen Kohomologie
}


Spec $R \quad$ affines Spektrum eines Ringes $R$

Proj $R \quad$ projektives Spektrum eines Ringes $R$

$\left(X, \mathcal{O}_{X}\right) \quad$ Schema $X$ mit Strukturgarbe $\mathcal{O}_{X}$

$k(x) \quad$ Restklassenkörper $\mathcal{O}_{X, x} / \mathfrak{m}_{x}$ in einem Punkt $x \in X$

$k(X) \quad$ Funktionenkörper eines ganzen Schemas $X$

$\eta \quad$ generischer Punkt eines ganzen Schemas

$X^{(1)} \quad$ Menge der Punkte von Kodimension 1 eines Schemas $X$

$\overline{\{x\}} \quad$ topologischer Abschluss eines Punktes $x \in X$

$\mathfrak{V}(x) \quad$ (abgeschlossene) Verschwindungsmenge

$D(f), D_{+}(f)$ offene Menge eines Polynoms $f$ in Spec $R$ oder Proj $R$

$\mathcal{A}, \mathcal{B}, \mathcal{C} \quad$ Garben, teilweise von Azumaya-Algebren, Maximalordnungen, Oktavenalgebren

$\mathcal{A}(x) \quad$ Quotient $\mathcal{A} \otimes k(x)$ im Punkt $x$

$\mathcal{L} \quad$ Geradenbündel, also ein lokalfreier $\mathcal{O}_{X}$-Modul von Rang 1

$\mathcal{L}^{\otimes n} \quad n$-faches Tensorprodukt von $\mathcal{L}$

$L_{\sigma}, \mathcal{L}_{\sigma} \quad$ mit $\sigma$ getwisteter Bimodul eines $R$-Moduls $L$ oder eines Geradenbündels $\mathcal{L}$

$\sigma^{*} L, \sigma^{*} \mathcal{L} \quad$ in der Linksmultiplikation getwisteter Modul

$\mathcal{O}_{X}(n) \quad$ getwistete Garbe

$\mathcal{L}(D) \quad$ Garbe zu einem Divisor $D$

$\operatorname{Spec} \mathcal{A} \quad$ Spektrum einer Garbe $\mathcal{A}$ von Algebren

$\mathrm{K}_{i}(F) \quad i$-te $K$-Gruppe eines Körpers $F$

$\{a, b\} \quad$ Element in der zweiten K-Gruppe

$r_{L / F} \quad$ Restriktion einer Körpererweiterung

$R_{n, \zeta} \quad$ Normresthomomorphismus

$\partial_{v}(\{a, b\}) \quad$ zahmes Symbol eines Elementes $\{a, b\}$

$\mathrm{N}_{L / K} \quad$ Norm einer Körpererweiterung

$(M, q) \quad$ quadratischer Modul, also ein Modul $M$ mit quadratischer Form $q$

$b(x, y),\langle x, y\rangle \quad$ Bilinearform auf den Elementen $x, y$ ausgewertet

$b_{M}$

Linearform $M \rightarrow M^{*}$ zu einer Bilinearform $b$

$M^{*}$

dualer Modul $\operatorname{Hom}_{R}(M, R)$ eines $R$-Moduls $M$

$d(M) \quad$ Diskriminante oder Diskriminantenideal eines quadratischen Moduls $M$

$(C, N) \quad$ Kompositionsalgebra $C$ mit Norm $N$ mit Einselement $e$

$\operatorname{Tr}(x) \quad$ Spur $\langle x, e\rangle$ eines Elementes $x \in C$

$\bar{x} \quad$ konjugiertes Element $\operatorname{Tr}(x) e-x$ zu $x$

$\{x, y, z\} \quad$ Assoziator $(x y) z-x(y z)$ dreier Elemente

$n(t) \quad$ Vielfaches einer Ähnlichkeit $t$ 
$(V, q) \quad$ Vektorraum mit quadratischer Form

$L^{\#} \quad$ duales Gitter eines Gitters $L$

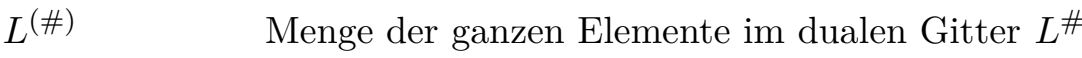

$O_{r}(L), O_{\ell}(L) \quad$ Rechts- bzw. Linksordnung eines Gitters $L$

$\operatorname{Ann}(M) \quad$ Annullator $\{r \in R \mid r m=0 \forall m \in M\}$ eines $R$-Moduls $M$

$\operatorname{Rad}(S) \quad$ Jacobson-Radikal eines Ringes $S$

$\operatorname{rg}_{\mathfrak{p}} M \quad$ Rang eines projektiven Moduls $M$ in einem Primideal $\mathfrak{p}$

$\delta_{i j} \quad$ Kroneckersymbol mit $\delta_{i i}=1$ und $\delta_{i j}=0$ für $i \neq j$

$e, f \quad$ Verzweigungsindex, Trägheitsgrad einer Fortsetzung einer diskreten Bewertung

Der Grundring einer Algebra ist stets ein kommutativer Ring mit Eins (und ist nicht der Nullring). 



\section{Index}

Symbole

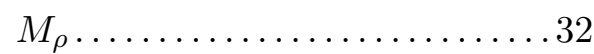

K-Gruppe.................22

$\rho^{*} M \ldots \ldots \ldots \ldots \ldots \ldots \ldots . \ldots . \ldots . \ldots 2$

\section{A}

ähnlich 2,5

Ähnlichkeit quadratischer Moduln.....665

Vielfaches...............6 65

Ähnlichkeitsklasse . . . . . . . . . . . . 2

Algebra................ 3, 121

einfach ................. 1

endlich-dimensional..........1

nicht assoziativ ...........52

oppositionelle ............. 2

separabel.................4

zentral .................. 1

alternative Algebra............60 60

anisotrop..................64

Assoziator....................60 60

ausgeartet..................54

Azumaya-Algebra........... 1, 3

B

Brauergruppe $.3,5$

C

Cayley-Algebra..............63

Cayley-Zahlen...............668

Charaktergruppe............. 19

\section{D}

Determinante .............. 53

Diskriminante...............55

Diskriminantenideal ........... 53

dualer Modul.................95

duales Gitter ............... 74

Dualraum.................. 95

E

einfach $\ldots \ldots \ldots \ldots \ldots \ldots$. 1, 122

endlich ................ 3, 121

endlich präsentiert............121

endlich-dimensional............ 1

\section{F}

flach ...................... 121

frei ....................... 121

freie Modulgarbe............ 136

\section{G}

ganz ................... 74

ganzes Gitter..............74

Schema ................ 135

ganzer Abschluss eines Schemas 43 Garbe

von Azumaya-Algebren.......3

gute Reduktion .......... 103

nichtassoziativer Algebren. 101

Ordnung.............. 137

schlechte Reduktion....... 103

von Oktavenalgebren...... 101

Gitter............... 15, 73 
dual................74

ganz ............... 74

maximal ............. 74

Rechts-, Linksordnung. . . . . .77

selbstdual ............74

Going-up, Going-down . . . . . . 127

Gruppe der Normenreste ..... . 12

gute Reduktion.............57

Garben .............. 103

\section{$\mathbf{H}$}

Henselsches Lemma . . . . . . . . . 128

\section{I}

idempotent.............. 126

inneres Produkt............ 55

Inverses

Kompositionsalgebra ...... 63

Involution . . . . . . . . . . . . . . . . . 59

Isometrie...............665

Isomorphie

von Kompositionsalgebren . . 66

isotrop . . . . . . . . . . . . . . . 64

$\mathbf{J}$

Jacobson-Radikal

\section{K}

K-Gruppe................22

kohärent ................... 135

kohomologische Brauergruppe ... 9

Komposition erlauben......55, 71

Kompositionsalgebra ......... 55

Isomorphie...........66 66

Quaternionenalgebra......63

über Ringen.............55

Kompositionsdivisionsalgebra . . 64

Konjugation.............. 59

Krullring ...........94, 133

\section{L}

Linksordnung

$.17,77$ lokal-noethersch.... . . . . . . . 135

lokalfreie Modulgarbe ........ 136

M

maximales Gitter........... 74

Maximalordnung ........... 16

Garbe (assoziativ) ...... 136

Garbe (nichtassoziativ)....103

nichtassoziativ .........76

Modul

regulär..............53

Monoid der Normenreste ..... . 36

multiplikative quad. Form . . . . . 55

\section{$\mathbf{N}$}

Nakayamas Lemma ... . . . . . . . 122

nichtausgeartet............ . 53

noethersch ............... . 135

Norm

Kompositionsalgebra ...... 55

normal................ 135

Normalisierung eines Schemas . . 43

Normrestalgebra ........... 13

Normresthomomorphismus . . . . 23

Nullstellendivisor . . . . . . . . . 26

Nullteiler

Kompositionsalgebra

\section{O}

Oktavenalgebra ............ 63

Oktonionenalgebra ..........6 63

oppositionelle Algebra ..........2

Ordnung ................ 16

Garbe (assoziativ) ....... 137

Garbe (nichtassoziativ)... 103

Maximalordnung ......... 16

nichtassoziativ ......... 76

Rechts-, Linksordnung. ... . .77

orthogonaler Untermodul......53

$\mathbf{P}$

Polstellendivisor .26 
primitiv .................. 81

projektiv .................. 122

\section{Q}

quadratische Algebra.........6 63

quadratische Form ........... 53

anisotrop...............64

isotrop ................6 64

multiplikativ .............55

nichtausgeartet...........53

regulär.................53

quadratischer Modul .......... 53

quasikohärent ................ 135

Quaternionenalgebra .......... 14

verallgemeinerte..........63

\section{$\mathbf{R}$}

Radikal ..................... 122

Rang

projektiver Modul........125

Rechtsordnung..........17, 77

reflexiv ....................95

regulär

quadratischer Modul ...... 53

Ring .................. 18

Schema .................. 19

relative Brauergruppe ......... 7

Restriktion ............ 6, 23

\section{S}

Satz

Going-up, Going-down . . . . 127

Henselsches Lemma ....... 128

Merkurjev-Suslin..........23

Nakayamas Lemma ....... 122

Skolem-Noether ............ 6

Wedderburn...............2

Schema

ganz................. 135

schlechte Reduktion

Garben ................ 103

selbstduales Gitter ............ 74 separabel.................... 4

Skolem-Noether (Satz) ....... 6

Spur

Kompositionsalgebra ...... 59

Standard-Involution ........... 59

Symbolalgebra ............... 13

$\mathbf{T}$

torsionsfrei ............... 136

Trägheitsgrad .............. 88

treu .................. 3, 121

treuflach ................. 122

treuprojektiv............... 122

\section{$\mathbf{U}$}

Überschneidungsbedingung . . . . . 40

Uniformisierende ............ . 89

Unterkompositionsalgebra ..... 60

unverzweigt ............... 21

V

verallgemeinerte

Quaternionenalgebra.....6 63

Verdopplung................61

Verdopplungsverfahren ........ 61

verschränktes Produkt..........7

zyklisches ............... 12

verzweigt .................. 21

Verzweigungsabbildung........ 20

Verzweigungsindex ........... 88

Vielfaches einer Ähnlichkeit . . . 65

volles Gitter..................15

vollständig ganzabgeschlossen . 133

W

Wedderburn (Struktursatz) ..... 2

Witt-Index ................ 64

$\mathbf{Z}$

zahmes Symbol..............24

Zariski-Brauergruppe..........9

zentral.............. $1,3,122$ 
zentral-einfache Algebra ........ 2

Zerfällungskörper............. 7

zerfallend .................. 64

zusammenhängender Ring. ....126

zyklisch verschränktes Produkt. 12

zyklische Algebra.............12 